\title{
Drift Compression and Final Focus Systems for Heavy Ion Inertial Fusion
}

\author{
by \\ Michiel Jan Laurens de Hoon \\ M. Eng. (Delft University of Technology) 1995 \\ A dissertation submitted in partial satisfaction of the \\ requirements for the degree of \\ Doctor of Philosophy \\ in \\ Physics \\ in the \\ GRADUATE DIVISION \\ of the \\ UNIVERSITY OF CALIFORNIA, BERKELEY
}

Committee in charge:

Professor Robert G. Littlejohn, Co-chair

Dr. Edward P. Lee, Co-chair

Professor Jonathan S. Wurtele

Professor Per F. Peterson

Spring 2001 
The dissertation of Michiel Jan Laurens de Hoon is approved:

\begin{tabular}{lr}
\hline Co-chair & Date \\
\hline Co-chair & Date \\
\hline & \\
\hline & Date \\
\hline
\end{tabular}

University of California, Berkeley

Spring 2001 
Drift Compression and Final Focus Systems for Heavy Ion

Inertial Fusion

Copyright 2001

by

Michiel Jan Laurens de Hoon 


\begin{abstract}
by

Michiel Jan Laurens de Hoon

Doctor of Philosophy in Physics

University of California, Berkeley

Professor Robert G. Littlejohn, Co-chair

Dr. Edward P. Lee, Co-chair
\end{abstract}

Drift Compression and Final Focus Systems for Heavy Ion Inertial Fusion

Longitudinal compression of space-charge dominated beams can be achieved by imposing a head-to-tail velocity tilt on the beam. This tilt has to be carefully tailored, such that it is removed by the longitudinal space-charge repulsion by the time the beam reaches the end of the drift compression section. The transverse focusing lattice should be designed such that all parts of the beam stay approximately matched, while the beam smoothly expands transversely to the larger beam radius needed in the final focus system following drift compression. In this thesis, several drift compression systems were designed within these constraints, based on a given desired pulse shape at the end of drift compression. The occurrence of mismatches due to a rapidly increasing current was analyzed. In addition, the sensitivity of drift compression to errors in the initial velocity tilt and current profile was studied. These calculations were done using a new computer code that accurately calculates the longitudinal electric field in the space-charge dominated regime. 
In final focus, the beam is usually expanded transversely before it is focused onto a small spot. This may cause third-order aberrations to significantly affect the beam quality. Previously, it has been suggested that octupoles be used to correct these aberrations. However, it is shown that for modest convergence angles of the beam, a considerable improvement can be achieved by rematching the quadrupoles in the final focus system, if these aberrations are taken into account in the rematching process. For larger convergence angles, third-order aberrations cause a significant halo formation.

Detailed particle-in-cell simulations were performed to better understand the experimental results from the Scaled Final Focus Experiment at LBNL, which is a replica of a driver-scale final focus system. The simulations showed that expanding beams may suffer a substantial emittance increase due to their nonlinear space-charge field. Phasespace comparisons between the experiment and simulations showed reasonable agreement. Of particular interest was the beam rotation that has been measured in the experiment. Simulations showed that this may have been caused by a minor rotation of one or more of the quadrupoles in the experiment.

Professor Robert G. Littlejohn Dissertation Committee Co-chair

Dr. Edward P. Lee

Dissertation Committee Co-chair 


\section{Contents}

List of Figures $\quad$ v

List of Tables $\quad$ xi

1 Introduction 1

1.1 The Role of Fusion in Meeting the Global Energy Demand . . . . . . . . . . 2

1.2 Principles of Fusion . . . . . . . . . . . . . . . . . 4

1.3 Heavy Ion Inertial Fusion . . . . . . . . . . . . . . . . . . . 13

1.4 Overview of Conceptual Heavy Ion Drivers and Experiments . . . . . . . 16

1.4 .1 HIBALL-II Design . . . . . . . . . . . . . . . . 17

1.4 .2 HYLIFE-II Design . . . . . . . . . . . . . . . . . . 18

1.4.3 Small and Intermediate Scale Experiments . . . . . . . . . . . . . . 19

1.5 Purpose of Thesis . . . . . . . . . . . . . . . . . . 23

2 Charged Particle Beam Dynamics 29

2.1 Beam Dynamics in the Zero-Current Limit . . . . . . . . . . . . . . . 29

2.2 Beam Dynamics in the Presence of Space Charge . . . . . . . . . . . . 33

2.3 Beam Dynamics in the Smooth Approximation . . . . . . . . . . . . . 39

2.3.1 Approximate Envelope Relations . . . . . . . . . . . . . . . . 41

2.4 Nonlinear Effects . . . . . . . . . . . . . . . . . . . . 41

2.4.1 Third Order Aberrations . . . . . . . . . . . . . . . . . . 42

2.4.2 Chromatic Aberrations . . . . . . . . . . . . 46

2.4 .3 Image Charges . . . . . . . . . . . . . . . . . . . . . . . . 47

2.4.4 Higher Multipole Fields . . . . . . . . . . . . . . . . . . . . 47

2.4 .5 Misalignments . . . . . . . . . . . . . . . . . 48

2.4.6 Beam Distribution . . . . . . . . . . . . . . . . . 48

2.4.7 Three-Dimensional Effects . . . . . . . . . . . . . . . . . . . 49

2.4.8 Presence of Background Gas and Electrons . . . . . . . . . . . . 49

3 Simulation Codes $\quad 51$

3.1 JavaEnvelope . . . . . . . . . . . . . . . . . . . . . . . . . 52

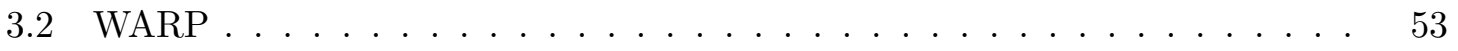


$3.2 .1 \quad$ Envelope Solver . . . . . . . . . . . . . . . . . . . . 54

3.2.2 WARP3d, WARPxy, and WARPrz . . . . . . . . . 54

3.2 .3 The CIRCE Module . . . . . . . . . . . . . . . . . . . . 59

3.2 .4 The Hermes Project . . . . . . . . . . . . . . . . . . . 63

4 The IRE Drift Compression Section $\quad \mathbf{8 4}$

4.1 Designing a Drift Compression Section . . . . . . . . . . . . . . . 85

4.2 IRE Drift Compression Designs . . . . . . . . . . . . . . . . . . . 96

4.3 Sensitivity Studies . . . . . . . . . . . . . . . . . . . . 103

4.3.1 Systematic Velocity Errors . . . . . . . . . . . . . . . 103

4.3.2 Random Current Errors . . . . . . . . . . . . . . . . . . . . . . . . 104

4.3.3 Systematic Current Errors . . . . . . . . . . . . . . . . . . 108

5 The IRE Final Focus System 110

5.1 Design of a Final Focus System . . . . . . . . . . . . . . . . . . . . . 111

5.2 Third-Order Aberrations . . . . . . . . . . . . . . . . . . . . . 112

5.3 PIC Code Rematching . . . . . . . . . . . . . . . . . . . . . . . 119

6 The Scaled Final Focus Experiment 130

6.1 The Scaled HIBALL-II Case . . . . . . . . . . . . . . . . . . . . . . . . 134

6.1.1 Emittance Growth of Expanding Charged Particle Beams . . . . . . 142

$6.1 .2 \quad$ Fringe Field Effects . . . . . . . . . . . . . . . . . . 150

6.2 The Modified Scaled HIBALL-II Case . . . . . . . . . . . . . . . . . 158

6.3 The High Scaled Current Case . . . . . . . . . . . . . . . . . . . . . 162

6.4 Beam Rotation . . . . . . . . . . . . . . . . . . . . . . . 181

$\begin{array}{llr}7 & \text { Summary } & 214\end{array}$

$\begin{array}{lr}\text { Bibliography } & 217\end{array}$

A Python Scripts Used $\quad 232$

A.1 Drift Compression Design . . . . . . . . . . . . . . . . . . . 232

A.2 PIC Code Rematching of Final Focus Systems . . . . . . . . . . . . . 243 


\section{List of Figures}

1.1 The estimated cost of electricity for several conceptual fusion power plant designs in comparison to natural gas, coal, and fission. . . . . . . . . .

1.2 Reaction rate parameters for Maxwellian distributions as a function of tem-

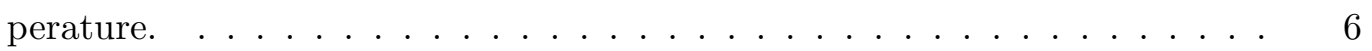

1.3 The DIII-D Tokamak at General Atomics. . . . . . . . . . . . . 8

1.4 Energy flows in a power plant based on inertial fusion. . . . . . . . . . . . 10

1.5 A hohlraum for heavy-ion driven IFE, showing a DT fuel pellet inside a cylindrical casing lined with metal. . . . . . . . . . . . . . . . 11

1.6 Conceptual diagram of a heavy-ion linear accelerator for IFE. . . . . . . . 16

1.7 The HYLIFE-II chamber. . . . . . . . . . . . . . . . . . . . . . . . 19

1.8 The Scaled Final Focus Experiment. . . . . . . . . . . . . . 20

2.1 The quadrupole field gradient in the fringe field of a quadrupole . . . . . 45

3.1 Schematic of the leap-frog algorithm. . . . . . . . . . . . 56

3.2 Schematic of the CIRCE model. . . . . . . . . . . . . . . . 61

3.3 Schematic of the Hermes model. . . . . . . . . . . . . . . . . . 66

3.4 The volume weighting scheme used in Hermes for charge deposition. . . . . 67

3.5 Scaling factor for the charge deposition in the Hermes model, as determined by the relative position of the slice boundaries to the grid points. . . . . . . 68

3.6 Interpolation scheme for the longitudinal electric field in Hermes. . . . . . . 69

3.7 The beam current profile for an initially $100 \mathrm{~ns}$ beam of $46.875 \mathrm{~A}$, as calculated by the Hermes model and the $g$-factor model. . . . . . . . . . . . .

3.8 The beam current profile for an initially $5 \mathrm{~ns}$ beam of $937.5 \mathrm{~A}$ after traveling various distances along the transport lattice, as calculated by the Hermes model and by the $g$-factor model. . . . . . . . . . . . . .

3.9 The beam current profile for an initially $5 \mathrm{~ns}$ beam of 937.5 A after traveling four lattice half periods, as calculated by the Hermes model. . . . . . . . . .

3.10 The beam current profile for an initially $5 \mathrm{~ns}$ beam of $937.5 \mathrm{~A}$ after traveling ten lattice half periods, as calculated by WARP3d, by Hermes, and by the $g$-factor model. . . . . . . . . . . . . . . . . 
3.11 The repulsive electric field at the surface of a slice, compressed to zero length, as a function of the beam radius of the slice. . . . . . . . . . . .

4.1 The current profile as a function of position, for a $20 \mathrm{~ns}$ final pulse duration, if the beam is not rematched at the beginning of drift compression. . . . . .

4.2 The transverse beam envelope for the beam center, for a $20 \mathrm{~ns}$ final pulse duration, if the beam is not rematched at the beginning of drift compression. 91

4.3 The current for different slice boundaries of the beam for a $20 \mathrm{~ns}$ final pulse duration, if the beam is not rematched at the beginning of drift compression. 92

4.4 The transverse beam envelope for the second slice boundary of the beam, for a 20 ns final pulse duration, if the beam is not rematched at the beginning of drift compression. . . . . . . . . . . . . . . . . . . .

4.5 The transverse beam envelope for the second slice boundary of the beam, for a $20 \mathrm{~ns}$ final pulse duration, if the beam is rematched at the beginning of drift compression. . . . . . . . . . . . . . . .

4.6 The current profile as a function of position, for a $20 \mathrm{~ns}$ final pulse duration, if the beam is rematched at the beginning of drift compression. . . . . . . .

4.7 The current profile as a function of position, at the end of drift compression, for different final pulse durations, if the beam is rematched at the beginning of drift compression. . . . . . . . . . . . . . . .

4.8 The initial velocity tilt with respect to the beam center, for $20 \mathrm{~ns}, 15 \mathrm{~ns}$, and 10 ns final pulse durations. . . . . . . . . . . . . . .

4.9 The current profile as a function of position, at various locations along the drift compression section, for a 10 ns final pulse duration. . . . . . . . . .

4.10 The current profile at the end of drift compression, as a function of position, for the case of a $10 \mathrm{~ns}$ final pulse duration if the initial head-to-tail velocity tilt is $1 \%$ too low. . . . . . . . . . . . . . . . . .

4.11 An example of the current profile at the end of drift compression, as a function of position, for a $10 \mathrm{~ns}$ final pulse duration, if the initial current has a $1 \%$ random error. . . . . . . . . . . . . . . . .

4.12 The current profile at the end of drift compression, as a function of position, for a $10 \mathrm{~ns}$ final pulse duration, if the initial current is too low by $1 \%$. . . .

5.1 The focusing solutions for different values of the convergence angle, as calculated from the envelope equations. . . . . . . . . . . . . . . 113

5.2 Continuation of figure $5.1 \ldots \ldots \ldots \ldots \ldots \ldots \ldots$

5.3 The magnetic field gradients of the four quadrupoles as a function of the convergence angle, as calculated from the envelope equations. . . . . . . . .

5.4 The focal spot radius as a function of the convergence angle, as calculated from the envelope equations. . . . . . . . . . . . 116

5.5 The focal spot radius as a function of the convergence angle, as calculated from the envelope equations, and from WARPxy simulations, taking into account third-order and space-charge aberrations. . . . . . . . . . . 
5.6 The horizontal and vertical beam radius as calculated from the WARPxy simulations, for different values of the convergence angle, using the magnetic field gradients that were found from the envelope equations. . . . . . . . .

5.7 Continuation of figure $5.6 \ldots \ldots \ldots \ldots \ldots$

5.8 The focal spot radius as a function of the convergence angle, as calculated from the envelope equations, and from WARPxy simulations, after rematching the magnetic field gradients of the quadrupoles using WARPxy. . . . . .

5.9 The magnetic field gradients of the four quadrupoles as a function of the convergence angle, after rematching using WARPxy. . . . . . . . . . .

5.10 The horizontal and vertical beam radius as calculated from the WARPxy simulations, for convergence angles between 10 and $16 \mathrm{mrad}$, after rematching the magnetic field gradients of the quadrupoles using the WARPxy code. .

5.11 The horizontal and vertical beam radius as calculated from the WARPxy simulations, for a $17 \mathrm{mrad}$ convergence angle, after rematching the magnetic field gradients of the quadrupoles using the WARPxy code. . . . . . . . . .

5.12 The particle density in configuration space at the target location, for different values of the convergence angle, after rematching the magnetic field gradients of the quadrupoles using the WARPxy code. . . . . . . . . . . . . . . .

5.13 Continuation of figure $5.12 \ldots \ldots \ldots \ldots \ldots$

6.1 The conducting boundary of an electrostatic quadrupole in the Scaled Final Focus Experiment. . . . . . . . . . . . . . . . .

6.2 The Scaled Final Focus Experiment. The beam envelope shown corresponds to the scaled HIBALL-II case. . . . . . . . . . . . . . . . .

6.3 The horizontal and vertical beam envelope, extent of the grid, and the location of the conducting boundaries in the simulation of the scaled HIBALL-II case. . . . . . . . . . . . . . . . . . . .

6.4 The horizontal and vertical beam radius of an initially semi-Gaussian beam as a function of the longitudinal distance traveled, for the scaled HIBALL-II case. . . . . . . . . . . . . . . . . . .

6.5 The edge emittance of an initially semi-Gaussian beam as a function of the longitudinal distance traveled, for the scaled HIBALL-II case. . . . . . . . .

6.6 The edge emittance of a $\mathrm{K}-\mathrm{V}$ beam as a function of the longitudinal distance traveled, for the scaled HIBALL-II case. . . . . . . . . . . . . . .

6.7 Sheared $x-x^{\prime}$ phase space at the end of the expansion drift section, just before the first magnetic quadrupole, for the scaled HIBALL-II case. . . . .

6.8 Sheared $y-y^{\prime}$ phase space at the end of the expansion drift section, just before the first magnetic quadrupole, for the scaled HIBALL-II case. . . . .

6.9 Relative emittance increase in WARPxy particle-in-cell simulations of expanding beams . . . . . . . . . . . . . . . . . 148

6.10 The slope of the linear part of the emittance increase in figure 6.9. . . . . 149

6.11 Sheared phase space of an expanding beam. . . . . . . . . . . . . 151

6.12 The edge emittance of an initially semi-Gaussian beam as a function of the longitudinal distance traveled, if fringe fields are included, for the scaled HIBALL-II case. . . . . . . . . . . . . . . . . . . . 153 
6.13 The edge emittance of an initially $\mathrm{K}-\mathrm{V}$ beam as a function of the longitudinal distance traveled, if fringe fields are included, for the scaled HIBALL-II case. 154

6.14 The edge emittance of an initially semi-Gaussian beam as a function of the longitudinal distance traveled, for the scaled HIBALL-II case. This simulation included only the longitudinal field $e / b_{2,0}^{\prime}(z)$ caused by the fringe fields of the electrostatic/magnetic quadrupoles. . . . . . . . . . . . . .

6.15 The magnitude of the quadrupole and the pseudo-octupole component of the fringe field at the end of the third magnet as a function of position, together with the edge emittance of an initially semi-Gaussian beam. . . . . . . . .

6.16 The Scaled Final Focus Experiment. The beam envelope shown corresponds to the modified scaled HIBALL-II case. . . . . . . . . . . . . .

6.17 The horizontal and vertical beam envelope, extent of the grid, and the location of the conducting boundaries in the simulation of the modified scaled HIBALL-II case. . . . . . . . . . . . . . . . . . .

6.18 The edge emittance of an initially semi-Gaussian beam as a function of the longitudinal distance traveled, as well as the emittance measurements from the experiment, for the modified scaled HIBALL-II case. . . . . . . . . . .

6.19 The horizontal and vertical beam radius of an initially semi-Gaussian beam as a function of the longitudinal distance traveled, for the modified scaled HIBALL-II case. Both the results from the baseline particle-in-cell simulation and an envelope calculation are shown. . . . . . . . . . . .

6.20 The Scaled Final Focus Experiment. The beam envelope shown corresponds to the higher scaled current case. . . . . . . . . . . . . .

6.21 The horizontal and vertical beam radius of an initially semi-Gaussian beam as a function of the longitudinal distance traveled for the higher scaled current case. . . . . . . . . . . . . . . . . .

6.22 The horizontal and vertical beam envelope, extent of the grid, and the location of the conducting boundaries in the simulation of the higher scaled current case. . . . . . . . . . . . . . . . . .

6.23 The edge emittance of an initially semi-Gaussian beam as a function of the longitudinal distance traveled, as well as the emittance measurements from the experiment, for the higher scaled current case. . . . . . . . . . . .

6.24 The edge emittance of an initially $\mathrm{K}-\mathrm{V}$ beam as a function of the longitudinal distance traveled, for the higher scaled current case. . . . . . . . . . . . . 172

6.25 Sheared vertical phase space at diagnostic station D1. . . . . . . . . . . . 174

6.26 Sheared horizontal phase space at diagnostic station D2 . . . . . . . . 175

6.27 Sheared vertical phase space at diagnostic station D2. . . . . . . . . 176

6.28 Sheared horizontal phase space at diagnostic station D3. . . . . . . . . 177

6.29 Sheared vertical phase space at diagnostic station D3. . . . . . . . . . . 178

6.30 Sheared horizontal phase space at diagnostic station D4 . . . . . . . . . 179

6.31 Sheared vertical phase space at diagnostic station D4. . . . . . . . . . . . 180

6.32 The horizontal and vertical beam radius of a $\mathrm{K}-\mathrm{V}$ beam as a function of the longitudinal distance traveled, in the presence of a quadrupole rotation. . . 
6.33 The beam orientation, calculated from the moment equations, at several locations along the lattice, in the presence of a $1.24^{\circ}$ rotation of the second magnetic quadrupole. . . . . . . . . . . . . . . . . . . 188

6.34 Continuation of figure $6.33 . \ldots \ldots \ldots \ldots$

6.35 The emittance of a $\mathrm{K}-\mathrm{V}$ and a semi-Gaussian beam as a function of the longitudinal distance traveled, calculated from the moment equations and from a 2D transverse slice particle-in-cell simulation using WARP, in the presence of a quadrupole rotation. . . . . . . . . . . . . .

6.36 The generalized emittance of a $\mathrm{K}-\mathrm{V}$ and a semi-Gaussian beam as a function of the longitudinal distance traveled, calculated from a $2 \mathrm{D}$ transverse slice particle-in-cell simulation using WARP, in the presence of a quadrupole rotation. . . . . . . . . . . . . . . .

6.37 Current distribution of the beam as measured in the experiment at diagnostic location $\mathrm{D} 4$, using the focusing lattice given in table $6.13 . \ldots . . . .$.

6.38 Current distribution of the beam as measured in the experiment at diagnostic location D4, together with the beam ellipse as calculated from the moment equations assuming that the second quadrupole is rotated. . . . . . . . .

6.39 Current distribution of the beam as measured in the experiment at diagnostic location D4, together with the beam ellipse as calculated from the moment equations assuming that the third quadrupole is rotated. . . . . . . . .

6.40 Current distribution of the beam as measured in the experiment at diagnostic location D4, together with the beam ellipse as calculated from the moment equations assuming that the first, second, and third quadrupole are rotated.

6.41 Current distribution of the beam as measured in the experiment at diagnostic location D4, together with the beam ellipse as calculated from the moment equations assuming that all quadrupoles are rotated. . . . . . . . . .

6.42 Current distribution of the beam as measured in the experiment at diagnostic location D4, using the focusing lattice given in table 6.14. . . . . . . . .

6.43 Current distribution of the beam as measured in the experiment at diagnostic location D4, together with the beam ellipse as calculated from the moment equations assuming that the second quadrupole is rotated. . . . . . . . .

6.44 Current distribution of the beam as measured in the experiment at diagnostic location D4, together with the beam ellipse as calculated from the moment equations assuming that the first, second, and third quadrupole are rotated.

6.45 Current distribution of the beam as measured in the experiment at diagnostic location D4, together with the beam ellipse as calculated from the moment equations assuming that all six quadrupoles are rotated. . . . . . . . .

6.46 Current distribution of the beam as measured in the experiment at diagnostic station D4, using the focusing lattice given in table $6.15 \ldots \ldots \ldots$

6.47 Current distribution of the beam as measured in the experiment at diagnostic station D4, using the focusing lattice given in table 6.15 but with a magnetic field gradient of $1.83 \mathrm{~T} / \mathrm{m}$ for the fifth magnetic quadrupole. . . . . . . . 
6.48 Current distribution of the beam as measured in the experiment at diagnostic location D4, together with the beam ellipse as calculated from the moment equations assuming that the first, second, and third quadrupole are rotated. 207

6.49 Current distribution of the beam as measured in the experiment at diagnostic location D4, together with the beam ellipse as calculated from the moment equations assuming that all six quadrupoles are rotated. . . . . . . . . 208

6.50 Current distribution of the beam as measured in the experiment at diagnostic location D4, using the focusing lattice given in table 6.17. . . . . . . . . . 210

6.51 Current distribution of the beam as measured in the experiment at diagnostic location D4, together with the beam ellipse as calculated from the moment equations assuming that the second quadrupole is rotated. . . . . . . . .

6.52 Current distribution of the beam as measured in the experiment at diagnostic location D4, together with the beam ellipse as calculated from the moment equations assuming that all six quadrupoles are rotated. . . . . . . . . . . . 212 


\section{List of Tables}

1.1 Plant parameters of the HYBALL-II and HYLIFE-II conceptual power plant designs. . . . . . . . . . . . . . . . .

1.2 Beam parameters for the preliminary IRE design, and for a typical power plant based on Heavy Ion Inertial Fusion. . . . . . . . . . . . . . . . .

1.3 Beam parameters of the preliminary IRE design at different locations along the accelerator. . . . . . . . . . . . . . . . . . .

3.1 Beam parameters of the $100 \mathrm{~ns}$ test beam in Hermes. . . . . . . . . . . 70

3.2 The transport lattice used for the $100 \mathrm{~ns}$ test beam in Hermes. . . . . . . . 70

3.3 Beam parameters of the 5 ns test beam in Hermes. . . . . . . . . . . . . 72

3.4 The transport lattice used for the 5 ns test beam in Hermes. . . . . . . . . 73

3.5 The current at the beam center after traveling ten lattice half periods, for the 5 ns test beam, as calculated by Hermes using different numerical parameters. 75

3.6 The current at the beam center after traveling ten lattice half periods, for the 5 ns test beam, as calculated by WARP3d using different numerical parameters. 76

4.1 Numerical parameters used in the Hermes calculations for the IRE drift compression designs. . . . . . . . . . . . . . . . .

4.2 Initial velocity tilt, drift compression length, and deviation from the initially specified beam current at the end of drift compression due to rematching at the beginning of drift compression, as calculated by Hermes. . . . . . . . . .

4.3 RMS deviation from the initially specified beam current at the end of drift compression, if the initial head-to-tail velocity tilt is $1 \%$ too low. . . . . . .

4.4 RMS deviation from the initially specified beam current at the end of drift compression, due to 1\% RMS random errors in the initial current. . . . . . 106

4.5 RMS deviation from the initially specified beam current multiplied by 0.99 , at the end of drift compression, if the initial current is too low by $1 \%$. . . 108

5.1 Simulation parameters for the IRE final focus simulations. . . . . . . . . 117

6.1 Beam parameters for the scaled HIBALL-II case. . . . . . . . . . . . . . 136

6.2 Lattice properties for the scaled HIBALL-II case. . . . . . . . . . . . . 136

6.3 Simulation results for the scaled HIBALL-II case. . . . . . . . . . . . . 138 
6.4 Beam parameters for the modified scaled HIBALL-II case. . . . . . . . . . . 159

6.5 Lattice properties for the modified scaled HIBALL-II case. . . . . . . . . . . 160

6.6 Simulation results for the modified scaled HIBALL-II case. . . . . . . . . . 160

6.7 Beam parameters for the higher scaled current case. . . . . . . . . . . . 165

6.8 Lattice properties for the higher scaled current case. . . . . . . . . . . . . 167

6.9 Simulation results for the higher scaled current case. . . . . . . . . . . . . 171

6.10 Emittance measured in the experiment at the second and fourth diagnostic station in the scaled HIBALL-II case. . . . . . . . . . . . . . . . . . 184

6.11 Several combinations of rotation angles for the magnetic quadrupoles that give rise to a sixfold increase in the horizontal and vertical emittance. . . . 185

6.12 Beam parameters for the first five beam rotation experiments. . . . . . . . . 187

6.13 Lattice properties for the second beam rotation experiment. . . . . . . . . . 192

6.14 Lattice properties for the third beam rotation experiment. . . . . . . . . . . 194

6.15 Lattice properties for the fourth beam rotation experiment. . . . . . . . . . 200

6.16 Beam parameters for the beam rotation experiment using a smaller initial beam. . . . . . . . . . . . . . . . . 205

6.17 Lattice properties for the beam rotation experiment using the smaller initial

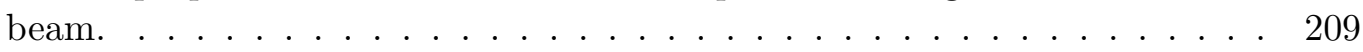




\section{Acknowledgements}

The work presented in this dissertation has benefited tremendously from numerous people, whose support and guidance I am most thankful for. I would like to express my gratitude to some of the people who particularly contributed to this undertaking, either as a teacher, a colleague, or a friend.

First, I would like to acknowledge the late Dr. David Judd, who was my initial faculty advisor in the Department of Physics. His unbounded passion for physics in general and heavy ion fusion in particular has been and still is an inspiration to many of us, especially young scientists.

The guidance and support of my research advisor, Dr. Edward Lee, has been instrumental throughout my years in graduate school with regard to both scientific as well as practical issues. Particularly impressive is his willingness to answer the most basic questions, together with his ability to answer the most difficult questions.

I would like to thank Professor Robert Littlejohn for continuing the work of Dr. David Judd as my faculty advisor, and to Professors Jonathan Wurtele and Per Peterson for serving on my dissertation committee. Professor Per Peterson provided the very useful background on engineering aspects of heavy ion inertial fusion. I am also indebted to Professors Ken Fowler and Allan Kaufman, who were my first teachers of fusion research and plasma physics at the University of California, Berkeley.

Dr. David Grote deserves my special gratitude for his willingness to answer my many questions on the WARP simulation code. Without Dave, most of the results presented here would have been impossible to obtain. I would like to express my appreciation to 
Dr. John Barnard for our enlightening discussions on many of the topics contained in this thesis, particularly with respect to longitudinal dynamics and skew quadrupoles. I would also like to thank Dr. Alex Friedman for his encouragement and support in the development of novel numerical methods.

I would also like to thank the staff of the Fusion Energy Research Group at LBNL, Ms. Lynn Heimbucher, Ms. Sharon Buckley, and Mr. Paijoun MontannaBronte, together with Ms. Anne Takizawa, the student affairs officer of the Department of Physics.

Special thanks are due to Dr. Scott Armel, Dr. Jérôme Verbeke, Dr. Steve MacLaren, Mr. Dave Ponce, Mr. Lionel Prost, and Mr. Edwin Chacon-Golcher for their loyal attendance at the fusion student seminar at $3 \mathrm{pm}$ in building 54 . It was at this seminar where the major conceptual breakthroughs in heavy ion fusion were achieved. I would like to thank my office mate Dr. Steve MacLaren also for explaining to me the details of the Scaled Final Focus Experiment, his willingness to clarify repeatedly which file contains which data, and for helping me during my brief days of homelessness. With my office mate Dr. Caron Jantzen, I shared many hours analyzing core dump files when we were struggling with our simulation codes. I would like to thank Mr. Lionel Prost especially for his help with the beam rotation experiments.

At the International House, Berkeley, I found my new friends, who made my stay in California most enjoyable. Soon we will meet again in each other's home countries. Last but not least, I would like to thank my family for their support throughout my life on Cyclotron Road. 


\section{Chapter 1}

\section{Introduction}

The past fifty years have been an age of rapid economic growth. Global prosperity increased at a rate unparalleled in human history. Although some countries enjoyed a larger share of the new wealth than others, the trend of rapid economic growth is world-

wide. It was made possible by advances in science and technology, expressed in the form of industrialization and improved efficiency [85].

The greater prosperity around the world enabled it to sustain a larger population. The world population has more than tripled in the last century, and is predicted to grow by nearly 80 million people annually during the next 25 years. Most of the population growth will occur in developing countries [99].

The combination of the rapid economic growth and an increasing world population resulted in an ongoing depletion of the global fossil fuel reserves. Additionally, the increased emissions of carbon dioxide gas lead to the greenhouse effect, causing a global temperature increase. The subsequent melting of ice in the polar regions causes the sea level to rise, 
endangering low-lying countries such as Bangladesh and the Netherlands. Furthermore, the dumping of melted ice into the saltwater of the surrounding oceans may disrupt ocean flows, which would severely change climate conditions around the world [57].

The increasing awareness of these environmental issues as well as concern over depletion of the limited global reserves of fossil fuel, which are furthermore located in a relatively small number of countries, spurred a search for alternative energy sources. Besides renewable energy sources and nuclear fission, nuclear fusion, if proven feasible and economical, can contribute significantly to the long-term global energy need. While nuclear fission provides a substantial part of the energy need in developed countries, its desirability is limited due to the possibility of nuclear accidents, the generation of long-term radioactive waste, and proliferation [53].

\subsection{The Role of Fusion in Meeting the Global Energy De- mand}

In order to keep up with the rising global demand for energy, new sources of energy have to be developed. Fusion energy is attractive for several reasons.

The fuel needed for fusion energy consists of lithium and deuterium. Vast reserves are widely available of both of these. Fusion blankets use and consume additional materials, such as beryllium, depending on design. However, again supplies are abundant compared to the quantities needed. Neither lithium nor deuterium are toxic or radioactive.

While burning fossil fuel produces greenhouse gases that cause global warming, the emissions due to fusion energy are practically zero. In addition, no significant release 
of tritium during an accident is conceivable, if the plant is carefully designed.

Fusion does not involve any highly radioactive reaction products such as those generated in fission reactions. The radioactive waste created by fusion is therefore less hazardous than the waste created by fission.

Fusion reactors are inherently safe, because they contain only a small amount of fuel at any given time. Furthermore fusion is not based on a chain reaction as in case of nuclear fission. The occurrence of large-scale accidents with grave consequences for the general public is therefore very unlikely.

Whether fusion will be attractive as an energy source ultimately depends on its cost of electricity in comparison to other energy sources. Because of the environmental advantages of fusion, it does not need to be the cheapest source of energy; an affordable cost of electricity will suffice. Figure 1.1 shows the estimated cost of electricity for several conceptual fusion power plant designs, as well as for natural gas, coal and nuclear fission [63]. Although natural gas may remain the cheapest source of energy in the near future, particularly heavy ion fusion is expected to be able to provide electricity at a reasonable cost. Note that the price of natural gas may escalate in the decades to come due to depletion.

Research and development in fusion energy may provide spin-offs in technology areas such as superconducting magnets, high-power lasers, and supercomputing [53]. Commercial fusion power plants may be built by the middle of the 21st century, gradually replacing fossil fuel and nuclear fission plants [53]. 


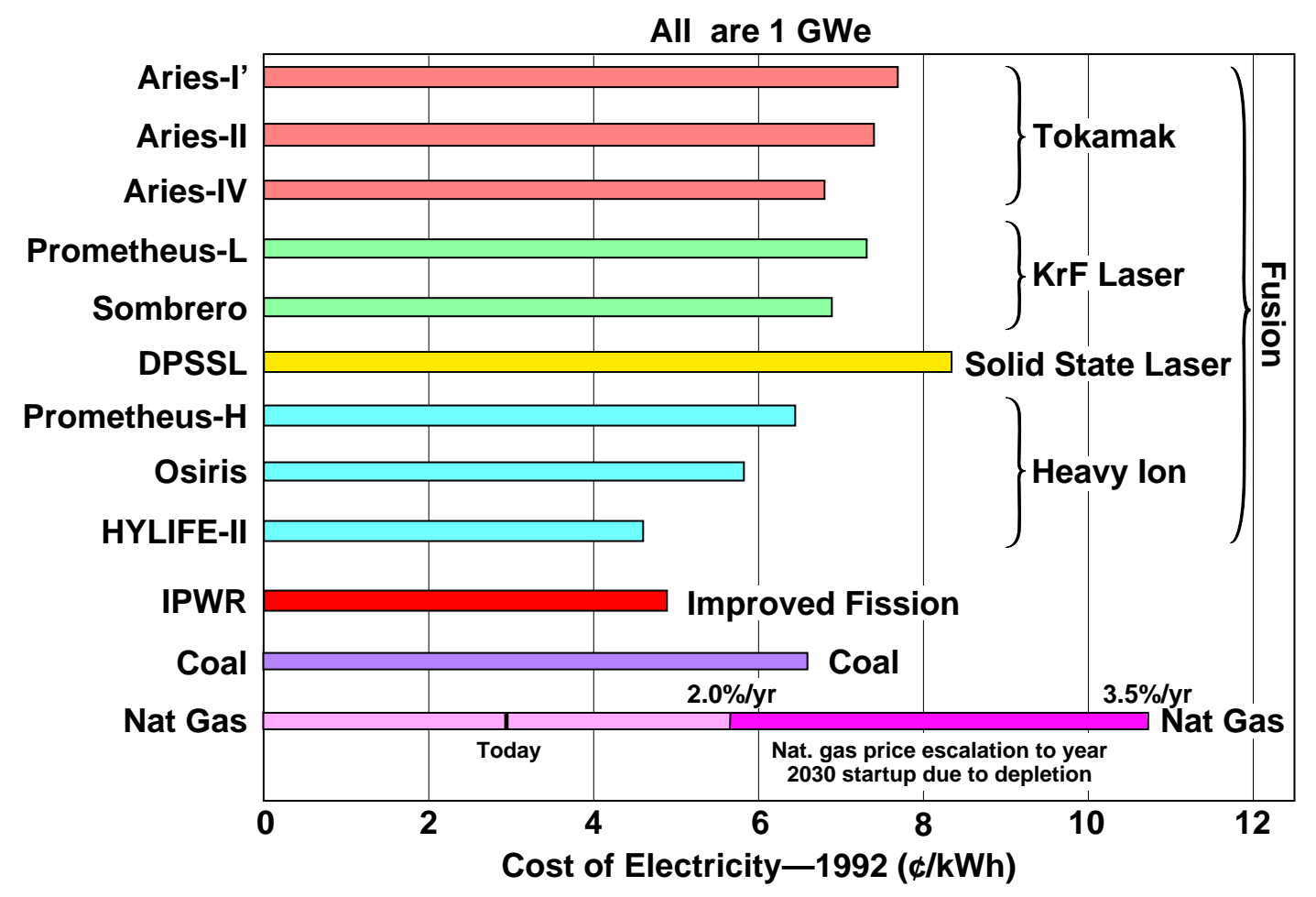

Figure 1.1: The estimated cost of electricity for several conceptual fusion power plant designs in comparison to natural gas, coal, and fission [63].

\subsection{Principles of Fusion}

Research on fusion as an economically viable energy source has been conducted for over four decades now [30], in which tremendous progress has been made. In order for fusion to fulfill its promise of clean and abundant energy, we need to proceed and improve our understanding of the physics involved. To appreciate the difficulties encountered in making fusion a reality, we will first discuss its principles.

Fusion energy is released when two light ions merge to form a heavier one. Fusion reactions commonly considered for energy production are:

$$
\mathrm{D}+\mathrm{T} \rightarrow{ }^{4} \mathrm{He}(3.5 \mathrm{MeV})+\mathrm{n}(14.1 \mathrm{MeV})
$$




$$
\begin{aligned}
\mathrm{D}+\mathrm{D} & \rightarrow \mathrm{T}(1.01 \mathrm{MeV})+\mathrm{p}(3.02 \mathrm{MeV}) \\
\mathrm{D}+\mathrm{D} & \rightarrow{ }^{3} \mathrm{He}(0.82 \mathrm{MeV})+\mathrm{n}(2.45 \mathrm{MeV}) \\
\mathrm{D}+{ }^{3} \mathrm{He} & \rightarrow{ }^{4} \mathrm{He}(3.66 \mathrm{MeV})+\mathrm{p}(14.6 \mathrm{MeV}) \\
\mathrm{T}+\mathrm{T} & \rightarrow{ }^{4} \mathrm{He}+2 \mathrm{n}+11.3 \mathrm{MeV} \\
\mathrm{p}+{ }^{11} \mathrm{~B} & \rightarrow 3{ }^{4} \mathrm{He}+8.68 \mathrm{MeV}
\end{aligned}
$$

If we only consider the products of these fusion reactions, $\mathrm{p}-{ }^{11} \mathrm{~B}$ fusion would be the most attractive candidate for use in power plants. No neutrons are produced in this reaction, which would avoid activation problems in a fusion plant. In addition, the fusion energy is released in the form of fast-moving charged particles, whose energy can be converted into electricity using high-efficiency direct converters.

For a mono-energetic beam with number density $n_{\mathrm{b}}$ and velocity $v$ impinging on a stationary target with number density $n_{\mathrm{t}}$, the number of beam particles that have undergone a fusion reaction while traveling a distance $\mathrm{d} x$ is proportional to the cross section $\sigma(v)$ :

$$
\mathrm{d} n_{\mathrm{b}}=-\sigma(v) n_{\mathrm{b}} n_{\mathrm{t}} \mathrm{d} x=-\sigma(v) n_{\mathrm{b}} n_{\mathrm{t}} v \mathrm{~d} t
$$

in which the cross section $\sigma(v)$ depends on the beam velocity $v$. For a fusion fuel at a temperature $T$, we average the right-hand-side of this equation over the thermal velocity distribution of the fuel. Instead of the cross section at a fixed velocity $v$, we now consider the reaction rate parameter $\langle\sigma v\rangle$, which is the product of the cross section $\sigma$ and velocity $v$ averaged over a Maxwellian velocity distribution.

The reaction rate parameter is plotted as a function of temperature in figure 1.2. The DT reaction has the largest reaction rate parameter and is therefore usually considered 


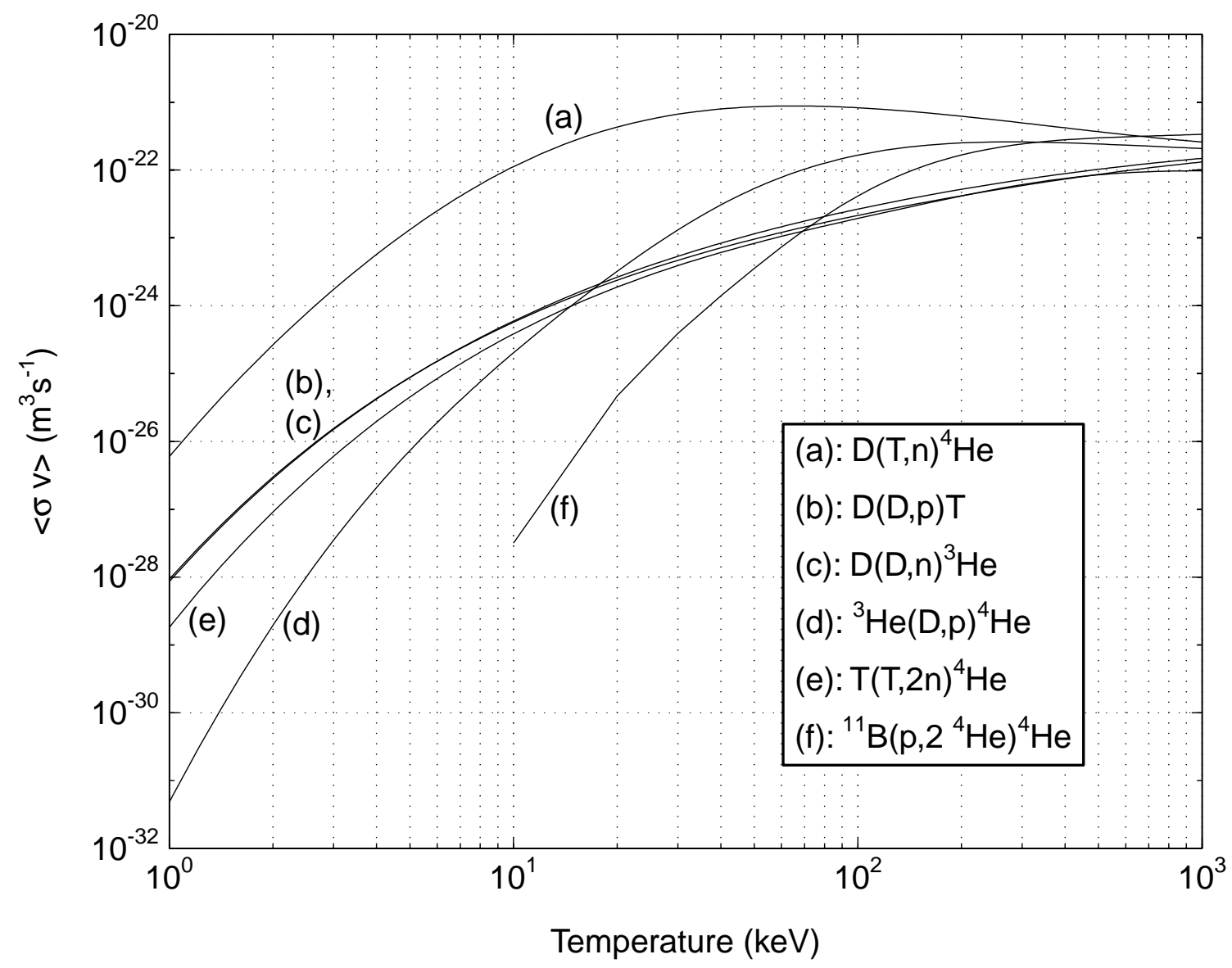

Figure 1.2: Reaction rate parameters for Maxwellian distributions as a function of temperature. These data were taken from the DATLIB cross section and reactivity parameter library [29].

to be the prime candidate for commercial fusion plants. The reaction rate parameter reaches a maximum at a temperature of $20 \mathrm{keV}$. At such high temperatures, the deuterium and tritium fuel will be ionized and form a plasma. The two DD reactions can significantly contribute to the fusion power generated in DT fuel. Unfortunately, the reaction rate parameter for $\mathrm{p}^{-11} \mathrm{~B}$ fusion is smaller by orders of magnitude.

Suppose we have a fuel consisting of an equal mixture of deuterium and tritium with initial combined density $n_{0}$. The burnup fraction $f_{\mathrm{b}}(t)$ is defined in terms of the fuel 
density $n(t)$ at time $t$ by $n(t)=n_{0}\left(1-f_{\mathrm{b}}(t)\right)$. It can be calculated from equation (1.1) as

$$
f_{\mathrm{b}}(t)=\frac{\frac{1}{2} n_{0}\langle\sigma v\rangle t}{\frac{1}{2} n_{0}\langle\sigma v\rangle t+1} .
$$

This equation shows that if we are able to confine a burning plasma longer, we will improve the burnup fraction of the fuel and thereby the fusion plant economics. The burnup time $\tau_{\mathrm{b}}$ is defined as

$$
\tau_{\mathrm{b}} \equiv\left[n_{0}\langle\sigma v\rangle\right]^{-1}
$$

Our aim is to confine a burning plasma on a time scale comparable to the burnup time.

There are three ways to confine and heat a plasma sufficiently in order for fusion to take place. Whereas gravitational confinement enables fusion to occur in stars, the two main approaches for man-made fusion are based on magnetic or inertial confinement.

In magnetic fusion energy (MFE), a burning fusion plasma is confined using strong magnetic fields, which are created by external coils as well as by electric currents flowing in the fusion plasma itself. Since magnetic fields exert only transverse forces on charged particles, they can move freely along magnetic field lines, while their motion across field lines is impeded. By bending the field lines around in a toroidally shaped fusion reactor (tokamak), the loss of plasma ions from the machine can be minimized. In addition, the reduced transverse mobility of the plasma ions prevents heat leaking away from the fusion plasma. A conceptual drawing of the DIII-D tokamak of General Atomics is shown in figure 1.3 .

In practice, various instabilities occur in magnetically confined plasmas. Macroscopic distortions of the plasma are called magnetohydrodynamic (MHD) instabilities, whereas growing waves in the plasma are referred to as microinstabilities. Both may cause 


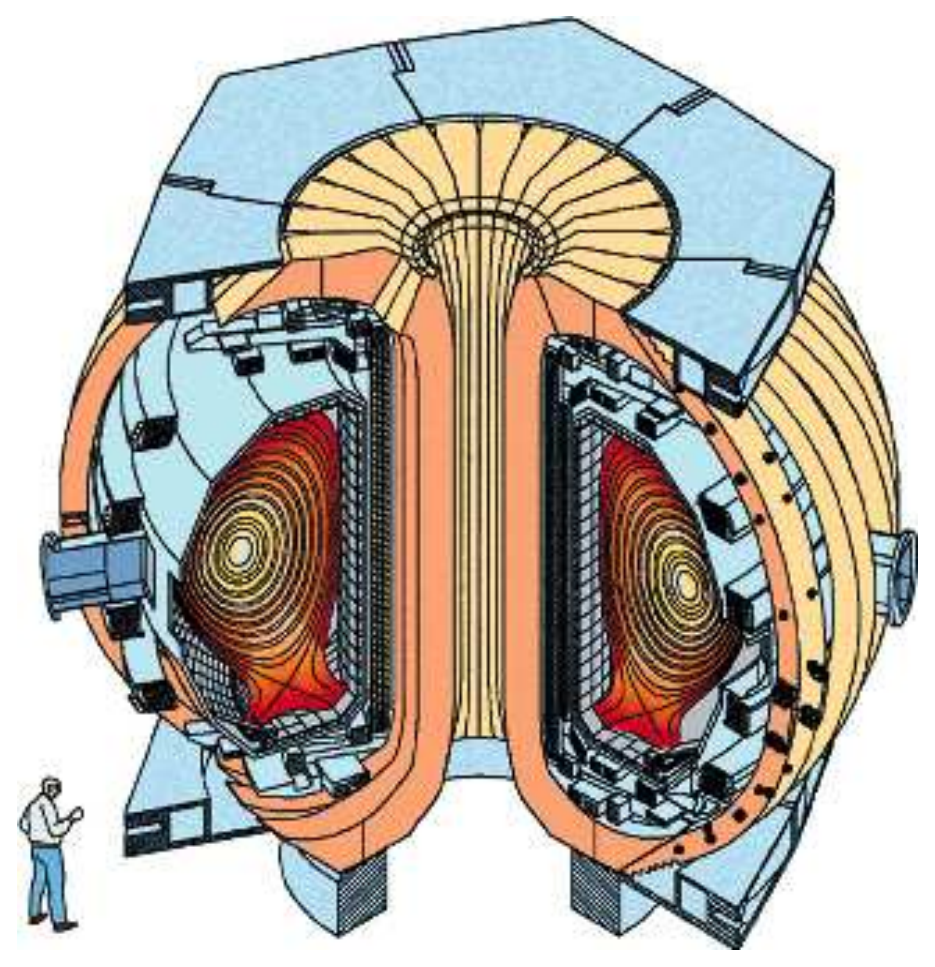

Figure 1.3: The DIII-D Tokamak at General Atomics. Figure used with permission of General Atomics.

an increase in the heat conduction and particle diffusion rate across magnetic field lines.

In addition, various engineering problems need to be solved before magnetic fusion can become a viable source of energy. The magnet coils in a tokamak reactor need to be strong enough to confine the burning plasma, and should be able to withstand the tremendous pressures exerted on them by the magnetic field. The need for cooling is an additional difficulty when using superconducting magnets. Maintenance may be difficult in the presence of activation by the $14 \mathrm{MeV}$ neutrons produced in DT fusion. Heat loss from the plasma due to radiation by plasma impurities can significantly lower the plasma temperature. The bombardment of the reactor wall by high-energy alpha particles can cause blisters, which eventually rupture and lead to plasma contamination. Finally, the 
need for plasma stability requires a large machine size, which is economically unattractive.

In inertial fusion energy (IFE), a small pellet of DT fuel is compressed to very high densities by heating it with a laser or ion beam. In case of ion beams, either light ions or heavy ions can be used [58]. A hot spot in the center of the fuel pellet, containing only a fraction of the total fuel mass, is heated during compression to about 5 to $10 \mathrm{keV}$, causing fusion to occur. Once the hot spot is ignited, the alpha particles produced in the fusion reactions heat up the fuel surrounding the hot spot, enabling a fusion burn wave to propagate outward from the hot spot. Due to the inertia of the fuel, the fuel pellet will remain compressed long enough for a substantial fraction of the fuel to undergo fusion. By igniting pellets several times per second, sufficient fusion energy can be released for commercial electricity production.

Figure 1.4 shows a diagram of the energy flow in a power plant based on inertial fusion [86]. Assuming a driver efficiency $\eta_{\mathrm{D}}$, the input energy $E_{\text {in }}$ is used by the driver to produce a high-power laser or ion beam with energy $\eta_{\mathrm{D}} E_{\mathrm{in}}$. The yield is defined as the fusion energy released from one fuel pellet, while the gain $G$ is the ratio of the yield to the required driver energy. The yield can then be written as $G \eta_{\mathrm{D}} E_{\mathrm{in}}$, and is converted into electricity in a conventional steam cycle with thermal efficiency $\eta_{\mathrm{Th}}$. A fraction $f$ of the generated electricity $E_{\text {out }}=\eta_{\mathrm{Th}} G \eta_{\mathrm{D}} E_{\text {in }}$ is recycled to the driver, so that the next fuel pellet can be ignited.

From this flow diagram, we find that $f \eta_{\mathrm{Th}} G \eta_{\mathrm{D}}=1$ is a condition for continuous operation of the power plant. For a recycling fraction $f$ less than $25 \%$, and assuming a thermal efficiency of $40 \%$, we find that the product $\eta_{\mathrm{D}} G$ should be greater than ten [86]. 


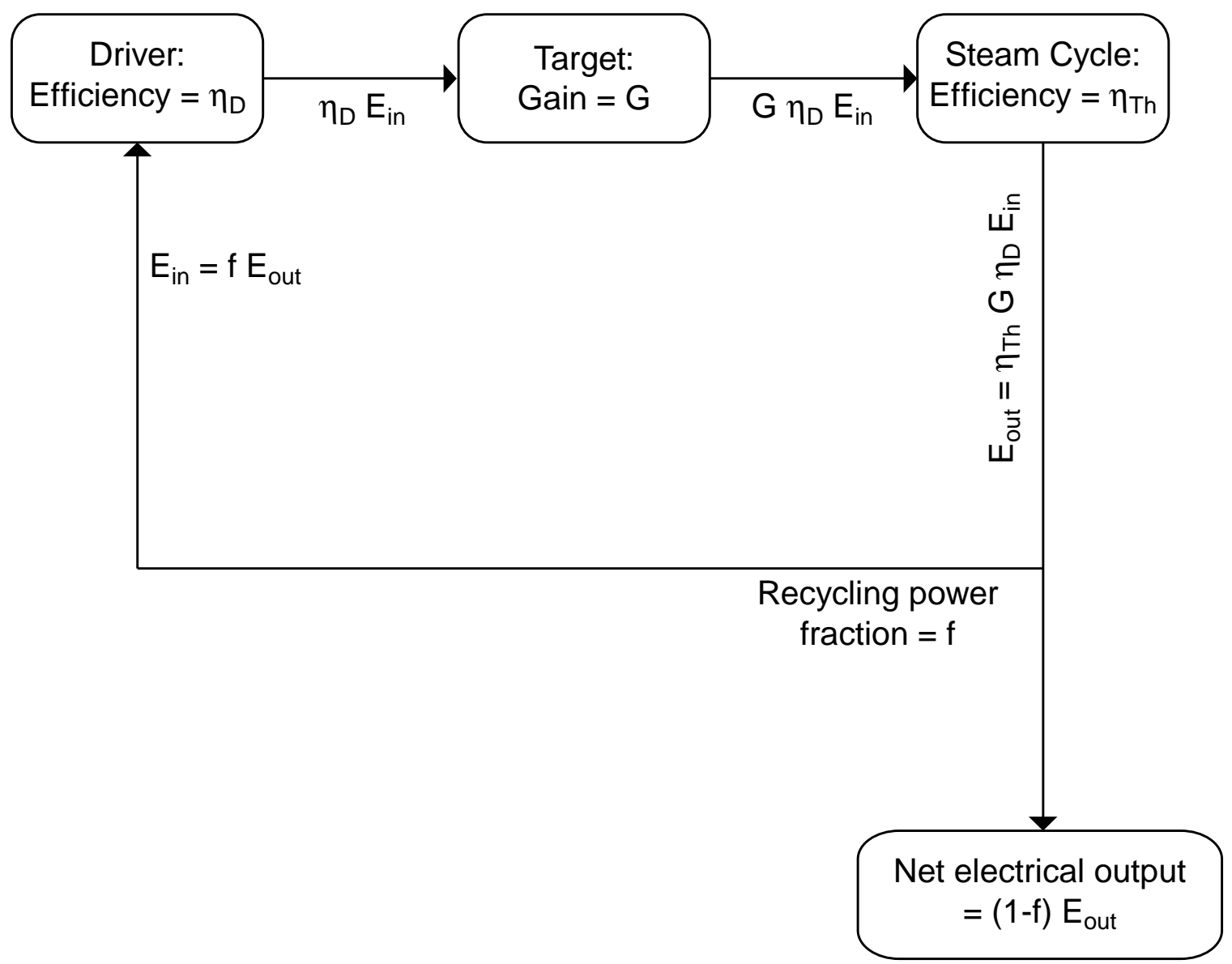

Figure 1.4: Energy flows in a power plant based on inertial fusion [86].

For contemporary target designs, a yield of $430 \mathrm{MJ}$ at a driver energy of $6.5 \mathrm{MJ}$ has been predicted from simulations [93]. Typically a heavy-ion accelerator has an efficiency of about $25 \%$, giving a value of $\eta_{\mathrm{D}} G$ equal to 16 . If we are able to ignite fuel pellets at a rate of $5 \mathrm{~Hz}$, we can generate $2150 \mathrm{MW}$ thermal energy, which corresponds to $860 \mathrm{MW}$ electric energy for a thermal efficiency of $40 \%$.

Inertial fusion micro-explosions can be driven directly or indirectly. In case of directly driven targets, the laser or ion beam hits the DT fuel itself and compresses it. In indirect drive, the DT fuel pellet is placed inside a cylindrically symmetric casing usually 


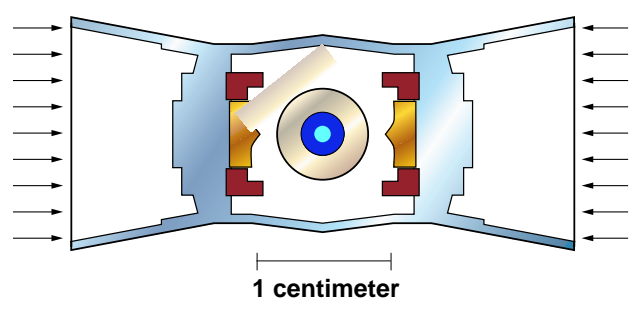

Figure 1.5: A hohlraum for heavy-ion driven IFE, showing a DT fuel pellet inside a cylindrical casing lined with metal [63].

referred to by its German term hohlraum. Figure 1.5 shows a conceptual diagram of a hohlraum. In the case of laser fusion, the laser beams enter through holes on both sides of the hohlraum and deposit their energy on the inside of its walls. The heated walls create a uniform x-ray radiation field inside the hohlraum, which then compresses the fuel pellet. Hohlraums for use with ion-driven inertial fusion typically have radiation converters on both ends. The ion beams are stopped by the converter material and deposit their energy there. The heated converter material then emits x-rays, which produce the uniform radiation field inside the hohlraum required to compress the fuel pellet. Indirect drive has the advantage that spherically uniform compression can be achieved using only a limited number of beams. In general, direct drive requires a larger number of beams in order to heat the target from all sides and compress it uniformly.

After compression of the fuel pellet, a rarefaction wave propagates inward at the sound speed $c_{\mathrm{s}}$. For equal ion and electron temperature $T$, the sound speed is given by

$$
c_{\mathrm{s}}^{2}=\frac{\gamma \cdot 2 T}{m_{\mathrm{ion}}}
$$

in which $\gamma=\frac{5}{3}$ is the ratio of the heat capacity at constant pressure and at constant volume. A rarefaction wave that starts at the pellet radius $R$ reaches the radial distance $r<R$ in 
a time $(R-r) / c_{\mathrm{s}}$. Assuming a uniform density, we can average this time span over the volume of the pellet to find the confinement time:

$$
\tau_{\mathrm{c}}=\frac{1}{\frac{4}{3} \pi R^{3}} \int_{0}^{R} 4 \pi r^{2}\left(\frac{R-r}{c_{\mathrm{s}}}\right) d r=\frac{R}{4 c_{\mathrm{s}}} .
$$

From equations (1.4) and (1.5) we can calculate the burnup fraction given in equation (1.2). We find

$$
f_{\mathrm{b}}=\frac{1}{1+\frac{8 c_{\mathrm{s}}}{n_{0}\langle\sigma v\rangle R}}=\frac{1}{1+\frac{8 c_{\mathrm{s}} \cdot 2.5 \mathrm{amu}}{\rho\langle\sigma v\rangle R}},
$$

in which $2.5 \mathrm{amu}$ is the average mass per ion of the DT fuel. At a temperature of $30 \mathrm{keV}$, the quantity $8 \cdot(2.5 \mathrm{amu}) c_{\mathrm{s}} /\langle\sigma v\rangle$ reaches a minimum value of about $70 \mathrm{~kg} \mathrm{~m}^{-2}[86]$. At this temperature, the burnup fraction is

$$
f_{\mathrm{b}}=\frac{\rho R}{\rho R+70 \mathrm{~kg} \mathrm{~m}^{-2}}
$$

In order to overcome inefficiencies in other parts of an IFE power plant, a burnup fraction of about $30 \%$ is required. For a yield of $430 \mathrm{MJ}$, this corresponds to a mass of $4.2 \mathrm{mg}$ of DT fuel in one pellet. At a $\rho R$ of $30 \mathrm{~kg} \mathrm{~m}^{-2}$, the fuel density is $1.6 \cdot 10^{5} \mathrm{~kg} \mathrm{~m}^{-3}$, while the pellet radius after compression is about $180 \mu \mathrm{m}$. At a temperature of $30 \mathrm{keV}$, we find the confinement time from equations (1.4) and (1.5) to be equal to $35 \mathrm{ps,} \mathrm{while} \mathrm{the} \mathrm{burnup}$ time can be calculated from equation (1.3) to be about 40 ps.

The density of frozen DT is about $200 \mathrm{~kg} \mathrm{~m}^{-3}$, so the fuel pellet needs to be compressed by a factor of almost 1000. If the pellet undergoes ideal spherical compression, its radius is reduced by a factor of 10 . In practice however, due to the occurrence of the Rayleigh-Taylor and other instabilities, the compression is not perfectly spherical. This leads to an increase of the surface to volume ratio of the pellet during compression. As a 
result, the need for radial compression is increased to about 20 to 30 , corresponding to an initial pellet radius of 3.6 to $5.4 \mathrm{~mm}[86]$.

The energy needed for compression will be minimized if the compression takes place adiabatically to avoid heating the DT fuel. The hot spot does need to be heated though, which asks for a careful design of the fuel configuration with different values for $\rho R$ in different parts of the pellet. In addition, we need to be able to shape the driver beam energy as a function of time in order to optimize compression. In contemporary target designs based on heavy ion inertial fusion, a beam pulse consisting of a prepulse of $1.5 \mathrm{MJ}$ and a main pulse of 4.4 MJ is envisioned [20]. Obtaining the optimal pulse shape for an ion beam or laser pulse will prove to be a difficult task and puts additional requirements on the accelerator or laser system.

\subsection{Heavy Ion Inertial Fusion}

In heavy ion inertial fusion, the DT target implosion is driven by a beam of heavy ions at an energy of a few to several GeV. Heavy ion fusion has several advantages over laser driven fusion. First, accelerator technology has benefited from decades of engineering experience from accelerators for high-energy physics experiments, resulting in a high reliability. Accelerators have a long lifetime of the order of decades, and have high efficiencies of around $30 \%$, as we would need for a fusion power plant. The required repetition rate of several per second is easily achievable in accelerators, in contrast to high-intensity lasers. Small focal spots on the target are more easily attainable with lasers than with ion beams though. Furthermore, it should be realized that the heavy ion accelerators needed for fusion 
applications have a significantly higher beam current than currently existing accelerators. In particular, we need a better understanding of nonlinear space-charge forces, which are virtually absent in present low-current accelerators.

In the contemporary target design mentioned above, the prepulse delivers 1.5 MJ of $3 \mathrm{GeV}$ lead ions, while the main pulse delivers $4.4 \mathrm{MJ}$ of $4 \mathrm{GeV}$ lead ions. The prepulse and the main pulse can be accelerated in the same accelerator up to an energy of $3 \mathrm{GeV}[20]$. The prepulse would last almost $30 \mathrm{~ns}$, whereas the pulse duration of the main pulse is about 10 ns. The choice of beam ion depends on its characteristics concerning range and energy deposition profile in the target material. Lead, cesium and mercury are common candidates. Nowadays usually single-charged ions are considered because of the increased space-charge repulsion of higher-charged beam ions.

Whereas radiofrequency linear accelerators (RF linacs) are considered the prime candidate for heavy-ion inertial fusion in Europe and Japan, in the United States the focus has been on induction linacs. RF linacs make use of resonant cavities as their basic accelerating structure. The beam is accelerated by generating RF waves in the cavities at a phase such the beam ions experience a forward accelerating electric field as they are passing through. In induction linacs, the beam passes through pulsed toroidal magnets by which a long pulse accelerating electric field is generated. By ramping the current flowing around the induction cores, the magnetic flux in the induction cores increases as a function of time. Following Faraday's law, we find a longitudinal electric field which accelerates the beam. The product of acceleration and pulse duration that can be achieved in one induction cell is limited due to saturation of the magnetic field in the induction core. 
Conventional accelerators transversely confine the beam using electrostatic and magnetic lenses, while the longitudinal beam velocity is increased in a sequence of accelerating gaps between those lenses. Since the magnetic focusing force $q e \vec{v} \times \vec{B}$ is proportional to the ion velocity $v$, the beam energy has to be sufficiently high for magnetic focusing to be effective in opposing the space-charge force.

Existing designs for heavy ion accelerators for inertial fusion typically look like the diagram shown in figure 1.6. After leaving the ion source, the beam is matched into the accelerator, in which the beam energy is increased to about $100 \mathrm{MeV}$. In this lowenergy part of the accelerator, the beam is confined with electrostatic focusing. Beyond this point, magnetic focusing is used. Between the electrostatic section and the magnetic section, or at some other point in the accelerator, beam merging may be employed to optimize the accelerator economics. After the magnetic section of the accelerator, the beam enters the drift compression section, in which it is longitudinally compressed to reduce the pulse duration to several tens of nanoseconds needed on target. Longitudinal compression is achieved by accelerating the tail of the beam to a higher velocity than the head. In the final focus section, the beam is first expanded and then focused onto a spot with a radius of several millimeters. Since significant beam manipulations take place in the final focus system, and since the beam current reaches a maximum there due to acceleration and compression, a proper design of the final focus section is crucial. As the beam enters the reactor chamber, it may be partially or fully neutralized by passing it through a plasma or by coinjecting electrons. Neutralization may be needed at a beam energy of several GeV; for higher beam energies, unneutralized ballistic focusing may be employed. The number of 


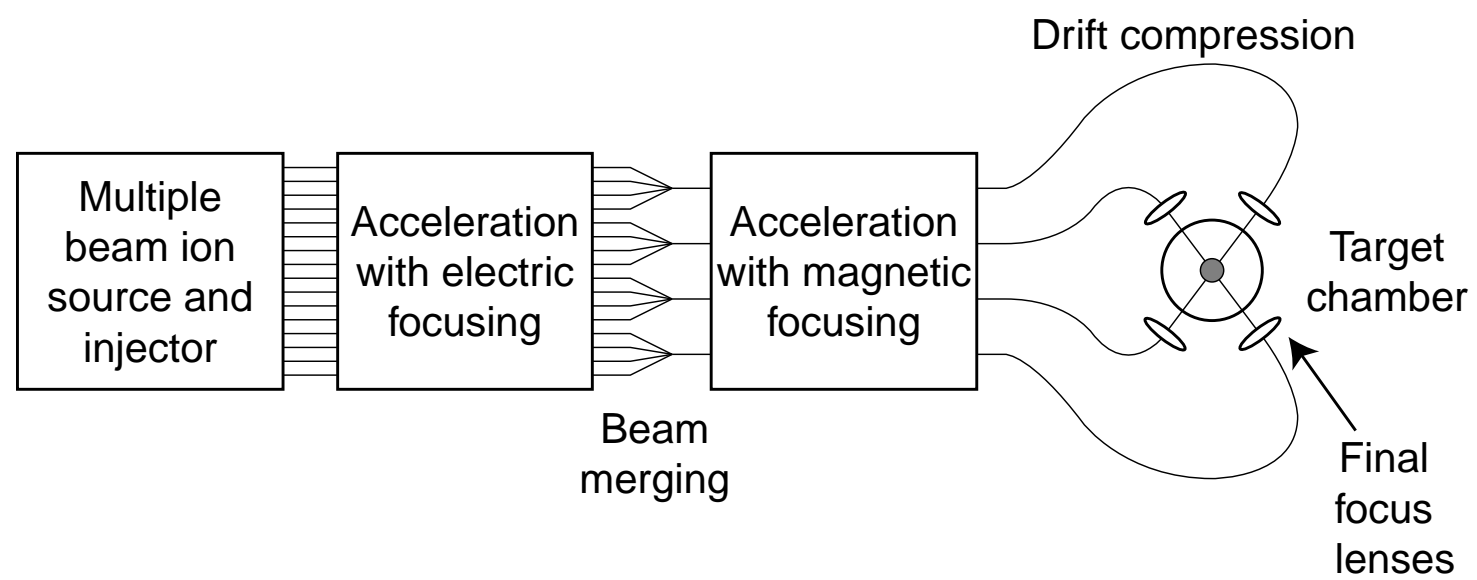

Figure 1.6: Conceptual diagram of a heavy-ion linear accelerator for IFE [6].

beams hitting the target varies between the existing designs and depends on whether direct or indirect drive is used.

For a 1.5 MJ prepulse of $3 \mathrm{GeV}$ ions, divided into sixteen beams, the average beam current will be about one kA per beam for a pulse duration of $30 \mathrm{~ns}$. The average beam current in the 4.4 MJ main pulse of $4 \mathrm{GeV}$ delivered in $10 \mathrm{~ns}$ will be about $3.4 \mathrm{kA}$ when using 32 beams [20].

\subsection{Overview of Conceptual Heavy Ion Drivers and Experi-}

\section{ments}

Typical power plant reactors produce an electrical power of 800 to 1500 MWe. Conceptual designs for heavy-ion driven inertial fusion power plants are usually based on an electrical output of about 1000 MWe. The HIBALL-II and the HYLIFE-II designs to be discussed here are the two most detailed conceptual fusion plant designs for heavy-ion 


\begin{tabular}{|l||c|c|}
\hline & HIBALL-II & HYLIFE-II \\
\hline Number of reactor chamber & 4 & 1 \\
\hline Fusion power (MW) & $8000(2000)$ & 2835 \\
\hline Net electrical output (MW) & $3784(946)$ & 1083 \\
\hline Driver energy (MJ) & 5.0 & 5.0 \\
\hline Target gain & 80 & 70 \\
\hline Fusion yield (MJ) & 400 & 350 \\
\hline Driver efficiency & 0.27 & 0.20 \\
\hline Thermal-to-electrical efficiency & 0.42 & 0.412 \\
\hline Recirculating power fraction & 0.12 & 0.21 \\
\hline Repetition rate (Hz) & $20(5)$ & 8.2 \\
\hline
\end{tabular}

Table 1.1: Plant parameters of the HYBALL-II and HYLIFE-II conceptual power plant designs [35], [75], [96]. Quantities between parentheses indicate the number per reactor chamber.

inertial fusion that have been published. Table 1.1 gives an overview of the power plant parameters for these designs.

\subsubsection{HIBALL-II Design}

The HIBALL-II design by the Kernforschungszentrum Karlsruhe (Germany) originated in 1985 in cooperation with the University of Wisconsin [35] and is an improvement of the original HIBALL design [4]. The driver in this design consists of an RF linac combined with transfer, storage and buncher rings. It operates at a repetition rate of $20 \mathrm{~Hz}$, driving four reactor chambers at $5 \mathrm{~Hz}$ each. In principle, the driver could achieve a higher repetition rate and drive even more reactor chambers [96].

The driver accelerates $\mathrm{Bi}^{+}$beams of $1.25 \mathrm{kA}$ each to a kinetic energy of $10 \mathrm{GeV}$ over a length of $5 \mathrm{~km}$. The final focusing magnets, which are located just outside the reactor chamber, focus the beams ballistically without neutralization over a standoff distance of 8.5 meters onto a $7 \mathrm{~mm}$ diameter spot on the fuel pellets. These pellets are directly driven 
by twenty beams each, giving a yield of $400 \mathrm{MJ}$ at a gain of 83 [96].

The fusion energy is absorbed by the blanket structures and the coolant, which consists of $83 \%$ lead and $17 \%$ lithium. The coolant transfers the heat to the heat exchangers and steam generators for electricity production. The tritium needed for the fusion reactions is produced in neutron absorption reactions in the lithium present in the coolant:

$$
{ }^{6} \mathrm{Li}+\mathrm{n} \rightarrow \mathrm{T}+{ }^{4} \mathrm{He} .
$$

In addition to tritium breeding, the coolant serves the purpose of protecting the reactor chamber against neutron activation.

\subsubsection{HYLIFE-II Design}

The HYLIFE-II design is a conceptual design for an inertial fusion power plant driven by heavy-ion beams or high-power lasers [75]. HYLIFE-II focuses mainly on the reactor chamber design and engineering instead of on the driver. The fuel target though is designed specifically to be driven by heavy ions such as ${ }^{200} \mathrm{Hg}^{+}$at $10 \mathrm{GeV}$. Figure 1.7 shows the conceptual design of the HYLIFE-II chamber.

The main improvement of HYLIFE-II over the original HYLIFE-I design [11] is the use of a thick liquid wall of Flibe as a coolant instead of liquid lithium because of its fire hazard, lower neutron attenuation, and higher vapor pressure. Flibe is a molten salt composed of $\mathrm{LiF}$ and $\mathrm{BeF}_{2}$. In the HYLIFE-II target chamber, arrays of nozzles generate oscillating jets of liquid Flibe, creating a cavity through which the heavy ion beams pass to ignite a target in the center of the cavity. As in case of HIBALL-I, the coolant serves the additional purposes of tritium breeding and neutron shielding of chamber structures. 


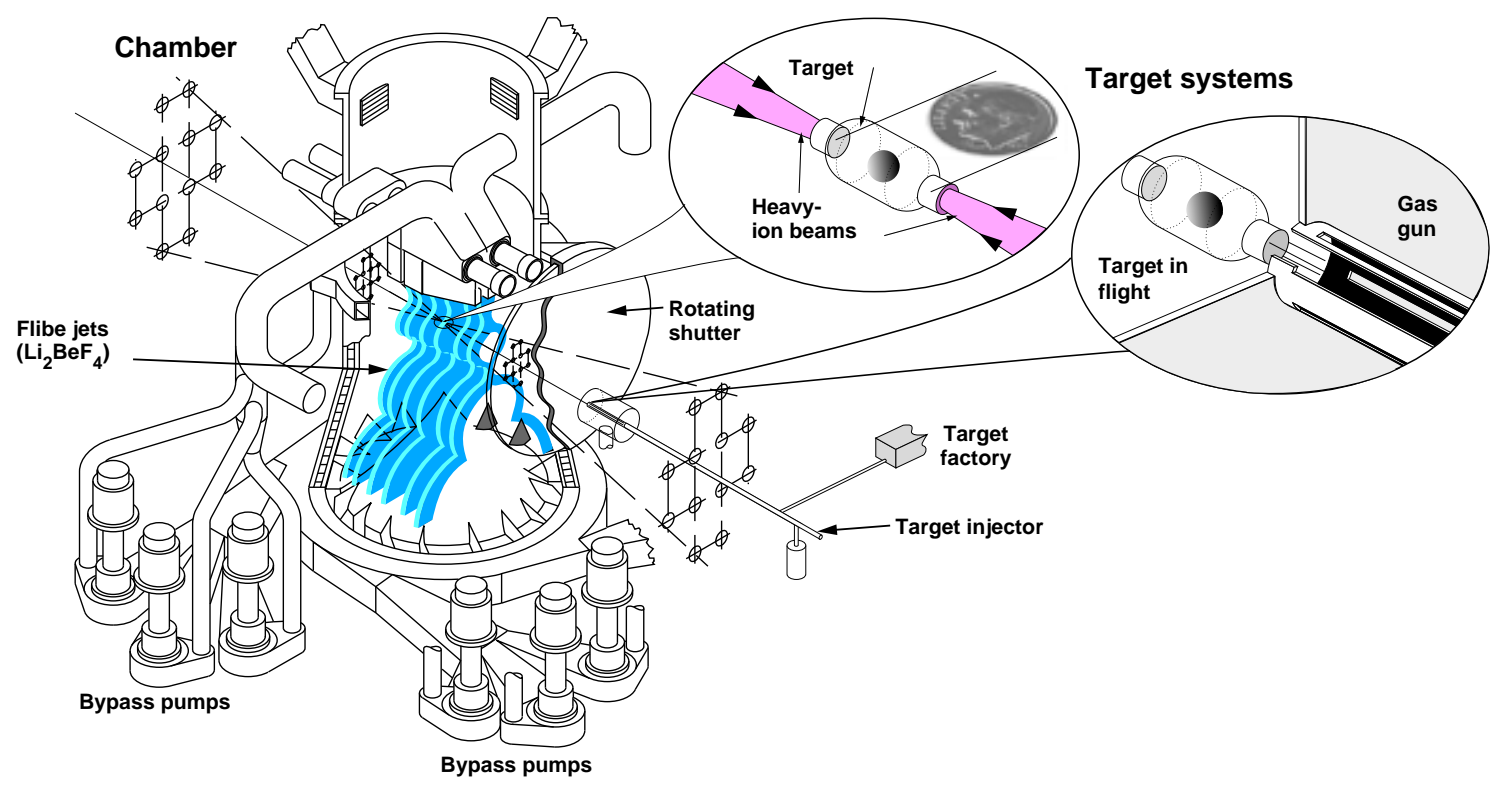

Figure 1.7: The HYLIFE-II chamber.

\subsubsection{Small and Intermediate Scale Experiments}

\section{The Scaled Final Focus Experiment}

In the Scaled Final Focus Experiment at LBNL [68], [69], the beam dynamics of the HIBALL-II final focus system are replicated on a small scale. All spatial dimensions were reduced by a factor of ten, while the other beam parameters were scaled appropriately. The Scaled Final Focus Experiment uses a $\mathrm{Cs}^{+}$ion beam accelerated to an energy of $160 \mathrm{keV}$.

The focusing lattice consists of a 2.2 meter section of fifteen electrostatic quadrupoles, followed by a drift space, and six magnetic quadrupoles. The drift space between the third and fourth magnet was reduced with respect to the HIBALL-II design, since its bending magnets, which are needed for the neutron dump, were not included in the small-scale experiment. 


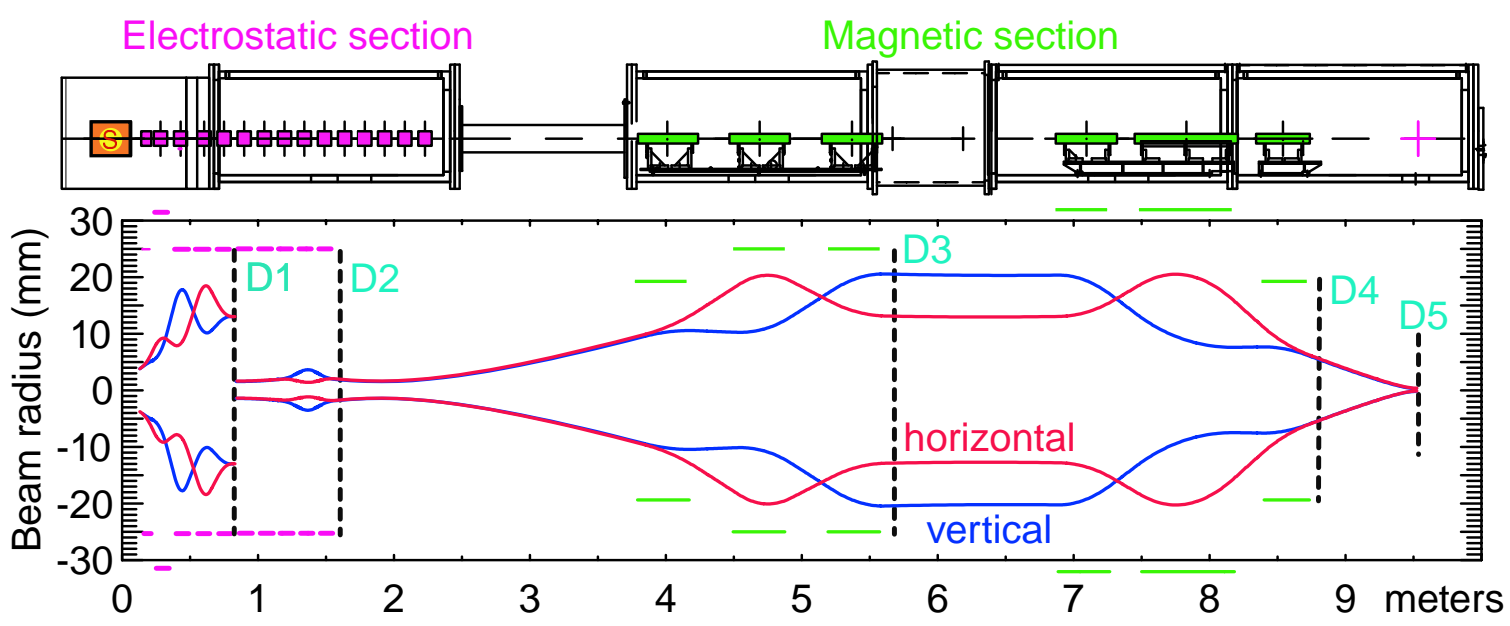

Figure 1.8: The Scaled Final Focus Experiment.

Upon exit from the diode, the $\mathrm{Cs}^{+}$beam has a current of several $\mathrm{mA}$ and is transported through the first five electrostatic quadrupoles to an aperture plate. The beam passes through a hole several millimeters wide in the aperture plate to reduce the current to a value typically between 80 and $400 \mu \mathrm{A}$, depending on the size of the aperture hole. After the aperture, the second set of five electrostatic quadrupoles match the beam to the beam radius and convergence angle required for the actual final focus section, which consists of the six magnetostatic quadrupoles. The last five electrostatic quadrupoles, before the magnetic section, were kept at zero field in the experiment.

Figure 1.8 shows a schematic of the Scaled Final Focus Experiment. The upper part of the figure is a drawing to scale of the beam lattice showing the electric and magnetic quadrupoles, whereas the lower part shows the horizontal and vertical beam radius. The location of the diagnostic probes, which are used to measure the phase-space properties of the beam, are represented by the dashed lines. The dashed vertical line after the fifth electrostatic quadrupole represents the location of the aperture. Since the last five electrostatic 
quadrupoles were usually set to ground during the experiments, they are not drawn on the lower part of figure 1.8.

\section{The High Current Experiment (HCX)}

The High Current Experiment (HCX) will be the first beam transport experiment in which the beam has a duration and line charge density that correspond to driver-scale values [87]. The HCX will consist of a single low-energy $(1.6-2 \mathrm{MeV}) \mathrm{K}^{+}$beam with a long pulse duration ( $\sim 5-20 \mu \mathrm{s})$, focused using electrostatic and magnetic quadrupoles. In the HCX, important beam physics issues such as preservation of beam quality, beam sensing and alignment, halo production, and secondary electron effects will be addressed for high-intensity beams. In particular, the aperture fill factor as defined as the ratio of the beam radius to the pipe radius will be studied. The HCX is aimed to provide a sound knowledge basis to proceed with the IRE, discussed below.

\section{The Integrated Research Experiment (IRE)}

The Integrated Research Experiment (IRE) is envisioned to be the next major step in the US inertial fusion energy program [7]. In the IRE, beam dynamics issues will be studied such as emittance growth, halo formation, pulse compression, beam loading (the effect of the beam on the accelerator circuitry) and the use of multiple beams, as well as chamber transport and final focus systems including neutralization, beam stability, and stripping and photoionization issues. Furthermore the IRE can be used to examine ion beam interaction with targets, such as volumetric heating of matter (which is unique to energy deposition by ion beams in comparison to laser beams) and ion stopping power in 
dense plasmas. The objective of the IRE is to provide the scientific and technological basis to proceed to design and build an Engineering Test Facility (ETF) for inertial fusion.

Current preliminary designs for an IRE are based on realizing these goals [7]. The target temperature that can be achieved when the beams hit the target depends on the beam energy, the beam current, the final pulse duration and the spot size on target. To make meaningful final focus experiments possible, the ion energy should be greater than about $100 \mathrm{MeV}$. The total current at the end of the IRE accelerator should be larger than about 100 A for beam loading to have similar effects as in a driver. This large total current will require the use of tens of beams. In addition, the parameters of the IRE should be such that it can serve as a stepping stone between small-scale experiments $(1 \mathrm{~A}$ beams at a few $\mathrm{MeV}$ ) and an ETF (100 A beams at a few $\mathrm{GeV}$ ). The exact parameters of the IRE will result from a better knowledge of its requirements.

Preliminary designs for the IRE have been made and form the basis of some of the simulation results presented in this thesis. The beam parameters for the current conceptual IRE design are listed in table 1.2, together with the corresponding values for a typical power-plant scale driver [74]. In order to be able to focus the beams onto a small spot, neutralizing the beam with a neutralization fraction of $90 \%$ or higher is required. The IRE parameters given in table 1.2 will yield a target temperature of about $43 \mathrm{eV}$ if the beams is focused onto a spot with a $5 \mathrm{~mm}$ radius [7].

In current designs, the IRE is envisioned to consist of an injector and matching section of six meters, an electrostatically focused accelerator of 50 meters followed by a magnetically focused accelerator of 205 meters, a drift compression section of 60 meters 


\begin{tabular}{|l|c|c|}
\hline & IRE & Power plant \\
\hline \hline Number of beams & 32 & 140 \\
\hline Charge per beam & $4.6875 \mu \mathrm{C}$ & $32.33 \mu \mathrm{C}$ \\
\hline $\begin{array}{l}\text { Current per beam at the } \\
\text { end of the accelerator }\end{array}$ & $15.3 \mathrm{~A}$ & $157 \mathrm{~A}$ \\
\hline $\begin{array}{l}\text { Final kinetic } \\
\text { energy }\end{array}$ & $200 \mathrm{MeV}$ & $\begin{array}{l}1.1 \mathrm{GeV} \text { prepulse; } \\
1.4 \mathrm{GeV} \text { main pulse }\end{array}$ \\
\hline Normalized emittance & $<5 \mathrm{~mm} \mathrm{mrad}$ & $0.8 \mathrm{~mm}$ mrad \\
\hline Momentum spread & $<1 \%$ & $\begin{array}{l}0.43 \% \text { prepulse; } \\
1.3 \% \text { main pulse }\end{array}$ \\
\hline $\begin{array}{l}\text { Pulse duration } \\
\text { at the end of the accelerator }\end{array}$ & $335 \mathrm{~ns}$ & $205 \mathrm{~ns}$ \\
\hline $\begin{array}{l}\text { Pulse duration } \\
\text { after compression }\end{array}$ & $50 \mathrm{~ns}$ & $\begin{array}{l}30 \mathrm{~ns} \text { prepulse; } \\
8 \mathrm{~ns} \text { main pulse }\end{array}$ \\
\hline
\end{tabular}

Table 1.2: Beam parameters for the preliminary IRE design, and for a typical power plant based on Heavy Ion Inertial Fusion.

in which the beam is compressed longitudinally, and a target chamber. The magnetically focused accelerator consists of two sections. In the first section, the beam undergoes longitudinal bunch compression, whereas in the second section the bunch length is held constant and the acceleration gradient is at its maximum value. Table 1.3 gives an overview of the beam parameters in the various parts of the accelerator.

The conceptual design for the IRE is currently in its preliminary stage, and will require further validation and optimization. A number of computer simulations of the IRE have been carried out so far to examine its viability [21], [32], [59].

\subsection{Purpose of Thesis}

Focusing a high-current ion beam onto a small spot on the target is a difficult task, requiring significant beam manipulations by the final focus system. Under these extreme conditions, any imperfections present in the system may severely affect the beam quality. 


\begin{tabular}{|l||c|c|c|c|c|c|}
\hline & $\begin{array}{c}\text { Bunch } \\
\text { length }\end{array}$ & $\begin{array}{c}\text { Lattice } \\
\text { half period }\end{array}$ & $\begin{array}{c}\text { Beam } \\
\text { current }\end{array}$ & $\begin{array}{c}\text { Occu- } \\
\text { pancy }\end{array}$ & $\begin{array}{c}\text { Accelerating } \\
\text { gradient }\end{array}$ & $\begin{array}{c}\text { Focusing } \\
\text { gradient }\end{array}$ \\
\hline$T=1.6 \mathrm{MeV}$ & $18.8 \mathrm{~m}$ & $0.21 \mathrm{~m}$ & $0.77 \mathrm{~A}$ & 0.65 & $55 \mathrm{kV} / \mathrm{m}$ & $140 \mathrm{MV} / \mathrm{m}^{2}$ \\
\hline $\begin{array}{l}\text { Electrostatic } \\
\text { focusing }\end{array}$ & constant & $\propto T^{\frac{1}{2}}$ & $\propto T^{\frac{1}{2}}$ & 0.65 & $\propto T$ & $140 \mathrm{MV} / \mathrm{m}^{2}$ \\
\hline$T=9.4 \mathrm{MeV}$ & $18.8 \mathrm{~m}$ & $0.5 \mathrm{~m}$ & $1.87 \mathrm{~A}$ & jump & $323 \mathrm{kV} / \mathrm{m}$ & jump \\
\hline $\begin{array}{l}\text { Magnetostatic } \\
\text { focusing with } \\
\text { compression }\end{array}$ & $\propto T^{-\frac{1}{2}}$ & $\propto T^{\frac{1}{4}}$ & $\propto T$ & 0.33 & $\propto T$ & $37 \mathrm{~T} / \mathrm{m}$ \\
\hline$T=29.8 \mathrm{MeV}$ & $10.6 \mathrm{~m}$ & $0.67 \mathrm{~m}$ & $5.9 \mathrm{~A}$ & 0.33 & $1000 \mathrm{kV} / \mathrm{m}$ & $37 \mathrm{~T} / \mathrm{m}$ \\
\hline $\begin{array}{l}\text { Magnetostatic } \\
\text { focusing, no } \\
\text { compression }\end{array}$ & constant & $\propto T^{\frac{1}{2}}$ & $\propto T^{\frac{1}{2}}$ & $\propto T^{-\frac{1}{2}}$ & constant & slow \\
\hline$T=200 \mathrm{MeV}$ & $10.6 \mathrm{~m}$ & $1.73 \mathrm{~m}$ & $15.3 \mathrm{~A}$ & 0.127 & $1000 \mathrm{kV} / \mathrm{m}$ & $34.1 \mathrm{~T} / \mathrm{m}$ \\
\hline
\end{tabular}

Table 1.3: Beam parameters of the preliminary IRE design at different locations along the accelerator.

Early design considerations for final focus systems were presented at the first summer study of heavy ion inertial fusion, held at the Claremont Hotel in Berkeley in 1976 [37], [45]. These papers considered idealized focusing system only, and space-charge effects were either ignored or treated in a rudimentary way.

At the Heavy Ion Fusion Workshop held in 1977 at Brookhaven National Laboratory, nonideal effects such as geometric and chromatic aberrations were studied, as well as methods to correct these using sextupole and octupole magnets [15], [18], [44]. Aberrations were treated perturbatively, and nonlinear space-charge effects were ignored.

David Neuffer considered geometric aberrations more generally for the case of zero-current beams in a paper at the Heavy Ion Fusion Workshop held in 1978 at Argonne National Laboratory [77], offering some constraints on heavy ion fusion parameters. Sextupoles and octupoles may be used in the final focus system to compensate for geometric as well as chromatic aberrations [22], [77]. More generally, octupoles can be used to correct 
for geometric and fringe field aberrations simultaneously [48]. The increase in spot size due to chromatic aberrations may be harder to correct with higher-order multipoles [92].

At the Heavy Ion Fusion Workshop at the Claremont Hotel in Berkeley in 1979, a conceptual final focus beam line for a zero-current beam of $10 \mathrm{GeV} \mathrm{U}^{4+}$ was presented, in which the chromatic aberrations are nullified to second order [14]. Third order geometric aberrations were studied for the same beam line by Eugene Colton [23]. The effect of the geometric aberrations was found to be negligible. This beam line, without the sextupole corrections, was then modified to take space charge of the beam into account [36]. The space charge was assumed to be uniformly distributed in configuration space, so that the resulting defocusing force was proportional to the distance from the beam centroid. It was shown though that space-charge nonuniformities can severely affect the beam dynamics [64]. Irving Haber presented the first numerical simulations of space-charge aberrations in final focus systems, using a simple idealized final focusing system [43]. These simulations indicated that space-charge nonlinearities can significantly increase the final spot size, and it was concluded that any extensive final focus design should be accompanied by a program of numerical simulations.

This beamline was subsequently modified to reduce the effects of the space-charge nonlinearities, ignoring the problem of chromatic aberrations [60]. One of the goals of the new design was to eliminate the narrow waists in the beam envelopes before the final focus that existed in the previous design, which seemed to be sensitive to disturbances. The spot size doubled due to the presence of space-charge nonlinearities, which was an improvement over the nine-fold increase in previous designs. 
In the HIBALL-II design, a final focus system is presented for a beam of $10 \mathrm{GeV}$ $\mathrm{Bi}^{+}$ions [35]. Again beam waists are avoided because of the strong space charge of the beam. Instead, a long drift section is used to widen the beam. The beam line is bent to avoid backstreaming neutrons to hit the magnets and cause activation problems, using an achromatic design with two consecutive bending magnets to compensate for dispersion. Calculations of third order geometric aberrations were performed, which included the linear space-charge effect on the particle orbits but not the nonlinear effects due to space-charge nonuniformities. The final focus system was optimized to be free of second and higher order geometric aberrations, but it does have a second order chromatic aberration. Particular attention was given to neutron shielding of the final focus magnets.

An assessment of final focus systems was made in 1987 [12]. In addition, the requirements for a drift compression section upstream of the final focus system were considered. At the end of the accelerator, the beam has a pulse duration of about 100 to $400 \mathrm{~ns}$. The pulse duration is reduced to about 5 to 20 ns by putting a head-to-tail longitudinal velocity tilt on the beam and let it compress in a drift line. The longitudinal space-charge force will reduce the velocity tilt. The aim is to reduce the velocity tilt to zero by the time the beam passes through the final focus system to avoid chromatic aberrations. The velocity tilt may be partly converted into a final momentum spread, which can cause severe chromatic aberrations.

Even if the velocity tilt can be reduced to zero by the time the beam reaches the center of the final focus section, usually the final focus system is long enough for an appreciable velocity tilt to exist both before and after the midpoint. The resulting achromatism 
can be reduced by appropriately using symmetry in the design of the final focus system with respect to its longitudinal midpoint [46].

As supercomputers became more powerful in recent years, more comprehensive simulations of final focus systems are now possible. In particular, the effect of nonlinear space-charge forces in final focus can now be studied more accurately. Also, more comprehensive studies of nonlinear beam dynamics can be performed, in which the main nonideal effects likely to be present in reality can be included in the simulation. Finally, due to the increase in computing power, simulation methods other than particle-in-cell codes, such as Vlasov codes, can be used to validate the simulation results.

This thesis is aimed at gaining a better understanding of drift compression systems and final focus systems for high-current beams, such as those needed for heavy ion inertial fusion. Several drift compression system were designed for the IRE using a new computer code, based on a longitudinal fluid model combined with a transverse envelope model, to accurately calculate the longitudinal electric field. Previous designs of drift compression systems relied on the $g$-factor model, which is insufficiently accurate for high-intensity beams. For the drift compression designs presented in this thesis, particular attention was given to the coupling between longitudinal and transverse dynamics. As the beam compresses, a rapidly increasing current near the beam ends may result in mismatches. These mismatches are undesirable, as they may lead to emittance growth and halo formation. Mismatches produced by longitudinal bunch compression have not been studied sufficiently in the past.

In final focus systems, a beam is usually expanded transversely before it is focused onto a spot. Third-order aberrations due to this expansion may affect the beam quality and 
thereby the achievable spot size. While in the absence of aberrations a smaller spot size can be realized by focusing the beam onto the target using a large convergence angle, the thirdorder aberrations become more severe if the convergence angle is increased. An improvement in the achievable spot size may be obtained by rematching the final focus quadrupoles, which would alleviate the need for additional elements in the final focus system to correct the aberrations. Chapter 5 describes the results of final focus rematching for different values of the convergence angle.

Detailed simulations were made of the Scaled Final Focus Experiment at Lawrence Berkeley National Laboratory. Simulations can be used to assess which nonideal effects in the experiment are likely to significantly affect the beam dynamics. In the simulations of the Scaled Final Focus Experiment, the experimental conditions were replicated accurately, particularly with regards to the presence of conducting surfaces in the experiment. This allows meaningful comparisons between the simulation and the experimental results. In addition, the cause of the beam rotation measured in the experiment was analyzed using a linear model to describe beam and quadrupole rotations. 


\section{Chapter 2}

\section{Charged Particle Beam Dynamics}

\subsection{Beam Dynamics in the Zero-Current Limit}

Consider a beam of particles with charge $q e$ and rest mass $m$ propagating in the positive $z$-direction. The transverse equations of motion are

$$
\begin{aligned}
\frac{\mathrm{d} \vec{p}_{\perp}}{\mathrm{d} t} & =q e(\vec{E}+\vec{v} \times \vec{B})_{\perp}, \\
\frac{\mathrm{d} \vec{r}_{\perp}}{\mathrm{d} t} & =\frac{\vec{p}_{\perp}}{m \gamma},
\end{aligned}
$$

in which $\vec{E}$ and $\vec{B}$ are the electric and magnetic field, $\vec{v} \equiv \vec{\beta} c$ is the velocity, $\gamma \equiv\left(1-\beta^{2}\right)^{-1 / 2}$ is the relativistic factor, and $\vec{r}_{\perp}$ and $\vec{p}_{\perp}$ are the position and momentum transverse to $\hat{z}$. In the absence of acceleration, we can write this as

$$
\frac{\mathrm{d}^{2} \vec{r}_{\perp}}{\mathrm{d} t^{2}}=\frac{q e}{m \gamma}(\vec{E}+\vec{v} \times \vec{B})_{\perp}
$$

The electromagnetic field acting on a particle consists of an externally applied focusing field and the collective beam-particle interaction due to the space-charge repulsion. We 
ignore the latter in the zero-current limit. Since the focusing fields are a function of the longitudinal dimension $z$, it is convenient to express the transverse motion of the particle as a function of $z$ instead of time. Using

$$
\frac{\mathrm{d}}{\mathrm{d} t}=\beta_{z} c \frac{\mathrm{d}}{\mathrm{d} z}
$$

we find

$$
\vec{r}_{\perp}^{\prime \prime}=\frac{q e}{m \gamma \beta_{z}^{2} c^{2}}(\vec{E}+\vec{v} \times \vec{B})_{\perp}
$$

in which the prime denotes differentiation with respect to $z$.

If the transverse velocity is much smaller than the forward velocity, we can apply the paraxial approximation, in which the transverse motion effectively decouples from the longitudinal motion [66]. The paraxial approximation is valid if the focal lengths of external elements are long compared with the beam radius, and the total current $I$ is small compared with the vacuum Alfvén current $I_{\mathrm{A}}$ :

$$
I \ll I_{\mathrm{A}}=\frac{4 \pi \epsilon_{0} m(\beta \gamma c)^{3}}{q e} .
$$

In the case of a $2.5 \mathrm{kA}$ beam of $\mathrm{Cs}^{+}$ions at a velocity $\beta \approx 1 / 3$, which are typical parameters for a heavy-ion inertial fusion driver, the Alfvén current is 183 MA. So the paraxial approximation will be quite good in the present case. In this approximation, we can replace $\beta_{z} c$ by the total speed $\beta c$. The equation of motion then becomes

$$
\vec{r}_{\perp}^{\prime \prime}=\frac{q e}{m \gamma \beta^{2} c^{2}}\left(\vec{E}_{\perp}+\beta c \hat{z} \times \vec{B}\right)
$$

If we include acceleration, this equation becomes

$$
\vec{r}_{\perp}^{\prime \prime}+\frac{(\beta \gamma)^{\prime}}{\beta \gamma} \vec{r}_{\perp}^{\prime}=\frac{q e}{m \gamma \beta^{2} c^{2}}\left(\vec{E}_{\perp}+\beta c \hat{z} \times \vec{B}\right)
$$


Next, we consider the transverse focusing fields. An electrostatic or magnetostatic field in a source-free region can be expanded in terms of its multipole components [42]:

$$
\begin{aligned}
E_{r} & =-\sum_{\nu=0}^{\infty} \frac{1}{2(\nu+1)} \frac{\mathrm{d} e_{0, \nu}(z)}{\mathrm{d} z} r^{2 \nu+1} \\
& +\sum_{n=1}^{\infty} \sum_{\nu=0}^{\infty} e_{n, \nu}(z)\left(1+\frac{2 \nu}{n}\right) r^{n-1+2 \nu} \cos \left[n\left(\theta+\phi_{n}\right)\right] \\
E_{\theta} & =-\sum_{n=1}^{\infty} \sum_{\nu=0}^{\infty} e_{n, \nu}(z) r^{n-1+2 \nu} \sin \left[n\left(\theta+\phi_{n}\right)\right] \\
E_{z} & =\sum_{\nu=0}^{\infty} e_{0, \nu}(z) r^{2 \nu}+\sum_{n=1}^{\infty} \sum_{\nu=0}^{\infty} \frac{1}{n} \frac{\mathrm{d} e_{n, \nu}(z)}{\mathrm{d} z} r^{n+2 \nu} \cos \left[n\left(\theta+\phi_{n}\right)\right] .
\end{aligned}
$$

The magnetic field can be decomposed similarly:

$$
\begin{aligned}
B_{r} & =-\sum_{\nu=0}^{\infty} \frac{1}{2(\nu+1)} \frac{\mathrm{d} b_{0, \nu}(z)}{\mathrm{d} z} r^{2 \nu+1} \\
& +\sum_{n=1}^{\infty} \sum_{\nu=0}^{\infty} b_{n, \nu}(z)\left(1+\frac{2 \nu}{n}\right) r^{n-1+2 \nu} \sin \left[n\left(\theta+\phi_{n}\right)\right] \\
B_{\theta} & =\sum_{n=1}^{\infty} \sum_{\nu=0}^{\infty} b_{n, \nu}(z) r^{n-1+2 \nu} \cos \left[n\left(\theta+\phi_{n}\right)\right] \\
B_{z} & =\sum_{\nu=0}^{\infty} b_{0, \nu}(z) r^{2 \nu}+\sum_{n=1}^{\infty} \sum_{\nu=0}^{\infty} \frac{1}{n} \frac{\mathrm{d} b_{n, \nu}(z)}{\mathrm{d} z} r^{n+2 \nu} \sin \left[n\left(\theta+\phi_{n}\right)\right]
\end{aligned}
$$

For these fields to obey Maxwell's equations, the multipole components $e_{n, \nu}$ need to satisfy the recursion relation:

$$
e_{n, \nu+1}(z)=-\frac{1}{4(\nu+1)(n+\nu+1)} \frac{\mathrm{d}^{2} e_{n, \nu}(z)}{\mathrm{d} z^{2}}
$$

due to Faraday's law for static fields $\vec{\nabla} \times \vec{E}=0$, and similarly for the magnetic multipole components $b_{n, \nu}(z)$ due to Ampère's law for static fields $\vec{\nabla} \times \vec{B}=0$ in a source-free region. Multipole components with different $n$ are independent of each other.

Alternating-gradient accelerators rely on electrostatic or magnetic quadrupoles $(n=2, \nu=0)$ to focus the beam. The transverse electrostatic quadrupole field is given in 
terms of the Cartesian coordinates $x=r \cos \theta, y=r \sin \theta$ as

$$
\begin{aligned}
\vec{E}_{\perp} & =e_{2,0}(z) r\left[\hat{r} \cos \left(2\left(\theta-\phi_{2}\right)\right)-\hat{\theta} \sin \left(2\left(\theta-\phi_{2}\right)\right)\right] \\
& =G_{E}(z)(\hat{x} x-\hat{y} y)+S_{E}(z)(\hat{x} y+\hat{y} x)
\end{aligned}
$$

in which $G_{E} \equiv e_{2,0}(z) \cos \left(2 \phi_{2}\right)$ and $S_{E} \equiv e_{2,0}(z) \sin \left(2 \phi_{2}\right)$. The second term in this equation represents a skew quadrupole, which couples the horizontal $(x)$ and vertical $(y)$ motion. For this reason, skew quadrupoles are generally avoided in the design of accelerators. For positive field gradient $G_{E}$, the first term in equation (2.17) gives a focusing force in the horizontal $(x)$ direction and a defocusing force in the vertical $(y)$ direction:

$$
\begin{aligned}
x^{\prime \prime} & =-\frac{q e}{m \gamma \beta^{2} c^{2}} G_{E} x, \\
y^{\prime \prime} & =+\frac{q e}{m \gamma \beta^{2} c^{2}} G_{E} y .
\end{aligned}
$$

For magnetostatic fields, we find similarly

$$
\begin{aligned}
\vec{B}_{\perp} & =b_{2,0}(z) r\left[\hat{r} \sin \left(2\left(\theta-\phi_{2}\right)\right)+\hat{\theta} \cos \left(2\left(\theta-\phi_{2}\right)\right)\right] \\
& =G_{B}(z)(\hat{x} y+\hat{y} x)+S_{B}(z)(\hat{x} x-\hat{y} y)
\end{aligned}
$$

in which $G_{B}(z) \equiv b_{2,0}(z) \cos \left(2 \phi_{2}\right)$ and $S_{B}(z) \equiv b_{2,0}(z) \sin \left(2 \phi_{2}\right)$. Again the term proportional to $S_{B}(z)$ represents a skew quadrupole. For positive field gradient $G_{B}$, the first term in equation (2.20) gives a defocusing force in the horizontal $(x)$ direction and a focusing force in the vertical $(y)$ direction:

$$
\begin{aligned}
& x^{\prime \prime}=+\frac{q e}{m \gamma \beta c} G_{B} x, \\
& y^{\prime \prime}=-\frac{q e}{m \gamma \beta c} G_{B} y .
\end{aligned}
$$


We can then write the equation of motion of a single charged particle as

$$
\begin{aligned}
& x^{\prime \prime}+k_{x}(z) x=0, \\
& y^{\prime \prime}+k_{y}(z) y=0,
\end{aligned}
$$

in which $k_{\bullet}(z)$ is determined by the electrostatic and magnetostatic quadrupole fields described above. Usually in a transport section, $k_{\bullet}(z)$ has zero average and is periodic with period $2 L$, in which $L$ is the lattice half period. In this thesis, a lattice half period is defined as a distance $L$ containing one quadrupole centered around $\frac{1}{2} L$. This definition is convenient in the design of a drift compression system as described in chapter 4. In transport lattices though, doublets are commonly used, in which quadrupoles are not centered around $\frac{1}{2} L$.

A beam particle in a transverse focusing force as in described by equations (2.22), (2.23) experiences an alternating sequence of focusing and defocusing forces. Since the particle orbits in such an alternating-gradient lattice deviate farther from the axis in focusing lenses than in defocusing lenses, the particles experience a focusing force on average.

The $z$-dependence of the quadrupole field results in non-zero second derivatives in the recursion equation (2.15), in particular near the ends of the focusing elements. This gives rise to higher-order multipoles (called pseudo-multipoles) in the fringe fields of focusing elements. We will ignore this effect for now, but we will consider it in section 2.4.1.

\subsection{Beam Dynamics in the Presence of Space Charge}

In the zero-current limit, all particles in the beam move independently of each other. To self-consistently include the beam self-repulsion due to its space charge, we need to consider the evolution of the beam distribution in the transverse $x, x^{\prime}, y, y^{\prime}$ phase space. 
The Kapchinskij-Vladimirskij (K-V) distribution is the only repeating distribution presently known that self-consistently describes beam propagation in an alternatinggradient focusing lattice [55]. Mathematically, the K-V distribution is described by a deltafunction in four-dimensional transverse phase space:

$$
f\left(x, x^{\prime}, y, y^{\prime}\right)=\frac{N}{\pi \varepsilon_{x} \cdot \pi \varepsilon_{y}} \delta\left[\frac{a^{2}}{\varepsilon_{x}^{2}}\left(x^{\prime}-x \frac{a^{\prime}}{a}\right)^{2}+\frac{x^{2}}{a^{2}}+\frac{b^{2}}{\varepsilon_{y}^{2}}\left(y^{\prime}-y \frac{b^{\prime}}{b}\right)^{2}+\frac{y^{2}}{b^{2}}-1\right]
$$

in which $N$ is the number of particles per unit length along the beam. Note that in this expression, the parameters $a, a^{\prime}, b$, and $b^{\prime}$ depend on the longitudinal coordinate $z$.

To explain the parameters appearing in this expression, we will calculate several moments of the K-V distribution. First we integrate equation (2.24) over the primed variables. This yields the particle distribution in transverse configuration space

$$
n(x, y)=\int f\left(x, x^{\prime}, y, y^{\prime}\right) \mathrm{d} x^{\prime} \mathrm{d} y^{\prime}=\frac{N}{\pi a b} \theta\left(1-\frac{x^{2}}{a^{2}}-\frac{y^{2}}{b^{2}}\right)
$$

in which $\theta$ is the Heaviside step function. This represents a uniform distribution over an ellipse with semi-axes $a$ and $b$. From this equation, we find

$$
\begin{aligned}
& a^{2}=4\left\langle x^{2}\right\rangle, \\
& b^{2}=4\left\langle y^{2}\right\rangle,
\end{aligned}
$$

in which the average $\langle\cdot\rangle$ is defined by

$$
\left\langle g\left(x, x^{\prime}, y, y^{\prime}\right)\right\rangle \equiv \frac{1}{N} \int f\left(x, x^{\prime}, y, y^{\prime}\right) g\left(x, x^{\prime}, y, y^{\prime}\right) \mathrm{d} x \mathrm{~d} x^{\prime} \mathrm{d} y \mathrm{~d} y^{\prime}
$$

Taking the derivative with respect to $z$ of equations (2.26) and (2.27) and evaluating the resulting moments using (2.28) yields

$$
a \frac{\mathrm{d} a}{\mathrm{~d} z}=4\left\langle x x^{\prime}\right\rangle=a a^{\prime},
$$




$$
b \frac{\mathrm{d} b}{\mathrm{~d} z}=4\left\langle y y^{\prime}\right\rangle=b b^{\prime}
$$

So we found that $a^{\prime}=\frac{\mathrm{d} a}{\mathrm{~d} z}$ and $b^{\prime}=\frac{\mathrm{d} b}{\mathrm{~d} z}$.

The space-charge field inside an ellipse with a uniform space-charge distribution is given by

$$
\begin{aligned}
\vec{E} & =\frac{\lambda}{\pi \epsilon_{0}} \frac{1}{a+b}\left(\frac{x \hat{x}}{a}+\frac{y \hat{y}}{b}\right) \\
\vec{B} & =\frac{\mu_{0} \lambda}{\pi} \beta c \frac{1}{a+b}\left(-\hat{x} \frac{y}{b}+\hat{y} \frac{x}{a}\right)
\end{aligned}
$$

in which $\lambda$ is the line charge density of the beam [62]. Substituting these fields into equation (2.7) yields

$$
x^{\prime \prime}=\frac{q e}{m} \frac{2 I}{4 \pi \epsilon_{0}(\beta \gamma c)^{3}} \frac{2}{a+b} \frac{x}{a},
$$

in which $I=\lambda \beta c$ is the beam current. Perveance is defined as a dimensionless measure of the magnitude of the space-charge repulsion:

$$
Q \equiv \frac{q e}{m} \frac{2 I}{4 \pi \epsilon_{0}(\beta \gamma c)^{3}}=\frac{2 I}{I_{\mathrm{A}}}
$$

The perveance is typically between $10^{-5}$ and $10^{-3}$ in the final focus section of a driver for heavy ion inertial fusion.

For the full equation of motion in the transverse direction, we need to combine the space-charge repulsion and the external focusing force. We find from equations $(2.22)$ and (2.33):

$$
x^{\prime \prime}+k_{x}(z) x=\frac{2 Q}{(a+b)} \frac{x}{a},
$$

and similarly in the $y$-direction

$$
y^{\prime \prime}+k_{y}(z) y=\frac{2 Q}{(a+b)} \frac{y}{b} .
$$


To find the envelope equation, which describes the evolution of the beam radii as a function of $z$, we take the derivative of equation (2.29) with respect to $z$ :

$$
a^{\prime 2}+a a^{\prime \prime}=4\left\langle x^{2}\right\rangle+4\left\langle x x^{\prime \prime}\right\rangle
$$

In this equation, we can substitute $a^{\prime}$ from equation (2.29):

$$
a^{\prime \prime}=\frac{4\left\langle x^{2}\right\rangle+4\left\langle x x^{\prime \prime}\right\rangle}{a}-\frac{16\left\langle x x^{\prime}\right\rangle^{2}}{a^{3}}
$$

Next we substitute $x^{\prime \prime}$ from equation (2.35) and then $\left\langle x^{2}\right\rangle$ from equation (2.26) to find

$$
a^{\prime \prime}+k_{x}(z) a=16 \frac{\left\langle x^{\prime 2}\right\rangle\left\langle x^{2}\right\rangle-\left\langle x x^{\prime}\right\rangle^{2}}{a^{3}}+\frac{2 Q}{a+b}
$$

The numerator of the first term on the right-hand-side can be calculated from the $\mathrm{K}-\mathrm{V}$ distribution (2.24). We find

$$
16\left(\left\langle x^{2}\right\rangle\left\langle x^{\prime 2}\right\rangle-\left\langle x x^{\prime}\right\rangle^{2}\right)=\varepsilon_{x}^{2}
$$

To understand the physical significance of $\varepsilon_{x}$, we integrate the K-V distribution over $y$ and $y^{\prime}$. This yields

$$
\int f\left(x, x^{\prime}, y, y^{\prime}\right) \mathrm{d} y \mathrm{~d} y^{\prime}=\frac{N}{\pi \varepsilon_{x}} \theta\left(1-\frac{x^{2}}{a^{2}}-\frac{a^{2}}{\varepsilon_{x}^{2}}\left(x^{\prime}-x \frac{a^{\prime}}{a}\right)^{2}\right) .
$$

This represents a uniformly filled ellipse in $x, x^{\prime}$ phase space with area $\pi \varepsilon_{x}$. As $a$ and $a^{\prime}$ evolve during propagation in an alternating-gradient focusing channel, the ellipse changes its ellipticity and rotates in $x, x^{\prime}$ phase space.

The emittance of a beam is defined as the area, in units of $\pi$, of an ellipse in phase space enclosing the area occupied by the beam. In the horizontal direction, it is therefore equal to $\varepsilon_{x}$ in the expression for a $\mathrm{K}-\mathrm{V}$ distribution (2.24). The emittance in 
terms of second-order moments over the beam distribution (2.40) is often referred to as the $4 \times$ RMS emittance, or as the edge emittance. For a $\mathrm{K}-\mathrm{V}$ beam, these two definitions for the emittance are equivalent.

We can demonstrate that the emittance of a coasting $\mathrm{K}-\mathrm{V}$ beam is preserved by calculating the derivative with respect to $z$ of the $4 \times$ RMS emittance:

$$
\left(\varepsilon_{x}^{2}\right)^{\prime}=32\left(\left\langle x^{\prime} x^{\prime \prime}\right\rangle\left\langle x^{2}\right\rangle-\left\langle x x^{\prime \prime}\right\rangle\left\langle x x^{\prime}\right\rangle\right)
$$

Substituting $x^{\prime \prime}$ from equation (2.35) now gives

$$
\left(\varepsilon_{x}^{2}\right)^{\prime}=32\left(-k_{x}(z)+\frac{2 Q}{(a+b) a}\right)\left(\left\langle x^{\prime} x\right\rangle\left\langle x^{2}\right\rangle-\left\langle x^{2}\right\rangle\left\langle x x^{\prime}\right\rangle\right)=0 .
$$

Note that linearity of the transverse force is required for $\left(\varepsilon_{x}^{2}\right)^{\prime}$ to vanish. Field nonlinearities may lead to phase space filamentation and dilution, and therefore to a larger effective emittance [27]. While the $4 \times$ RMS emittance may increase in the presence of nonlinear fields, the true area in phase space actually occupied by the beam is conserved, as required by Liouville's theorem [38]. In the presence of acceleration, the normalized emittance $\beta \gamma \varepsilon_{x}$ is conserved in linear systems instead of the unnormalized emittance $\varepsilon_{x}$.

In terms of the emittance, the envelope equation can now be written as

$$
a^{\prime \prime}+k_{x}(z) a=\frac{\varepsilon_{x}^{2}}{a^{3}}+\frac{2 Q}{a+b} .
$$

For the $y$-direction we find a similar equation:

$$
b^{\prime \prime}+k_{y}(z) b=\frac{\varepsilon_{y}^{2}}{b^{3}}+\frac{2 Q}{a+b} .
$$

Note that these equations are coupled through their dependence on the perveance $Q$. 
Now we can show that the $\mathrm{K}-\mathrm{V}$ distribution satisfies the transverse Vlasov equation

$$
\frac{\mathrm{d} f}{\mathrm{~d} z}=\frac{\partial f}{\partial z}+\frac{\partial f}{\partial x} x^{\prime}+\frac{\partial f}{\partial x^{\prime}} x^{\prime \prime}+\frac{\partial f}{\partial y} y^{\prime}+\frac{\partial f}{\partial y^{\prime}} y^{\prime \prime}=0
$$

Consider the Courant-Snyder invariants

$$
\begin{aligned}
I_{x} & \equiv \frac{a^{2}}{\varepsilon_{x}^{2}}\left(x^{\prime}-x \frac{a^{\prime}}{a}\right)^{2}+\frac{x^{2}}{a^{2}} \\
I_{y} & \equiv \frac{b^{2}}{\varepsilon_{y}^{2}}\left(y^{\prime}-y \frac{b^{\prime}}{b}\right)^{2}+\frac{y^{2}}{b^{2}}
\end{aligned}
$$

which are conserved along particle orbits:

$$
I_{x}^{\prime}=2\left(\frac{a^{2}}{\varepsilon_{x}^{2}}\left(a x^{\prime \prime}-a^{\prime \prime} x\right)+\frac{x}{a}\right)\left(\frac{a x^{\prime}-a^{\prime} x}{a^{2}}\right)=0
$$

upon substitution of equations (2.35) and (2.44), and similarly for $I_{y}$. Since the K-V distribution can then by written as $f\left(x, x^{\prime}, y, y^{\prime}\right)=\frac{N}{\pi \varepsilon_{x} \cdot \pi \varepsilon_{y}} \delta\left[I_{x}+I_{y}-1\right]$, it follows from Jeans' theorem that the K-V distribution solves the Vlasov equation [56], [78].

The $\mathrm{K}-\mathrm{V}$ distribution is of importance in the theory of beam dynamics as an ideal case, in which the space-charge field is guaranteed to be linear. Due to the highly singular nature of a delta-function in four-dimensional phase space, a $\mathrm{K}-\mathrm{V}$ distribution cannot be expected to accurately reflect the distribution of real beams. In addition, it can be shown that the K-V distribution is unstable [91]. The equations (2.26), (2.27), (2.29), (2.30), and (2.40) are strictly speaking only valid for the K-V distribution. Nevertheless, they are commonly used in experiments and simulations to evaluate the beam parameters $a, b, a^{\prime}$, $b^{\prime}$ and the emittance in terms of moments over the beam distribution.

In simulations, instead of a $\mathrm{K}-\mathrm{V}$ distribution often a semi-Gaussian distribution is used as the initial particle distribution. This distribution is characterized by a uniformly 
filled ellipse in configuration space, while the velocities $x^{\prime}, y^{\prime}$ have a Maxwellian distribution.

We can write this distribution as

$$
\begin{aligned}
f\left(x, x^{\prime}, y, y^{\prime}\right)= & \frac{4}{\pi} \theta\left(1-\frac{x^{2}}{a^{2}}-\frac{y^{2}}{b^{2}}\right) \frac{1}{2 \pi \varepsilon_{x} \varepsilon_{y}} \\
& \exp \left[-\frac{1}{2}\left(\frac{2 a}{\varepsilon_{x}}\right)^{2}\left(x^{\prime}-\frac{x}{a} a^{\prime}\right)^{2}-\frac{1}{2}\left(\frac{2 b}{\varepsilon_{y}}\right)^{2}\left(y^{\prime}-\frac{y}{b} b^{\prime}\right)^{2}\right] .
\end{aligned}
$$

Unlike the $\mathrm{K}-\mathrm{V}$ distribution, a semi-Gaussian beam does not remain semi-Gaussian as it propagates in an alternating-gradient lattice.

\subsection{Beam Dynamics in the Smooth Approximation}

The equation of motion for a single particle (2.35), (2.36) in a focusing lattice has a cosine-like $\left(x^{\prime}(z=0)=0\right)$ and a sine-like $(x(z=0)=0)$ trajectory as its two independent solutions. Each of these trajectories consist of a fast oscillation at a frequency corresponding to the period of the focusing lattice, as well as a slower oscillation extending over several lattice periods. Usually we are mostly interested in this smooth, slower oscillatory behavior of the particle, which is called the betatron oscillation [27]. Effectively, this means that we approximate the cosine- and sine-like trajectories as cosines and sines, which follow the smooth equation of motion

$$
x_{0}^{\prime \prime}(z)+\left(\frac{\sigma_{0}}{2 L}\right)^{2} x_{0}(z)=0
$$

in which $x_{0}(z)$ represents the smooth orbit of the particle and $\sigma_{0}$ is the (undepressed) tune or the phase advance per period. The undepressed tune can be calculated exactly from the properties of the focusing lattice using the transfer matrix approach [27]. 
In the presence of space charge, the smooth equation of motion becomes

$$
\begin{aligned}
x_{0}^{\prime \prime}+\left(\frac{\sigma_{0}}{2 L}\right)^{2} x_{0} & =\frac{2 Q}{\left(a_{0}+b_{0}\right) a_{0}} x_{0}, \\
y_{0}^{\prime \prime}+\left(\frac{\sigma_{0}}{2 L}\right)^{2} y_{0} & =\frac{2 Q}{\left(a_{0}+b_{0}\right) b_{0}} y_{0}
\end{aligned}
$$

in which $a_{0}(z)$ and $b_{0}(z)$ are the smooth beam envelopes given by

$$
\begin{aligned}
a_{0}^{\prime \prime}+\left(\frac{\sigma_{0}}{2 L}\right)^{2} a_{0} & =\frac{\varepsilon_{x}^{2}}{a_{0}^{3}}+\frac{2 Q}{a_{0}+b_{0}}, \\
b_{0}^{\prime \prime}+\left(\frac{\sigma_{0}}{2 L}\right)^{2} b_{0} & =\frac{\varepsilon_{y}^{2}}{b_{0}^{3}}+\frac{2 Q}{a_{0}+b_{0}} .
\end{aligned}
$$

From the smooth envelope equations, we can find an expression for the average beam radius:

$$
\left(\frac{\sigma_{0}}{2 L}\right)^{2} R=\frac{\varepsilon^{2}}{R^{3}}+\frac{Q}{R}
$$

Defining the depressed tune $\sigma$ by

$$
\left(\frac{\sigma}{2 L}\right)^{2} \equiv\left(\frac{\sigma_{0}}{2 L}\right)^{2}-\frac{Q}{R^{2}}
$$

we can rewrite this as

$$
\left(\frac{\sigma}{2 L}\right)^{2} R=\frac{\varepsilon^{2}}{R^{3}}
$$

We define the betatron tune depression [82] as the ratio $\sigma / \sigma_{0}$.

The envelope equation for a round beam after the last final focus magnet, converging onto a spot on the target is given by

$$
R^{\prime \prime}=\frac{Q}{R}+\frac{\varepsilon^{2}}{R^{3}}
$$

When the beam approaches the target, the beam radius is small and the emittance term dominates over the perveance term. So although for space-charge dominated beams the emittance is negligible in most of the accelerator, the magnitude of the emittance determines the achievable spot size. 


\subsubsection{Approximate Envelope Relations}

Some approximate relations can be derived between the depressed and undepressed tune, and the lattice and beam properties, by employing expansions in $k L^{2}$, in which $k$ is the focusing strength of the quadrupole and $L$ is the lattice half period [67]. We will make extensive use of two of these relations when designing the IRE drift compression and final focus systems. The first equation relates the average beam radius $\bar{a}$ to the perveance $Q$, the lattice half period $L$, and the depressed and undepressed tunes $\sigma$ and $\sigma_{0}$ :

$$
Q\left(\frac{2 L}{\bar{a}}\right)^{2} \approx 2\left(\cos \sigma-\cos \sigma_{0}\right)
$$

Since the depressed tune $\sigma$ is small for space-charge dominated beams, usually we can set $\cos \sigma=1$ in this equation.

The occupancy $\eta$ of a lattice half period is defined as the fraction of its length that is occupied by the quadrupole. For a transverse focusing lattice with flat-top quadrupole fields, we can find the undepressed tune from

$$
\cos \sigma_{0} \approx 1-\frac{1}{2}\left(\eta k L^{2}\right)^{2}\left(1-\frac{2}{3} \eta\right)
$$

These equations typically have an accuracy of $1 \%$ or better.

\subsection{Nonlinear Effects}

In this section, some of the nonideal effects that are present in actual accelerators are described. Nonlinear forces acting on a beam tend to increase its RMS emittance and thereby the achievable spot size. They can often only be studied perturbatively or with the 
aid of computer simulations. Some of the nonlinear effects mentioned here can be mitigated by appropriately designing the accelerator.

\subsubsection{Third Order Aberrations}

\section{Geometric Aberrations}

In our derivation of the single particle equation of motion (2.22), we made use of the paraxial approximation, in which the transverse particle velocity is ordered small in comparison to the longitudinal velocity. When the transverse velocity is taken into account, third-order nonlinear terms appear in the equation of motion.

We start from the equation of motion (2.3) for a single particle with time as the independent variable. In the presence of magnetic fields only, the magnitude of the momentum of each particle is conserved:

$$
\frac{\mathrm{d}}{\mathrm{d} t}(m \gamma v)=0
$$

We can write the velocity $v$ in terms of the longitudinal velocity $v_{z}$ as

$$
v=v_{z} \sqrt{1+x^{\prime 2}+y^{\prime 2}}
$$

From this we find that

$$
\begin{aligned}
\frac{\mathrm{d}^{2}}{\mathrm{~d} t^{2}} & =v_{z} \frac{\mathrm{d}}{\mathrm{d} z} v_{z} \frac{\mathrm{d}}{\mathrm{d} z} \\
& =\frac{(\beta c)^{2}}{1-x^{\prime 2}-y^{\prime 2}}\left(\frac{\mathrm{d}^{2}}{\mathrm{~d} z^{2}}-\frac{x^{\prime} x^{\prime \prime}+y^{\prime} y^{\prime \prime}}{1-x^{\prime 2}-y^{\prime 2}} \frac{\mathrm{d}}{\mathrm{d} z}\right)
\end{aligned}
$$

Substituting this into the equation of motion (2.3), we find two coupled nonlinear differential equations:

$$
\left(1+y^{\prime 2}\right) x^{\prime \prime}-x^{\prime} y^{\prime} y^{\prime \prime}=\frac{q e}{m \gamma \beta c}\left(1+x^{2}+y^{\prime 2}\right)^{3 / 2}\left(-B_{y}+B_{z} y^{\prime}\right)
$$




$$
\left(1+x^{\prime 2}\right) y^{\prime \prime}-x^{\prime} y^{\prime} x^{\prime \prime}=\frac{q e}{m \gamma \beta c}\left(1+x^{\prime 2}+y^{\prime 2}\right)^{3 / 2}\left(B_{x}-B_{z} x^{\prime}\right)
$$

These equations reduce to the linear equation of motion (2.7) in the paraxial approximation, in which we use $x^{\prime} \ll 1, y^{\prime} \ll 1$. The nonlinear terms in this equation may cause an emittance increase by distorting the occupied transverse phase space and thereby adversely affecting the beam quality.

Simulation codes such as WARP typically solve the $3 \mathrm{~d}$ equation of motion as a function of time instead of longitudinal distance. Even in $2 \mathrm{~d}$ transverse slice calculations, in which all particles are assumed to be at the same longitudinal position at a given time step, the position and velocity of each particle is calculated in all three directions. The geometric aberrations are then included automatically via a changing longitudinal velocity $v_{z}$, instead of explicit terms in the transverse equation of motion.

\section{Fringe Fields}

The fringe fields near the ends of the focusing elements also generate third-order aberrations, arising from the longitudinal variation of the quadrupolar field. We will discuss the effects of fringe fields in case of magnetic quadrupole focusing; the case of electrostatic focusing can be treated analogously.

The term proportional to $b_{2,0}(z)$ in the multipole expansion of the applied magnetic field corresponds to the quadrupolar focusing field $G_{B}(z)=b_{2,0}(z) \cos \left(2 \phi_{2}\right)$ that appears in the equation of motion (2.21). From equation (2.14), there will be a longitudinal field due to the non-zero $z$-derivative of $G_{B}(z)$, which will affect the transverse motion of the 
beam ions. This field is given by

$$
\begin{aligned}
B_{z} & =\frac{1}{2} \frac{\mathrm{d} b_{2,0}(z)}{\mathrm{d} z} r^{2} \sin (2 \theta) \\
& =G_{B}^{\prime}(z) x y .
\end{aligned}
$$

In addition, according to the recursion relation (2.15), the $z$-dependence of $b_{2,0}(z)$ will generate an infinite number of higher-order multipoles in the transverse magnetic field. In the absence of skew quadrupoles, the pseudo-octupole is the first higher-order multipole that appears. Its transverse magnetic field is given by

$$
\begin{aligned}
\vec{B}_{\perp} & =b_{2,1}(z) r^{3}(2 \sin (2 \theta) \hat{r}+\cos (2 \theta) \hat{\theta}) \\
& =-\frac{1}{12} G_{B}^{\prime \prime}(z)\left(\hat{x}\left(3 x^{2} y+y^{3}\right)+\hat{y}\left(3 x y^{2}+x^{3}\right)\right),
\end{aligned}
$$

in which $b_{2,1}(z)=-\frac{1}{12} G_{B}^{\prime \prime}(z)$, where $G_{B}(z)$ is the field gradient of the quadrupole field, follows from the recursion relation (2.15).

If we take these fields into account, third-order terms appear in the equation of motion (2.22), (2.23):

$$
\begin{aligned}
& x^{\prime \prime}=-G_{B}(z) x+G_{B}^{\prime}(z) x y y^{\prime}+G_{B}^{\prime \prime}(z)\left(\frac{x y^{2}}{4}+\frac{x^{3}}{12}\right), \\
& y^{\prime \prime}=+G_{B}(z) y-G_{B}^{\prime}(z) x y x^{\prime}-G_{B}^{\prime \prime}(z)\left(\frac{x^{2} y}{4}+\frac{y^{3}}{12}\right),
\end{aligned}
$$

in addition to the third-order terms due to the geometric aberrations given in equations (2.66), (2.67). A general treatment of third-order aberrations of quadrupole magnet lattices has been given by Meads [73].

To calculate the longitudinal field and the higher-order multipoles, the shape of the fringe field and all its derivatives need to be known. Since a multipole of order $n$ generates 


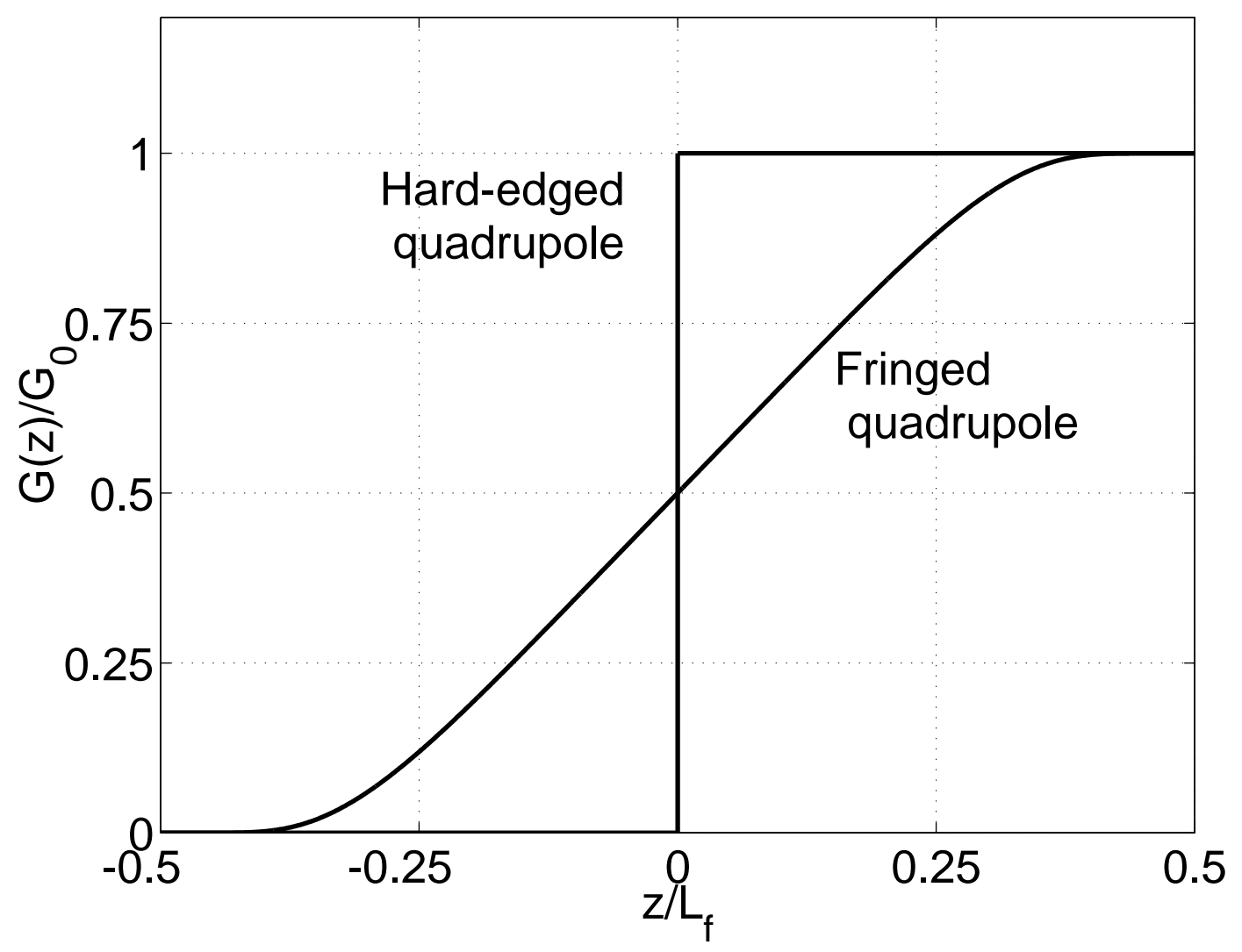

Figure 2.1: The quadrupole field gradient $G(z)$ as a function of distance $z$ in the fringe field of a quadrupole if the fringe field is described by equation (2.73). The length of the fringe field is $L_{\mathrm{f}}$.

terms of order $\left(r_{\text {beam }} / r_{\text {pipe }}\right)^{n-1}$ in the equation of motion, usually only the lowest multipoles are taken into account. The shape of the fringe field can be approximated by some smooth function such as

$$
G(z)=G_{0} \frac{1}{2}\left[1+\tanh \left(\tan \left(\frac{\pi z}{L_{\mathrm{f}}}\right)\right)\right]
$$

in which $G_{0}$ is the quadrupole field gradient in the center of the quadrupole, outside of the fringe field region, and $L_{\mathrm{f}}$ is the length of the fringe field. This function has the property that all its derivatives vanish at $z= \pm \frac{1}{2} L_{\mathrm{f}}$. It is plotted in figure 2.1. 


\subsubsection{Chromatic Aberrations}

So far, we assumed that all beam ions have exactly the same longitudinal velocity. This will not be true in practice. Since the focal strength is proportional to $1 / \beta$ for magnetic quadrupoles, and $1 / \beta^{2}$ for electrostatic quadrupoles, the beam particles will then each experience a different focusing force due to the differences in their longitudinal velocity. These chromatic aberrations can cause a larger spot size in final focus, as well as mismatches and subsequent emittance increase due to phase mixing during acceleration.

The spread in longitudinal velocity originates at the ion source, which has a nonzero temperature, causing the initial velocity of the ions to have a thermal spread. Longitudinal compression as well as nonideal effects in the beam or the accelerator can cause this velocity spread to increase as the ions propagate through the system.

First-order chromatic aberrations are generated by bends, which are not treated in this thesis. Second-order chromatic aberrations can be corrected with the use of sextupole magnets, combined suitably with bends.

\section{Longitudinal Velocity Tilt}

Chromatic aberrations can arise particularly as a consequence of the longitudinal velocity tilt on a beam as it is being compressed. Contemporary designs for fusion power plants as well as for the Integrated Research Experiment, discussed in chapter 1, rely on longitudinal bunch compression by factors up to 100 in the final stages of the machine. In order to compress the beam, the tail of the beam has to travel faster than the head. Typically the velocity tilt is a couple of percent of the mean beam velocity. This velocity 
tilt is much larger than the thermal spread of the beam. However, in contrast to the random thermal velocity spread, the effect of the remaining velocity tilt can be compensated for by adding small time-dependent focusing elements to the final focus system, whose focusing fields are timed such that all parts of the beam can experience the same effective focusing force. In addition, the velocity tilt will eventually be removed by the longitudinal spacecharge repulsion in a charged particle beam. Ideally, the tilt is reduced to zero by the time the beam reaches the center of the final focus system to avoid chromatic aberrations.

\subsubsection{Image Charges}

A charged particle beam will be surrounded by conducting surfaces as it propagates in an accelerator. In case of magnetic focusing, the electric wires of the magnet or the vacuum pipe will form a conducting surface, while in case of electrostatic focusing, the electrodes are conducting. The presence of the charged particle beam will generate image charges on these surfaces such that the electric fields in these conductors are zero. Especially when the beam is close to one of these conductors, the image forces can significantly affect the beam dynamics. The field generated by these image charges will in general contain higher-order multipole fields, which act nonlinearly on the beam.

\subsubsection{Higher Multipole Fields}

In practice, an electrostatic or magnetic quadrupole will also contain higher-order multipoles. They will be absent only if the charges (in case of an electrostatic quadrupole) or the currents (in case of a magnetic quadrupole) on the focusing element have the exact $\cos (2 \theta)$ distribution that is characteristic for quadrupoles. For a focusing element with 
quadrupolar symmetry, only multipoles with the same symmetry can occur. So the lowest possible multipole is the dodecapole $(\cos (6 \theta))$, in which the field depends on the distance from the optical axis as $r^{5}$. Except for beam particles close to the magnet edge, the effect of higher-order multipoles will be small in a well designed and manufactured element.

\subsubsection{Misalignments}

So far, we assumed that both the beam and the focusing elements are perfectly aligned. If a focusing element is not perfectly aligned, the beam experiences a dipole field as it passes through, and will be kicked transversely. The beam centroid motion will then resemble a single particle oscillation in the accelerator. As the beam passes through a number of displaced quadrupoles, its oscillation amplitude will increase on the average, although this increase is mitigated by acceleration. Even if all quadrupoles are perfectly

aligned, an initial beam misalignment will also cause a beam centroid oscillation. The effects of beam misalignments have been investigated for the case of the Integrated Research Experiment [21].

\subsubsection{Beam Distribution}

A particular concern for high-perveance beams is the effect of nonlinear spacecharge forces. So far, we assumed that the beam charge distribution in configuration space is uniform, such that the space-charge force is linear. The charge distribution remains uniform as the beam propagates only in case of a $\mathrm{K}-\mathrm{V}$ distribution, which is a highly idealized distribution function. In reality, the beam distribution will be far from a $\mathrm{K}-\mathrm{V}$ distribution, and nonlinear space-charge forces due to a nonuniform charge distribution will 
appear. Space charge nonlinearities may severely affect the beam, and detailed particle-incell simulations are required to analyze their effect.

\subsubsection{Three-Dimensional Effects}

In two-dimensional transverse slice simulations, the beam is assumed to be infinitely long and all beam particles are assumed to remain in the same transverse slice, such that Poisson's equation needs to be solved in a $2 \mathrm{~d}$ slice only. Two-dimensional simulations are much less computer-intensive than three-dimensional simulations and are therefore preferred as long as three-dimensional effects can be neglected.

In some situations though, three-dimensional effects need to be taken into account. The approximation of an infinitely long beam becomes invalid for short beams, and a threedimensional simulation will be necessary for accurate results. Bunch compression can still be approximately simulated in two dimensions by increasing the beam current as a function of longitudinal distance $z$. Three-dimensional simulations will be needed though to validate the results from the two-dimensional simulations.

\subsubsection{Presence of Background Gas and Electrons}

As the beam enters the target chamber, beam ions will collide with molecules in the background gas, mainly consisting of $\mathrm{BeF}_{2}$, the most volatile constituent of the molten salt Flibe [75]. In addition to scattering, background ionization and beam stripping may occur, which can raise the charge state of the ion appreciably. According to recent results [2], for the specific case of a $20 \mathrm{MeV}$ per nucleon lead beam, initially in a +1 charge state,

entering a typical HIF chamber containing a background gas of $5 \times 10^{13} \mathrm{~cm}^{-3} \mathrm{BeF}_{2}$, the 
charge state is predicted to increase to about +10 after 3 meters, and between +13 and +14 after 5 meters. To assess the effect of stripping on the beam dynamics though, we need to consider the behavior of the stripped electrons in the target chamber. 


\section{Chapter 3}

\section{Simulation Codes}

Detailed analysis of space-charge dominated beams is unimaginable without relying on sophisticated computer codes to simulate the dynamics of the beam. Even the envelope equations $(2.44),(2.45)$ form a pair of coupled nonlinear differential equations that in general can only be solved numerically. In order to consider the high-intensity beams needed for heavy-ion inertial fusion, we need to take nonlinear space charge and other nonideal effects into consideration. A simple envelope solution will not suffice. Instead, the dynamics of the beam can be studied by calculating the trajectories of many macroparticles, each representing a large number of actual beam particles. Particle-in-cell codes such as WARP, described below, follow this principle. Alternatively, the beam dynamics can be studied by calculating the evolution of the distribution function in phase space based on the Vlasov equation. This approach generally yields higher accuracy in low-density regions of phase space. However, the computational power required by these Vlasov methods makes them unattractive for the beam dynamics problems we will be concerned with here. 
In this chapter, the simulation codes are described that were used for the beam physics problems treated in this thesis. The envelope solver JavaEnvelope, the suite of particle-in-cell codes WARP, as well as the fluid codes CIRCE and Hermes are discussed.

\subsection{JavaEnvelope}

The JavaEnvelope code was originally written by William Fawley and Jason Novotny of Lawrence Berkeley National Laboratory and was further developed by the author. This code integrates the envelope equations (2.44), (2.45) for a focusing lattice and initial beam parameters specified in an inputfile, and draws the calculated horizontal and vertical beam radius as a function of the longitudinal distance $z$ onto the screen. The lattice may consist of drift spaces and quadrupoles, as well as neutralization points where the charge of the beam is changed by some specified fraction. The graphical user interface, which allows the user to change the initial beam parameters and the focusing strengths of the quadrupoles, and the rapid envelope calculation and visual feedback make JavaEnvelope a convenient tool for initial lattice designs. In addition, the code is equipped with a matching routine to calculate the focusing strengths of four quadrupoles that will match a beam to a given set of final beam parameters. The JavaEnvelope code is written in Java 2, and runs under Unix, Linux, and Windows. Earlier versions written in Java 1.1.6 run on Macintosh computers. 


\subsection{WARP}

WARP is a suite of simulation codes that represent a hierarchy of levels of analysis. It was written to analyze beam physics problems for heavy ion inertial fusion, in particular taking into account the nonlinear self-fields of space-charge dominated beams [34], [42]. The name "WARP" arises from its ability to handle bends in accelerator systems, which are needed in heavy-ion inertial fusion applications [33].

WARP is used as a module for the Python interpreter language [95], which makes it highly flexible as it allows user-programmed code steering. The WARP code relies on the numerical package NumPy for Python [3], and makes use of the Python Gist package for graphics [76]. WARP's various components can be loaded and used as a code by itself by calling the appropriate routines from the Python interpreter. As an example, an initial calculation using WARP's envelope solver to set up a focusing lattice may be followed by a more detailed particle-in-cell calculation. In addition, side calculations can be carried out in Python using the numerical routines available in NumPy when needed.

WARP uses a Python script as its inputfile. It typically contains all the information about the focusing lattice and the initial beam parameters, as well as the commands that run the actual simulation. Upon finishing a script, WARP can generate a dumpfile containing all the information needed to continue the simulation at a later time. Furthermore, postprocessing as well as additional calculations can be performed by calling the appropriate WARP, Gist, NumPy, or native Python routines from the Python interpreter.

WARP runs on Cray computers, Sun workstations and Linux machines. It has been parallelized for the Cray T3E-900 and the IBM RS/6000 SP supercomputers. 


\subsubsection{Envelope Solver}

WARP contains an envelope solver, which calculates the horizontal and vertical envelope of a beam going through a focusing lattice. The envelope solver can be used to check the lattice specification in the input file before running the full particle simulation, as well as for initial matching. The envelope solver uses the isochronous leap-frog algorithm described below to integrate the envelope equations. The undepressed and depressed phase advance defined in equations (2.51) and (2.57) are also calculated. The envelope solver is able to handle off-axis beams, as well as a changing current and emittance whose $z$ dependence is specified by the user. Several Python scripts exist that make use of WARP's envelope solver to match a beam to a given transport lattice, or to find the focusing strengths needed for four quadrupoles to match a beam to a given set of final beam parameters.

\subsubsection{WARP3d, WARPxy, and WARPrz}

Originally, WARP was written as a fully three-dimensional particle-in-cell code to analyze the behavior of high-intensity beams for heavy-ion inertial fusion. The threedimensional code is now known as WARP3d; a two-dimensional package WARPxy was written to model one transverse slice of a beam going through a focusing system. As long as longitudinal effects are negligible, using WARPxy is preferable to WARP3d for its speed. Note that some longitudinal effects can be modeled to some degree even in the transverse slice code. For instance, longitudinal compression of a beam can be partially modeled in WARPxy by increasing the weight of each simulation particle as the line charge density increases. Many WARPxy runs can then be validated with a few WARP3d simulations. 
In many situations, the system to be studied exhibits some form of symmetry in the transverse plane. The run time needed in WARP can be reduced substantially by using two-fold or four-fold symmetry for the calculation of the space-charge field. This means that the potential is calculated in only one half or one quadrant of the system, into which the positions of the particles located in the other half or quadrants are mapped. The spacecharge force exerted on the particles is then determined based on their mapped positions in the half or quadrant where the potential was calculated.

WARP calculates the particle trajectories using the leap-frog algorithm, in which the positions and velocities of the particles are advanced alternately. Figure 3.1 shows a schematic of the leap-frog algorithm [10]. Notice that the positions and velocities of the particles are known at different points in time. However, at those time steps where the user may access the particles and where diagnostics are employed, WARP applies an isochronous advance. This means that the velocity advance is split into two steps, such that after one half-step the position and velocity are known at the same point in time [40]. The size of each leap-frog step is specified by the user, and can vary for different parts of the lattice depending for instance on how fast the focusing fields vary axially. For the WARP simulations presented in this thesis, typically about fifty steps were used per half period.

On every time step, the space-charge forces acting on the beam particles need to be calculated. The self-field is calculated in the beam frame using the particle-in-cell method, in which the charge of the beam particles is deposited onto a rectangular grid, followed by solving Poisson's equation using the Fast Fourier Transform method. Since the self-field is assumed to be electrostatic in the beam frame, only Poisson's equation needs to be solved. 


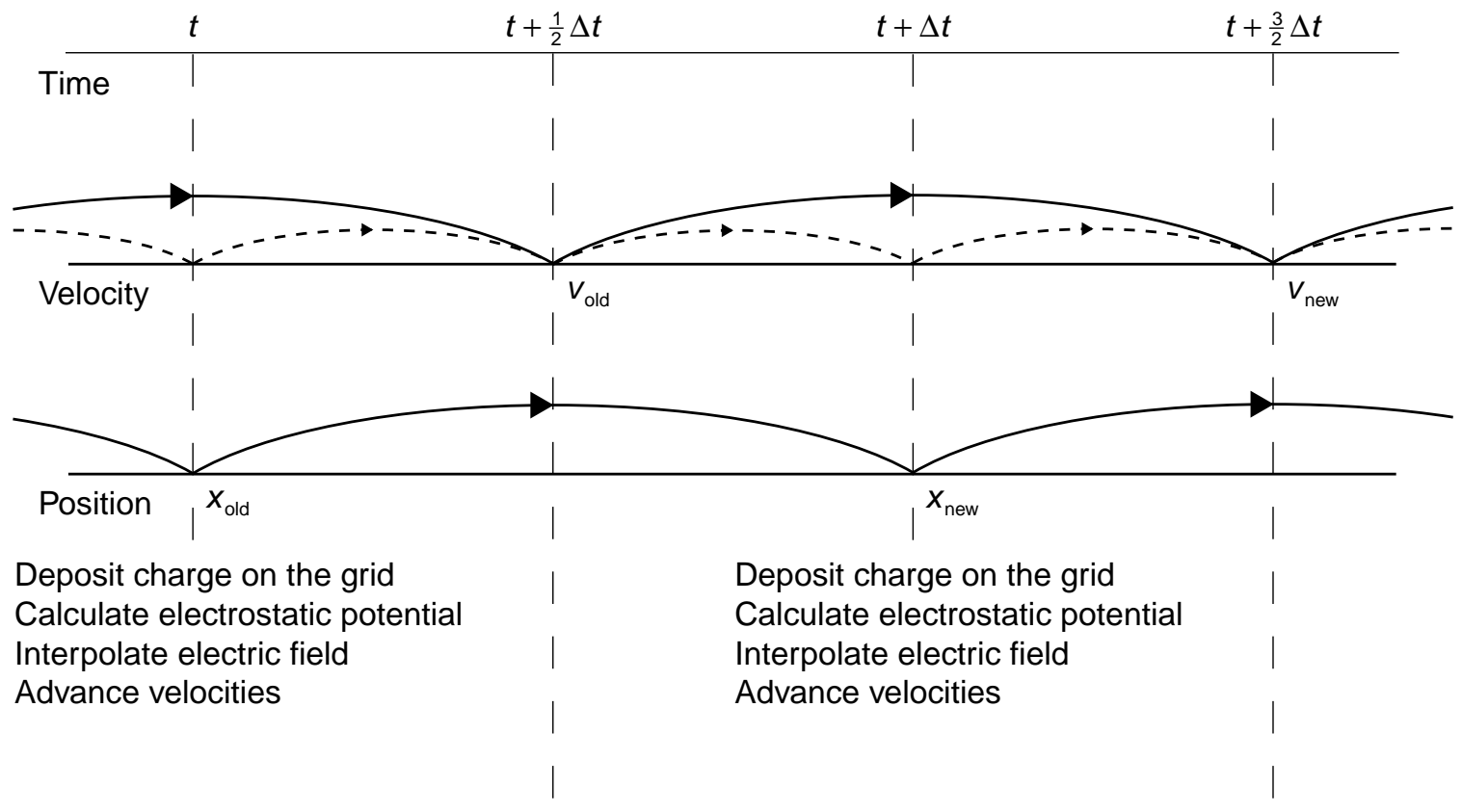

Advance positions

Advance positions

Figure 3.1: Schematic of the leap-frog algorithm [10]. The dashed line represents the isochronous leap-frog algorithm.

The grid size is specified by the user; for the WARPxy simulations presented here, typically a grid size of $128 \times 128$ was used. A finer grid improves the accuracy of the space-charge force, but also increases the required computing time.

Longitudinally, WARP3d uses the usual Fourier transform to solve Poisson's equation. This implicitly assumes that the potential is periodic. This assumption is reasonable as long as the beam does not approach the ends of the grid [33], because the surrounding conducting walls effectively shield the virtual beams produced by the transform.

Transversely, both WARP3d and WARPxy use a sine-transform instead of the usual Fourier transform to solve Poisson's equation. This effectively puts an artificial rectangular conducting wall around the edges of the grid. Image charges on such a rectangular conducting wall could significantly distort the beam. This artificial wall should therefore 
be carefully shielded in the simulation. This can be done either by representing in the simulation the actual conducting boundaries, or by adding a fictitious circular conducting boundary that just fits inside the simulation grid. Such conducting boundaries can be modeled in WARP by the capacity matrix technique [41], [42], [49]. By accurately representing the presence of conducting boundaries using the capacity matrix technique, the effect of image charges on the beam are included properly in the simulation. This may be particularly of importance for off-axis beams.

In the presence of complex conducting boundaries, the capacity matrix may become too large to be solved in a reasonable amount of time. For those cases, a successive overrelaxation (SOR) and a multi-grid Poisson solver are available in WARP3d [42]. For the simulations presented here, these solvers were not needed. Any non-conducting materials that may be present near the beam, such as the Flibe jets in the target chamber, were not included in the simulations. Their behavior may be quite complex due to their dielectric properties, as well as due to the possibility of flash ionization.

The lattice elements may consist of accelerating gaps, quadrupoles, as well as generally defined multipole elements. Accelerating gaps can have a user-defined time-dependent accelerating field. The generic quadrupole elements are hard-edged, and are most convenient to use if higher-order multipoles are absent and any axial dependence of the field of each quadrupole is ignored. For hard-edged elements with an axially uniform field, the leapfrog algorithm as implemented in WARP employs residence corrections. This means that the impulse received by the beam particles going through a focusing element is corrected for the time actually spent by each particle inside the element. The multipole elements 
are $z$-dependent and are more generally defined in terms of the parameters $n$ and $\nu$ as in equations (2.9)-(2.14). The multipole fields can be given a rotation angle, which can be used to include the effect of the skew quadrupole component in a slightly rotated quadrupolar magnet. The quadrupoles as well as the multipole fields can be placed off-axis in the simulation in order to represent misaligned focusing elements.

A Python script is available to set up the required multipole fields as a function of $z$ for a quadrupole with a given longitudinal fall-off in the focusing fields near its ends. In this script, by default the pseudo-octupole $e / b_{2,1}(z)$ and its accompanying axial field $e / b_{2,0}^{\prime}(z)$ are set up in addition to the $z$-dependent quadrupolar field $e / b_{2,0}(z)$. Additional higher-order multipoles can be included if desired. The script assumes by default that the fringe fields have the fall-off given by equation (2.73), with a fringe length given by the user. Alternatively, the script can handle a user-defined fall-off shape.

The initial number of macroparticles is specified by the user in the inputfile. For the WARPxy simulations described in this thesis, typically about 40,000 macroparticles were used. If a particle strays off the center of the beamline farther than an aperture distance specified by the user, it is removed from the simulation. Although some particles will be lost in a simulation due to halo formation, the loss of too large ( $\gtrsim 1 \%)$ a fraction of the particles may indicate that the simulation was insufficiently accurate.

The accuracy of a particle-in-cell simulation depends on the number of macroparticles, the number of grid cells, and the step size that was used in the numerical integration. Typically for a WARPxy simulation, about 100 macroparticles per grid cell with 50 steps per lattice half period are used, whereas the grid size is chosen such that about 10 grid cells 
cover the beam transversely. The convergence of a particle-in-cell simulation can be verified by comparing its results with those of a run using a larger number of macroparticles, a finer grid, or a smaller step size.

The initial distribution of these particles can be either K-V (equation (2.24)) or semi-Gaussian (equation (2.50)), as well as an arbitrary particle distribution dependent on the radial coordinate $r$. It is also possible to modify the particle arrays in WARP from the Python interpreter directly in order to model any arbitrary particle distribution. For the initial particle load in the simulations described here, the digit-reversed method was used as a non-random number generator. The initial beam distribution can be placed off-axis in order to simulate misaligned beams.

In addition to WARP3d and WARPxy, WARP contains a package WARPrz for simulations of cylindrically symmetric systems. Although no WARPrz simulations were done for this thesis work, the fluid/envelope code Hermes discussed in section 3.2.4 makes use of the field solver in WARPrz to find the longitudinal electric field in the Hermes model.

\subsubsection{The CIRCE Module}

CIRCE is a fast-running 3D envelope code [89], which was originally written as a stand-alone code but is now incorporated into WARP. CIRCE was originally written for the Small Recirculator Experiment at Livermore [31]; its acronym stands for "Calculation of Ion Recirculation by Compressible Envelopes". In the CIRCE model, the beam is divided into many axial slices, each having a fixed charge and a constant transverse normalized emittance. The slices are separated from each other by slice boundaries. The transverse beam dynamics is then found by integrating the envelope equations $(2.44),(2.45)$ for each 
slice boundary separately, including both the perveance and the emittance terms. The lowest-order effect of image fields from a circular beam pipe of infinite conductivity can also be included in the CIRCE calculation. Higher-order multipole fields are neglected though, as appropriate for an envelope solver.

For the longitudinal dynamics, the axial beam slices are treated as Lagrangian fluid elements. The slice boundaries, identified by an index $i$, are characterized by an axial velocity $v_{i}$, and an arrival time $t_{i}$ for a given $z$ location. Typically about one hundred slices are used. Each slice $i+\frac{1}{2}$ contains a fixed charge $\Delta Q_{i+\frac{1}{2}}$. This approach implicitly assumes that the longitudinal temperature of the beam is negligible [89]. The CIRCE model used for the longitudinal dynamics is shown in figure 3.2 .

The longitudinal equation of motion can be written as

$$
m \gamma^{3} \frac{\mathrm{d} v}{\mathrm{~d} t}=q e E
$$

in which $m \gamma^{3}$ is the longitudinal mass [83] and $E$ is the longitudinal electric field. It consists of the externally applied longitudinal field $E_{\text {ext }}$ and the longitudinal space-charge field $E_{\mathrm{sp}}$ due to the beam itself.

Several models are available in CIRCE to find the longitudinal force $E_{\mathrm{sp}}$ generated by the space charge of the beam. These models are based on the $g$-factor model, which can be derived as follows. The potential of an infinite beam of circular cross section with radius $a$, surrounded by a conducting circular pipe of radius $R$, is given by

$$
\Phi^{\prime}= \begin{cases}-\frac{\lambda^{\prime}}{2 \pi \epsilon_{0}} \ln \left(\frac{r}{R}\right) & \text { outside the beam } \\ -\frac{\lambda^{\prime}}{4 \pi \epsilon_{0}}\left(\frac{r^{2}}{a^{2}}-1\right)-\frac{\lambda^{\prime}}{2 \pi \epsilon_{0}} \ln \left(\frac{a}{R}\right) & \text { inside the beam }\end{cases}
$$

in which primed variables are taken in the beam frame. Note that $r, R$, and $a$ are transverse 


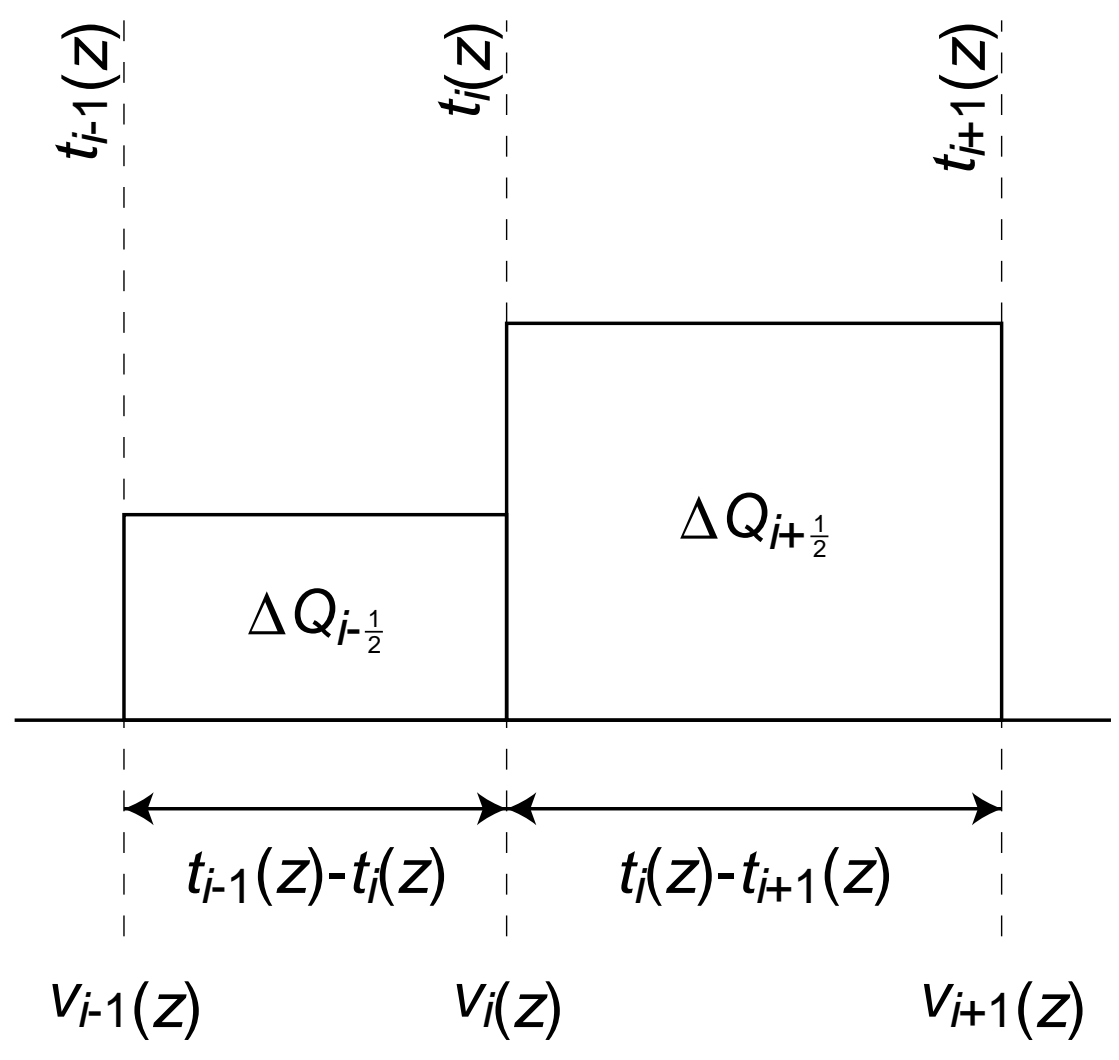

Figure 3.2: Schematic of the CIRCE model.

dimensions, which are the equal in the beam frame and in the laboratory frame. By taking the partial derivative of $\Phi$ inside the beam with respect to $z^{\prime}$, we find the longitudinal electric field there:

$$
E_{\mathrm{sp}}^{\prime}=\frac{1}{4 \pi \epsilon_{0}}\left(\frac{r^{2}}{a^{2}}-1\right) \frac{\partial \lambda^{\prime}}{\partial z^{\prime}}+\frac{1}{2 \pi \epsilon_{0}} \ln \left(\frac{a}{R}\right) \frac{\partial \lambda^{\prime}}{\partial z^{\prime}}-\frac{\lambda^{\prime}}{2 \pi \epsilon_{0}} \frac{r^{2}}{a^{3}} \frac{\partial a}{\partial z^{\prime}}+\frac{\lambda^{\prime}}{2 \pi \epsilon_{0} a} \frac{\partial a}{\partial z^{\prime}} .
$$

In space-charge dominated beams, the electric field dominates over the thermal motion of the beam ions. Nonuniformities in the space-charge density will then fade out rapidly due to the electric field that they generate. We will therefore assume that space-charge dominated beams have a uniform space-charge density $\rho$, which means that $\lambda^{\prime} / a^{2}$ is constant along the 
beam. We can then express $\partial a / \partial z^{\prime}$ in terms of $\partial \lambda^{\prime} / \partial z^{\prime}$ :

$$
\frac{1}{a} \frac{\partial a}{\partial z^{\prime}}=\frac{1}{2} \frac{1}{\lambda^{\prime}} \frac{\partial \lambda^{\prime}}{\partial z^{\prime}}
$$

Substitution then yields

$$
E_{\mathrm{sp}}^{\prime}=\frac{1}{2 \pi \epsilon_{0}} \ln \left(\frac{a}{R}\right) \frac{\partial \lambda^{\prime}}{\partial z^{\prime}}
$$

In the laboratory frame, we can write this equation as

$$
E_{\mathrm{sp}}=\frac{1}{2 \pi \epsilon_{0} \gamma^{2}} \ln \left(\frac{a}{R}\right) \frac{\partial \lambda}{\partial z}
$$

taking into account the Lorentz contraction in the longitudinal dimension. We can write this in the form

$$
E_{\mathrm{sp}}=-\frac{g}{4 \pi \epsilon_{0} \gamma^{2}} \frac{\partial \lambda}{\partial z}
$$

if we define the $g$-factor as

$$
g \equiv 2 \ln \left(\frac{R}{a}\right)
$$

For elliptical beams with axes $a$ and $b$, the generalization of this equation is

$$
g=\ln \left(\frac{R^{2}}{a b}\right)
$$

Near the ends of the beam, the $g$-factor model begins to break down because of the rapid variation in beam radius along the beam. More accurate models, which include the variation of the beam radius, are used there. Notice that the longitudinal and transverse dynamics are coupled through the perveance (which depends on the longitudinal expansion or compression of the beam) and the $g$-factor. 


\subsubsection{The Hermes Project}

Although the CIRCE module described in the previous section is useful for rough studies of longitudinal beam dynamics, if a large $(\gtrsim 50$ for the IRE drift compression calculations described in chapter 4) number of slices is used, the code tends to produce unphysical results due to slices overtaking each other. It is therefore difficult to perform higher-precision calculations with a larger number of slices to check the accuracy of the results. Slice overtaking, often followed by code crashes, usually takes place near the ends of the beam, although CIRCE may be calculating the longitudinal dynamics in other parts of the beam correctly.

There are two possible causes for slice overtaking to occur. First, CIRCE is based on a fluid model, which may be invalid in the physical regime we are interested in. Particularly, longitudinal acoustic waves occurring in the beam may steepen into shock waves due to the nonlinear nature of the fluid equations. The fluid model may then break down as fluid properties such as the velocity become double-valued [90]. Although shocks may be modeled in a fluid code by using artificial viscosity [84], [90], the results from such a code do not necessarily agree with a kinetic calculation.

Secondly, the longitudinal electric field in CIRCE may not be calculated accurately, particularly near the beam ends, where the $g$-factor model breaks down. Although more advanced field solvers are available in CIRCE, they rely on knowing the distance from a slice boundary to the ends of the beam. This can only be calculated in approximation from the arrival times by extrapolating the slice boundary positions forward and backward in time. In addition, these models still break down near the beam tips, where slice overtaking 
usually takes place.

In order to deal with these issues, a time-dependent code Hermes was written based on CIRCE. In the Hermes model, the positions of the different slice boundaries are calculated at a given time, instead of the arrival times at a given position, which is done in CIRCE. This is more suitable for a reliable calculation of the longitudinal electric field for three reasons. First, the $g$-factor model can be implemented more easily in such a code, since it depends on derivatives with respect to $z$. Also the more advanced models of CIRCE can be implemented in Hermes without the need to extrapolate the position of the beam tips from the arrival times. The biggest advantage over CIRCE is though that since we have the position of all the slice boundaries available at each time step, we can find the longitudinal electric field directly from the charge density by solving Poisson's equation.

The Hermes model is shown in figure 3.3. Each slice boundary still follows its own transverse envelope equation. For the calculation of the longitudinal electric field, Hermes assumes the beam is cylindrically symmetric. It then acts as an RZ code by using the WARPrz package to calculate the longitudinal electric field. This allows us to find the longitudinal electric field accurately even near the beam ends. This approach eliminates the need to rely on the $g$-factor model, which breaks down near the ends of the beam.

To calculate the longitudinal electric field, the charge of each slice is deposited onto an RZ grid. We assume that each slice has a uniform charge density and is cylindrical with a radius equal to $\sqrt{a b}$, where $a$ and $b$ are the horizontal and vertical beam radius of the slice. An infinitesimal ring of charge $\mathrm{d} q=2 \pi r \mathrm{~d} r \mathrm{~d} z$ between longitudinal positions $z$ and $z+\mathrm{d} z$ and radii $r$ and $r+\mathrm{d} r$ is then deposited onto the grid using a volume weighting 
scheme, shown in figure 3.4. We then integrate the infinitesimal rings of charges over each slice, and sum over the slices, to determine the charge density to be associated with each grid point. For a grid point $i, j$ at position $r_{i}, z_{j}$ and a slice with charge density $\rho$, this yields

$$
\begin{aligned}
\rho_{0, j} & =2 f_{j} \rho \\
\rho_{i, j} & =f_{j} \rho \text { for } i<n \\
\rho_{n, j} & =f_{j} \frac{\rho}{n}\left(\frac{1}{4}(2 n-1)-\frac{\xi}{2 n+1}\left(n+\frac{1}{2} \xi\right)\left(\frac{1}{2} \xi^{2}+n \xi-1-2 n\right)\right), \\
\rho_{n+1, j} & =f_{j} \frac{\rho}{(n+1)(2 n+1)} \xi^{2}\left(n+\frac{1}{2} \xi\right)^{2}, \\
\rho_{i, j} & =0 \text { for } i>n+1
\end{aligned}
$$

in which $n=$ floor $\left(\frac{\sqrt{a b}}{\Delta r}\right), \xi=\frac{\sqrt{a b}}{\Delta r}-n$, and $f_{j}$ is a scaling factor which is equal to unity if the slice covers both axially neighboring grid cells, and less than unity otherwise, as shown in figure 3.5. This weighting scheme conserves the RMS beam radius. Note that a grid point on axis is assigned twice the density.

To find the potential, we use the Poisson solver for cylindrically symmetric systems from Warp's RZ package [19]. This field solver uses the periodic FFT transform longitudinally, and a tridiagonal difference scheme radially. Longitudinally, the grid size is equal to the length of the beam plus four times the pipe radius on either side in order to shield fields from the virtual beams that exist due to the periodic nature of the FFT. The radial grid size is equal to the largest aperture present on this domain. The longitudinal electric field acting on a slice boundary is then calculated from

$$
E_{z}=\frac{\Delta z_{+}-\frac{1}{2} \Delta z}{(\Delta z)^{2}} \Phi_{-}+\frac{\Delta z_{-}-\Delta z_{+}}{(\Delta z)^{2}} \Phi_{0}+\frac{-\Delta z_{-}+\frac{1}{2} \Delta z}{(\Delta z)^{2}} \Phi_{+}+O\left(\Delta z^{2}\right)
$$




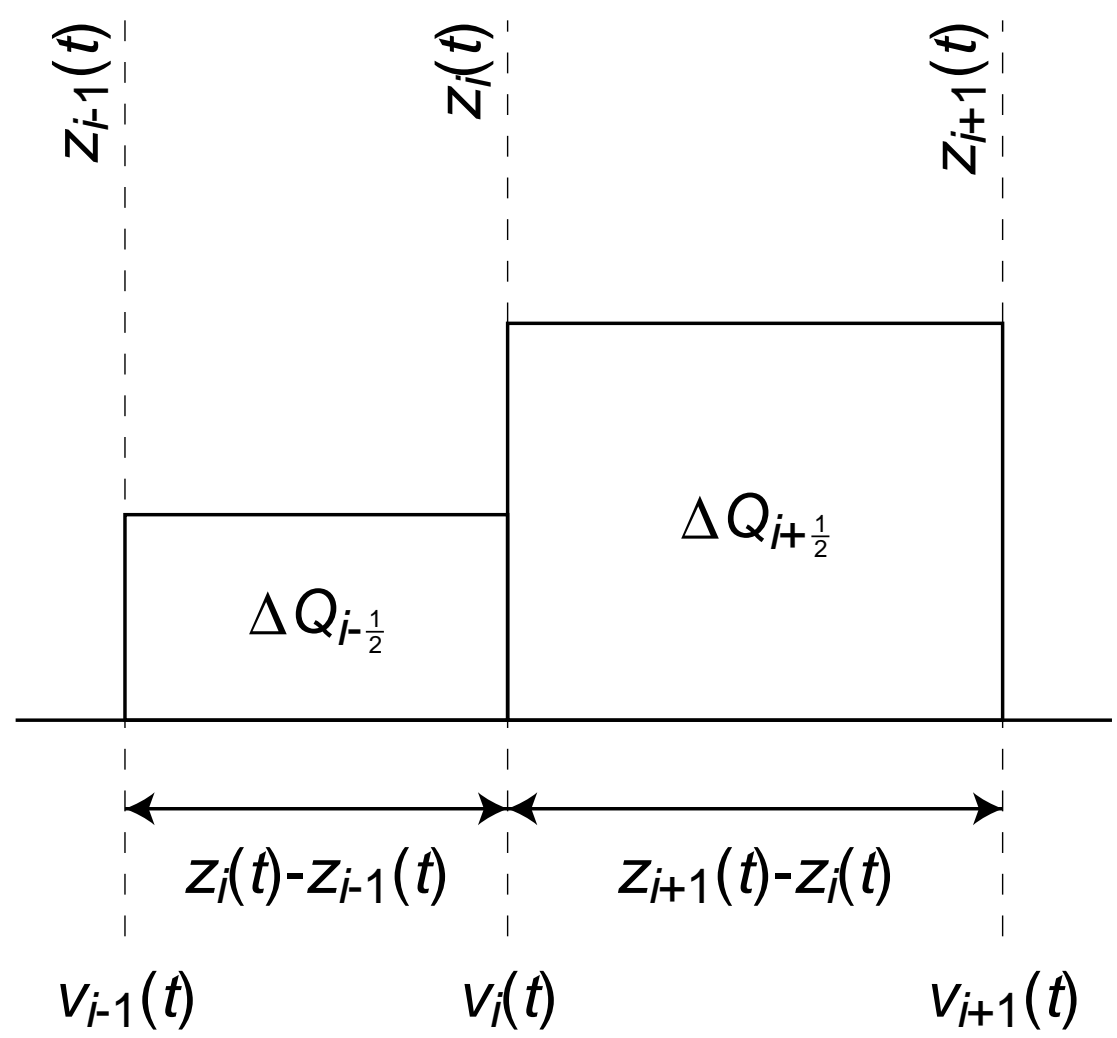

Figure 3.3: Schematic of the Hermes model.

(see figure 3.6). The Hermes code can calculate the longitudinal electric field either on axis, or as an average over the cross section of the beam, using the same weighting scheme as for the charge deposition.

Hermes has the option of using artificial viscosity to be able to handle shocks. Artificial viscosity in Hermes is based on Richtmyer's approach [84], in which a viscous force of the form

$$
F_{\text {viscous }}= \begin{cases}-m \ell^{2} \frac{\rho_{0}}{\rho} \frac{\partial}{\partial z}\left(\frac{\rho_{0}}{\rho}\left(\frac{\partial v}{\partial z}\right)^{2}\right) & \text { if } v \frac{\partial v}{\partial z}<0 \\ 0 & \text { if } v \frac{\partial v}{\partial z}>0\end{cases}
$$

is added to the longitudinal equation of motion. In this equation, $\rho$ is the local charge 


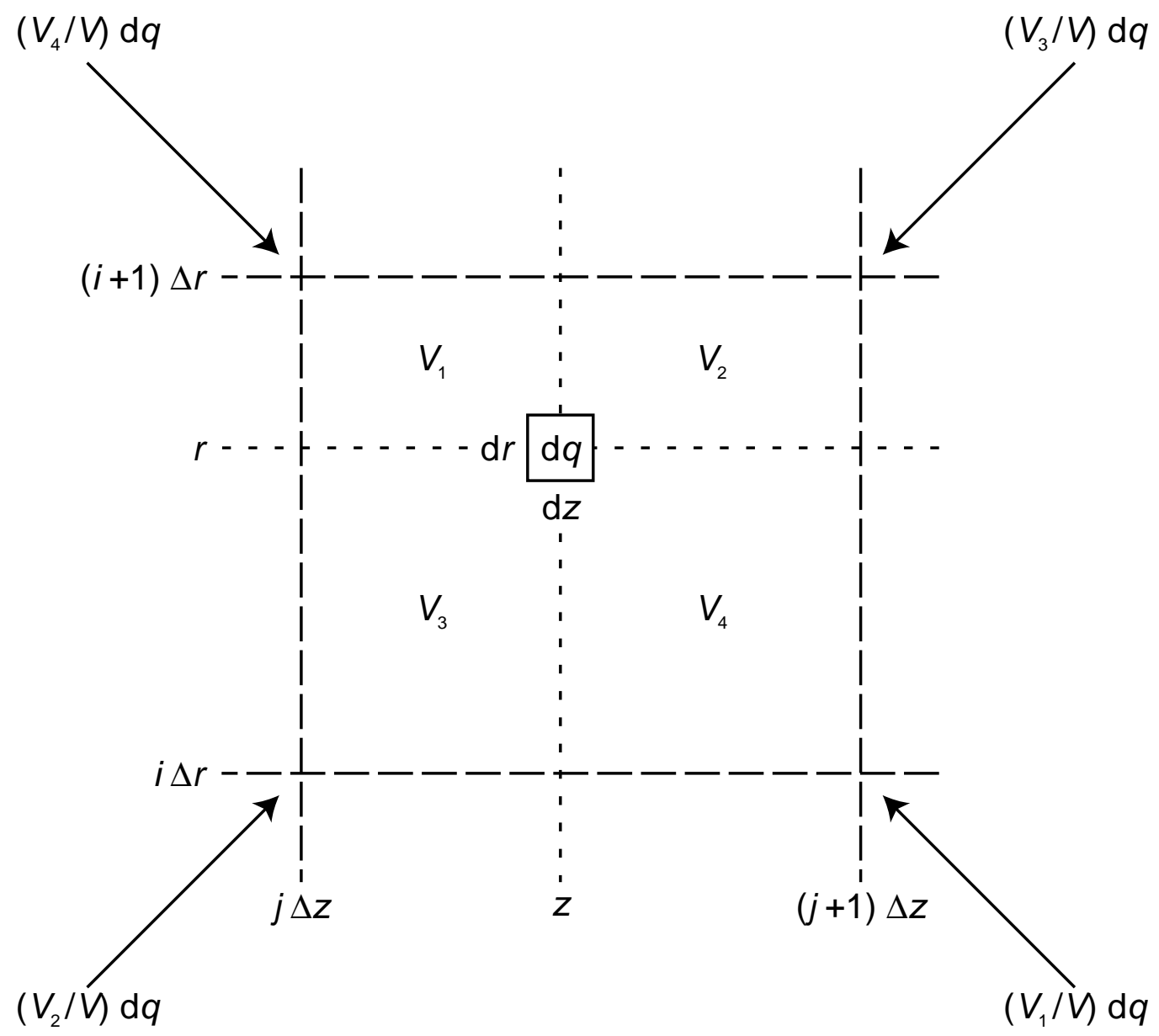

Figure 3.4: The volume weighting scheme used in Hermes for charge deposition. The volumes are calculated as

$$
\begin{aligned}
V_{1} & =[z-j \Delta z] \pi\left[((i+1) \Delta r)^{2}-r^{2}\right], \\
V_{2} & =[(j+1) \Delta z-z] \pi\left[((i+1) \Delta r)^{2}-r^{2}\right], \\
V_{3} & =[z-j \Delta z] \pi\left[r^{2}-(i \Delta r)^{2}\right] \\
V_{4} & =[(j+1) \Delta z-z] \pi\left[r^{2}-(i \Delta r)^{2}\right] \\
V & =V_{1}+V_{2}+V_{3}+V_{4} \\
& =\pi\left[(i+1)^{2}-i^{2}\right](\Delta r)^{2} \Delta z .
\end{aligned}
$$



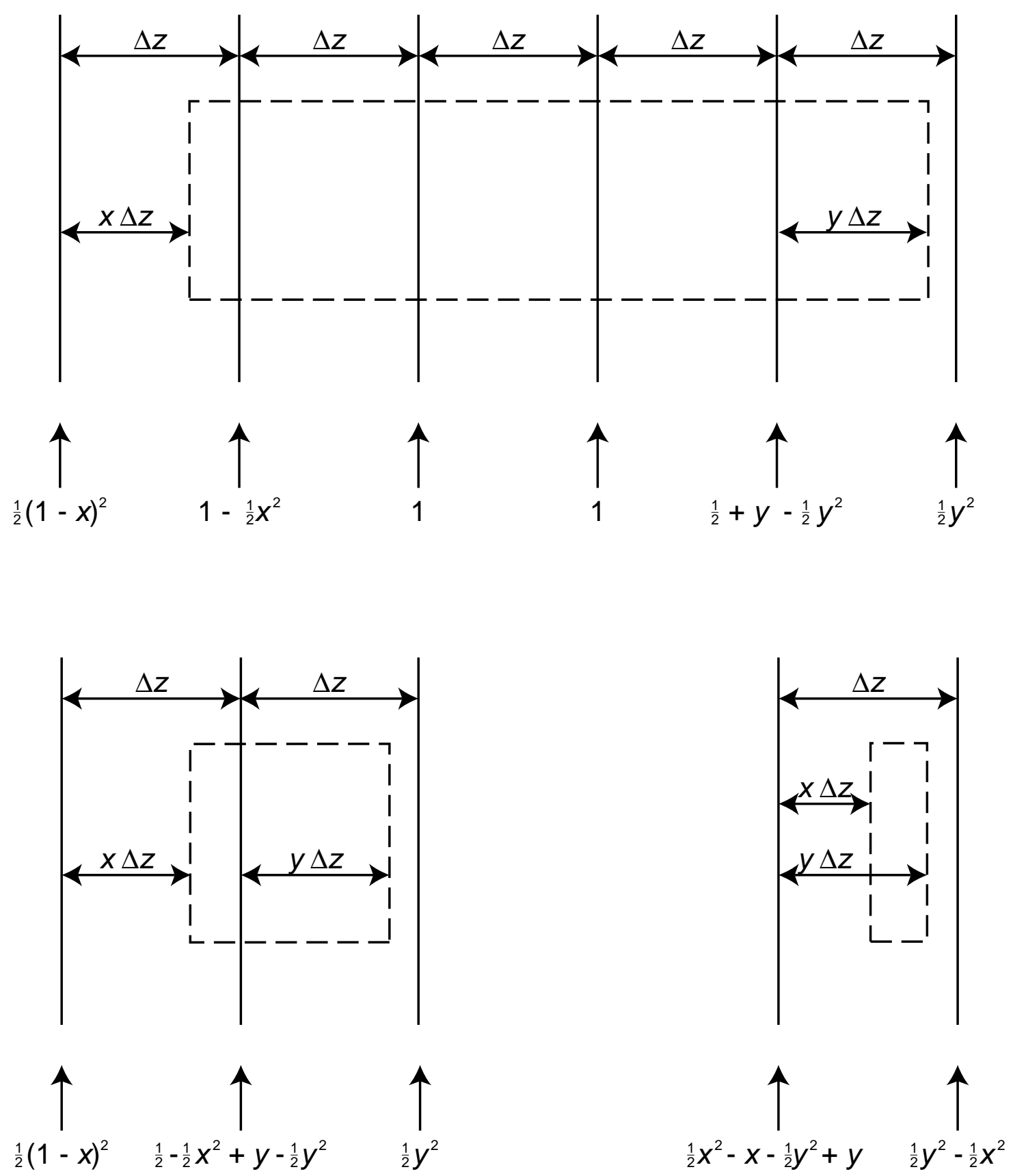

Figure 3.5: Scaling factor $f_{j}$ for the charge deposition described by equations (3.10) through (3.14), as determined by the relative position of the slice boundaries to the grid points. 


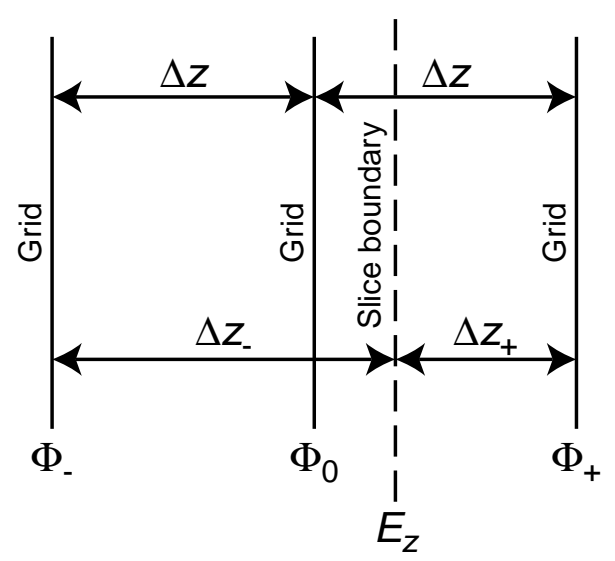

Figure 3.6: Interpolation scheme for the longitudinal electric field in Hermes (equation $(3.15))$.

density, whereas $\rho_{0}$ is some global measure of the charge density. In Hermes, $\rho_{0}$ was set equal to the charge density at the center of the beam. The quantity $\ell$ is the typical distance over which a shock is smeared out in the calculation, and should be set equal to a couple of slice widths. For the calculations presented in this thesis, artificial viscosity was not used since no shocks occurred.

\section{Test Cases}

The $g$-factor model as described in section 3.2.3 is valid for beams that are long compared to their radius. To check the validity of the Hermes model, a long test beam was simulated in a periodic focusing lattice, and the simulation results were compared to the $g$-factor model. The beam parameters are listed in table 3.1, whereas the lattice parameters are given in table 3.2. These parameters correspond to an IRE beam just before the final focus system, if we assume a pulse duration of $100 \mathrm{~ns}$ (chapter 4). The current profile was flat, with parabolic ends of $5 \%$ of the beam length on each side. The lattice was set up for a $72^{\circ}$ undepressed tune with $65 \%$ occupancy, which is what we expect just before final 


\begin{tabular}{|l|l|}
\hline Ion & ${ }^{39} \mathrm{~K}^{+}$ \\
\hline Beam energy & $200 \mathrm{MeV}$ \\
\hline Edge emittance & $9.52 \pi \mathrm{mm} \mathrm{mrad}$ \\
\hline Initial beam current & $46.875 \mathrm{~A}$ \\
\hline Initial beam length & $3.13 \mathrm{~m}$ \\
\hline Initial beam duration & $100 \mathrm{~ns}$ \\
\hline Initial beam radius & $59.1 \mathrm{~mm}$ \\
\hline Initial perveance & $6.67 \cdot 10^{-5}$ \\
\hline
\end{tabular}

Table 3.1: Beam parameters of the 100 ns test beam.

\begin{tabular}{|l|l|}
\hline Lattice half period & $4.32 \mathrm{~m}$ \\
\hline Occupancy & $65 \%$ \\
\hline Focusing strength & $1.645 \mathrm{~T} / \mathrm{m}$ \\
\hline Undepressed tune & $72^{\circ}$ \\
\hline Pipe radius & $10.01 \mathrm{~mm}$ \\
\hline
\end{tabular}

Table 3.2: The transport lattice used for the 100 ns test beam.

focus. Unlike the drift compression schemes presented in chapter 4, the lattice properties were not adjusted for the change in current as the beam expands.

The simulation ran for twenty lattice half periods, using twenty steps per half period with one hundred slices. For the Hermes calculation, a $128 \times 128$ grid was used without spatial filtering. Figure 3.7 shows the current profile of the beam as calculated by the Hermes model and the $g$-factor model, after twenty half lattice periods. As expected the two models agree very well, except near the beam ends as well as in the transition region from the flat-top to the parabolic ends.

We expect the $g$-factor model to deviate from the Hermes model for short beams, since the $g$-factor model breaks down in that regime. We repeated the simulation described above for a test beam with a 5 ns initial duration. Its parameters are given in table 3.3. Note that the beam length of $156.6 \mathrm{~mm}$ is now of the same order of magnitude as the beam 


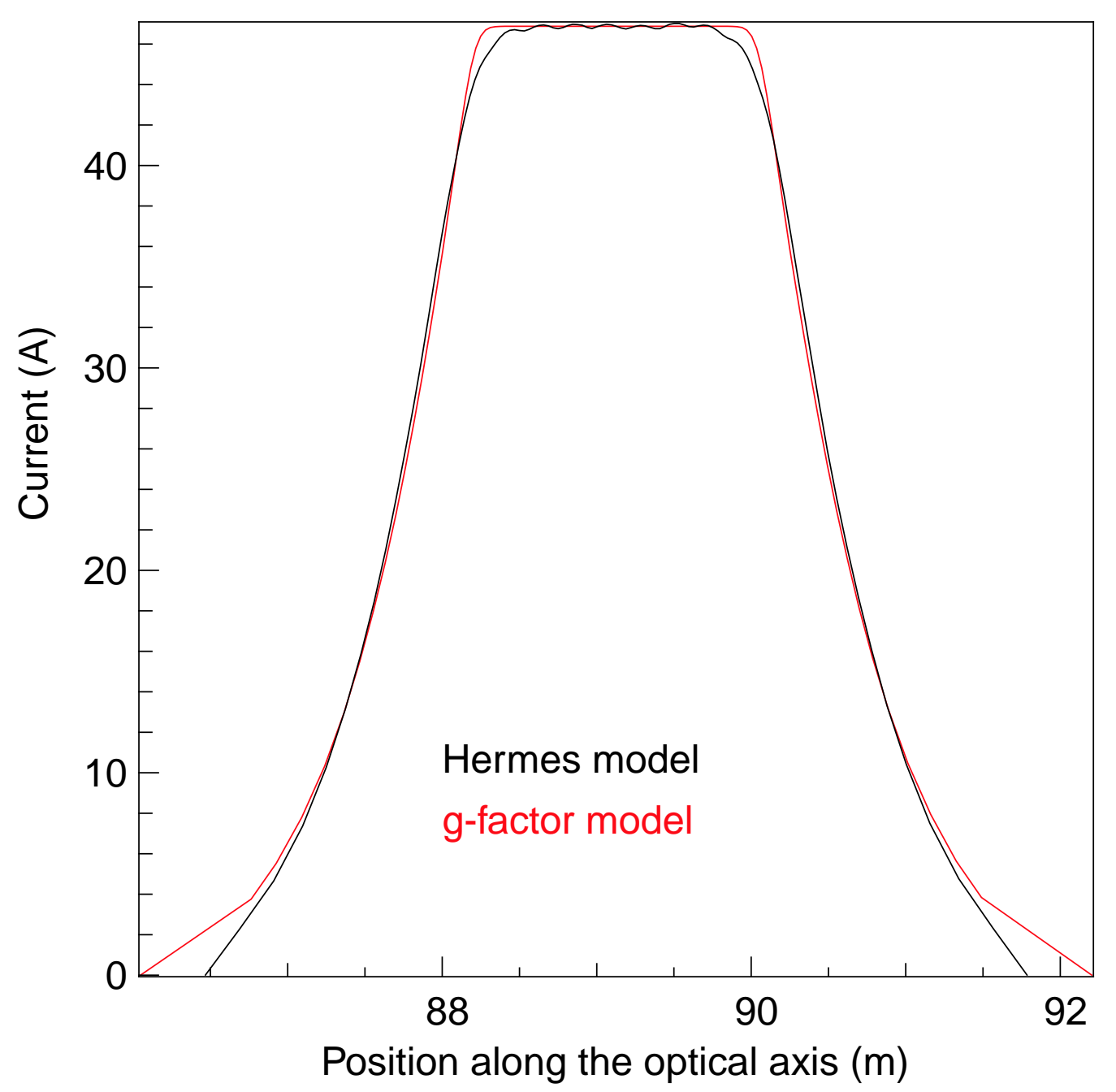

Figure 3.7: The beam current profile for an initially $100 \mathrm{~ns}$ beam of $46.875 \mathrm{~A}$, as calculated by the Hermes model and the $g$-factor model.

radius of $59.1 \mathrm{~mm}$. Again a flat current profile was used, with parabolic ends of $5 \%$ of the beam length each. The transport lattice for the 5 ns test beam is described in table 3.4.

The simulation ran for ten lattice half periods, using one hundred steps per lattice half period and a $1024 \times 256 \mathrm{RZ}$ grid for the Hermes model with one hundred slices. Due to the fast longitudinal expansion of the beam, the beam current decreases rapidly. In contrast to the drift compression calculations presented in chapter 4 , for this test case the lattice 
was not adjusted for the changing current, which then results in a decreasing average beam radius. After a couple of lattice half periods the beam radius becomes small compared to the pipe radius, which necessitates a very fine grid radially.

Figure 3.8 shows the beam current as a function of position along the lattice at a fixed time after traveling one, three, five and ten lattice half periods. The $g$-factor model significantly deviates from the Hermes model. This is due to the fact that for the $5 \mathrm{~ns}$ test beam, the assumption that the beam is long compared to its radius is no longer valid.

We can check the accuracy of the Hermes results by repeating the calculation using a larger number of slices, a larger number of steps, or a finer grid. Table 3.5 shows the current at the beam center after ten lattice half periods, using different numerical parameters. Since the difference in current between these cases is less than $0.12 \%$, we can conclude that the calculations described above were indeed converged. Figure 3.9 shows the current profile after four half lattice periods for a 5 ns beam using the numerical parameters indicated in table 3.5. This figure demonstrates that the difference in the calculated current profile is minimal for these cases.

\begin{tabular}{|l|l|}
\hline Ion & ${ }^{39} \mathrm{~K}^{+}$ \\
\hline Beam energy & $200 \mathrm{MeV}$ \\
\hline Edge emittance & $9.52 \pi \mathrm{mm} \mathrm{mrad}$ \\
\hline Initial beam current & $937.5 \mathrm{~A}$ \\
\hline Initial beam length & $156.6 \mathrm{~mm}$ \\
\hline Initial beam duration & $5 \mathrm{~ns}$ \\
\hline Initial beam radius & $59.1 \mathrm{~mm}$ \\
\hline Initial perveance & $1.33 \cdot 10^{-3}$ \\
\hline
\end{tabular}

Table 3.3: Beam parameters for the 5 ns test beam. 


\begin{tabular}{|l|l|}
\hline Lattice half period & $0.966 \mathrm{~m}$ \\
\hline Occupancy & $65 \%$ \\
\hline Focusing strength & $32.90 \mathrm{~T} / \mathrm{m}$ \\
\hline Undepressed tune & $72^{\circ}$ \\
\hline Pipe radius & $10.01 \mathrm{~mm}$ \\
\hline
\end{tabular}

Table 3.4: The transport lattice used for the 5 ns test beam.
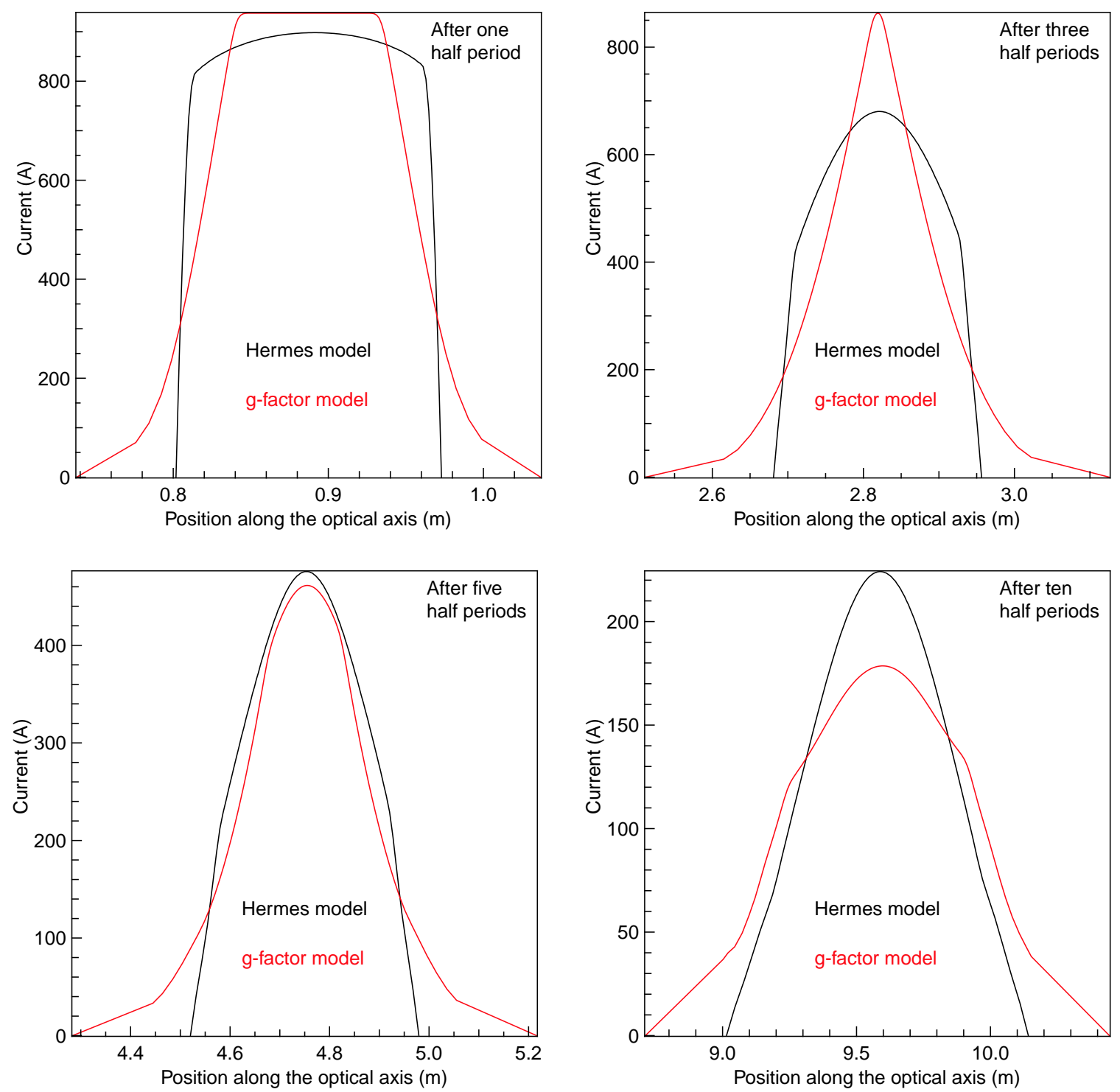

Figure 3.8: The beam current profile for an initially $5 \mathrm{~ns}$ beam of $937.5 \mathrm{~A}$ after traveling various distances along the transport lattice, as calculated by the Hermes model and by the $g$-factor model. 


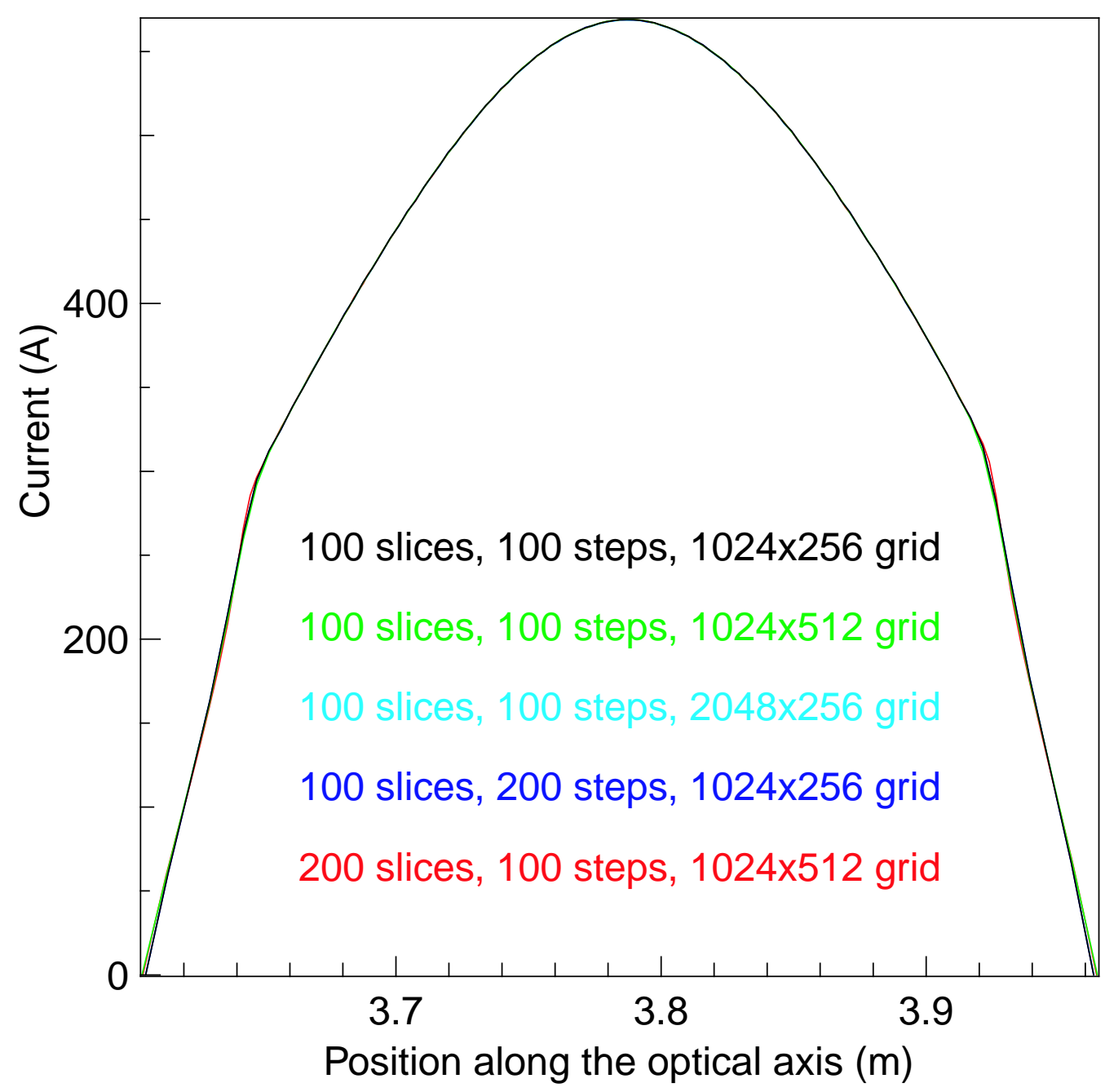

Figure 3.9: The beam current profile for an initially 5 ns beam of $937.5 \mathrm{~A}$ after traveling four lattice half periods, as calculated by the Hermes model. 


\begin{tabular}{|c|c|c|c|}
\hline $\begin{array}{c}\text { Number of } \\
\text { slices }\end{array}$ & $\begin{array}{c}\text { Number of steps } \\
\text { per half period }\end{array}$ & $\begin{array}{c}\text { RZ grid } \\
\text { size }\end{array}$ & $\begin{array}{c}\text { Current at } \\
\text { beam center }(\mathrm{A})\end{array}$ \\
\hline \hline 100 & 100 & $1024 \times 256$ & 224.15 \\
\hline 100 & 100 & $2048 \times 256$ & 223.90 \\
\hline 100 & 100 & $1024 \times 512$ & 224.15 \\
\hline 200 & 100 & $1024 \times 512$ & 224.18 \\
\hline 100 & 200 & $1024 \times 256$ & 224.13 \\
\hline
\end{tabular}

Table 3.5: The current at the beam center after traveling ten lattice half periods, for the 5 ns test beam, as calculated by Hermes using different numerical parameters.

\section{Comparison with WARP3d}

To assess the validity of the Hermes calculations, we can compare their results to 3D particle-in-cell simulations using WARP3d. There are three differences between the Hermes model and a WARP3d simulation. First, Hermes is based on a fluid model longitudinally, which means that one longitudinal velocity is associated with each fluid element. In contrast, WARP3d uses a kinetic model, in which different macroparticles in a given slice of the beam generally have different longitudinal velocities. As a consequence, in a kinetic model there exists an effective longitudinal pressure. Whereas Hermes takes the transverse pressure into account by means of the emittance term in the transverse envelope equation, no longitudinal pressure has been included so far in the Hermes model. Finally, for the calculation of the longitudinal electric field, the beam slices are approximated as being cylindrical in the Hermes model. This approximation is of course absent in WARP3d.

A comparison between Hermes and WARP3d was made for the 5 ns test beam described above. In order to minimize kinetic effects in the WARP3d simulation, the initial longitudinal emittance was set to zero. The beam was simulated for ten lattice half periods, using twenty steps per half period and 1,000,000 particles on a $128 \times 128 \times 512$ grid as the 


\begin{tabular}{|c|c|c|c|}
\hline $\begin{array}{c}\text { Number of particles } \\
\text { in millions }\end{array}$ & $\begin{array}{c}\text { Number of steps } \\
\text { per half period }\end{array}$ & $\begin{array}{c}\text { Grid } \\
\text { size }\end{array}$ & $\begin{array}{c}\text { Current at } \\
\text { beam center (A) }\end{array}$ \\
\hline \hline 1 & 20 & $128 \times 128 \times 512$ & 217.1 \\
\hline 1 & 20 & $256 \times 256 \times 512$ & 217.2 \\
\hline 1 & 40 & $128 \times 128 \times 512$ & 216.6 \\
\hline 1 & 20 & $128 \times 128 \times 1024$ & 218.6 \\
\hline 2 & 20 & $128 \times 128 \times 512$ & 217.2 \\
\hline
\end{tabular}

Table 3.6: The current at the beam center after traveling ten lattice half periods, for the 5 ns test beam, as calculated by WARP3d using different numerical parameters.

base line case. These simulation parameters were varied in order to guarantee convergence.

Table 3.6 lists the current at the beam center as calculated by WARP3d after ten lattice half periods. Comparison with table 3.5 shows that the difference between Hermes and WARP3d in the final current at the beam center is about 3\%. Figure 3.10 shows the current profile after ten lattice half periods as calculated by WARP3d, by Hermes, and by the $g$-factor model.

\section{The Cause of Slice Overtaking}

Both CIRCE and Hermes suffer from slice overtaking, which is unphysical in the Lagrangian fluid model on which CIRCE and Hermes are based. The more accurate calculation of the longitudinal electric field in the Hermes model does not prevent slice overtaking to occur in all cases. In this section, we derive an analytic expression for the longitudinal electric field in the Hermes model to find the cause of slice overtaking.

In the Hermes model, for the calculation of the longitudinal electric field the beam is assumed to be circular transversely instead of elliptical. The system is then cylindrically 


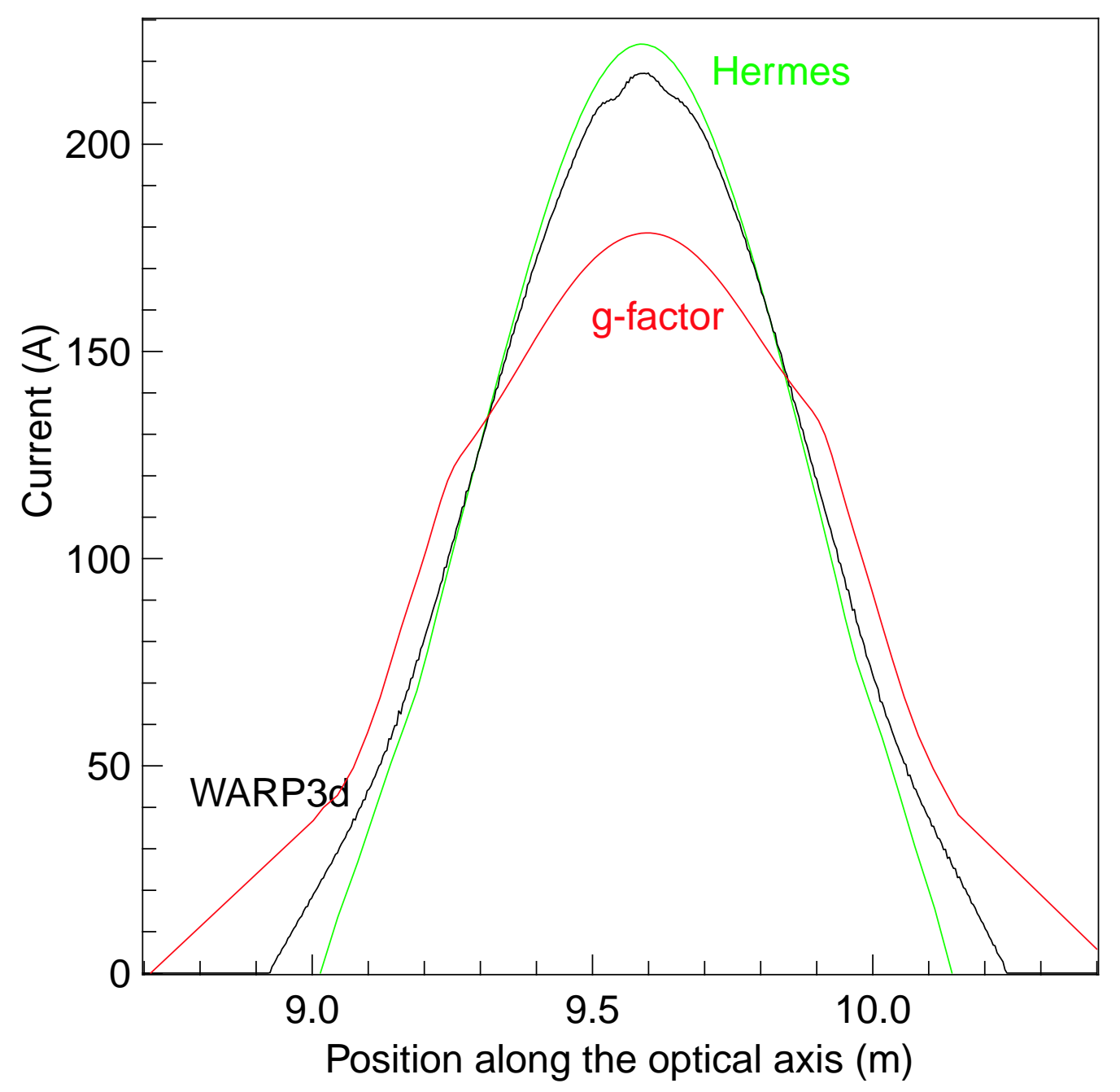

Figure 3.10: The beam current profile for an initially $5 \mathrm{~ns}$ beam of $937.5 \mathrm{~A}$ after traveling ten lattice half periods, as calculated by WARP3d, by Hermes, and by the $g$-factor model. 
symmetric, for which we can write Poisson's equation as

$$
\frac{1}{r} \frac{\partial}{\partial r}\left(r \frac{\partial \phi}{\partial r}\right)+\frac{\partial^{2} \phi}{\partial z^{2}}=-\frac{\rho}{\epsilon_{0}}
$$

The solution to this equation can be written in terms of a Fourier-Bessel expansion:

$$
\phi(r, z)=\sum_{n=1}^{\infty} f_{n}(z) J_{0}\left(\frac{x_{n} r}{R}\right)
$$

in which $J_{0}$ is the Bessel function of order zero, $x_{n}$ is the $n^{\text {th }}$ zero of $J_{0}, R$ is the pipe radius, and $f_{n}(z)$ is a set of functions to be determined [54]. Substitution of this expansion into Poisson's equation gives

$$
\sum_{n=1}^{\infty}\left[f_{n}^{\prime \prime}(z)-f_{n}(z)\left(\frac{x_{n}}{R}\right)^{2}\right] J_{0}\left(\frac{x_{n} r}{R}\right)=-\frac{\rho}{\epsilon_{0}}
$$

We can then use the identity [54]

$$
\int_{0}^{R} r J_{0}\left(\frac{x_{n} r}{R}\right) J_{0}\left(\frac{x_{m} r}{R}\right) \mathrm{d} r=\frac{1}{2}\left[J_{1}\left(x_{n}\right)\right]^{2} \delta_{n m}
$$

in which $J_{1}$ is the Bessel function of first order, to find an ordinary differential equation for the functions $f_{n}$ :

$$
f_{n}^{\prime \prime}(z)-f_{n}(z)\left(\frac{x_{n}}{R}\right)^{2}=-\frac{2}{\epsilon_{0} R^{2}} \frac{1}{\left[J_{1}\left(x_{n}\right)\right]^{2}} \int_{0}^{R} \rho r J_{0}\left(\frac{x_{n} r}{R}\right) \mathrm{d} r
$$

In the Hermes model, the charge density is uniform transversely up to the beam radius $a(z)$. We can then evaluate the integral on the right hand side of this equation, using [39]

$$
\int_{0}^{x} t J_{0}(t) \mathrm{d} t=x J_{1}(x)
$$

to find

$$
f_{n}^{\prime \prime}(z)-f_{n}(z)\left(\frac{x_{n}}{R}\right)^{2}=-\frac{2}{\epsilon_{0}} \frac{\lambda(z)}{\pi a(z)^{2}} \frac{1}{\left[x_{n} J_{1}\left(x_{n}\right)\right]^{2}} \frac{a(z) x_{n}}{R} J_{1}\left(\frac{x_{n} a(z)}{R}\right) .
$$


Now we define the functions $g_{n}(z) \equiv-f_{n}^{\prime}(z)$, such that the longitudinal electric field can be written as

$$
E_{z}(r, z)=\sum_{n=1}^{\infty} g_{n}(z) J_{0}\left(\frac{x_{n} r}{R}\right) .
$$

The functions $g_{n}(z)$ satisfy the ordinary differential equation

$$
g_{n}^{\prime \prime}(z)-g_{n}(z)\left(\frac{x_{n}}{R}\right)^{2}=\frac{2}{\epsilon_{0} R} \frac{1}{x_{n}\left[J_{1}\left(x_{n}\right)\right]^{2}} \frac{\partial}{\partial z}\left[\frac{\lambda(z)}{\pi a(z)} J_{1}\left(\frac{x_{n} a(z)}{R}\right)\right]
$$

We can solve for $g_{n}(z)$ in terms of the line charge density $\lambda(z)$ and the beam radius $a(z)$ as $[88]$

$$
g_{n}(z)=\frac{x_{n}}{\pi \epsilon_{0} R} \frac{1}{\left[x_{n} J_{1}\left(x_{n}\right)\right]^{2}} \int_{-\infty}^{+\infty} \operatorname{sign}\left(z-z^{\prime}\right) \exp \left(-\frac{x_{n}}{R}\left|z-z^{\prime}\right|\right) J_{1}\left(\frac{x_{n} a\left(z^{\prime}\right)}{R}\right) \frac{\lambda\left(z^{\prime}\right)}{a\left(z^{\prime}\right)} \mathrm{d} z^{\prime}
$$

using the boundary condition that $g_{n}(z) \longrightarrow 0$ for $|z| \longrightarrow \infty$. To find the longitudinal electric field on a slice boundary, we average over the area of the slice boundary using equation (3.22) and sum over the Fourier-Bessel components:

$$
\begin{aligned}
E_{z}(z) & =\sum_{n=1}^{\infty} \frac{1}{\pi a(z)^{2}} \int_{0}^{a(z)} g_{n}(z) J_{0}\left(\frac{x_{n} r}{R}\right) 2 \pi r \mathrm{~d} r \\
& =\sum_{n=1}^{\infty} g_{n}(z) \frac{2 R}{x_{n} a(z)} J_{1}\left(\frac{x_{n} a(z)}{R}\right) .
\end{aligned}
$$

Substituting our solution for $g_{n}(z)$, we find

$$
\begin{gathered}
E_{z}(z)=\frac{2}{\pi \epsilon_{0} a(z)} \sum_{n=1}^{\infty} \frac{1}{\left[x_{n} J_{1}\left(x_{n}\right)\right]^{2}} J_{1}\left(\frac{x_{n} a(z)}{R}\right) \\
\int_{-\infty}^{\infty} \exp \left(-\frac{x_{n}}{R}\left|z-z^{\prime}\right|\right) J_{1}\left(\frac{x_{n} a\left(z^{\prime}\right)}{R}\right) \frac{\lambda\left(z^{\prime}\right)}{a\left(z^{\prime}\right)} \mathrm{d} z^{\prime} .
\end{gathered}
$$

For a slice boundary $i$ at position $z_{i}$, we can rewrite this as

$$
E_{i}=\frac{2}{\pi \epsilon_{0} R^{2}} \sum_{n=1}^{\infty} \frac{1}{\left[J_{1}\left(x_{n}\right)\right]^{2}} \frac{J_{1}\left(x_{n} a_{i} / R\right)}{x_{n} a_{i} / R}\left(s_{i}^{\mathrm{L}}-s_{i}^{\mathrm{R}}\right)
$$


in which $s_{i}^{\mathrm{L}}$ and $s_{i}^{\mathrm{R}}$ are defined as

$$
\begin{aligned}
s_{i}^{\mathrm{L}} & \equiv \int_{-\infty}^{z_{i}} \exp \left(\frac{x_{n}}{R}\left(z^{\prime}-z_{i}\right)\right) \frac{J_{1}\left(x_{n} a\left(z^{\prime}\right) / R\right)}{x_{n} a\left(z^{\prime}\right) / R} \mathrm{~d} Q\left(z^{\prime}\right), \\
s_{i}^{\mathrm{R}} & \equiv \int_{z_{i}}^{\infty} \exp \left(\frac{x_{n}}{R}\left(z_{i}-z^{\prime}\right)\right) \frac{J_{1}\left(x_{n} a\left(z^{\prime}\right) / R\right)}{x_{n} a\left(z^{\prime}\right) / R} \mathrm{~d} Q\left(z^{\prime}\right) .
\end{aligned}
$$

We can express $s_{i}^{\mathrm{L}}$ and $s_{i}^{\mathrm{R}}$ in terms of recursion relations:

$$
\begin{aligned}
s_{i+1}^{\mathrm{L}} & =\exp \left(\frac{x_{n}}{R}\left(z_{i}-z_{i+1}\right)\right) s_{i}^{\mathrm{L}} \\
& +\int_{z_{i}}^{z_{i+1}} \exp \left(\frac{x_{n}}{R}\left(z^{\prime}-z_{i+1}\right)\right) \frac{J_{1}\left(x_{n} a\left(z^{\prime}\right) / R\right)}{x_{n} a\left(z^{\prime}\right) / R} \mathrm{~d} Q\left(z^{\prime}\right) \\
s_{i-1}^{\mathrm{R}} & =\exp \left(\frac{x_{n}}{R}\left(z_{i-1}-z_{i}\right)\right) s_{i}^{\mathrm{R}} \\
& +\int_{z_{i-1}}^{z_{i}} \exp \left(\frac{x_{n}}{R}\left(z_{i-1}-z^{\prime}\right)\right) \frac{J_{1}\left(x_{n} a\left(z^{\prime}\right) / R\right)}{x_{n} a\left(z^{\prime}\right) / R} \mathrm{~d} Q\left(z^{\prime}\right)
\end{aligned}
$$

using $s_{0}^{\mathrm{L}}=0$ and $s_{N}^{\mathrm{R}}=0$ as the starting values for the recursion. This expression for the longitudinal electric field has been implemented in Hermes as an alternative to the field solver based on WARPrz, described above. The direction of the recursion in equations (3.33), (3.34) was chosen such as to avoid numerical overflow due to large exponential factors. The integrals in the recursion relations (3.33), (3.34) were evaluated by taken the line charge density (given by $\left.\mathrm{d} Q\left(z^{\prime}\right)=\lambda\left(z^{\prime}\right) \mathrm{d} z^{\prime}\right)$ to be uniform within each slice, and the factor $\frac{J_{1}\left(x_{n} a\left(z^{\prime}\right) / R\right)}{x_{n} a\left(z^{\prime}\right) / R}$ to vary linearly from one slice boundary to the next. The integrals in the recursion relations $(3.33),(3.34)$ can then be evaluated analytically, which results in an expression that resembles the trapezoidal rule. Applying the trapezoidal rule directly to the integrals in the recursion relations gives poor results, as the exponential fall-off cannot be represented well by a linear function, particularly for Bessel modes with large $x_{n}$.

The Fourier-Bessel model has shown agreement with the results using WARPrz's field solver. However, since we assume in the Fourier-Bessel model that the line charge 
density is uniform within each slice, typically we need a much larger number of slices to reach convergence in the Fourier-Bessel model. For the 5 ns test beam discussed above, the Fourier-Bessel model implemented in Hermes, using 400 slices with 100 steps per lattice half period and 128 terms in the Fourier-Bessel expansion, the current at the beam center after traveling ten lattice half periods was calculated to be $223.65 \mathrm{~A}$, compared to $224.15 \mathrm{~A}$ using Hermes with WARPrz's field solver (table 3.5).

We can now consider the electric field on a slice boundary $i$ as it approaches slice boundary $i+1$. Let the two slice boundaries have an equal radius $a$. From equations (3.30) and (3.33), we find that the electric field due to the charge $\Delta Q$ between them, summed over the Bessel-Fourier components $n$ is

$$
\Delta E_{i}(z)=\frac{2 \Delta Q}{\pi \epsilon_{0} R^{2}} \sum_{n=1}^{\infty}\left[\frac{J_{1}\left(x_{n} a / R\right)}{J_{1}\left(x_{n}\right) x_{n} a / R}\right]^{2} .
$$

For $a / R \longrightarrow 0$, the sum diverges, and we find $\Delta E_{i}(z) \longrightarrow \infty$. In this limit, the slice reduces to a point charge in the center of a conducting pipe, for which the repulsive force diverges as we approach it. For $a / R=1$, we use [98]

$$
\sum_{n=1}^{\infty}\left(\frac{1}{x_{n}}\right)^{2}=\frac{1}{4}
$$

to find $\Delta E_{i}(z)=\Delta Q /\left(2 \pi \epsilon_{0} R^{2}\right)$. For $a / R$ between zero and one, the sum was evaluated numerically. Figure 3.11 shows the repulsive electric field that was found from equation (3.35) for a slice compressed to zero length, as a function of the beam radius $a$ divided by the pipe radius $R$. For a non-zero beam radius, the repulsive electric field attains a finite value. This means that if slice boundary $i$ approaches slice boundary $i+1$ with a sufficiently large velocity, the repelling force on slice boundary $i$ will not be strong enough to stop it. Slice overtaking will then occur. 


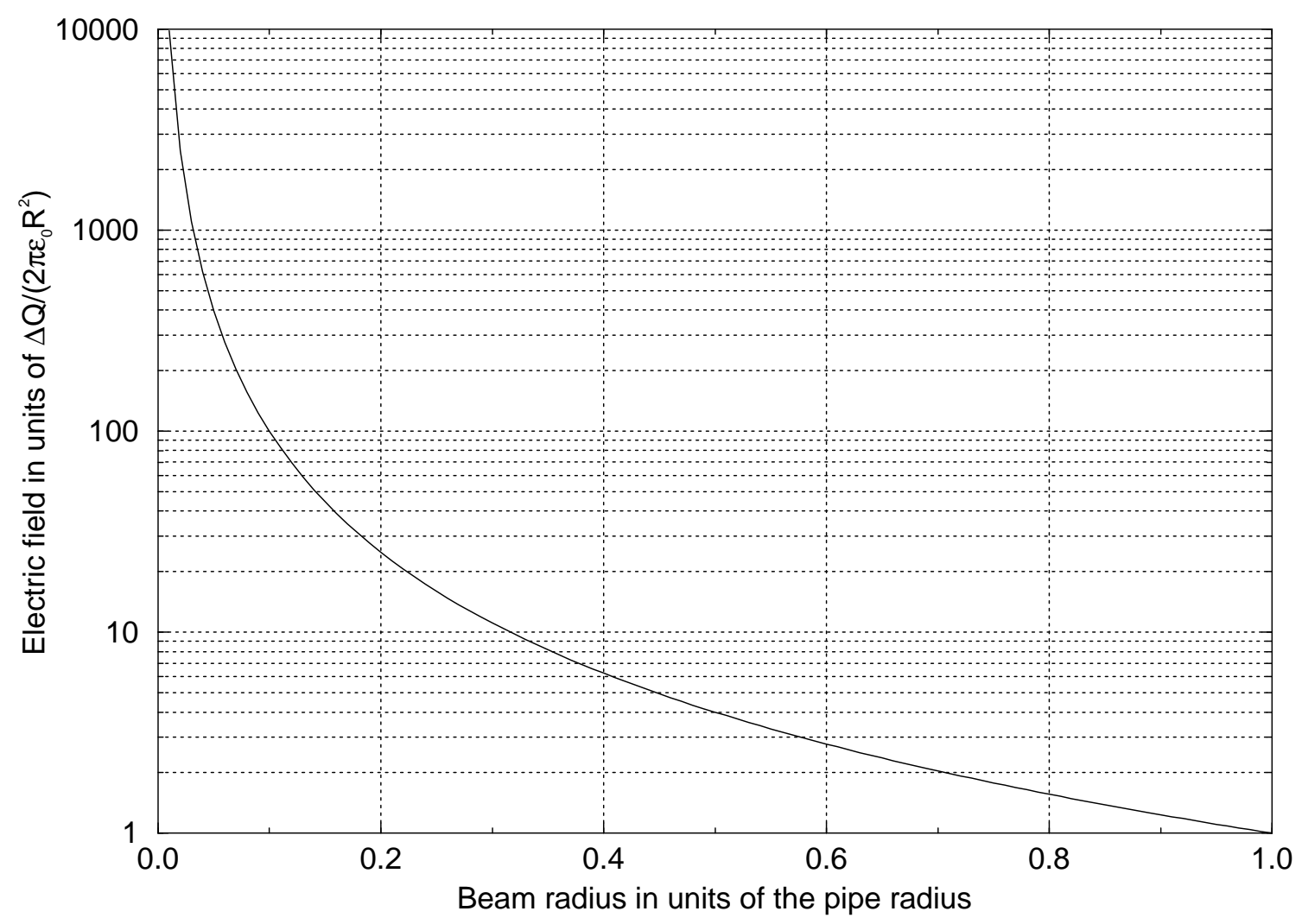

Figure 3.11: The repulsive electric field at the surface of a slice, compressed to zero length, as a function of the beam radius of the slice.

We conclude that the fluid model on which CIRCE and Hermes are based, do not exclude the possibility of slice overtaking. Slice overtaking may be prevented by adding a pressure term due to the longitudinal emittance of the beam to the equation of motion of the slice boundaries, since the repelling force would then be unbounded as two slice boundaries approach each other. However, the longitudinal emittance that exists in beams relevant for heavy ion inertial fusion is typically too low to satisfactorily solve the slice overtaking problem. Before pressure would stop two slice boundaries approaching each other, the slice would be compressed to such a degree that the current in that slice would become enormous. This is not what we expect physically to happen. Instead, it should be viewed 
as an indication that kinetic effects are important for the system under consideration, and that the fluid model on which CIRCE and Hermes are based is inadequate. 


\section{Chapter 4}

\section{The IRE Drift Compression}

\section{Section}

As we saw in the discussion of the $g$-factor model in section 3.2.3, the longitudinal electric field in a charged particle beam is shielded by the conducting pipe in which the beam travels. The shielding effect depends on the beam and pipe radii, as reflected in equations (3.7) and (3.9) in the $g$-factor model. Therefore, the longitudinal dynamics of the beam depends on the transverse dynamics. Inversely, the transverse dynamics of the beam depends on the perveance and hence on the current, which depends on the longitudinal compression or expansion that the beam has undergone. So the longitudinal and transverse dynamics of a space-charge dominated beam are coupled. In order to design a drift compression section, in which the current changes continuously, we need to resort to iteration over small longitudinal distances. On each iteration, small changes are made to the lattice properties, until the transverse and longitudinal dynamics of the beam have converged. 


\subsection{Designing a Drift Compression Section}

The design of a drift compression section for the IRE is based on the standard IRE parameters given in table 1.2. At the end of the accelerator, the beam has a kinetic energy of $200 \mathrm{MeV}$. No further acceleration takes place in the drift compression section. Each beam has a charge of $4.6875 \mu \mathrm{C}$, with a pulse duration of $336 \mathrm{~ns}$ at the end of the accelerator. Our aim is to reduce the pulse duration to about $10 \mathrm{~ns}$ to $20 \mathrm{~ns}$ at the end of the drift compression section, based on target temperature considerations [7], which corresponds to an average beam current of $937.5 \mathrm{~A}$ to $468.75 \mathrm{~A}$ for a flat current profile. As we showed in section 3.2.4, the $g$-factor model is inaccurate in this regime. Therefore, the Hermes model was used to design a drift compression section for the IRE.

Designing a drift compression section consists of one backward and one forward

Hermes run. In the backward run, the drift compression section is set up starting from a desired final beam pulse at the end of drift compression. We choose a beam duration we would like to achieve there, and we find the corresponding final current. We assume the current profile to be a flat-top, with $25 \%$ parabolic fall-offs on each side. At final focus, the occupancy $\eta$ was chosen to be $65 \%$, which is near the practically achievable limit. For the designs presented here, the occupancy was held constant in the drift compression section, although that is not a necessary design requirement. The undepressed tune was chosen to be $72^{\circ}$, which is low enough for the beam to be stable. The beam radius at the entrance of the final focus section was chosen to be $6 \mathrm{~cm}$, since we need a fairly large beam radius in the final focus system in order to be able to focus the beam on target. These numerical values allow us to calculate the perveance at final focus and the lattice half period that we 
would need for a transport lattice from the approximate relations described in section 2.3.1. We can then set up a transport lattice in WARP and use WARP's envelope solver to find the magnetic field gradient in the quadrupoles to achieve a tune of $72^{\circ}$.

Next, we set up a large $(\gtrsim 300)$ number of lattice half periods of this transport lattice. We then initialize a Hermes beam in the last couple of half periods, making sure the entire beam is covered by the lattice. The beam is set up such that the leftmost slice boundary is at the beginning of a lattice half period, and the beam radii of all the slice boundaries are matched to the transport lattice.

The beam is then simulated backward in time, from the end of the drift compression to the beginning. In this backward run, the current decreases. In order to set up the lattice, we need to iterate over each lattice half period. First, we save the parameters of the Hermes beam at the beginning of the iteration step. We then run Hermes backwards in time, each time step being negative, until the center of the beam reaches the center of the preceding lattice half period. Since the beam has expanded longitudinally during this time step, the current at the beam center has decreased. Therefore, the lattice half period and focusing force of this half period do not exactly correspond to the actual current at the beam center. We can calculate what the half period length and the focusing force should be for a matched beam using the approximate formulas described in section 2.3.1. We then adjust the previous half period, and all other half periods to the left of it, such that its length and quadrupole strength correspond to the current we found. The Hermes beam is then reloaded from its saved values, and Hermes is again run through this half period. Once the difference between the calculated half period length and the actual half period 
length is smaller than a given error limit, our solution has converged and the procedure is repeated for the next lattice half period. For the calculations presented here, an error limit of $1 . \cdot 10^{-9}$ was chosen. The iteration converges quickly, typically taking about six steps for each half period. This procedure is continued until the current at the beam center has reduced to the level at the end of the accelerator. The quadrupoles that have not been used are then removed from the simulation.

In addition to keeping the beam matched, we also need to reduce the beam radius from its value at the final focus to its value at the end of the accelerator. To do this, we need to describe how we would like the beam radius to vary as a function of $z$. For the simulations presented here, we chose a smooth falloff given by

$$
a(z)= \begin{cases}a_{\mathrm{acc}}+\frac{1}{2}\left(a_{\mathrm{ff}}-a_{\mathrm{acc}}\right)\left[1+\tanh \left(\cot \left(\pi \frac{L_{\mathrm{dc}}-z}{L_{\mathrm{inc}}}\right)\right)\right] & \text { for } z>L_{\mathrm{dc}}-L_{\mathrm{inc}} \\ a_{\mathrm{acc}} & \text { for } z<L_{\mathrm{dc}}-L_{\mathrm{inc}}\end{cases}
$$

in which $a_{\text {acc }}$ is the average beam radius at the end of the accelerator, as determined by the current IRE design, $a_{\mathrm{ff}}$ is the average beam radius that we have chosen at the beginning of the final focus section, $z$ is the distance from the beginning of the drift compression section, $L_{\mathrm{dc}}$ is the total length of the drift compression system, and $L_{\text {inc }}$ is the distance over which we want the beam radius to increase from $a_{\text {acc }}$ to $a_{\mathrm{fff}}$. Other choices, such as a simple linear falloff, may cause mismatches to occur near discontinuities in $a(z)$ or its derivatives.

For one iteration step, we first find the current of the beam center in the middle of the half period from the Hermes run of the previous iteration step. We calculate the perveance from the current, and evaluate equation (4.1) to find the desired average beam radius in the middle of the half period. We then substitute the perveance and average beam radius into equation (2.60) to find the half period length for the next iteration step. 
Using this new value in equation (2.61) gives us the corresponding focusing strength of the quadrupole. The aperture is chosen based on the heuristic

$$
\text { aperture }=1.25 \times \text { maximum beam radius }+5 \mathrm{~mm} \text {, }
$$

allowing a $5 \mathrm{~mm}$ clearance for misalignments [28]. Setting up the aperture correctly is crucial, since the magnitude of the longitudinal electric field depends on the amount of shielding by the aperture. The Python script to set up the IRE drift compression section is given in Appendix A.1.

As an example, consider a beam with a 20 ns final pulse duration, using $L_{\text {inc }}=$ $150 \mathrm{~m}$ in equation (4.1). This distance was chosen to illustrate the important issue of rematching at the beginning of drift compression, discussed below. More realistic designs for an IRE drift compression system, such as those described in section 4.2, would use a shorter distance $L_{\text {inc }}$.

Figure 4.1 shows the beam current profile as a function of position at the end of drift compression for this example, whereas figure 4.2 shows the beam radius at the center of the beam, together with the aperture. Although the procedure outlined above keeps the center of the beam approximately matched, other parts of the beam may become mismatched, particularly near the beam ends where the current is changing rapidly. Figure 4.3 shows the current of the various slice boundaries as a function of distance along the drift compression system. Mismatches will occur if the current changes rapidly enough to prevent the beam from adjusting itself adiabatically. In the present case, the distance over which the current changes is comparable to the period of the betatron oscillation $(8.24 \mathrm{~m}$ for the current of the second slice boundary to increase from 16 A to 32 A compared to a betatron 
period of $9.66 \mathrm{~m}$ ), resulting in a severe mismatch near the ends of the beam, as shown in figure 4.4. The mismatch will be even worse if the final pulse duration or the distance $L_{\text {inc }}$ in equation (4.1) is reduced.

Note that the mismatch is produced at the end of the drift compression section, where the beam current changes most rapidly. In the backward run, the mismatch then persists up to the beginning of the drift compression section. These mismatches are undesirable, since they may result in an emittance increase as well as halo formation. To minimize the distance of the drift compression over which mismatches occur, we should therefore rematch all slice boundaries of the beam once the backward run reaches the beginning of the drift compression. The simulation is then run forward from the beginning of the drift compression to the end. In this forward run of the rematched beam, some mismatch will now occur near the end of drift compression. Since this mismatch will be formed fairly late, it will not affect the beam severely. We then compare the beam properties at the end of drift compression to what we intended to achieve. The RMS difference between the final and the originally specified current profile provides a quantitative measure of their resemblance.

Figure 4.5 shows the beam radius of the second slice boundary if we rematch at the beginning of the drift compression. Now the beam remains matched for most of the drift compression, although a small mismatch develops near the end.

Rematching the beam does not significantly affect the longitudinal dynamics. Figure 4.6 shows the current profile at the end of drift compression, if the beam is rematched at the beginning of drift compression. The current profile is close to the originally specified current profile, as shown by the RMS current deviation being only $0.65 \%$. 


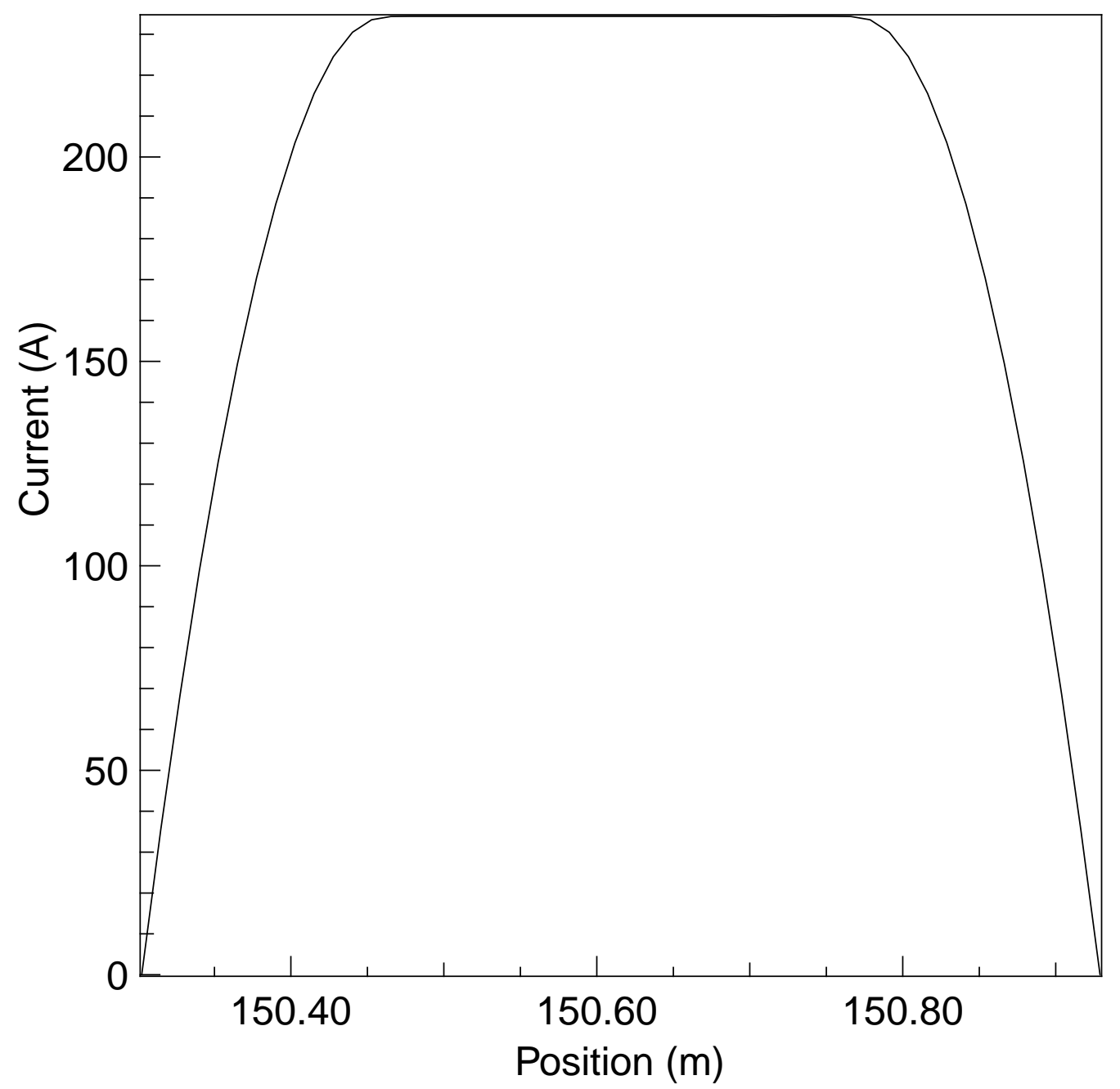

Figure 4.1: The current profile as a function of position, for a 20 ns final pulse duration, if the beam is not rematched at the beginning of drift compression. For this calculation, 50 slices were used on a $64 \times 128 \mathrm{RZ}$ grid with a $3 \mathrm{~cm}$ step size. 


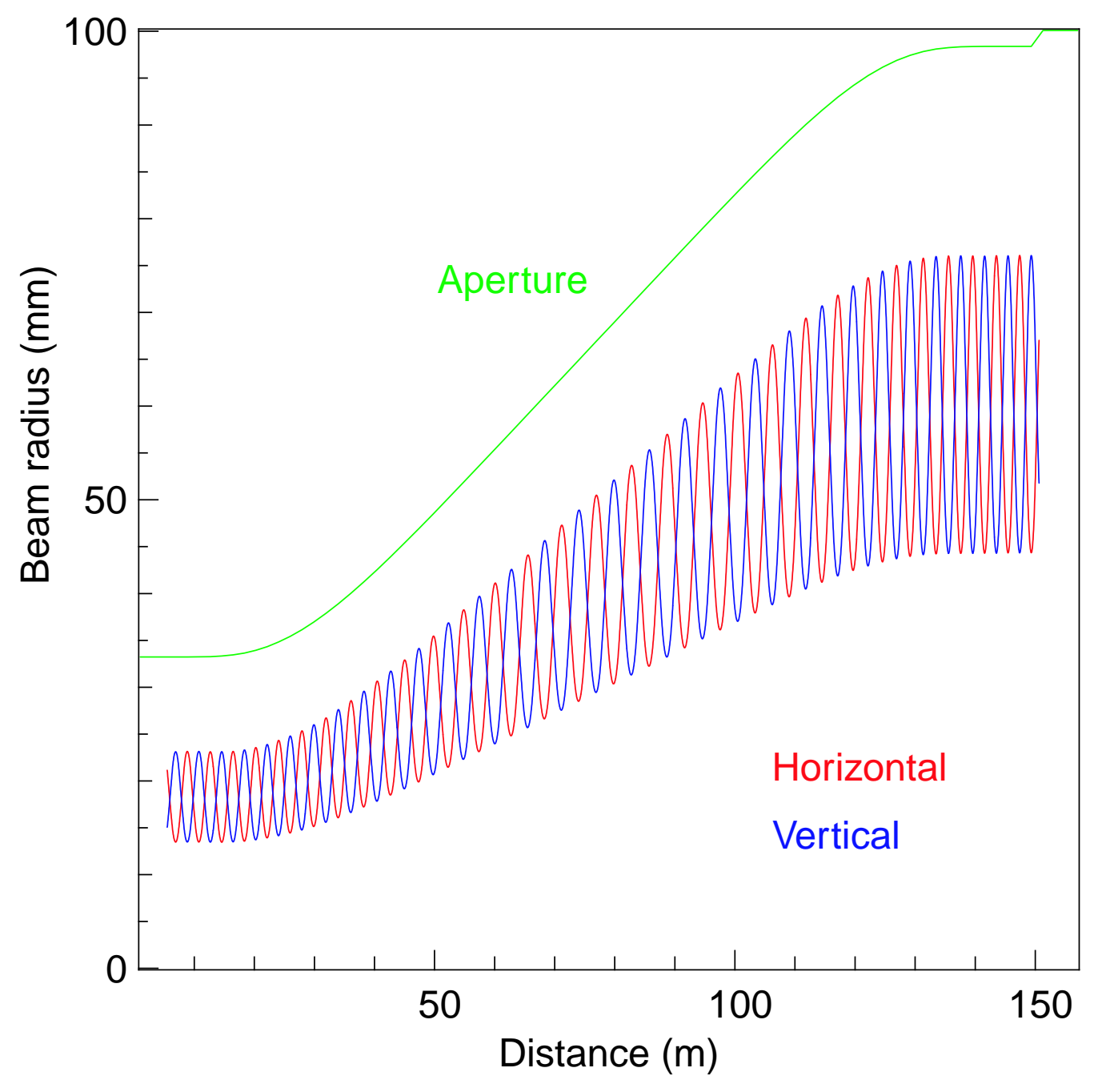

Figure 4.2: The transverse beam envelope for the beam center, for a $20 \mathrm{~ns}$ final pulse duration, if the beam is not rematched at the beginning of drift compression. For this calculation, 50 slices were used on a $64 \times 128 \mathrm{RZ}$ grid with a $3 \mathrm{~cm}$ step size. 


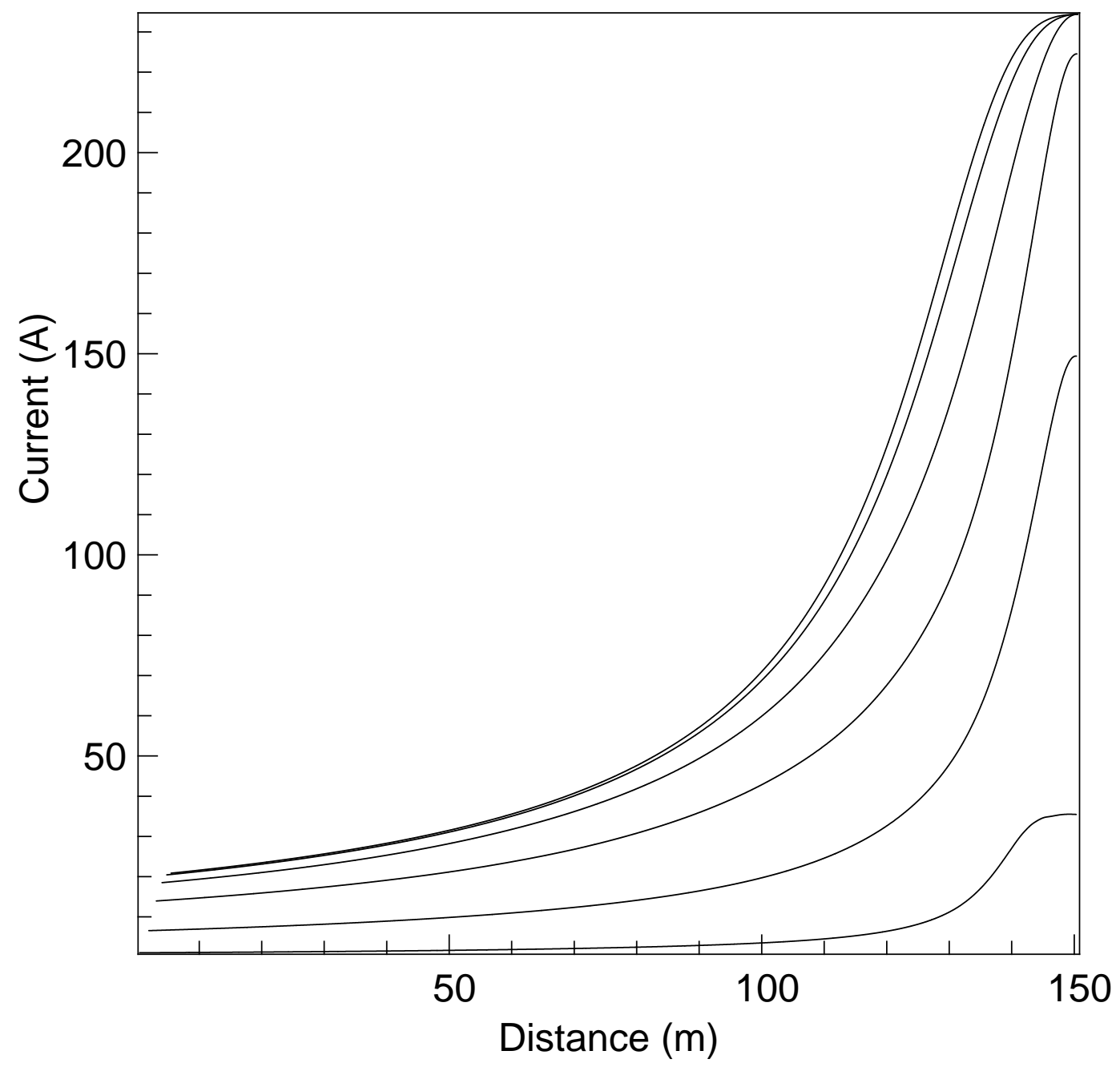

Figure 4.3: The current for different slices of the beam for a 20 ns final pulse duration, if the beam is not rematched at the beginning of drift compression. For this calculation, 50 slices were used on a $64 \times 128 \mathrm{RZ}$ grid with a $3 \mathrm{~cm}$ step size. 


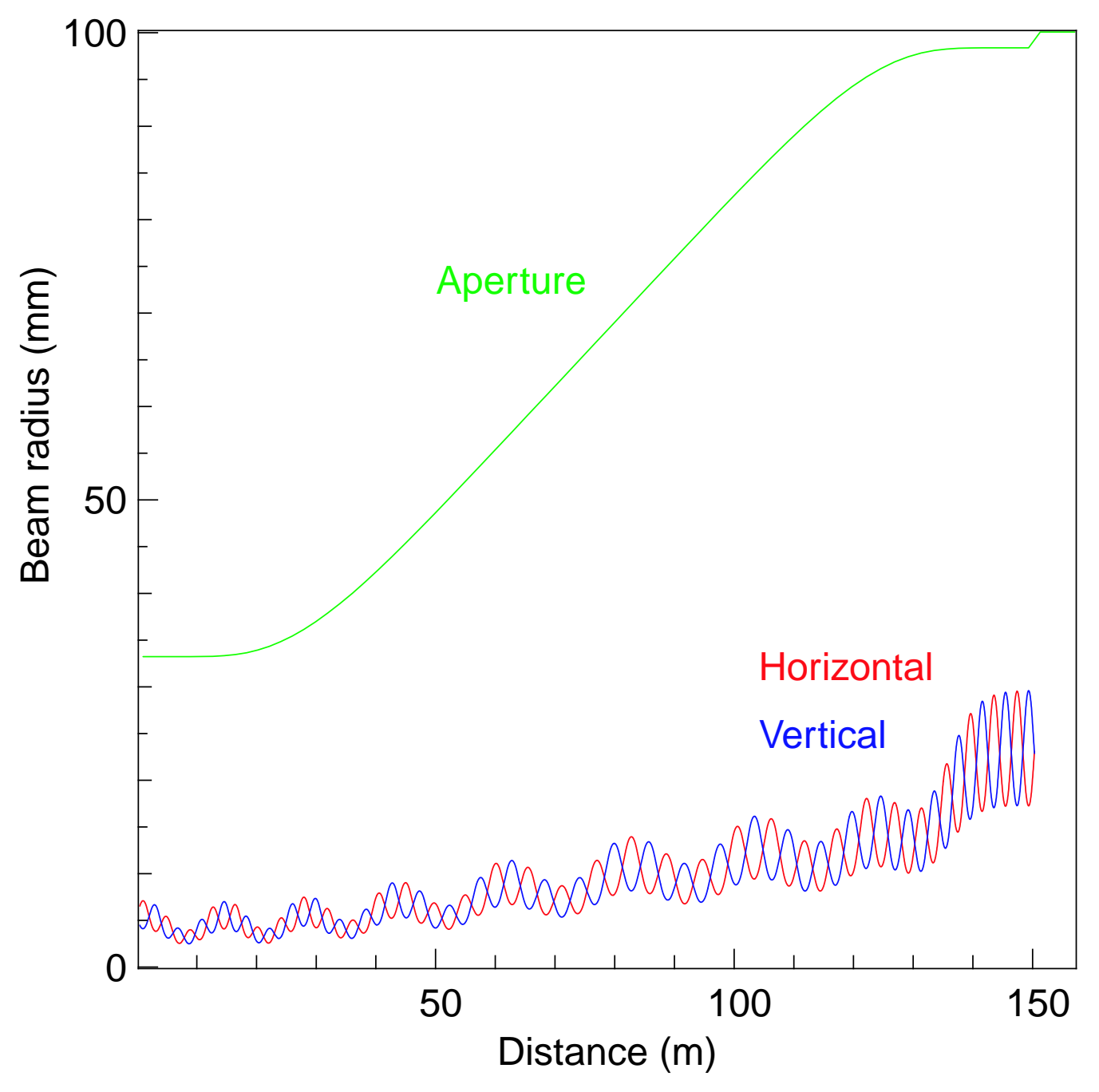

Figure 4.4: The transverse beam envelope for the second slice boundary of the beam, for a $20 \mathrm{~ns}$ final pulse length, if the beam is not rematched at the beginning of drift compression. For this calculation, 50 slices were used on a $64 \times 128 \mathrm{RZ}$ grid with a $3 \mathrm{~cm}$ step size. 


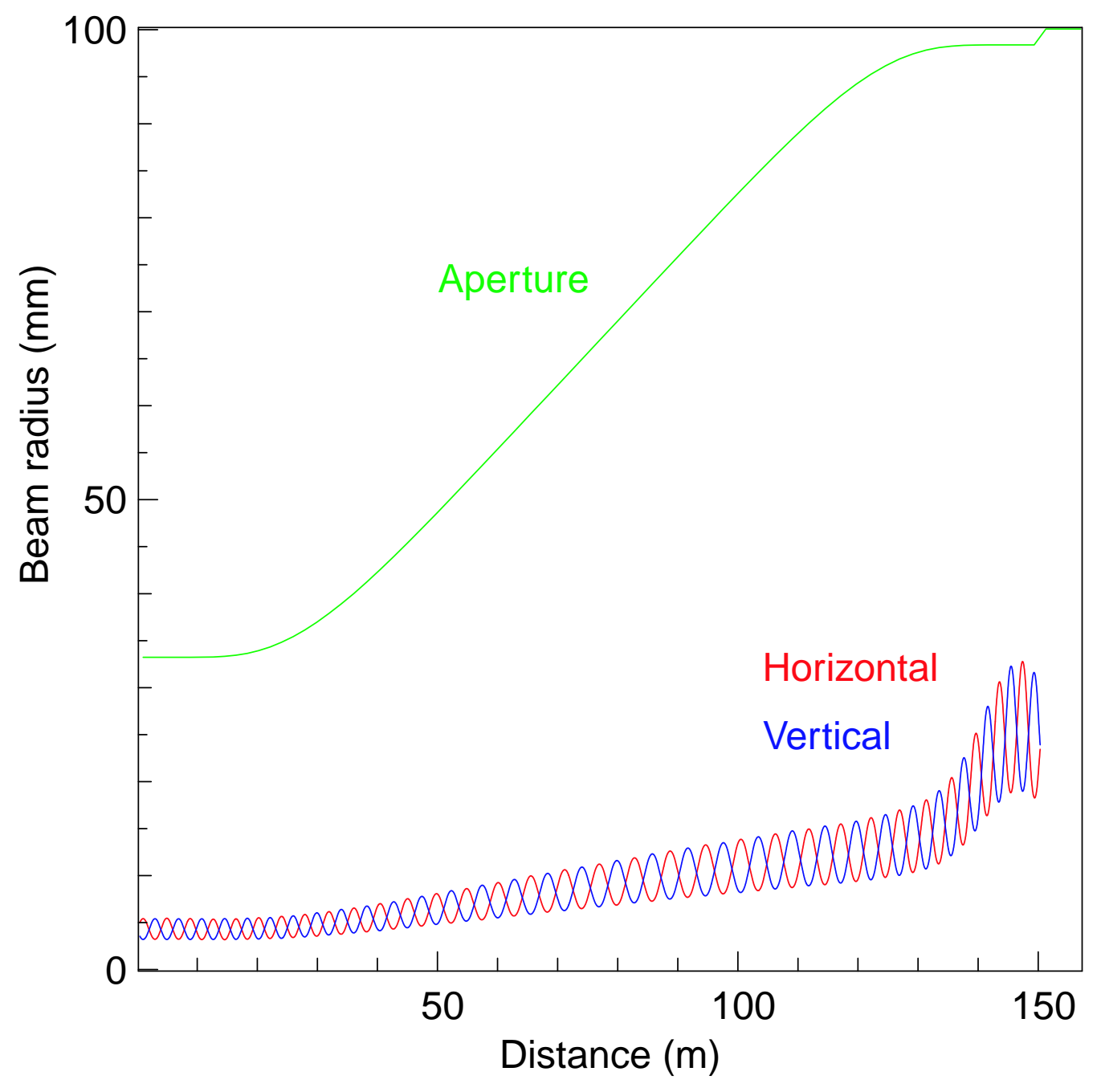

Figure 4.5: The transverse beam envelope for the second slice boundary of the beam, for a 20 ns final pulse duration, if the beam is rematched at the beginning of drift compression. For this calculation, 50 slices were used on a $64 \times 128 \mathrm{RZ}$ grid with a $3 \mathrm{~cm}$ step size. 


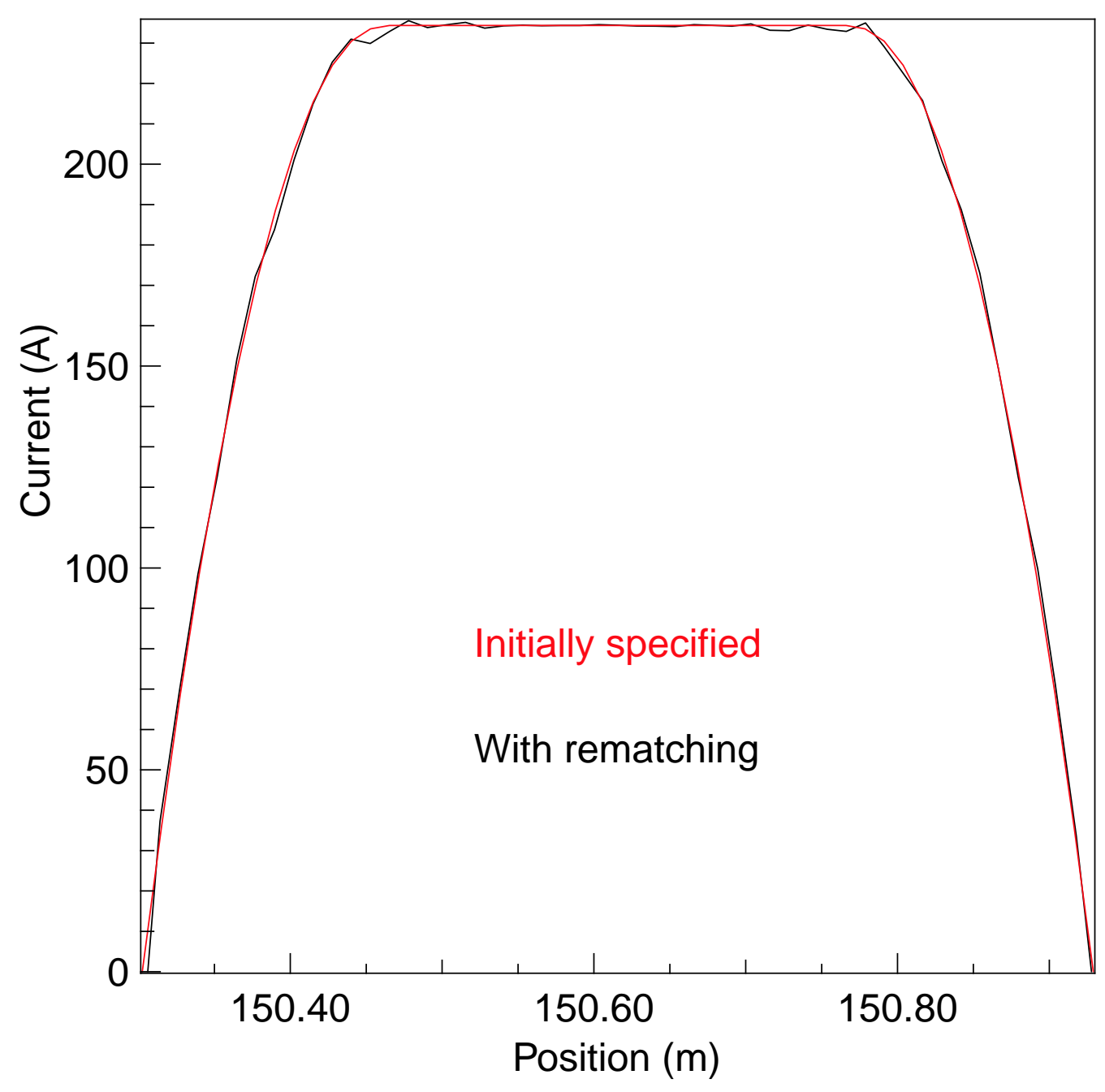

Figure 4.6: The current profile as a function of position, for a 20 ns final pulse duration, if the beam is rematched at the beginning of drift compression. The change in mean beam radius took place over $150 \mathrm{~m}$. For this calculation, 50 slices were used on a $64 \times 128 \mathrm{RZ}$ grid with a $3 \mathrm{~cm}$ step size. 


\subsection{IRE Drift Compression Designs}

In practical designs for an IRE drift compression section, we would like to keep the beam radius small for most of the drift compression. In order to check the feasibility of this, several drift compression sections were designed for final pulse durations of $20 \mathrm{~ns}$, $15 \mathrm{~ns}$, and $10 \mathrm{~ns}$, whereas in equation (4.1) the distance $L_{\text {inc }}$ for the beam radius to increase was chosen to be 25 times the lattice half period length at final focus. The final beam pulse after rematching at the beginning of the drift compression section was then compared to the initially specified beam pulse. Table 4.1 lists the numerical parameters that were used for the Hermes calculations.

Figure 4.7 shows the final beam pulse after rematching at the beginning of drift compression, together with the initially specified final beam pulse, for final beam durations of $20 \mathrm{~ns}, 15 \mathrm{~ns}$, and $10 \mathrm{~ns}$. In all cases, the final beam pulse after rematching is close to what was initially specified. Table 4.2 lists the RMS current difference between the final beam pulse and the initially specified final beam pulse for the three cases under consideration, as well as the length of the drift compression section and the required initial velocity tilt that was found from the Hermes calculations. Figure 4.8 shows the initial velocity tilt for the final pulse durations for the $20 \mathrm{~ns}, 15 \mathrm{~ns}$, and $10 \mathrm{~ns}$ final pulse durations. Since this velocity profile is smooth, we believe that such a velocity profile is achievable in practice.

\begin{tabular}{|c|c|c|c|}
\hline Final beam duration (ns) & Number of slices & RZ grid size & Step size $(\mathrm{cm})$ \\
\hline \hline 20 & 50 & $64 \times 128$ & 3 \\
\hline 15 & 50 & $64 \times 128$ & 3 \\
\hline 10 & 50 & $64 \times 256$ & 3 \\
\hline
\end{tabular}

Table 4.1: Numerical parameters used in the Hermes calculations for the IRE drift compression designs. 


\begin{tabular}{|c|c|c|c|}
\hline $\begin{array}{c}\text { Final beam } \\
\text { duration (ns) }\end{array}$ & $\begin{array}{c}\text { Initial head-to-tail } \\
\text { velocity tilt (\%) }\end{array}$ & $\begin{array}{c}\text { Drift compression } \\
\text { length (m) }\end{array}$ & $\begin{array}{c}\text { RMS current } \\
\text { deviation (\%) }\end{array}$ \\
\hline \hline 20 & $\begin{array}{c}8.68 \\
(6.24)\end{array}$ & $\begin{array}{c}145.36 \\
(150.04)\end{array}$ & 0.97 \\
\hline \multirow{2}{*}{15} & $\begin{array}{c}9.76 \\
(7.27)\end{array}$ & $\begin{array}{c}127.98 \\
(127.77)\end{array}$ & 1.25 \\
\hline 10 & $\begin{array}{c}12.31 \\
(8.99)\end{array}$ & $\begin{array}{c}107.38 \\
(102.24)\end{array}$ & 4.50 \\
\hline
\end{tabular}

Table 4.2: Initial velocity tilt, drift compression length, and deviation from the initially specified beam current at the end of drift compression due to rematching at the beginning of drift compression, as calculated by Hermes. Values in brackets are based on the $g$-factor model (equations (4.11) and (4.12)).

The required head-to-tail velocity tilt of $8.68 \%$ to $12.31 \%$ is quite large though.

Although the final current profile at final focus is a flat-top with parabolic ends, the current profile is close to parabolic for most of the drift compression system. Figure 4.9 shows the current profile at different locations along the drift compression section for a beam with a 10 ns pulse duration at final focus. The current profile remains virtually unchanged for most of the drift compression system, except near final focus (compare to figure 4.7).

We can compare the length of the drift compression section and the required initial velocity tilt to simple estimates based on the $g$-factor model. From equations (3.1) and (3.7) we find the longitudinal equation of motion

$$
m \gamma^{3} \frac{\mathrm{d} v}{\mathrm{~d} t}=-g \frac{q e}{4 \pi \epsilon_{0} \gamma^{2}} \frac{\partial \lambda}{\partial z}
$$

For a parabolic bunch of length $\ell$ at position $z_{\mathrm{c}}$, the line charge density is

$$
\lambda=\lambda_{0} \frac{\ell_{\mathrm{i}}}{\ell}\left[1-\left(\frac{z-z_{\mathrm{c}}}{\frac{1}{2} \ell}\right)^{2}\right],
$$

in which $\lambda_{0}$ is the initial line charge density at the beam center and $\ell_{\mathrm{i}}$ is the initial bunch 

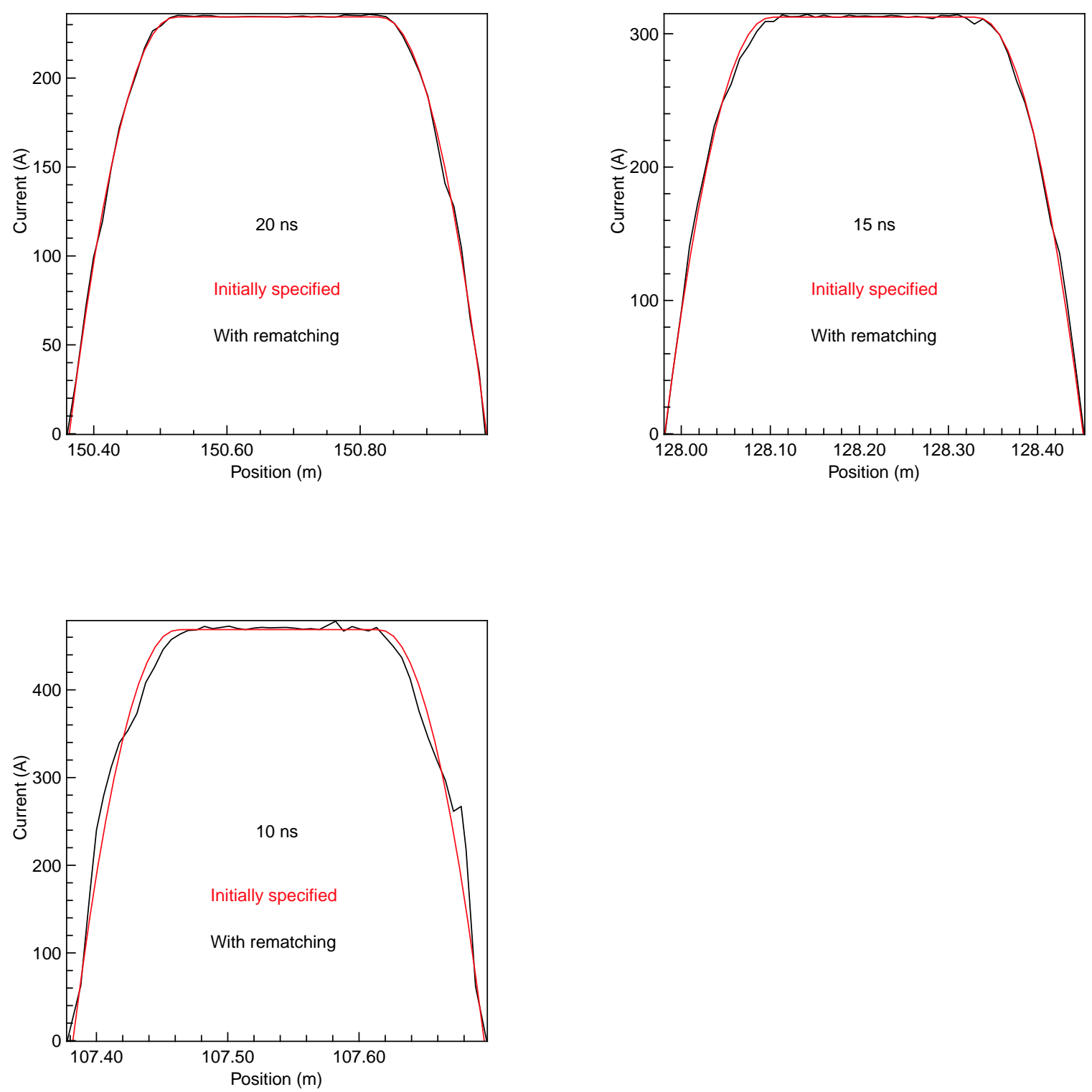

Figure 4.7: The current profile as a function of position, at the end of drift compression, for different final pulse durations, if the beam is rematched at the beginning of drift compression. The change in mean beam radius took place over 25 times the lattice half period length at the end of drift compression. 

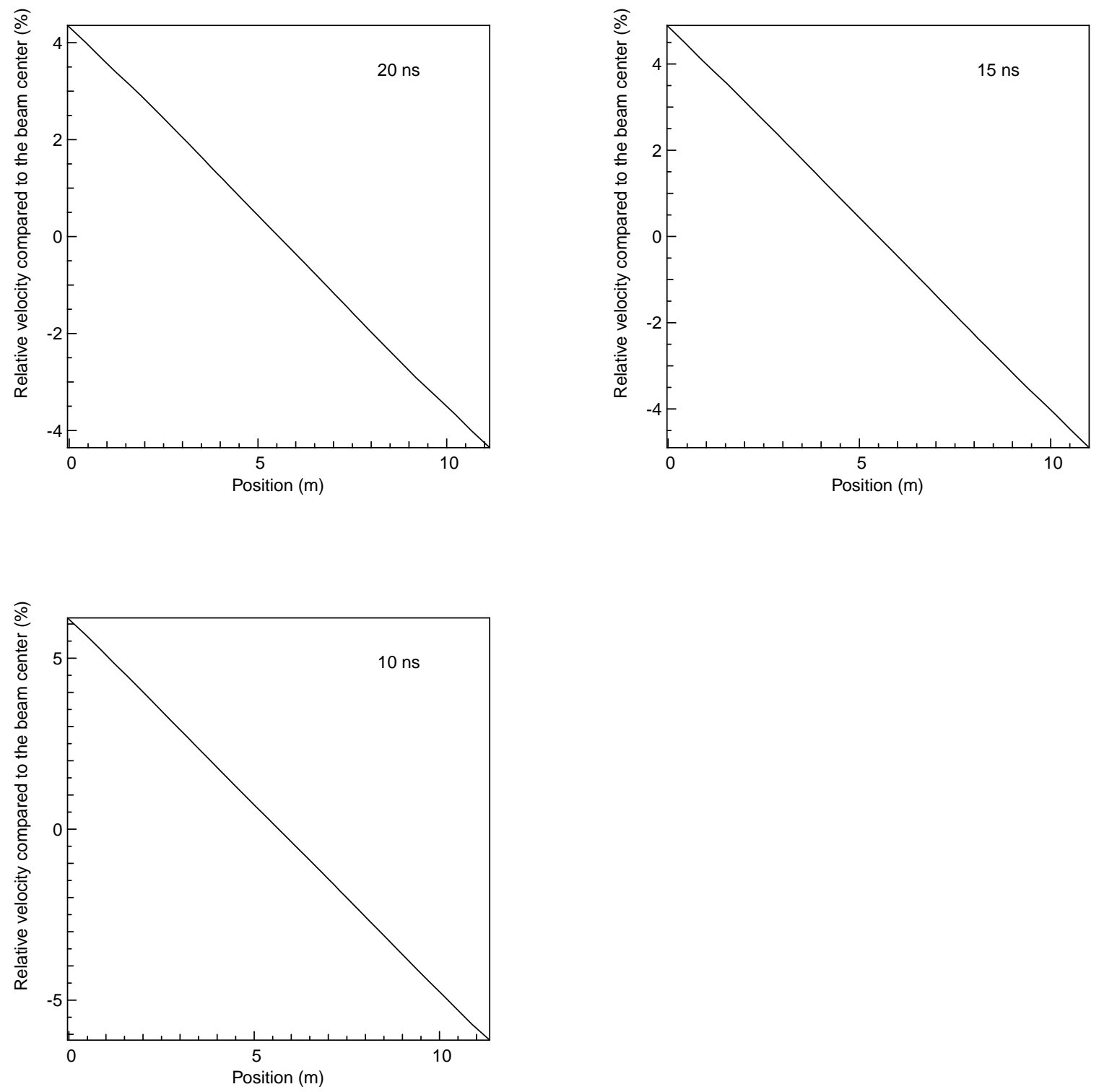

Figure 4.8: The initial velocity tilt with respect to the beam center, for $20 \mathrm{~ns}, 15 \mathrm{~ns}$, and $10 \mathrm{~ns}$ final pulse durations. 

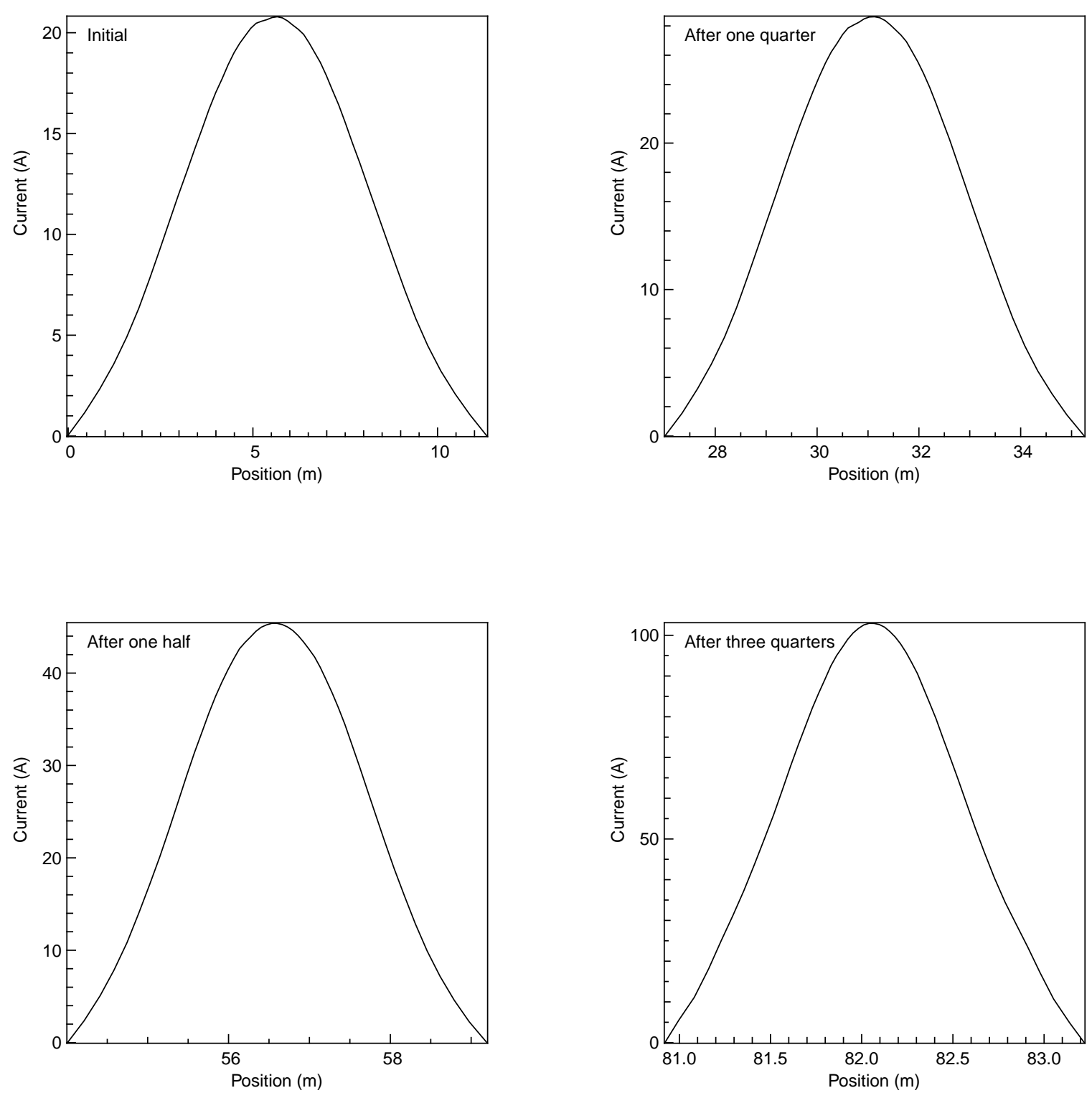

Figure 4.9: The current profile as a function of position, at various locations along the drift compression section, for a $10 \mathrm{~ns}$ final pulse duration (compare to figure 4.7, which shows the current profile at the end of drift compression). 
length. Substitution then gives

$$
\frac{\mathrm{d} v}{\mathrm{~d} t}=8 g \frac{q e}{4 \pi \epsilon_{0} m \gamma^{5}} \frac{z-z_{\mathrm{c}}}{\ell^{2}} \lambda_{0} \frac{\ell_{\mathrm{i}}}{\ell} .
$$

If we approximate $g$ to be a fixed number, instead of using equation (3.9), the right-handside is linear in $z$. A parabolic bunch will then remain parabolic during compression. By evaluating this equation at the beam ends, we find the equation of motion for the length of the beam:

$$
\begin{aligned}
\frac{\mathrm{d}^{2} \ell}{\mathrm{d} t^{2}} & =\left.\frac{\mathrm{d} v}{\mathrm{~d} t}\right|_{z-z_{\mathrm{c}}=\ell / 2}-\left.\frac{\mathrm{d} v}{\mathrm{~d} t}\right|_{z-z_{\mathrm{C}}=-\ell / 2} \\
& =8 g \frac{q e \lambda_{0}}{4 \pi \epsilon_{0} m \gamma^{5}} \frac{\ell_{\mathrm{i}}}{\ell^{2}}
\end{aligned}
$$

In terms of the initial perveance $Q_{0}$ at the beam center, we can rewrite this as

$$
\frac{\mathrm{d}^{2} \ell}{\mathrm{d} z^{2}}=\frac{4 g Q_{0}}{\gamma^{2}} \frac{\ell_{\mathrm{i}}}{\ell^{2}}
$$

using $\mathrm{d} z=v_{0} \mathrm{~d} t$, in which $v_{0}$ is the velocity of the center of the beam. Integrating this equation once gives

$$
\frac{\mathrm{d} \ell}{\mathrm{d} z}=-\sqrt{\frac{8 g Q_{0}}{\gamma^{2}} \ell_{\mathrm{i}}\left(\frac{1}{\ell_{\mathrm{f}}}-\frac{1}{\ell}\right)},
$$

using that due to the space-charge repulsion the beam compression stagnates at the end of drift compression, where the beam reaches its final bunch length $\ell_{\mathrm{f}}$. From this equation, we find the initial velocity tilt required to compress the beam to a given final bunch length:

$$
\begin{aligned}
\left.\frac{\Delta v}{v}\right|_{\mathrm{i}} & =\left.\frac{\mathrm{d} \ell / \mathrm{d} t}{\mathrm{~d} z / \mathrm{d} t}\right|_{\mathrm{i}} \\
& =\sqrt{8 g Q_{0}\left(\frac{\ell_{\mathrm{i}}}{\ell_{\mathrm{f}}}-1\right)} .
\end{aligned}
$$


By integrating equation (4.9), we find the length $L_{\mathrm{dc}}$ of the drift compression section

$$
\begin{aligned}
L_{\mathrm{dc}} & =\frac{\ell_{\mathrm{f}}}{\Delta v / v}\left[\frac{\ell_{\mathrm{i}}}{\ell_{\mathrm{f}}}-1+\sqrt{1-\frac{\ell_{\mathrm{f}}}{\ell_{\mathrm{i}}}} \operatorname{arcsinh}\left(\sqrt{\frac{\ell_{\mathrm{i}}}{\ell_{\mathrm{f}}}-1}\right)\right] \\
& \approx \frac{\ell_{\mathrm{i}}-\ell_{\mathrm{f}}}{\Delta v / v} \text { for } \ell_{\mathrm{i}} \gg \ell_{\mathrm{f}} .
\end{aligned}
$$

For the IRE design given in table 1.2, the charge per beam at the end of the accelerator is $4.6875 \mu \mathrm{C}$ at a beam duration of 335 ns. For a parabolic bunch, the current at the beam center is then 20.99 A. The backward Hermes calculation to set up the drift compression lattice continued until the calculated current at the beam center reached this level. Note that the total charge in a beam in the Hermes calculations presented above is somewhat smaller than what is given in table $1.2(3.90625 \mu \mathrm{C}$ compared to $4.6875 \mu \mathrm{C})$ due to the parabolic current fall-off near the ends of the beam. Consequently, for a fixed current level of 20.99 A at the beam center, the beam duration at the beginning of drift compression is somewhat smaller (280 ns) in the Hermes calculation. For consistency, the evaluation of equations (4.11) and (4.12) used an $\ell_{\mathrm{i}}$ corresponding to an initial beam duration of $280 \mathrm{~ns}$, whereas the perveance $Q_{0}=2.975 \times 10^{-5}$ corresponds to an initial current at the beam center of 20.99 A. For the $g$-factor, a fixed value of 1.27 was used, based on a beam radius of $17.9 \mathrm{~mm}$. This is the average beam radius at the end of the accelerator, which is maintained for most of the drift compression, until the beam expands transversely when approaching the final focus section.

We can now calculate the initial velocity tilt and length of the drift compression section using the simple $g$-factor model described above. Table 4.2 contains the initial velocity tilt and the length of the drift compression section for final pulse durations of $20 \mathrm{~ns}, 15 \mathrm{~ns}$, and $10 \mathrm{~ns}$ as estimated from equations (4.11) and (4.12), in addition to the 
Hermes results. We find that the $g$-factor model underestimates the initial tilt by about $20 \%$ to $30 \%$, whereas the length of the drift compression section is estimated correctly to within $5 \%$ for the cases under consideration.

\subsection{Sensitivity Studies}

Now that we have the designs for an IRE drift compression, we can check their sensitivity to various errors that may occur. Specifically, we will be looking at systematic errors in the velocity tilt applied to the beam, as well as at random and systematic errors in the initial beam current. Simulations starting with random errors in the longitudinal velocity suffered from significant slice overtaking problems. This indicates that kinetic effects are important in the dynamics of a beam with random velocity errors, and therefore that a fluid model, on which Hermes is based, is inadequate to treat this problem. For the sensitivity studies, the same numerical parameters were used as for the Hermes calculations to set up a drift compression lattice (table 4.1).

\subsubsection{Systematic Velocity Errors}

First, we consider the effect of the initial head-to-tail tilt being too small by $1 \%$. In this case, the difference in longitudinal velocity between each slice boundary and the center of the beam was reduced by $1 \%$. The RMS error in the final beam current due to this systematic error in the tilt is shown in table 4.3, together with the increase in the final bunch length and the decrease in the current at the beam center for the three final bunch durations under consideration. The effects of the error in the initial tilt are more severe if 
we aim for a shorter final pulse duration. Whereas the final bunch length increased by $4.5 \%$ for a $20 \mathrm{~ns}$ final pulse duration, for the case of a $10 \mathrm{~ns}$ final pulse duration it increased by 10.3\%. Similarly, the current at the beam center at the end of drift compression decreased by $3.6 \%$ for a 20 ns final pulse duration, whereas the current decreased by $6.9 \%$ for the case of a $10 \mathrm{~ns}$ final pulse duration. Figure 4.10 shows the final beam pulse for a $1 \%$ decrease in the initial tilt for the case of a 10 ns final pulse duration.

\begin{tabular}{|c|c|c|c|}
\hline $\begin{array}{c}\text { Final beam } \\
\text { duration (ns) }\end{array}$ & $\begin{array}{c}\text { RMS current } \\
\text { deviation (\%) }\end{array}$ & $\begin{array}{c}\text { Change in final } \\
\text { beam current (\%) }\end{array}$ & $\begin{array}{c}\text { Increase in } \\
\text { bunch length (\%) }\end{array}$ \\
\hline \hline 20 & 3.78 & -3.56 & 4.46 \\
\hline 15 & 5.15 & -4.34 & 5.50 \\
\hline 10 & 8.25 & -6.94 & 10.28 \\
\hline
\end{tabular}

Table 4.3: RMS deviation from the initially specified beam current at the end of drift compression, if the initial head-to-tail velocity tilt is $1 \%$ too low.

\subsubsection{Random Current Errors}

Next, we consider the sensitivity of the IRE drift compression designs to random errors in the initial current. A random error in the current can be expanded in spectral components:

$$
\delta I(z)=\sum_{n=1}^{\infty} A_{n} \cos \left(2 \pi n \frac{z}{\ell}-\phi_{n}\right),
$$

in which $\ell$ is the length of the beam, and $A_{n}$ and $\phi_{n}$ are random numbers. In practical calculations, a limited number of terms $N$ was used. The amplitude $A_{n}$ had a Gaussian distribution with a standard deviation $f I \sqrt{\frac{2}{N}}$, in which $I$ is the beam current and $f$ is the fractional error we want to impose on the beam current in the simulation. The phase angle $\phi_{n}$ is a random variable with a uniform distribution between 0 and $2 \pi$. At any position $z$ along the beam, the standard deviation of the error in the current is then $f I$, independent 


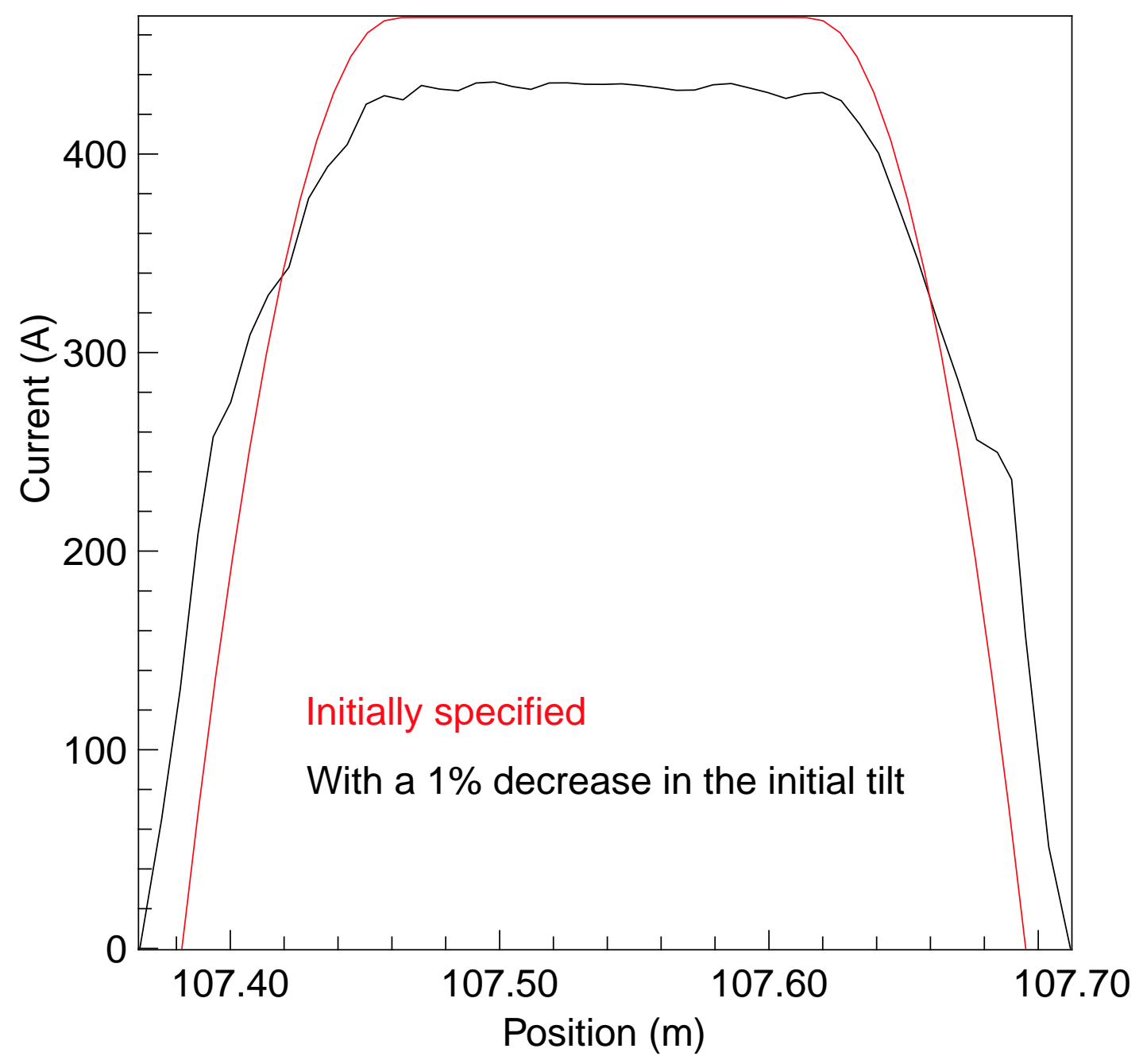

Figure 4.10: The current profile at the end of drift compression, as a function of position, for the case of a $10 \mathrm{~ns}$ final pulse duration if the initial head-to-tail velocity tilt is $1 \%$ too low. 


\begin{tabular}{|c|c|}
\hline $\begin{array}{c}\text { Final beam } \\
\text { duration (ns) }\end{array}$ & $\begin{array}{c}\text { RMS current } \\
\text { deviation (\%) }\end{array}$ \\
\hline \hline 20 & $2.4 \pm 0.9$ \\
\hline 15 & $3.0 \pm 1.9$ \\
\hline 10 & $6.0 \pm 2.5$ \\
\hline
\end{tabular}

Table 4.4: RMS deviation from the initially specified beam current at the end of drift compression, due to $1 \%$ RMS random errors in the initial current.

of the number of terms $N$ that are taken in the sum. In the simulations presented here, $N=10$ terms were used in the sum, with a fractional error $f=1 \%$. Instead of imposing an error on the current (which is a derived quantity in Hermes) directly, the charge in each slice was given a random error based on the prescription given above. As before, the effects of this error on the final beam pulse was measured by calculating the RMS current deviation.

Table 4.4 lists the RMS current deviation that was found for the three drift compression systems that are being considered. Each case was repeated ten times to find the average and the standard deviation of the RMS current deviation at the end of drift compression. Note that the errors listed in table 4.4 include the error due to rematching at the beginning of drift compression. Beams with different final pulse durations are affected similarly by the random error in the initial current. The RMS current deviation at the end of drift compression increased from $1.0 \%$ to $2.4 \%$ for the case with a 20 ns final pulse duration, and from $4.5 \%$ to $6.0 \%$ for the case with a 10 ns final pulse duration. The spread in the RMS current deviation is smallest for the case of a 20 ns final pulse duration (0.9\%), and largest for the case of a 10 ns final pulse duration (2.5\%). Figure 4.11 shows an example of the final beam pulse for a 10 ns final pulse duration beam, in which a $1 \%$ random current variation was applied initially. The current profile shows local deviations from the initially specified final beam pulse, but the overall current profile is not affected. 


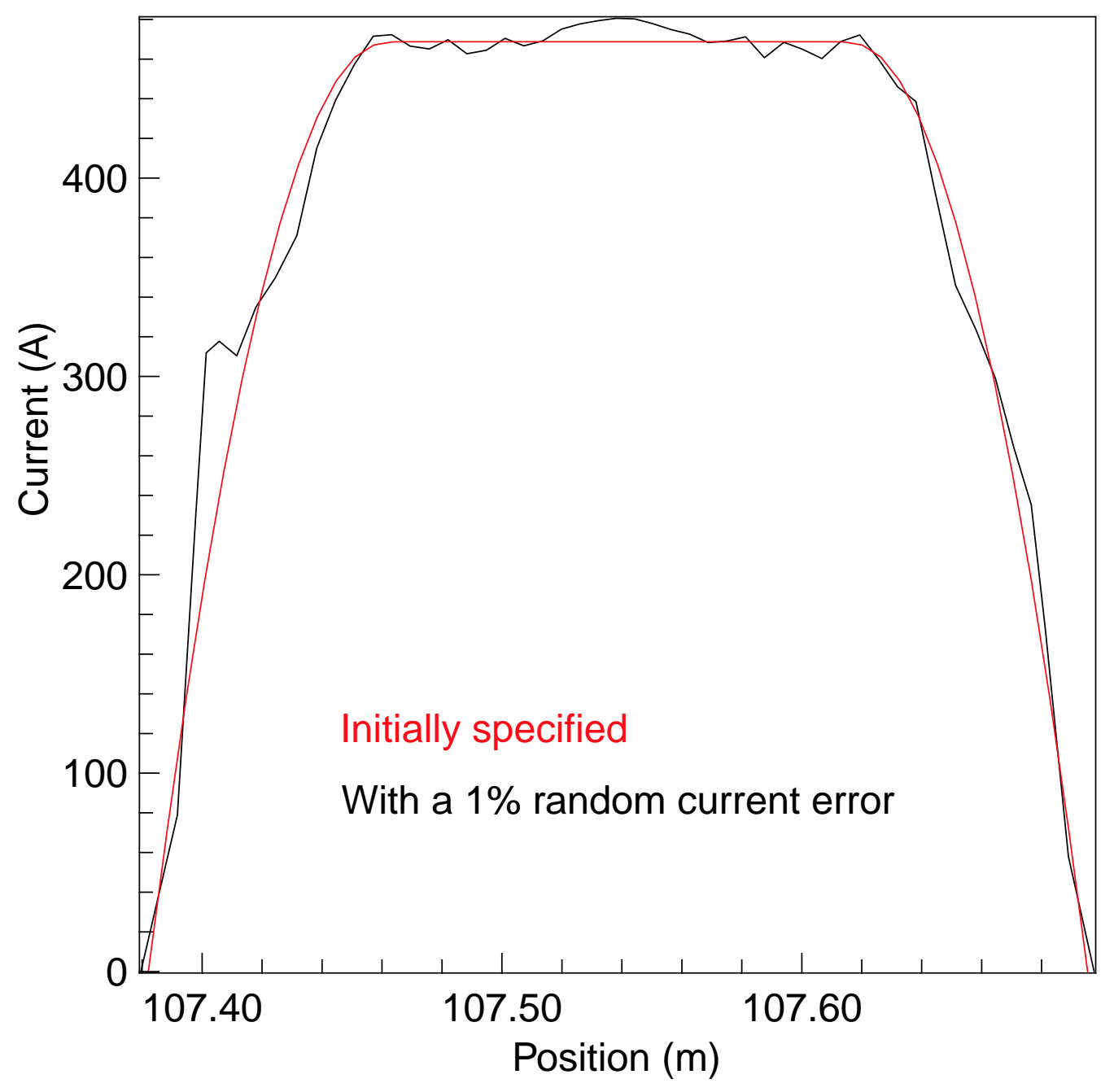

Figure 4.11: An example of the current profile at the end of drift compression, as a function of position, for a $10 \mathrm{~ns}$ final pulse duration, if the initial current has a $1 \%$ random error. 


\subsubsection{Systematic Current Errors}

Finally, we consider the sensitivity of the IRE drift compression designs to a small systematic error in the initial beam current. To study the effects of this error, the charge in all the beam slices was reduced by $1 \%$. In the calculation of the RMS current deviation, the final beam current profile was compared to the initially specified final beam current, multiplied by 0.99 . Table 4.5 lists the RMS current deviation that was found for the three drift compression systems that are being considered. The RMS current deviation does not significantly increase in any of the cases, particularly for shorter final pulse durations. For the case with a 20 ns final pulse duration, the RMS current deviation increased from $0.97 \%$ due to rematching alone, to $1.32 \%$ if we include the systematic current error. For the case with a 10 ns final pulse duration though, the RMS current deviation deviation is $4.50 \%$ with and without the systematic current error. Figure 4.12 shows the final beam pulse for a beam with a 10 ns final pulse duration.

\begin{tabular}{|c|c|}
\hline $\begin{array}{c}\text { Final beam } \\
\text { duration }(\mathrm{ns})\end{array}$ & $\begin{array}{c}\text { RMS current } \\
\text { deviation }(\%)\end{array}$ \\
\hline \hline 20 & 1.32 \\
\hline 15 & 1.36 \\
\hline 10 & 4.50 \\
\hline
\end{tabular}

Table 4.5: RMS deviation from the initially specified beam current multiplied by 0.99, at the end of drift compression, if the initial current is too low by $1 \%$. 


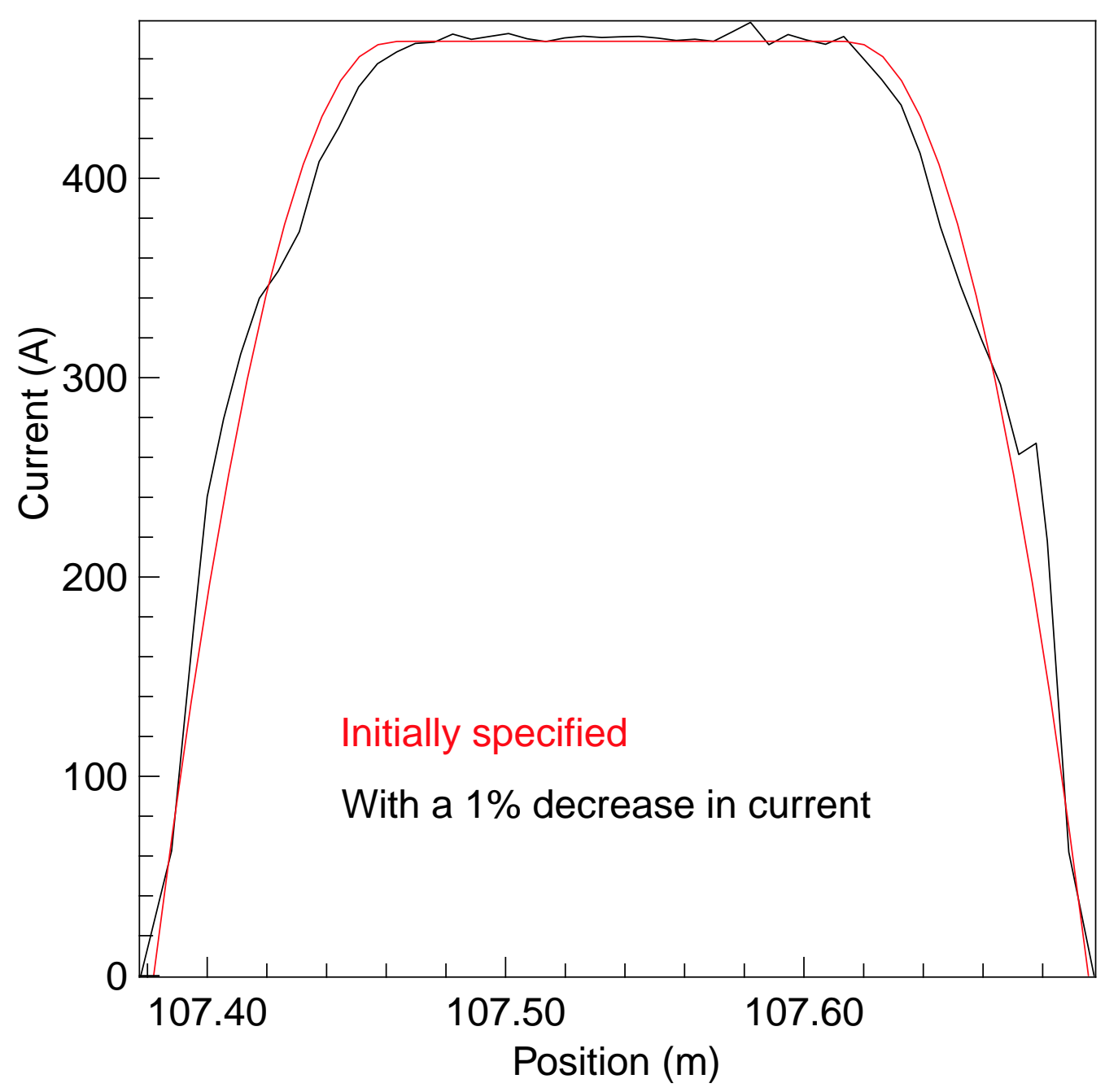

Figure 4.12: The current profile at the end of drift compression, as a function of position, for a $10 \mathrm{~ns}$ final pulse duration, if the initial current is too low by $1 \%$. 


\section{Chapter 5}

\section{The IRE Final Focus System}

In the previous chapter, we described how a beam at the end of the IRE accelerator can be compressed longitudinally in order to increase the energy deposition rate on target. In this chapter, several IRE final focus systems are discussed that focus the beam onto a small spot on the target. We will be particularly interested in how space-charge and third-order aberrations affect the beam quality and the achievable spot size.

Usually, in the final focus system the beam is first expanded transversely before it is focused onto a small spot. The transverse expansion increases the force exerted by the final focus lenses on the beam, which is proportional to the beam radius as shown by the envelope equations $(2.44),(2.45)$. To achieve a small spot size, it is desirable to use a large convergence angle to focus the beam onto a spot.

As the beam expands, the paraxial approximation becomes less valid, and geometric as well as fringe field aberrations may become significant. In addition, space-charge nonlinearities may affect the beam quality, as discussed in section 6.1.1. Third order aber- 
rations become more severe as the convergence angle increases. It has been proposed previously [48] to use octupoles to correct for third-order aberrations in final focus. However, we may be able to reduce the spot size by rematching the final focus lenses, if we take the effects of the third-order aberrations into consideration. In this chapter, an IRE final focus system was used as an example to demonstrate rematching of the final focus system. The effects of third-order aberrations were analyzed for different values of the convergence angle, as well as the effectiveness of rematching the final focus system to reduce the spot size.

\subsection{Design of a Final Focus System}

Each beam in the preliminary IRE design has a charge of $4.6875 \mu \mathrm{C}$, at an energy of $200 \mathrm{MeV}$, as shown in table 1.2. For the final focus designs discussed here, we will assume that the beam is compressed to a duration of $5 \mathrm{~ns}$ with a flat beam profile. This corresponds

to a perveance of $1.33 \times 10^{-3}$ in the final focus system. To focus such high-perveance beams, partial or complete neutralization of the beam charge is required following the final lens. For the final focus designs presented here, the beam was assumed to be neutralized to $98 \%$ of its initial perveance $10 \mathrm{~cm}$ after the last magnet. In the simulation, this was done by reducing the charge of each macroparticle by $98 \%$.

The standoff distance between the end of the last magnet and the focal spot was chosen to be $2 \mathrm{~m}$. For the simulations presented here, the convergence angle was calculated at the neutralization point. For a given final spot radius, we can integrate the envelope equation (2.44), (2.45) backward from the final focus spot to the neutralization point, assuming a round beam, to find the convergence angle. 
Given the perveance of the beam before neutralization $Q=1.33 \times 10^{-3}$, and choosing the average beam radius in the final focus system to be $\bar{a}=6 \mathrm{~cm}$, we can find the lattice half period $L$ from equation (2.60). For the phase advance per period, $\sigma_{0}=53.77^{\circ}$ was used, which corresponds to its value in the IRE accelerator. This yields a half-lattice period of $0.902 \mathrm{~m}$. Once we know the half lattice period and using a value of $65 \%$ for the occupancy $\eta$, which is near its practical achievable limit, we can set up a lattice system consisting of four quadrupoles. The initial beam parameters $a, b, a^{\prime}, b^{\prime}$ were chosen such that they correspond to the matched conditions for a beam propagating in the lattice with the given phase advance $\sigma_{0}$ and an average beam radius of $6 \mathrm{~cm}$. This means that the beam at the beginning of the final focus system resembles a beam coming out of a transport section or the drift compression section.

Using the initial beam parameters, we can find the quadrupole strengths that match the beam to the final beam parameters at the neutralization point that are needed to achieve a given spot size. A Python script, based on JavaEnvelope, was written for this purpose. We then have a final focus system to converge a beam coming out of a transport section onto a spot with a given radius. Figure 5.1 shows the focusing solutions that were found for convergence angles between 10 to $20 \mathrm{mrad}$, whereas the corresponding magnetic field gradients of the four quadrupoles are shown in figure 5.3.

\section{$5.2 \quad$ Third-Order Aberrations}

The final focus system described above focuses a beam onto a spot with a given radius in the absence of nonlinearities, such that the envelope equations (2.44), (2.45) are 

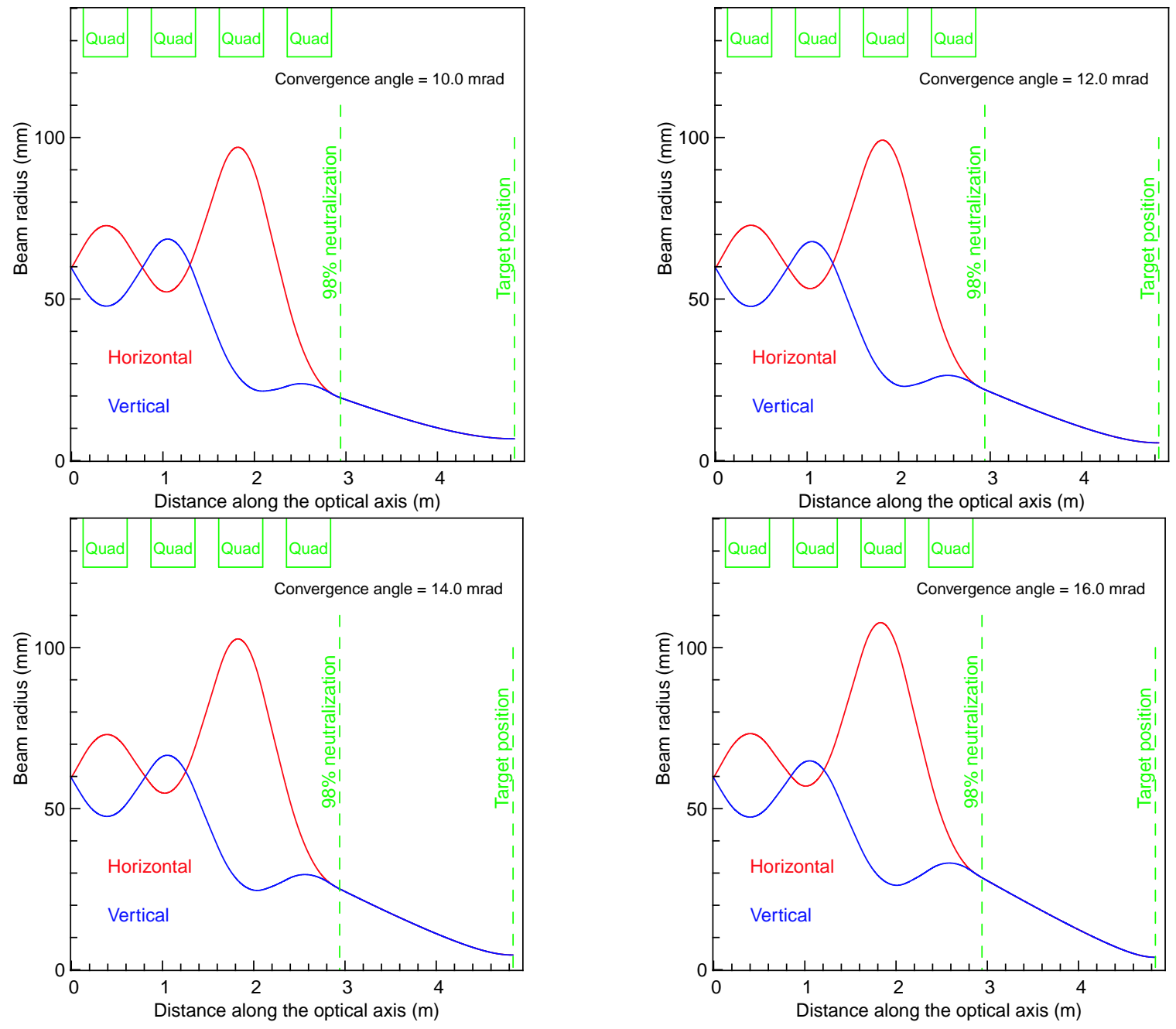

Figure 5.1: The focusing solutions for different values of the convergence angle, as calculated from the envelope equations. Continued on next page. 

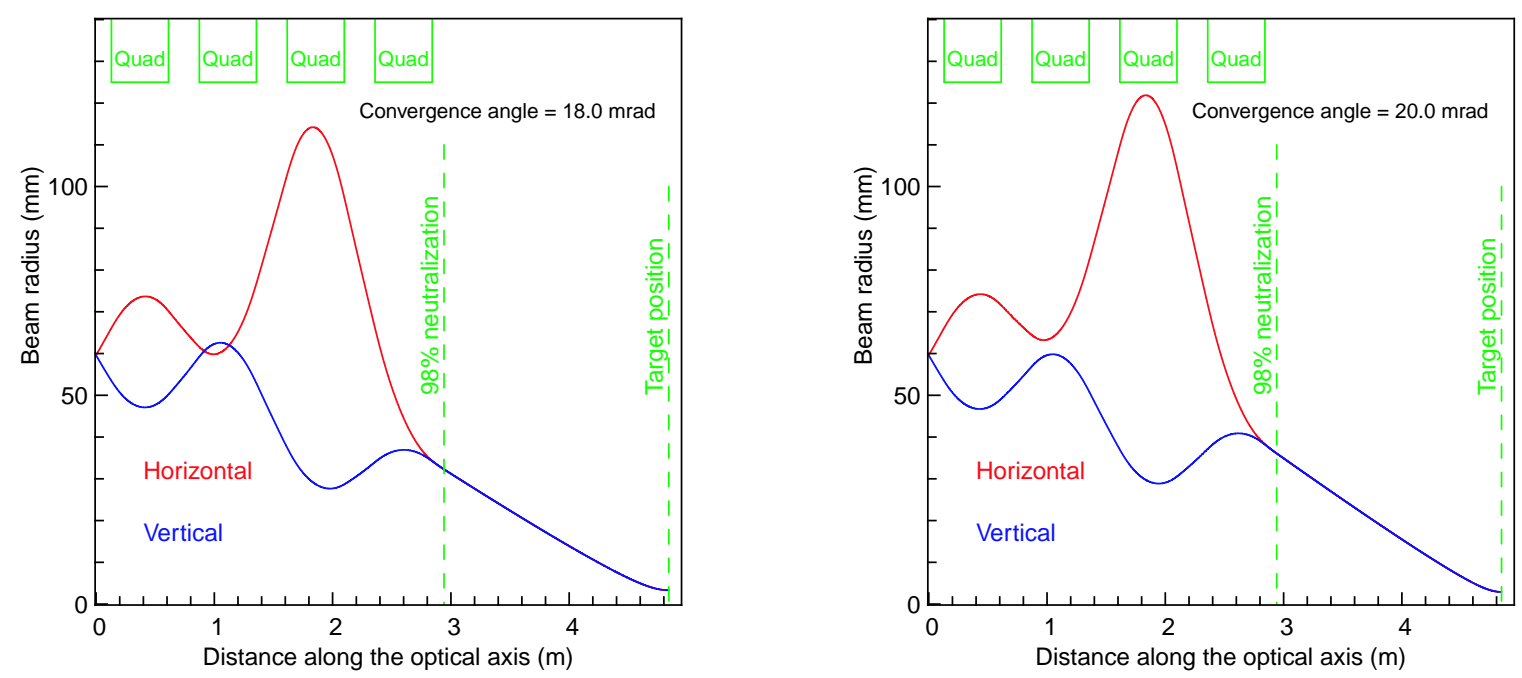

Figure 5.2: Continuation of figure 5.1.

valid. In this approximation, for a given standoff distance of $2 \mathrm{~m}$, the final spot size decreases if the convergence angle is increased. The final spot size as a function of the convergence angle, as calculated from the envelope equations, is shown in figure 5.4.

Nonlinear space-charge fields or aberrations may cause an increase in the final spot size. As the convergence angle increases, the effects of third-order aberrations become more severe. Therefore, for large convergence angles we expect the final spot radius to increase. An optimal convergence angle will then exist for which the final focus spot size, in the presence of nonlinear space charge and third-order aberrations, is minimized.

The transverse dynamics of a beam was simulated with the WARPxy code, including nonlinear space-charge and third-order aberrations, for different values of the convergence angle. In these simulations, we assumed the quadrupoles to have fringe fields of the shape given by equation (2.73), with a fringe length $L_{\mathrm{ff}}=15 \mathrm{~cm}$. Table 5.1 lists the simulation parameters. The beam was surrounded by a circular conducting pipe, whose 

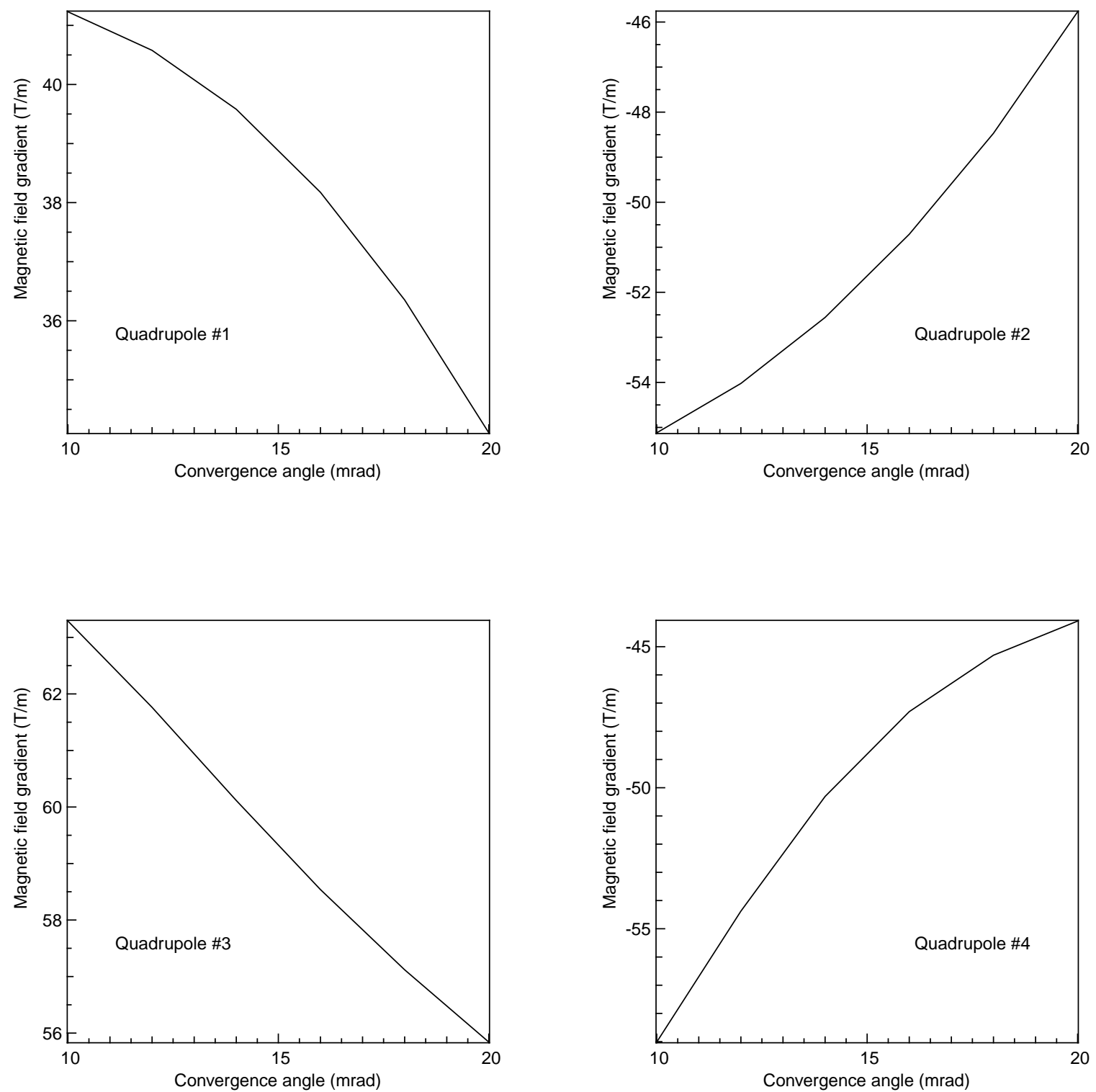

Figure 5.3: The magnetic field gradients of the four quadrupoles as a function of the convergence angle, as calculated from the envelope equations. 


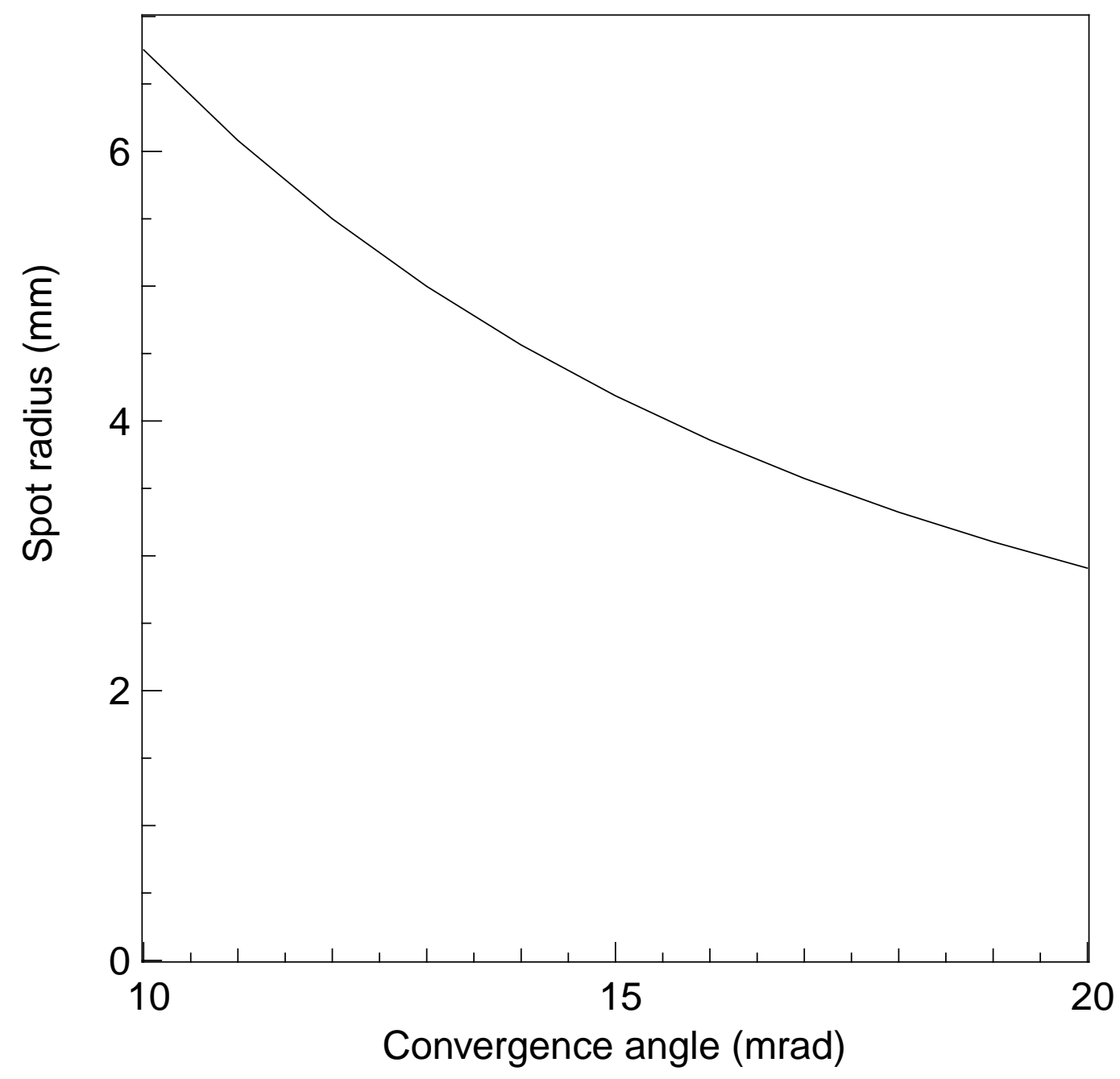

Figure 5.4: The focal spot radius as a function of the convergence angle, as calculated from the envelope equations. 


\begin{tabular}{|l|l|}
\hline Initial distribution & $\mathrm{K}-\mathrm{V}$ or semi-Gaussian \\
\hline Number of particles & 40,000 (none lost in any of the simulations) \\
\hline Grid size & $128 \times 128$ \\
\hline Step size & $\begin{array}{l}1 \mathrm{~mm} \text { inside quadrupoles and their fringes } \\
1 \mathrm{~cm} \text { elsewhere }\end{array}$ \\
\hline
\end{tabular}

Table 5.1: Simulation parameters for the IRE final focus simulations.

radius was chosen according to the heuristic [28]

$$
\text { pipe radius }=1.25 \times \text { maximum beam radius }+5 \mathrm{~mm} \text {, }
$$

The grid size was then chosen such that this pipe just fits inside the grid. By choosing a circular conducting boundary at a sizeable distance from the beam, we can avoid any image charges significantly affecting the beam.

Figure 5.5 shows the focal spot radius as a function of the convergence angle that was found from the WARPxy simulations, both for an initially semi-Gaussian beam and for a $\mathrm{K}-\mathrm{V}$ beam. The spot radius was calculated from $r_{\mathrm{spot}}=\sqrt{a b}$. It is significantly larger than what is predicted by the envelope equation, particularly for large convergence angles. The minimum spot radius is achieved for a convergence angle of $16 \mathrm{mrad}$. The spot radius increases for larger convergence angles due to the third-order aberrations. Since the difference between the initially semi-Gaussian beam and the $\mathrm{K}-\mathrm{V}$ beam is small, we conclude that space-charge nonlinearities are not important for the IRE final focus designs presented here.

Figure 5.6 shows the horizontal and vertical beam radius that was found from the WARPxy simulations. Due to the third-order aberrations, the beam is no longer circular at the target. This suggests that if third-order aberrations are included, the quadrupoles 


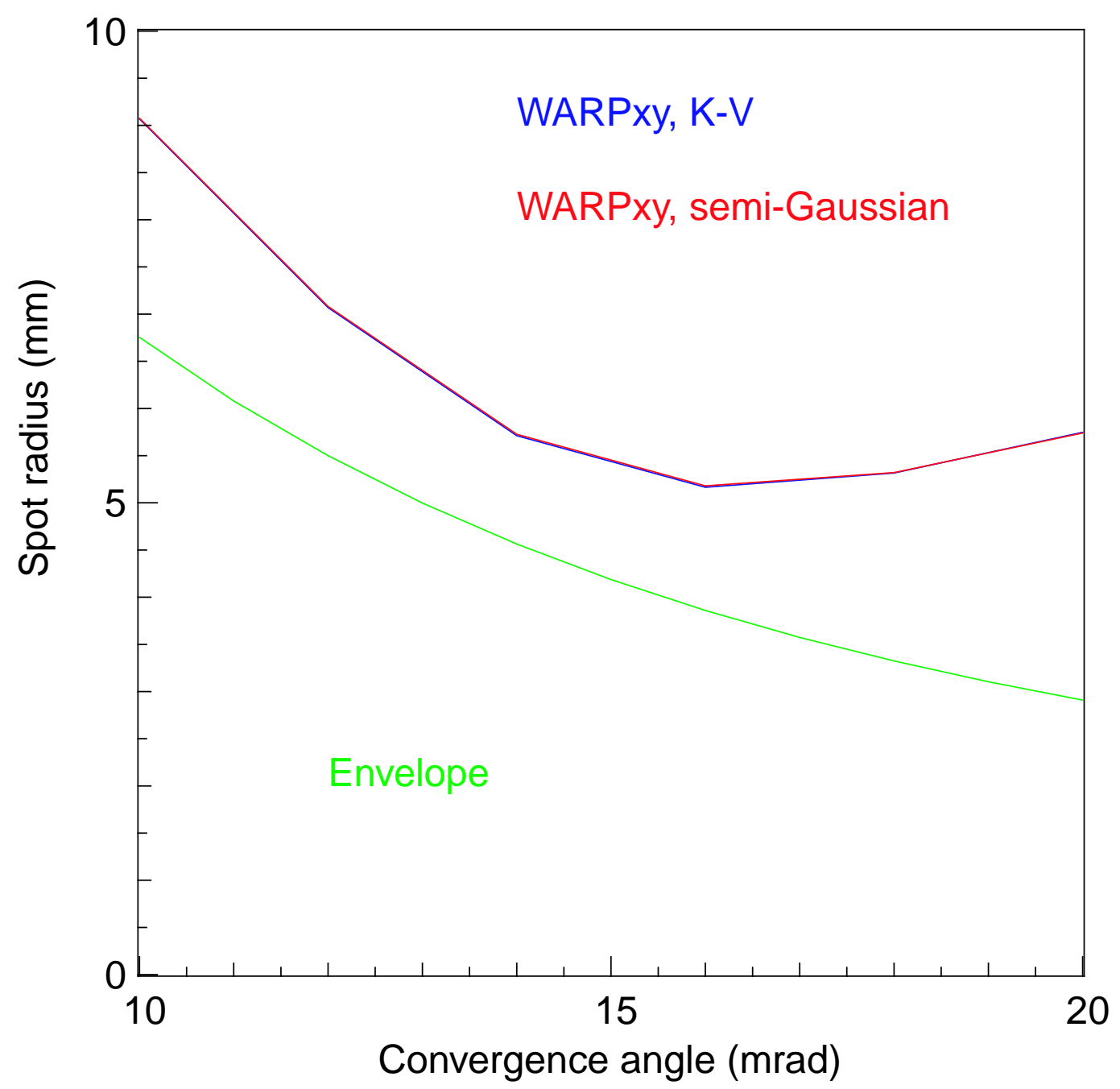

Figure 5.5: The focal spot radius as a function of the convergence angle, as calculated from the envelope equations, and from WARPxy simulations, taking into account third-order and space-charge aberrations. Note that the final spot radius is virtually independent of the initial distribution being $\mathrm{K}-\mathrm{V}$ or semi-Gaussian. 
are no longer optimally tuned. We expect therefore to be able to improve on the final spot size by readjusting the quadrupoles.

\subsection{PIC Code Rematching}

In this section, we will discuss how to find the optimal quadrupole strengths, including the nonlinear effects that are captured by a PIC simulation. We are looking for a set of quadrupole field strengths that focus the beam unto a round spot at the target position, with $a^{\prime}=b^{\prime}=0$, for a given convergence angle $\theta_{0}$ at the neutralization point. Effectively, this problem reduces to finding the root of the set of four equations

$$
\underline{y}=\underline{f}(\underline{x}),
$$

in which $\underline{y}=\left(\theta-\theta_{0}, a-b, a^{\prime}, b^{\prime}\right), \underline{x}$ is a vector that holds the magnetic field gradients of the four quadrupoles, and $\underline{f}$ represents a WARPxy simulation. In this equation, $a, b, a^{\prime}$, $b^{\prime}$ are evaluated from the simulation data at the target position, and $\theta \equiv \frac{1}{2}\left(a^{\prime}+b^{\prime}\right)$ is the convergence angle at the neutralization point that was found from the simulation.

The algorithm to solve this equation is based on the Newton-Raphson method [80]. Whereas in one dimension the derivative of the function $f$ is used to find its zero, in more than one dimension we make use of the Jacobian matrix $J_{i j}=\partial f_{i} / \partial x_{j}$. We can then expand $\underline{f}$ in a Taylor series:

$$
\underline{f}(\underline{x}+\delta \underline{x})=\underline{f}(\underline{x})+\underline{\underline{J}} \cdot \delta \underline{x}+O(\delta \underline{x})^{2} .
$$

We can solve $\underline{f}(\underline{x}+\delta \underline{x})=0$ by neglecting the $O(\delta \underline{x})^{2}$ term:

$$
-\underline{f}(\underline{x})=\underline{J} \cdot \delta \underline{x}
$$



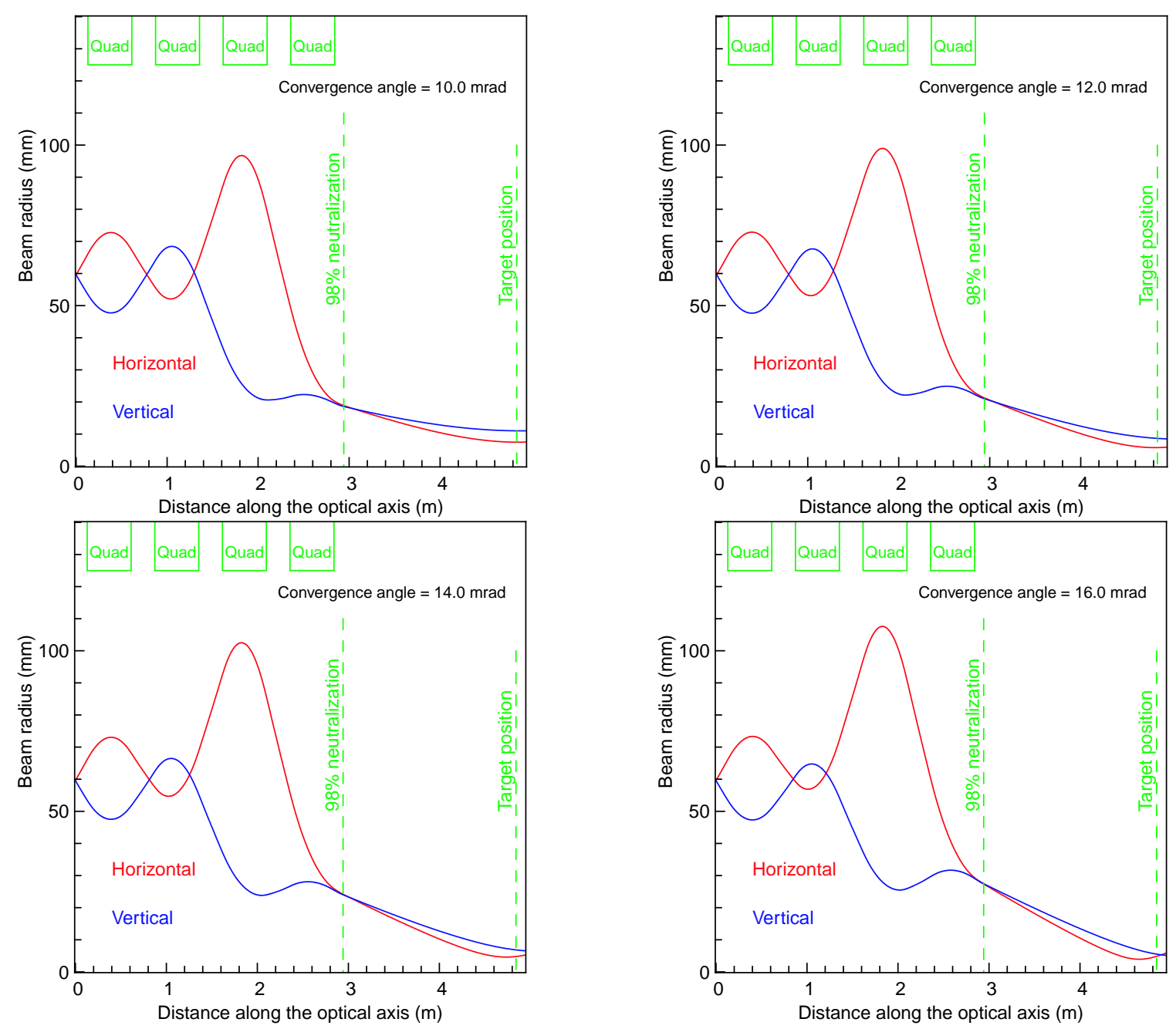

Figure 5.6: The horizontal and vertical beam radius as calculated from the WARPxy simulations, for different values of the convergence angle, using the magnetic field gradients that were found from the envelope equations. For clarity, the quadrupoles are drawn closer to the beam than they were in the simulations. Continued on next page. 

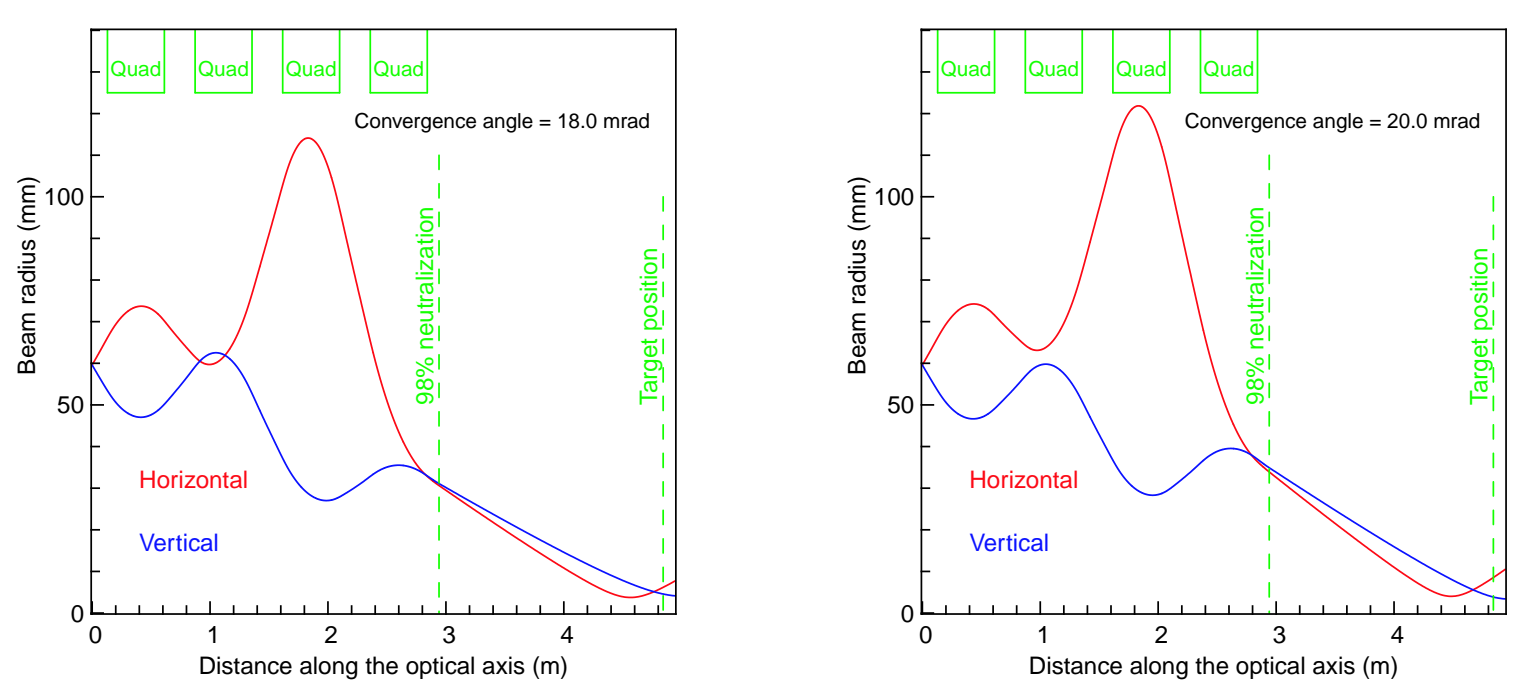

Figure 5.7: Continuation of figure 5.6.

and solving this linear set of equations for $\delta \underline{x}$. Based on an original estimate $\underline{x}_{\text {orig }}$ of the zero of $\underline{f}(\underline{x})$, we now have a new estimate $\underline{x}_{\text {new }}=\underline{x}_{\text {orig }}+\delta \underline{x}$ in which $\delta \underline{x}$ is given by

$$
\delta \underline{x}=-\underline{\underline{J}}^{-1} \cdot \underline{f}(\underline{x})
$$

By iterating this equation, and reevaluating the Jacobian matrix $\underline{\underline{J}}$ on each iteration step, we can converge to a zero of $\underline{f}(\underline{x})$. Convergence is not guaranteed though. It depends on the existence of a zero of $\underline{f}(\underline{x})$, as well as the starting point of the iteration $\underline{x}_{\text {orig }}$.

If the initial guess $\underline{x}_{\text {orig }}$ is in a region where the function $\underline{f}(\underline{x})$ varies rapidly, a step $\delta \underline{x}$ based on a first-order Taylor expansion may produce an $\underline{x}_{\text {new }}$ whose error, as measured by the difference between $\underline{f}\left(\underline{x}_{\text {new }}\right)$ and zero, may actually be larger than the original error. In those cases, the iteration step is repeated in the same direction as the first step, but with a smaller step size. The new step size is found by fitting a parabola to the function $\underline{f}(\underline{x})$ along the direction of the step, using the function value and the derivative at $\underline{x}_{\text {orig }}$ and the function value at $\underline{x}_{\text {new }}$. This avoids taking steps that increase the error, as well as 
evaluating the Jacobian matrix anew.

Appendix A.2 contains the Python script in which this algorithm was implemented. First one WARPxy simulation was done using the magnetic field gradients that were found from the envelope equations. This gives the initial error $\underline{f}\left(\underline{x}_{\text {orig }}\right)$. The Jacobian matrix was calculated by varying the magnetic field gradient of each of the magnets separately, and finding the effect on $\underline{y}=\left(\theta-\theta_{0}, a-b, a^{\prime}, b^{\prime}\right)$ by running a WARPxy simulation. Four WARPxy simulations are needed to find the Jacobian matrix. Equation (5.5) was then used to find the next set of magnetic field gradients. This procedure converges rapidly for small values of the convergence angle $(\theta \lesssim 14 \mathrm{mrad})$, whereas it converges very slowly for larger convergence angles. To save time, many of the iteration sequences were done for a small number of particles and a large step size initially, starting from many different initial conditions, and gradually improving the accuracy of the simulation when $\underline{x}$ approaches the solution. The final simulation parameters correspond to those given in table 5.1.

Figure 5.8 shows the beam radius at the target position as a function of the convergence angle, after rematching the quadrupoles using WARPxy. Figure 5.9 shows the corresponding magnetic field gradients. For convergence angles less than 16 mrad, a significant improvement in spot radius was achieved by rematching. For larger convergence angles, the requirement of having an equal horizontal and vertical beam radius at the target position in the rematching algorithm actually produces a larger spot size than without rematching. For a convergence angle of $17 \mathrm{mrad}$, halo formation causes a significant number of particles to be lost, if we require the horizontal and vertical beam radius to be equal. The solution for 17 mrad shown in figure 5.8 was achieved by increasing the aperture by $22 \%$ 
to avoid particle loss. Note that no particles were lost for any of the convergence angles in the simulations without rematching of the final focus system.

To understand why particles are being lost, we can look at the focusing solutions that were found by rematching using WARPxy. Figure 5.10 shows that focusing solutions that were found for convergence angles between 10 and 16 mrad. Whereas for low convergence angles the focusing solution is close to what was found using the envelope code (see figure 5.1), at 16 mrad the horizontal and vertical beam radii deviate significantly from what was found using the envelope equations. A much larger excursion is needed for the horizontal beam radius, which means that aberrations affect the beam more strongly. Figure 5.11 shows the focusing solution for a $17 \mathrm{mrad}$ convergence angle. In this case, the excursion in the horizontal beam radius became so large as to make a larger aperture necessary.

Figures 5.12 and 5.13 show the positions of the beam particles in configuration space at the target position for the different convergence angles after rematching. For a convergence angle of $16 \mathrm{mrad}$ or larger, the focal spot is no longer uniform. We can conclude that while rematching provides a smaller spot size at low convergence angles, at larger convergence angles rematching does not significantly improve the final spot characteristics due to halo formation. 


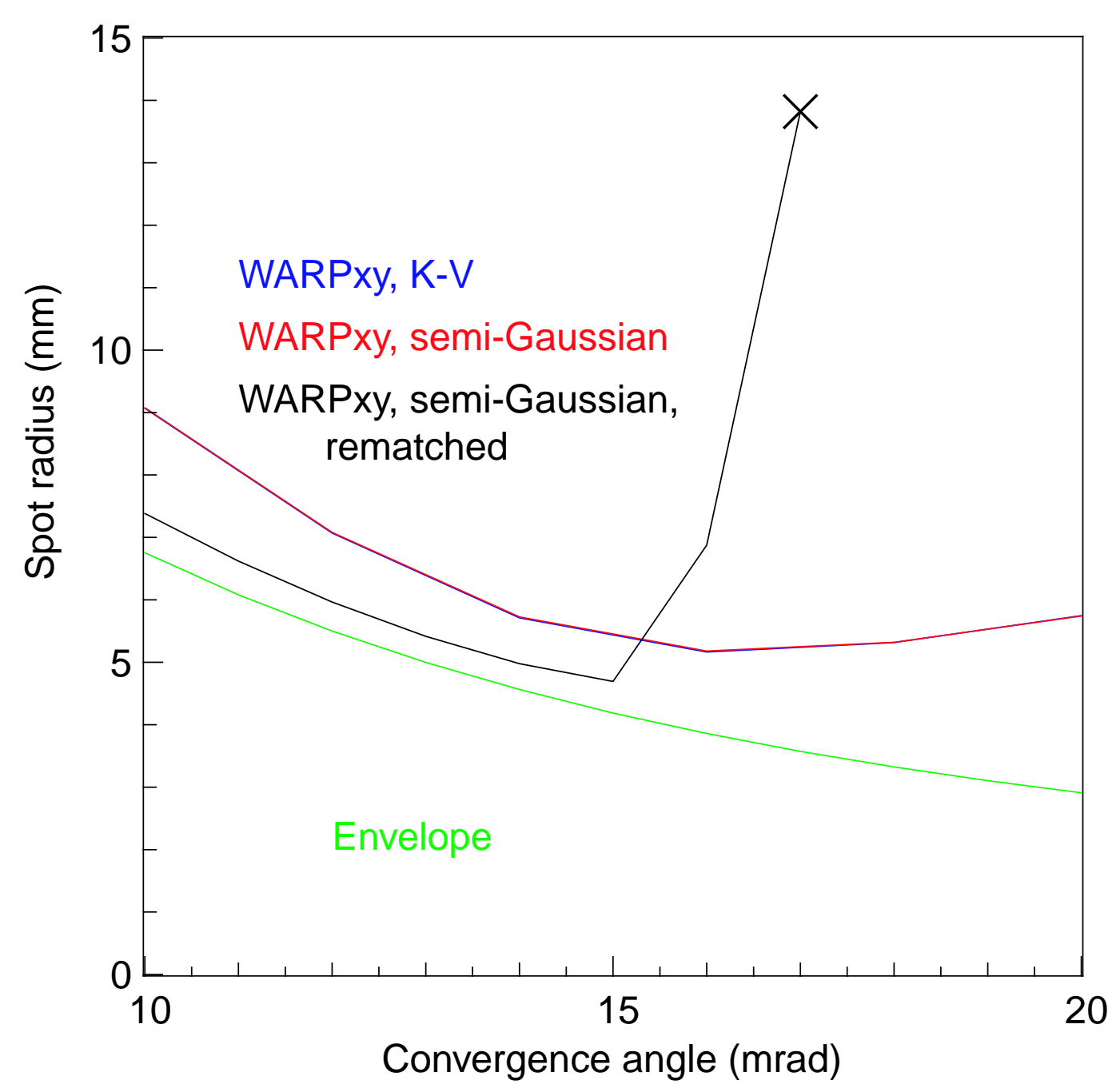

Figure 5.8: The focal spot radius as a function of the convergence angle, as calculated from the envelope equations, and from WARPxy simulations, after rematching the magnetic field gradients of the quadrupoles using WARPxy. For the rematched solution at a $17 \mathrm{mrad}$ convergence angle, the aperture was increased by $22 \%$ to avoid particle loss. Note that the final spot radius is virtually independent of the initial distribution being $\mathrm{K}-\mathrm{V}$ or semiGaussian. 

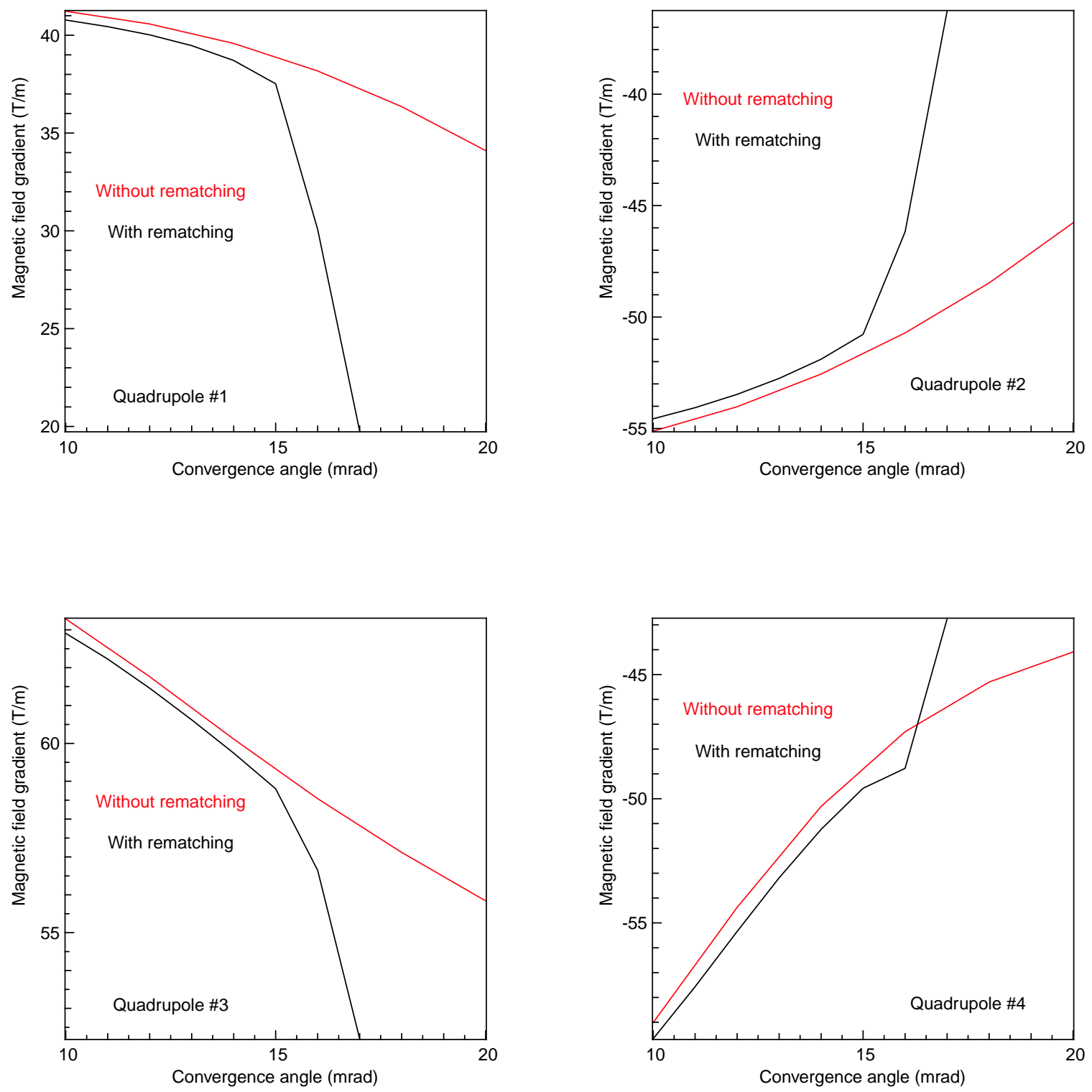

Figure 5.9: The magnetic field gradients of the four quadrupoles as a function of the convergence angle, after rematching using WARPxy. 

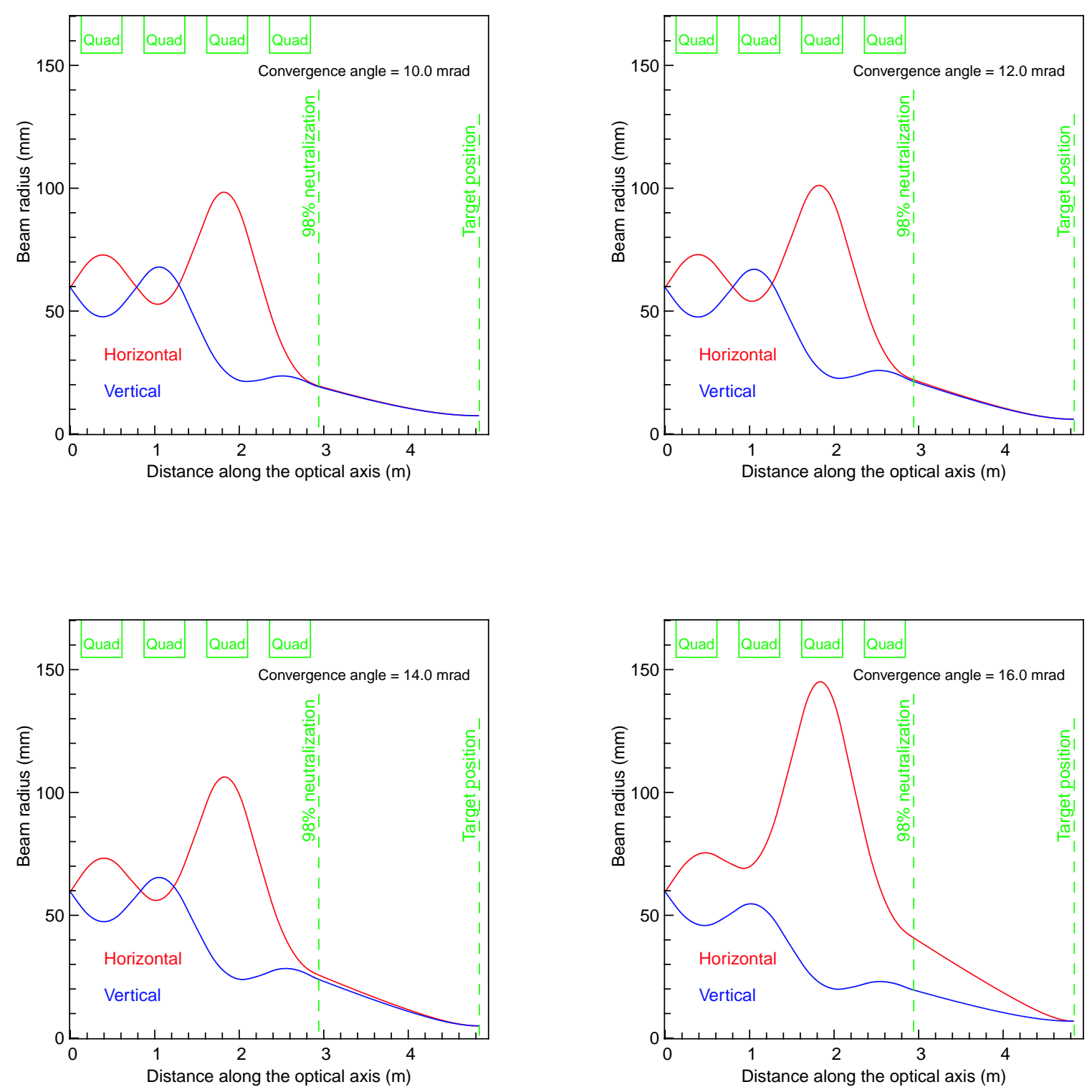

Figure 5.10: The horizontal and vertical beam radius as calculated from the WARPxy simulations, for convergence angles between 10 and $16 \mathrm{mrad}$, after rematching the magnetic field gradients of the quadrupoles using the WARPxy code. 


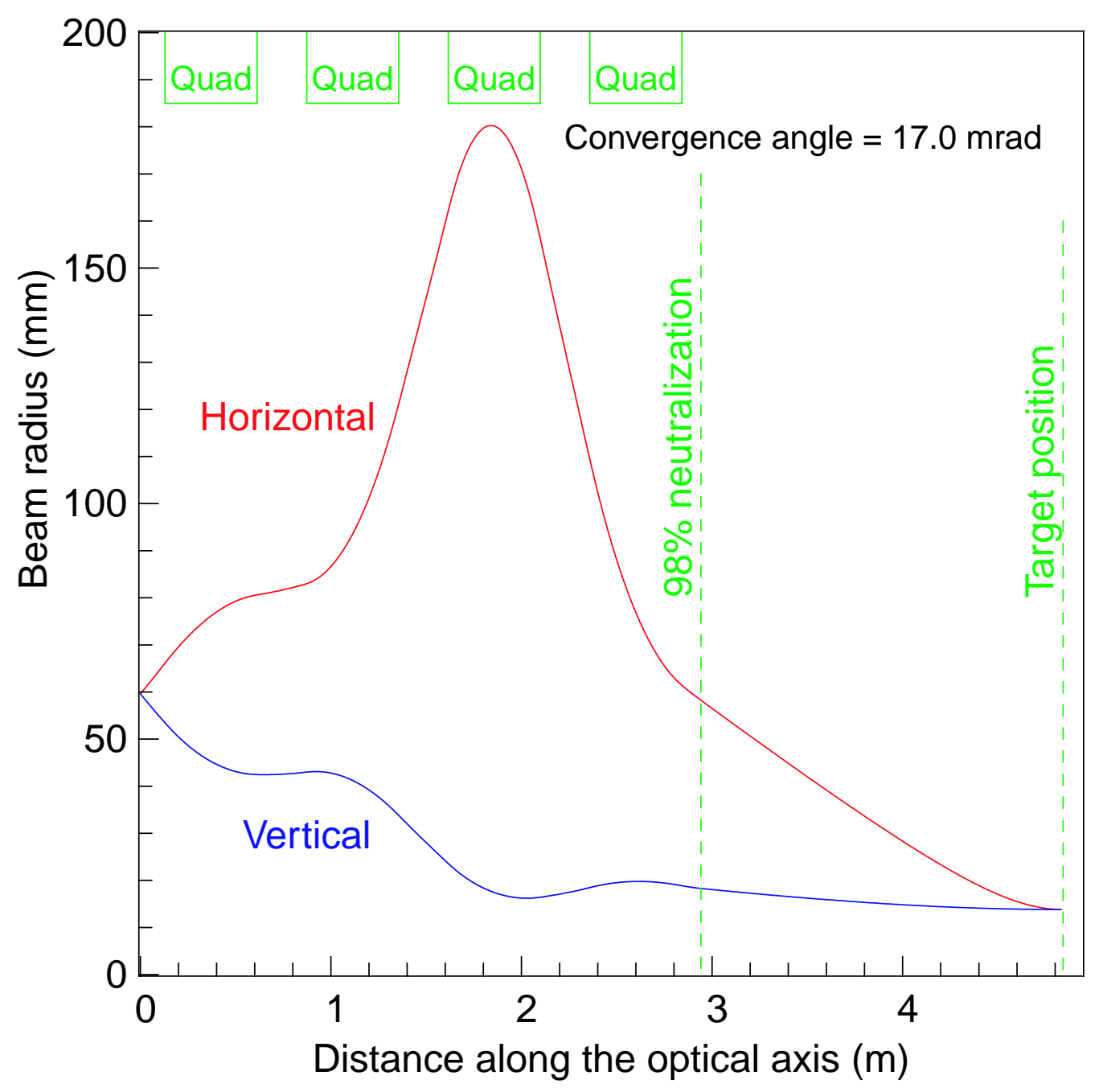

Figure 5.11: The horizontal and vertical beam radius as calculated from the WARPxy simulations, for a $17 \mathrm{mrad}$ convergence angle, after rematching the magnetic field gradients of the quadrupoles using the WARPxy code. 

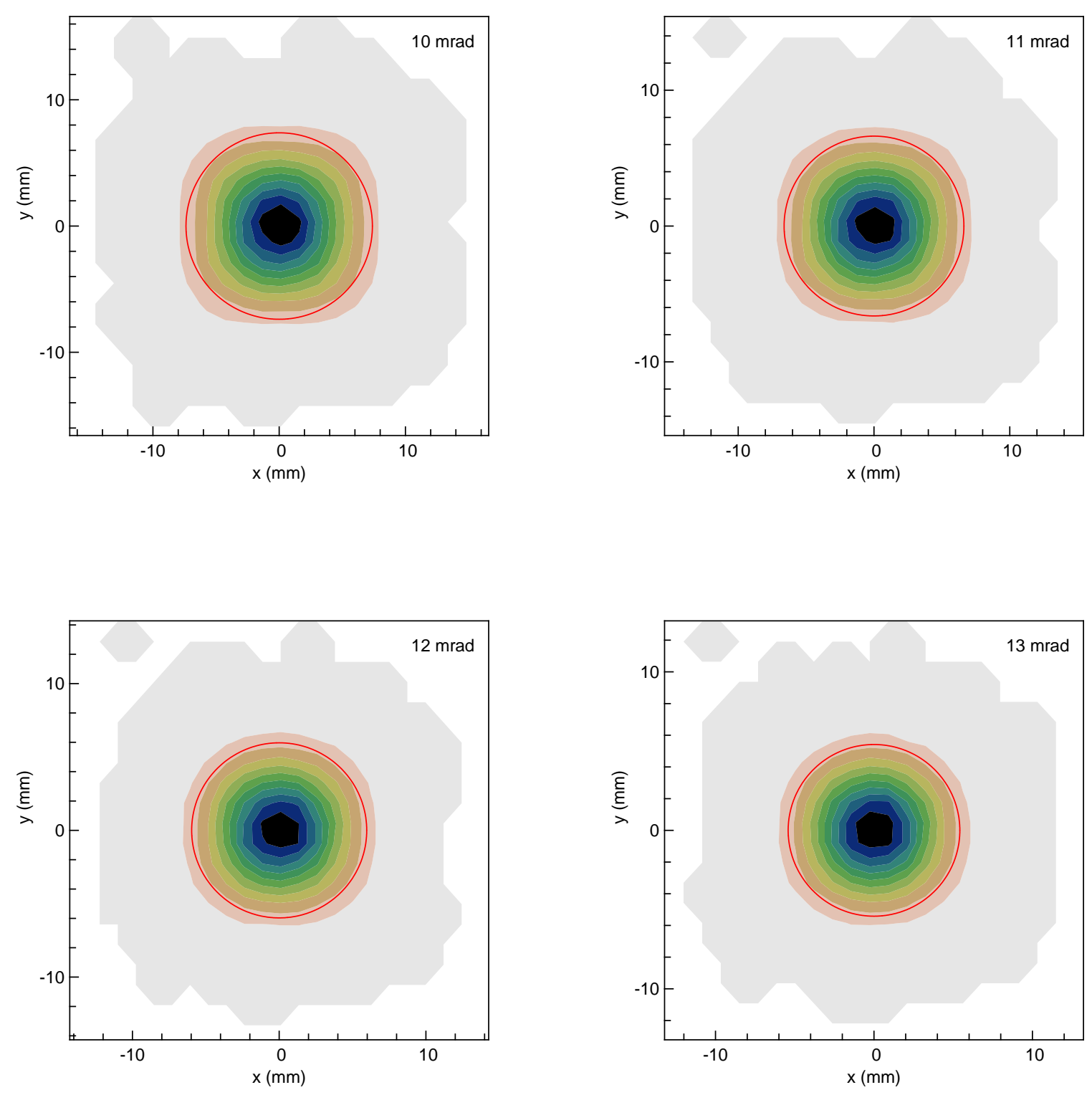

Figure 5.12: The particle density in configuration space at the target location, for different values of the convergence angle, after rematching the magnetic field gradients of the quadrupoles using the WARPxy code. The color-coding of the particle density uses a linear scale. The red circle has a radius equal to $2 \sqrt{\left\langle x^{2}\right\rangle}=2 \sqrt{\left\langle y^{2}\right\rangle}$. Continued on next page. 

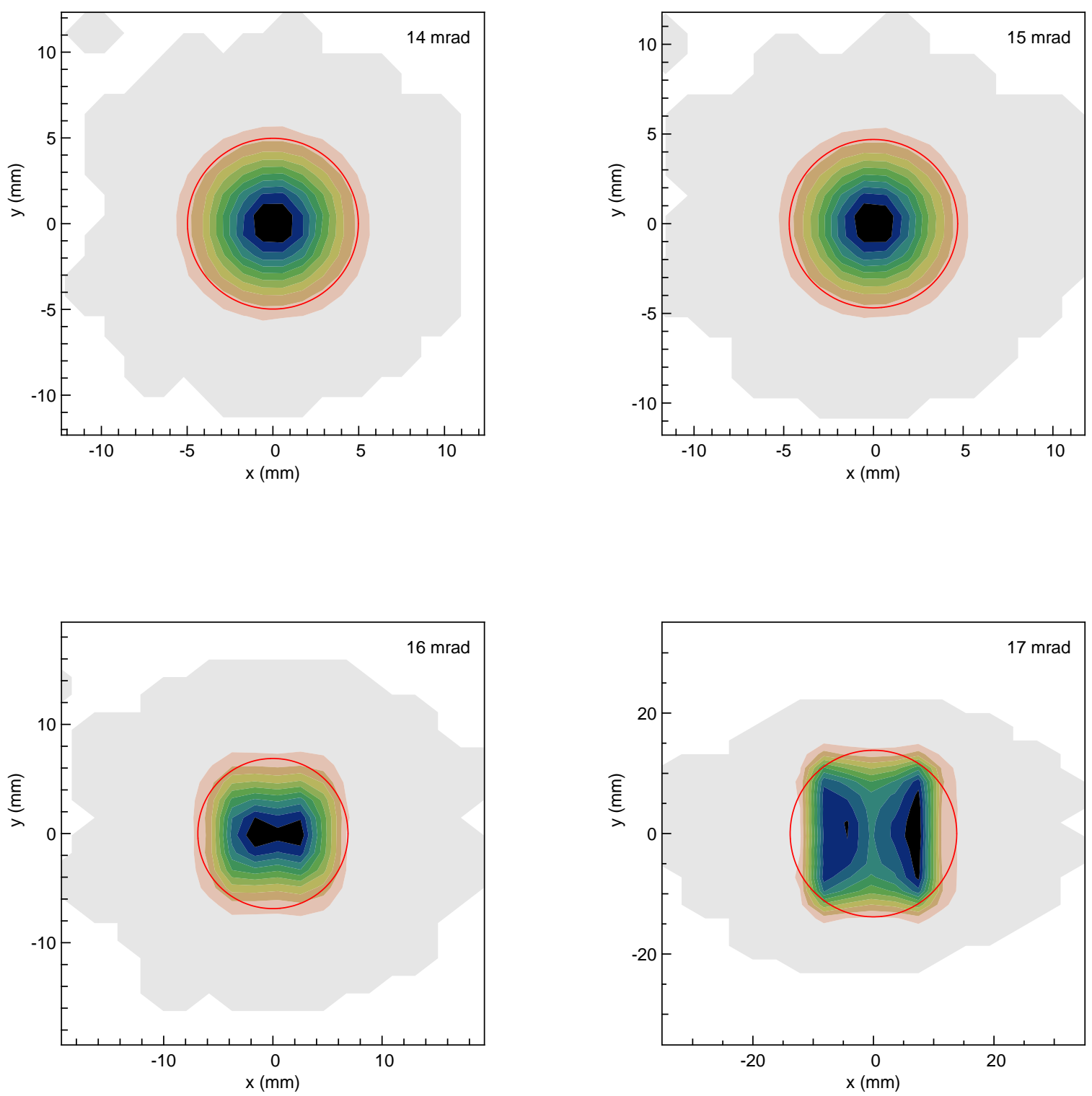

Figure 5.13: Continuation of figure 5.12. 


\section{Chapter 6}

\section{The Scaled Final Focus Experiment}

In the Scaled Final Focus Experiment, the beam parameters and the focusing lattice parameters were scaled such that the relative importance of the various terms in the envelope equations (2.44), (2.45) is maintained. By scaling the HIBALL-II parameters such that all of the physical dimensions length are reduced by a factor of 10 while the perveance is fixed (as described in section 1.4.3), we find a corresponding beam current of $96.5 \mu \mathrm{A}$, an edge emittance of $3 \pi \mathrm{mm}$ mrad, and an ion energy of $160 \mathrm{keV}$ for the scaled experiment [72]. These beam parameters are achieved in the experiment by aperturing the beam coming from the source. Since the size of the aperture determines both the beam current and the emittance, the scaled HIBALL-II beam parameters can only be achieved in approximation. The scaled HIBALL-II case as well as the modified scaled HIBALL-II case discussed below are close to the scaled HIBALL-II current, whereas an additional case with a larger initial current was done in order to perform beam neutralization experiments [69], [72].

Since detailed measurements have been performed on the Scaled Final Focus Ex- 
periment [72], simulations of this experiment is an opportunity for model verification. By comparing experimental and simulation results, the degree to which WARP replicated the experimental reality can be assessed. Special attention was given to nonideal effects present in the experiment, such as fringe fields and image fields. Our knowledge of these effects is often limited. By including some nonideal effects in the simulations though, it can be shown which of them are likely to significantly affect the beam, and which are not.

In the experiment, the beam was often significantly rotated in configuration space after the magnetic section, whereas the beam had been upright after the electrostatic section. Several possible causes of this beam rotation have been proposed, such as quadrupole rotations, the Earth's magnetic field, and asymmetries in the leads to the focusing magnets. Comparisons between simulation and experiment are difficult as long as the true cause of the beam rotation is not identified, and either solved in the experiment or included in the simulation. A series of beam rotation experiments and simulations were performed to address this issue.

The simulations were performed using the WARPxy transverse slice particle-incell code, taking the magnitude of the quadrupole strengths as well as the initial beam parameters from the experiment. In many cases, possibly due to the beam rotation described above, or due to uncertainties in the measured beam current or initial emittance, the envelope found in the simulation did not exactly agree with the experimentally measured envelope. In those cases, the quadrupole strengths were rematched in the simulation in order to resemble the experimentally measured beam envelope more closely.

To accurately replicate the effect of image charges, particular attention was given to 
the presence of conducting boundaries in the experiment. Since WARP uses a sine-transform instead of the regular Fourier transform to solve Poisson's equation, a beam is by default effectively surrounded by a conducting rectangular box of the size of the grid. Due to the lack of symmetry of such a rectangular box, its image charges may significantly affect the beam dynamics if it is not shielded by other conducting boundaries. The electrostatic quadrupoles in the Scaled Final Focus Experiment consist of four half-rods, and were modeled as such in the simulation (figure 6.1). Whereas the axial ends of the half-rods were rounded in the experiment [94], they were modeled as hard edges in the simulations. The magnetic quadrupoles have a metallic liner on their inside, which is represented as a conducting circular boundary in the simulation. In the drift spaces between the quadrupoles, the only conducting surfaces near the beam in the experiment are the diagnostic paddles at a distance of about $10 \mathrm{~cm}$, followed by the vacuum tank in which the beam travels. Since these conducting boundaries are so far removed from the beam, any image charges on them have a negligible effect on the beam. In the simulations, the transverse extent of the conducting boundaries in the drift spaces are therefore represented by a large circular boundary that just fits on the grid.

In some of the simulations presented here, the lowest order nonlinearities due to fringe fields were included. In those simulations, a $1 \mathrm{~mm}$ step size was used inside the quadrupoles and their fringes, compared to a $1 \mathrm{~cm}$ step size outside these regions.

Other nonlinear fields may arise from the current leads into the magnets, and higher order multipoles that may exist in superposition to the quadrupolar field of the focusing elements. These effects have not been taken into consideration in the simulations 


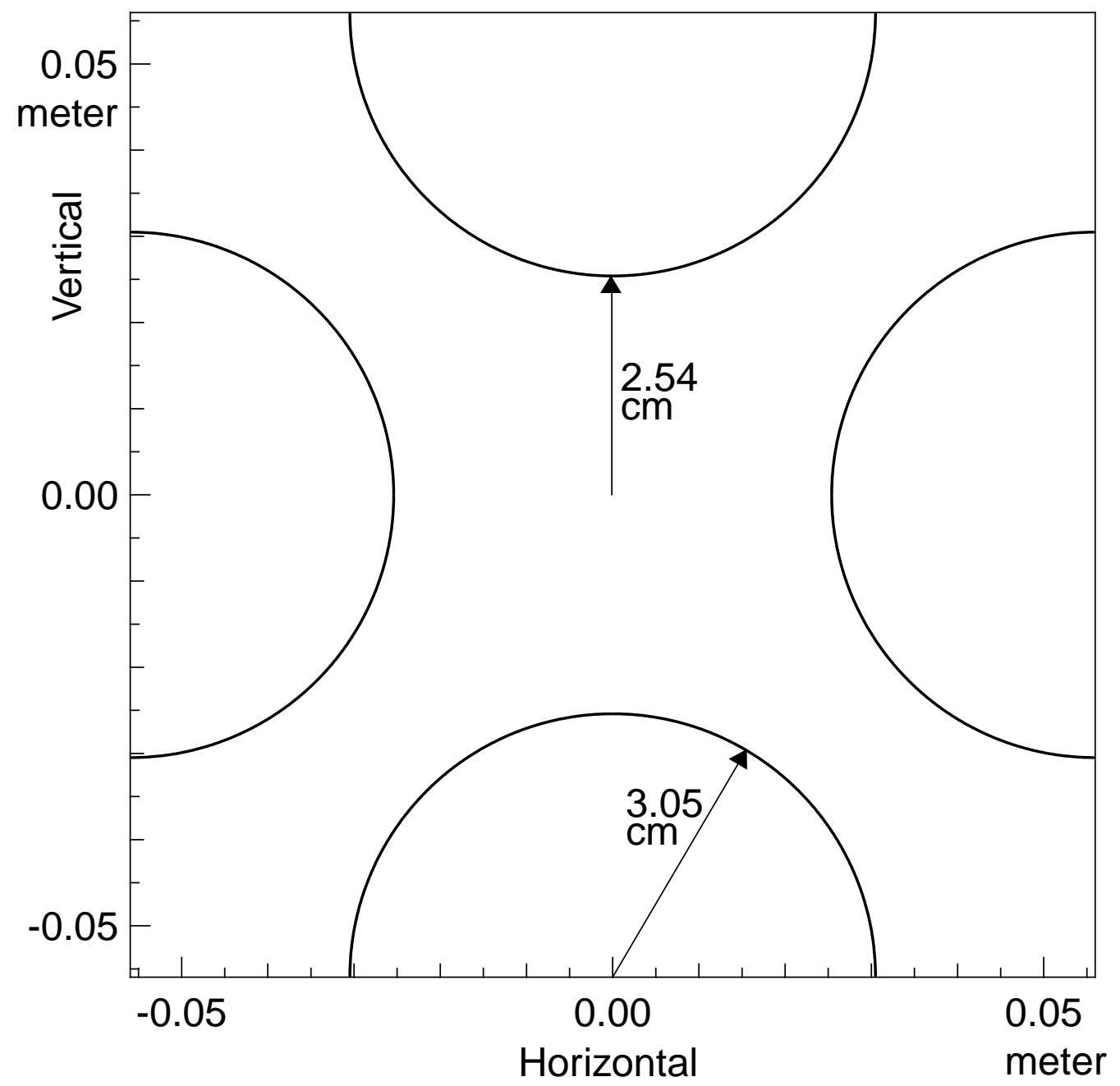

Figure 6.1: The conducting boundary of an electrostatic quadrupole in the Scaled Final Focus Experiment. The area shown corresponds to the grid size used in the simulation. 
presented here. Furthermore, the initial beam distribution was taken to be either K-V or semi-Gaussian, which may not accurately reflect the initial distribution of the beam in the experiment.

\subsection{The Scaled HIBALL-II Case}

For the scaled HIBALL-II case, the focusing strengths of the magnetic quadrupoles in the HIBALL-II design were scaled down to find their corresponding values for the experiment. The focusing strengths thus found were subsequently adjusted in the experiment to minimize the size of the focal spot. Figure 6.2 shows the beam envelope in the Scaled Final Focus Experiment for the scaled HIBALL-II case. It has a beam envelope shape in the magnetic section that corresponds most closely to the HIBALL-II focusing scheme. The purpose of the electrostatic section is to match the beam into the magnetic section, which is the actual scaled HIBALL-II final focus system. Since for this case, the beam radius in the electrostatic section is much smaller than the aperture of the electrostatic quadrupoles, they are not included in the simulation as their effect on the beam will be negligible. This allowed a smaller extent of the grid, and therefore a higher accuracy of the simulation for a given number of grid cells.

In the experiment, the scaled HIBALL-II case was plagued by an anomalously large emittance growth by factors of about six [71]. One of the goals of the simulations of the Scaled Final Focus Experiment was to investigate some of the possible causes of this anomalous emittance growth.

In the experiment, the beam was apertured at D1 in order to get a beam with the 


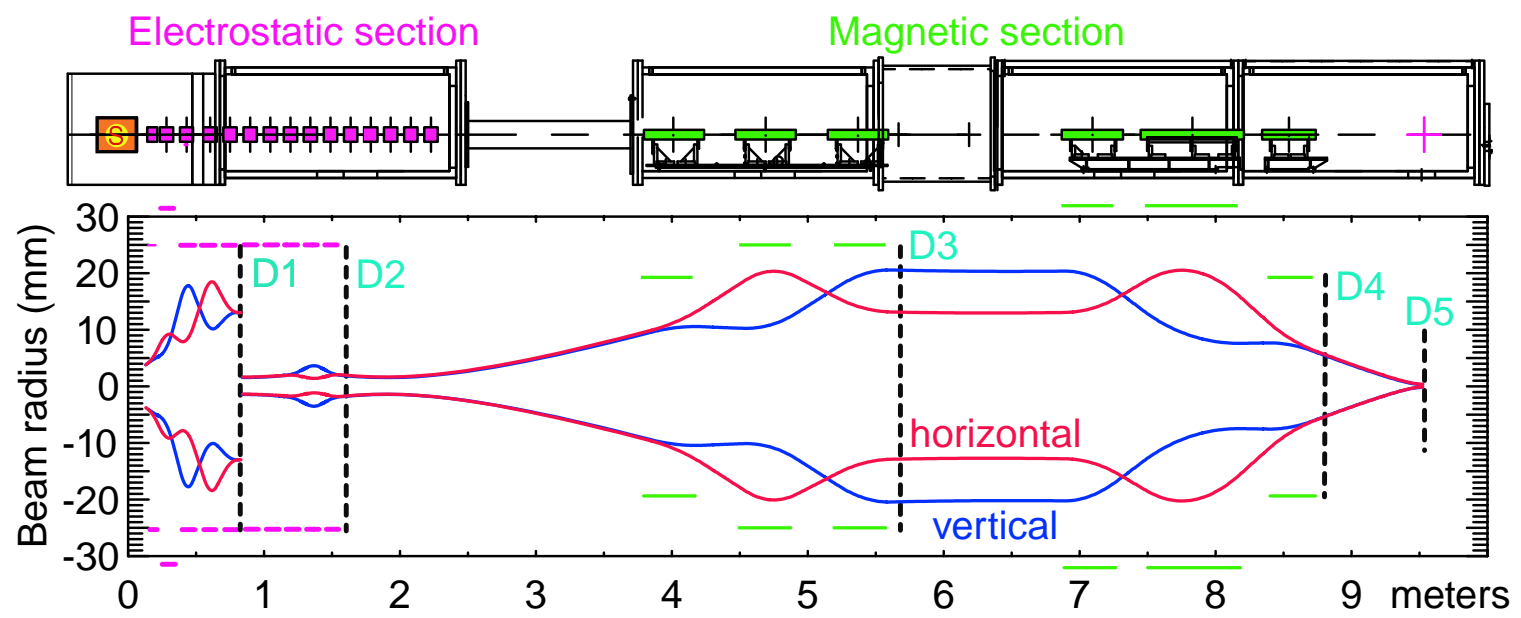

Figure 6.2: The Scaled Final Focus Experiment. The beam envelope shown corresponds to the scaled HIBALL-II case.

appropriate current and emittance. The simulations for the scaled HIBALL-II case started from this aperture, assuming a semi-Gaussian distribution initially. In order to guarantee convergence, several simulations with different numbers of particles, grid cell size, and step size have been performed, with 40,000 particles on a $128 \times 128$ grid with a $1 \mathrm{~cm}$ step size as the baseline case. For the cases that included fringe fields, a $1 \mathrm{~mm}$ step size was used inside the quadrupoles and their fringes. All cases used spatial filtering and fourfold symmetry to solve for the field. The beam parameters are given in table 6.1, whereas table 6.2 lists the focusing lattice properties. Figure 6.3 shows the beam envelope, the extent of the grid, and the location of conducting boundaries in the simulation as a function of the longitudinal distance. The simulations and their results are summarized in table 6.3.

Figure 6.4 shows the horizontal and vertical beam radius calculated from the PIC simulation. Figure 6.5 shows the calculated emittance of an initially semi-Gaussian beam as a function of the longitudinal distance traveled, whereas figure 6.6 shows the calculated emittance for a $\mathrm{K}-\mathrm{V}$ beam. The emittance of an initially semi-Gaussian beam increases by 


\begin{tabular}{|l|l|}
\hline Beam energy & $160 \mathrm{keV}$ \\
\hline Initial edge emittance & $0.996 \pi \mathrm{mm} \mathrm{mrad}$ \\
\hline Beam current & $87 \mu \mathrm{A}$ \\
\hline Initial beam parameters & $a_{0}=b_{0}=1.5 \mathrm{~mm} ; a_{0}^{\prime}=b_{0}^{\prime}=0$. \\
\hline Final focus spot size (from envelope calculation) & $0.207 \mathrm{~mm}^{2}$ \\
\hline
\end{tabular}

Table 6.1: Beam parameters for the scaled HIBALL-II case.

\begin{tabular}{|c|c|c|c|}
\hline $\begin{array}{c}\text { Quadrupole center } \\
\text { locations }(\mathrm{m})\end{array}$ & $\begin{array}{c}\text { Quadrupole } \\
\text { lengths }(\mathrm{m})\end{array}$ & $\begin{array}{c}\text { Field } \\
\text { gradient }\end{array}$ & Aperture $(\mathrm{cm})$ \\
\hline \hline 0.0745 & 0.102 & 0. & 2.54 \\
\hline 0.2275 & 0.102 & $995 \mathrm{kV} / \mathrm{m}^{2}$ & 2.54 \\
\hline 0.3805 & 0.102 & $12592 \mathrm{kV} / \mathrm{m}^{2}$ & 2.54 \\
\hline 0.5335 & 0.102 & $-22909 \mathrm{kV} / \mathrm{m}^{2}$ & 2.54 \\
\hline 0.6865 & 0.102 & $13987 \mathrm{kV} / \mathrm{m}^{2}$ & 2.54 \\
\hline 0.8395 & 0.102 & 0. & 2.54 \\
\hline 0.9925 & 0.102 & 0. & 2.54 \\
\hline 1.1455 & 0.102 & 0. & 2.54 \\
\hline 1.2985 & 0.102 & 0. & 2.54 \\
\hline 1.4515 & 0.102 & 0. & 2.54 \\
\hline 3.3039 & 0.4 & $1.222 \mathrm{~T} / \mathrm{m}$ & 1.91 \\
\hline 4.0034 & 0.4 & $-2.348 \mathrm{~T} / \mathrm{m}$ & 2.54 \\
\hline 4.7069 & 0.4 & $1.365 \mathrm{~T} / \mathrm{m}$ & 2.54 \\
\hline 6.4299 & 0.4 & $1.586 \mathrm{~T} / \mathrm{m}$ & 3.18 \\
\hline 7.1919 & 0.717 & $-1.734 \mathrm{~T} / \mathrm{m}$ & 3.18 \\
\hline 7.9279 & 0.351 & $2.628 \mathrm{~T} / \mathrm{m}$ & 1.91 \\
\hline & & & \\
\hline
\end{tabular}

Table 6.2: Lattice properties for the scaled HIBALL-II case. 


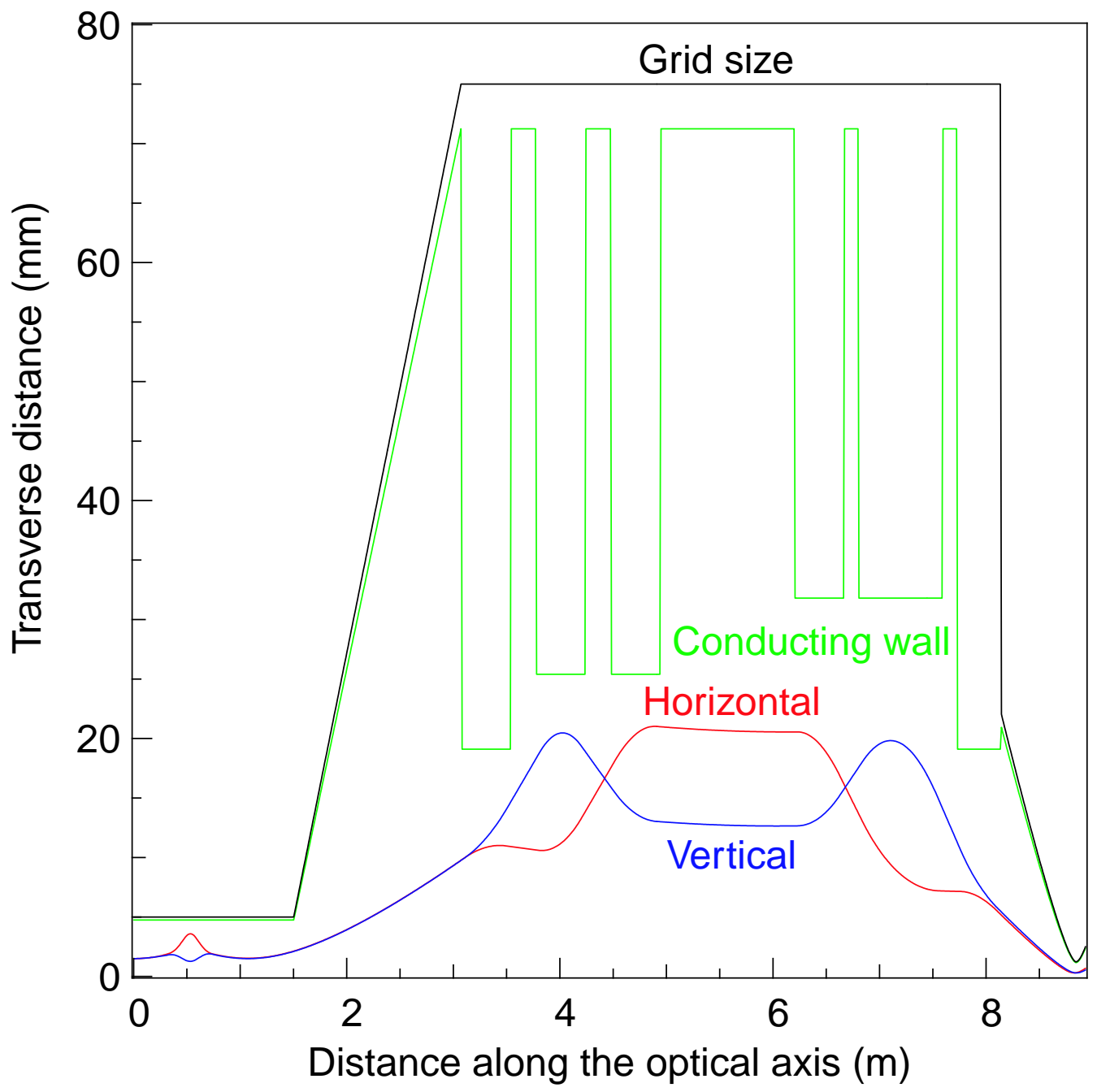

Figure 6.3: The horizontal and vertical beam envelope, extent of the grid, and the location of the conducting boundaries in the simulation of the scaled HIBALL-II case. 


\begin{tabular}{|c|c|c|c|c|c|}
\hline Initial distribution & $\begin{array}{c}\text { Number of } \\
\text { particles }\end{array}$ & Grid size & $\begin{array}{c}\text { Step } \\
\text { size }(\mathrm{cm})\end{array}$ & $\begin{array}{c}\text { Maximum } \\
\text { emittance } \sqrt{\varepsilon_{x} \varepsilon_{y}} \\
(\pi \mathrm{mm} \mathrm{mrad})\end{array}$ & $\begin{array}{c}\text { Spot } \\
\text { area } \\
\left(\mathrm{mm}^{2}\right)\end{array}$ \\
\hline Semi-Gaussian & $\begin{array}{c}40,000 \\
(116 \text { lost })\end{array}$ & $128 \times 128$ & 1. & 1.422 & 0.291 \\
\hline Semi-Gaussian & $\begin{array}{c}80,000 \\
(220 \text { lost })\end{array}$ & $128 \times 128$ & 1. & 1.417 & 0.290 \\
\hline Semi-Gaussian & $\begin{array}{c}10,000 \\
(27 \text { lost })\end{array}$ & $64 \times 64$ & 1. & 1.510 & 0.317 \\
\hline Semi-Gaussian & $\begin{array}{c}160,000 \\
(460 \text { lost })\end{array}$ & $256 \times 256$ & 1. & 1.391 & 0.278 \\
\hline Semi-Gaussian & $\begin{array}{c}40,000 \\
(72 \text { lost })\end{array}$ & $128 \times 128$ & 0.5 & 1.425 & 0.282 \\
\hline K-V & $\begin{array}{c}40,000 \\
(\text { none lost })\end{array}$ & $128 \times 128$ & 1. & 1.026 & 0.202 \\
\hline
\end{tabular}

Table 6.3: Simulation results for the scaled HIBALL-II case.

$42 \%$, while a $\mathrm{K}-\mathrm{V}$ beam suffers no significant emittance increase. Comparing these figures shows that the emittance starts to increase in the drift space between the electrostatic section and the magnetic section, where the beam expands transversely. Note that the emittance decreases sharply as the beam converges onto the focal spot. The initial decrease in the emittance is due to a softening of the hard edge of the semi-Gaussian distribution in configuration space as the initially Gaussian distribution of the transverse velocity is mapped to configuration space. This is a commonly observed feature both in simulations starting with a semi-Gaussian distribution [97] and in experiments [9]. The emittance growth leads to an spot area increase of $55 \%$. We can attribute the emittance growth to space-charge nonlinearities, since it does not occur for a K-V beam, which has a linear space-charge force.

Figures 6.7 and 6.8 show the sheared $x-x^{\prime}$ and $y-y^{\prime}$ phase space of the beam just before the first magnetic quadrupole, at the end of the expansion drift section. From these phase space plots, we see that the emittance increase is not due to a uniform expansion 


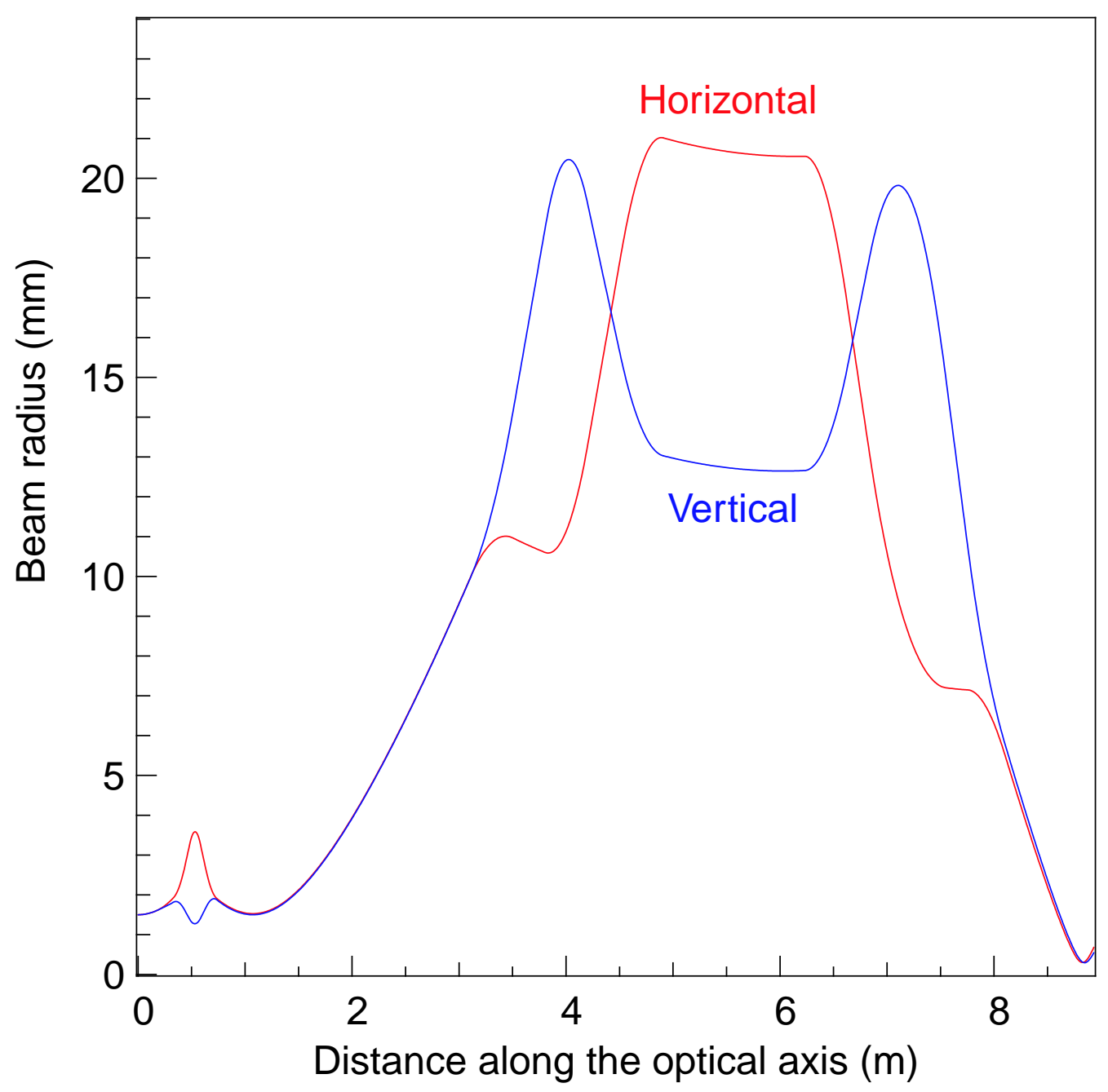

Figure 6.4: The horizontal and vertical beam radius of an initially semi-Gaussian beam as a function of the longitudinal distance traveled, for the scaled HIBALL-II case. 


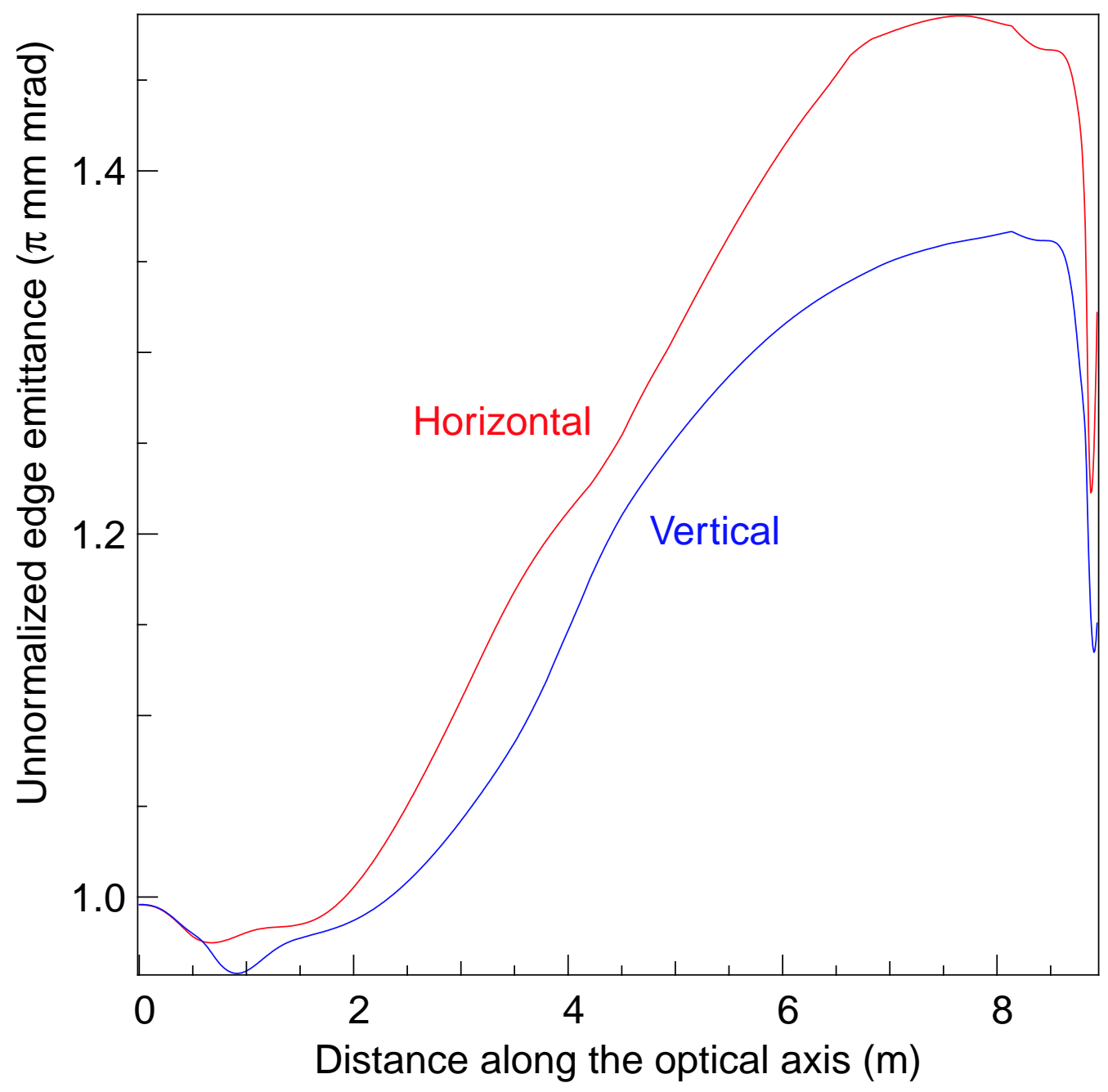

Figure 6.5: The edge emittance of an initially semi-Gaussian beam as a function of the longitudinal distance traveled, for the scaled HIBALL-II case. 


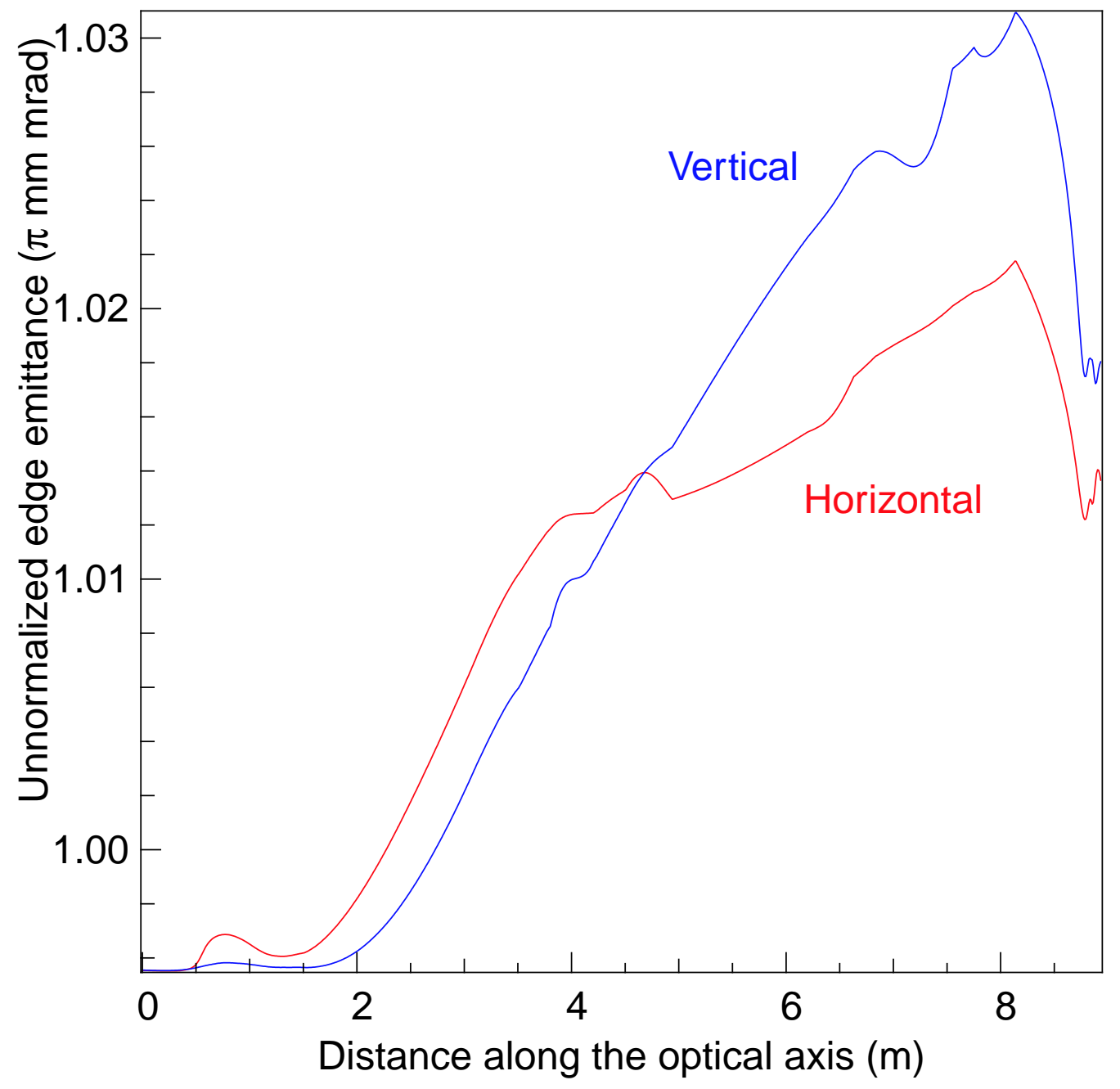

Figure 6.6: The edge emittance of a $\mathrm{K}-\mathrm{V}$ beam as a function of the longitudinal distance traveled, for the scaled HIBALL-II case. 


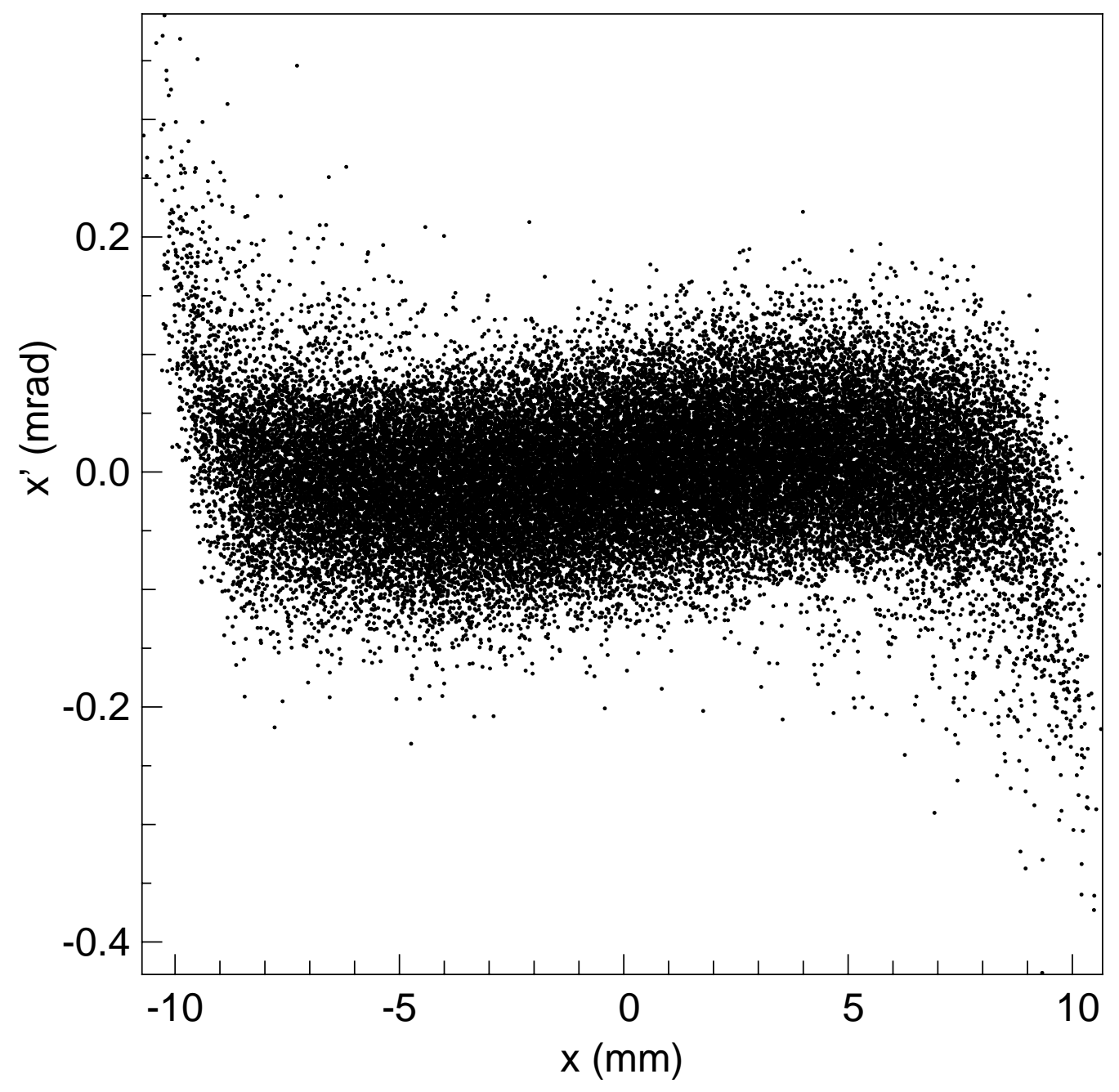

Figure 6.7: Sheared $x-x^{\prime}$ phase space at the end of the expansion drift section, just before the first magnetic quadrupole, for the scaled HIBALL-II case.

of the area of the beam in phase space. Rather, the emittance increase is manifested as distortions near the beam edge.

\subsubsection{Emittance Growth of Expanding Charged Particle Beams}

The emittance growth seen in the simulation is related to the expansion of the beam between the electrostatic and the magnetic section. The emittance growth of a freely 


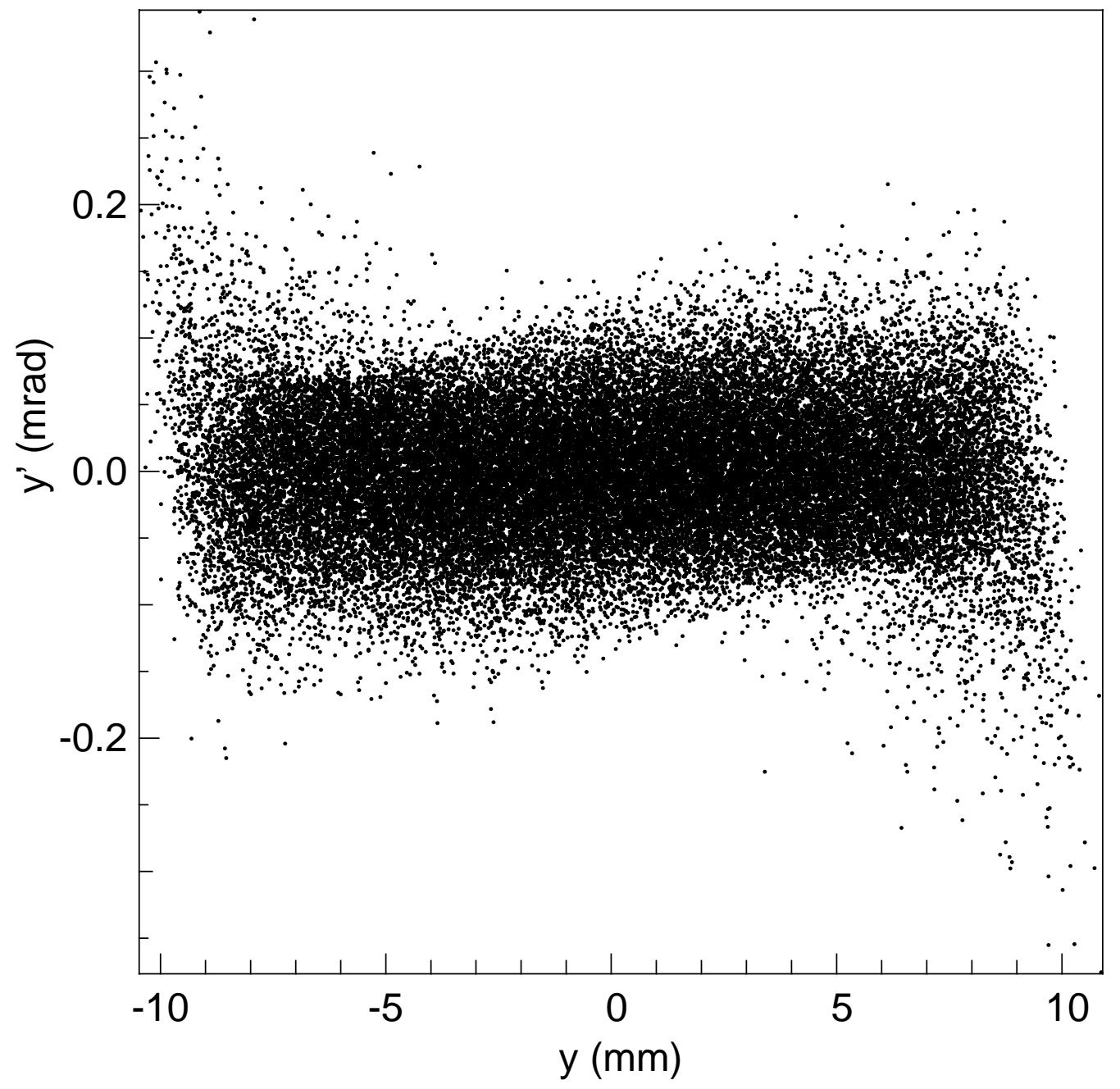

Figure 6.8: Sheared $y-y^{\prime}$ phase space at the end of the expansion drift section, just before the first magnetic quadrupole, for the scaled HIBALL-II case. 
expanding beam can be calculated in general if we resort to dimensionless parameters.

In this derivation, we make use of the paraxial approximation. The equation of motion for a charged particle of mass $m$ and charge $q e$ in a freely expanding beam in this approximation is

$$
m \gamma(\beta c)^{2} \vec{r}^{\prime \prime}=\frac{1}{\gamma^{2}} q e \vec{E}
$$

The electric field $\vec{E}(\vec{r})$ at position $\vec{r}$ can be calculated from Gauss's law as

$$
\vec{E}(\vec{r})=\frac{\lambda}{2 \pi \epsilon_{0}} \int \frac{\vec{r}-\vec{r}_{1}}{\left|\vec{r}-\vec{r}_{1}\right|^{2}} n\left(\vec{r}_{1}\right) \mathrm{d}^{2} \vec{r}_{1},
$$

in which $n(\vec{r})$ is the normalized beam density:

$$
\int n(\vec{r}) \mathrm{d}^{2} \vec{r}=1
$$

The equation of motion then becomes

$$
\vec{r}^{\prime \prime}=Q \int \frac{\vec{r}-\vec{r}_{1}}{\left|\vec{r}-\vec{r}_{1}\right|^{2}} n\left(\vec{r}_{1}\right) \mathrm{d}^{2} \vec{r}_{1}
$$

in which $Q$ is the perveance as defined by equation (2.34).

In order to draw general conclusions about beam expansion, we need to write this equation in dimensionless units. Normalizing the particle position to the initial beam radius $a_{0}$ :

$$
\vec{\rho}=\frac{\vec{r}}{a_{0}}
$$

and defining a dimensionless longitudinal distance $\zeta$ :

$$
\zeta=\frac{z}{a_{0}} \sqrt{Q}
$$

the equation of motion becomes

$$
\frac{\mathrm{d}^{2} \vec{\rho}}{\mathrm{d} \zeta^{2}}=\int \frac{\vec{\rho}-\vec{\rho}_{1}}{\left|\vec{\rho}-\vec{\rho}_{1}\right|^{2}} \nu\left(\rho_{1}\right) \mathrm{d}^{2} \vec{\rho}_{1}
$$


in which the dimensionless transverse density $\nu(\vec{\rho})$ is given by $\nu(\vec{\rho}) \mathrm{d}^{2} \vec{\rho}=n(\vec{r}) \mathrm{d}^{2} \vec{r}$. This equation implies that the distribution of $\vec{\rho}(\zeta)$ depends only on the initial distribution of $\rho$ and $\mathrm{d} \rho / \mathrm{d} \zeta$.

Assuming that the initial distribution of the beam is semi-Gaussian:

$$
f_{0}\left(\vec{r}, \vec{r}^{\prime}\right)=\frac{2}{\pi^{2} \varepsilon_{0}^{2}} \theta\left(1-\frac{\vec{r}^{2}}{a_{0}^{2}}\right) \exp \left[-\frac{1}{2}\left(\frac{2 a_{0} \vec{r}^{\prime}}{\varepsilon_{0}}\right)^{2}\right]
$$

in which $\varepsilon_{0}$ is the initial edge emittance of the beam, the initial distribution in its normalized form depends on one independent parameter $\mu$ only:

$$
\phi_{0}\left(\vec{\rho}, \frac{\mathrm{d} \vec{\rho}}{\mathrm{d} \zeta}\right)=\frac{\mu^{2}}{2 \pi^{2}} \theta\left(1-\vec{\rho}^{2}\right) \exp \left[-\frac{1}{2} \mu^{2}\left(\frac{\mathrm{d} \vec{\rho}}{\mathrm{d} \zeta}\right)^{2}\right]
$$

in which $\mu$ is given by

$$
\mu=\frac{2 a_{0}}{\varepsilon_{0}} \sqrt{Q}
$$

This parameter describes the ratio between the self-repulsion of the beam due to its space charge, and the beam pressure due to its emittance.

The initial beam distribution represents a one-parameter family of initial conditions, whereas the equation of motion (6.7) does not contain any explicit parameters. We conclude that the beam distribution in dimensionless units at longitudinal distance $\zeta$, if semi-Gaussian initially, will depend on the parameter $\mu$ only.

We can estimate the beam radius and emittance from second order moments over the distribution function. The beam radius $a(z)$ is given by

$$
a=\sqrt{2\left\langle\vec{r}^{2}\right\rangle} .
$$

in which the factor $\sqrt{2}$ is chosen such that $a(z=0)=a_{0}$. In dimensionless units, we can 
write this as

$$
\alpha \equiv \frac{a}{a_{0}}=\sqrt{2\left\langle\vec{\rho}^{2}\right\rangle}
$$

The edge emittance for a cylindrically symmetric beam can be calculated as

$$
\varepsilon^{2}=4\left[\left\langle\vec{r}^{2}\right\rangle\left\langle\vec{r}^{\prime 2}\right\rangle-\left\langle\vec{r} \cdot \vec{r}^{\prime}\right\rangle^{2}\right]
$$

In normalized units, we can write this as

$$
\frac{\varepsilon}{\varepsilon_{0}}=\mu \sqrt{\left\langle\vec{\rho}^{2}\right\rangle\left\langle\left(\frac{\mathrm{d} \vec{\rho}}{\mathrm{d} \zeta}\right)^{2}\right\rangle-\left\langle\vec{\rho} \cdot \frac{\mathrm{d} \vec{\rho}}{\mathrm{d} \zeta}\right\rangle^{2}} .
$$

Since the equation of motion, and therefore the phase space density, depends on the longitudinal distance $\zeta$ and the parameter $\mu$ only, the right-hand-side of this equation is a function of those two parameters only:

$$
\frac{\varepsilon}{\varepsilon_{0}}=F(\zeta ; \mu)
$$

We can find the function $F(\zeta ; \mu)$ by performing particle-in-cell simulations of expanding semi-Gaussian beams for different values for the parameter $\mu$ and plotting the relative emittance increase. For $\mu$ much larger than unity, the beam is cold, leading to self-similar expansion, for which the emittance is constant [65]. For $\mu$ much smaller than unity, the space-charge self-repulsion is small compared to the internal beam pressure (emittance), so the nonlinear space-charge forces driving the emittance growth are also small. We expect the emittance to increase significantly for $\mu$ of order unity.

Figure 6.9 shows the emittance increase as a function of the normalized distance $\zeta$ as calculated by WARPxy. Again the initial decrease in the RMS emittance is due to a softening of the hard edge of the semi-Gaussian distribution in configuration space, and can 
be avoided by allowing the semi-Gaussian beam to relax in an alternating-gradient focusing lattice before expansion.

Unlike a K-V distribution, for which the emittance remains constant, the emittance of an initially semi-Gaussian beam increases linearly for large $\zeta$. No saturation of the emittance increase is observed. The linear part of the emittance increase can be parameterized as

$$
\frac{\mathrm{d} \varepsilon / \varepsilon_{0}}{\mathrm{~d} \zeta}=0.114 \exp \left[-\left(\frac{-1.52+\ln \mu}{2.07}\right)^{2}\right] .
$$

This function is drawn in figure 6.10, together with the slopes found in the simulations for different values of $\mu$.

The transverse Debye length of a beam with particle density $n$ at temperature $T$ is given by

$$
\lambda_{\mathrm{D}}=\gamma \sqrt{\frac{\epsilon_{0} T}{n(q e)^{2}}}=\frac{\varepsilon}{2 \sqrt{Q}} .
$$

In terms of the initial beam radius $a_{0}$, we can write this as

$$
\frac{\lambda_{\mathrm{D}}}{a_{0}}=\frac{1}{\mu} \frac{\varepsilon}{\varepsilon_{0}}=\frac{F(\zeta ; \mu)}{\mu}
$$

using equation (6.15). Again this ratio depends on the parameters $\mu$ and $\zeta$ only. We can use the Debye length in normalized units as a scale length to describe distortions in phase space that occur in expanding charged particle beams.

Figure 6.11 shows the sheared phase space of an expanding beam, initially semiGaussian, with $\mu=5.0$ after traveling a longitudinal distance $\zeta=10.0$. In sheared phase space, the particle divergence angles are corrected for the average beam divergence angle at the particle location:

$$
\delta\left(\frac{\mathrm{d} \rho}{\mathrm{d} \zeta}\right)=\frac{\mathrm{d} \rho}{\mathrm{d} \zeta}-\frac{\mathrm{d} \alpha}{\mathrm{d} \zeta} \frac{\rho}{\alpha}
$$




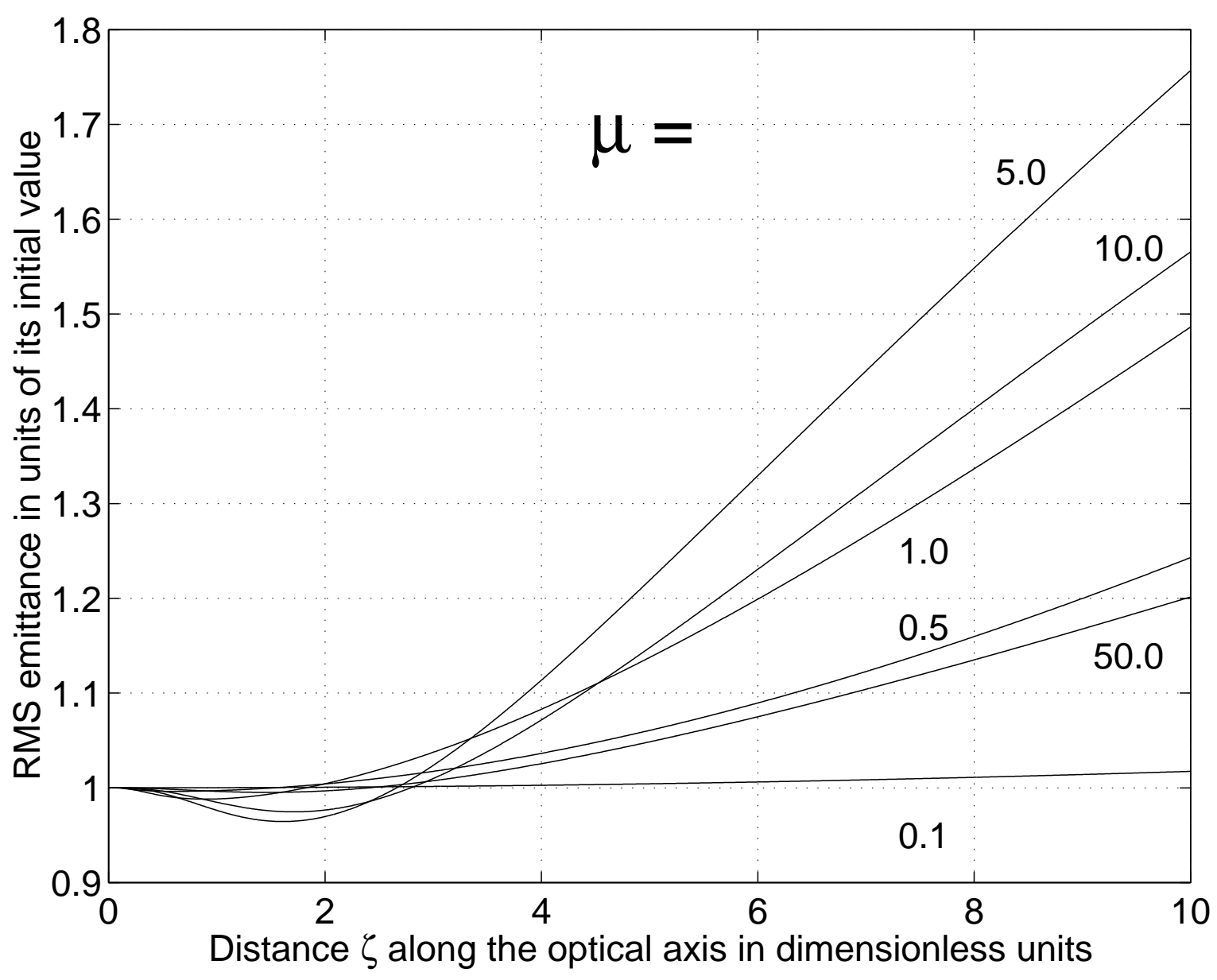

Figure 6.9: Relative emittance increase $F(\zeta ; \mu)$ in WARPxy particle-in-cell simulations of expanding beams, using 40,000 macroparticles on a $128 \times 128$ spatial grid with fourfold symmetry, and 100 time steps. The beam current was $87 \mu \mathrm{A}$, the beam energy $160 \mathrm{keV}$, the initial beam radius was $1.499 \mathrm{~mm}$, while the initial emittance is chosen to match the values of $\mu$ shown for each case. The initial distribution is semi-Gaussian. 


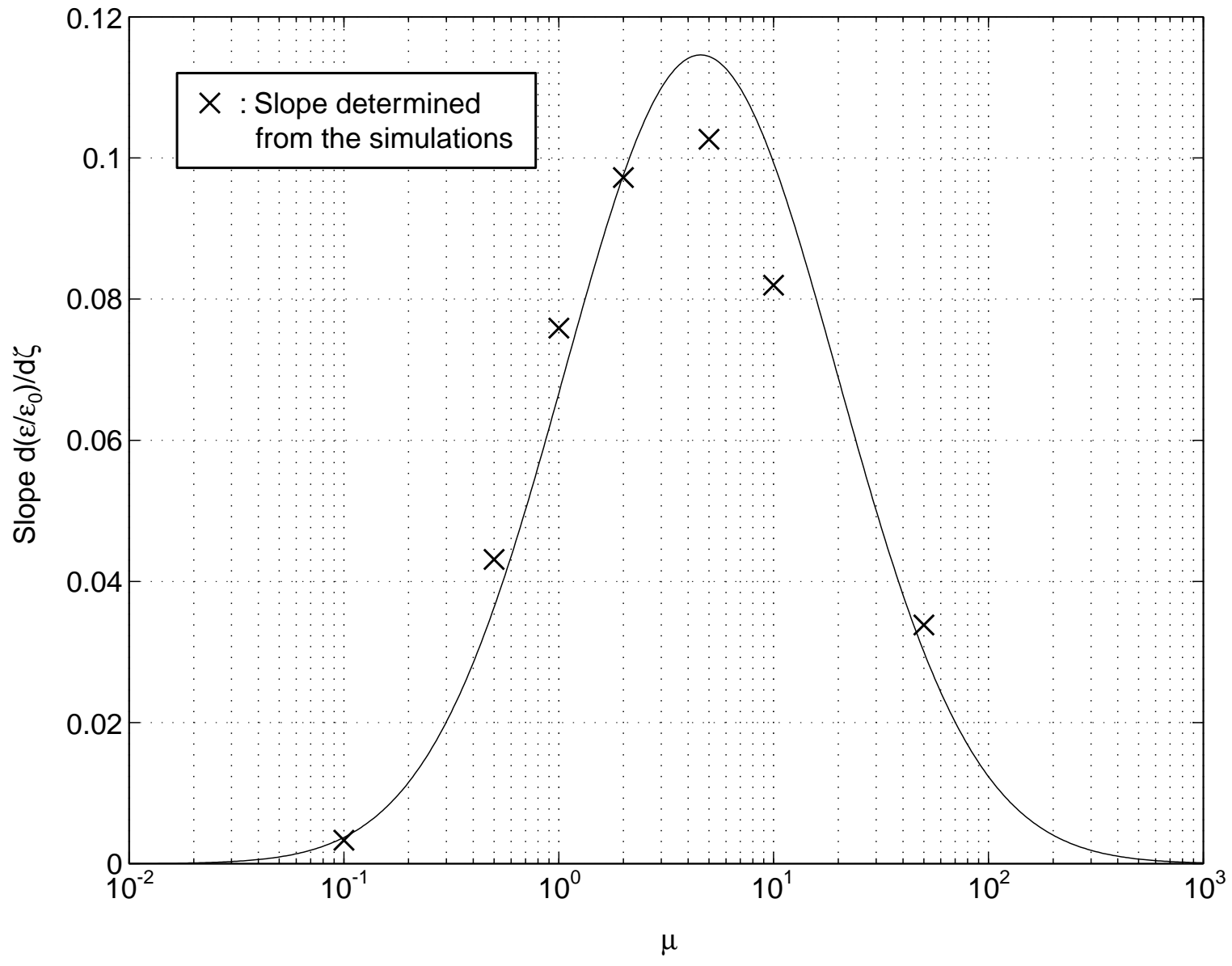

Figure 6.10: The slope $\partial F(\zeta ; \mu) / \partial \zeta$ of the linear part of the emittance increase in figure 6.9 as a function of $\mu$, as parameterized by equation (6.16), as well as the slopes found in the simulation for different values of $\mu$. 
The sheared phase space plot shows that the emittance increase observed occurs near the beam edge. Due to the Gaussian tail that develops in the particle distribution in configuration space, less current is enclosed near the beam edge than in case of a uniform beam. The repulsive space-charge force near the beam edge is therefore smaller than in case of a uniform beam, causing particles there to have a comparatively smaller expansion velocity. As a result, the ellipse in phase space becomes distorted as shown in figure 6.11. The width of the tail that develops in sheared phase space is several Debye lengths wide. Since $F(\zeta ; \mu)$ is a linear function of $\zeta$ for large $\zeta$, whereas the beam radius $\alpha$ increases faster than linear, we conclude from equation (6.18) that the Debye length and therefore the tail width decrease with respect to the beam radius during expansion.

In the case shown in figure 6.11 , the tail that is formed in the expansion contains about $10 \%$ of the beam particles. To assess its effect on the final spot size, we changed the sign of the beam divergence to let the beam converge to a spot. Changing the sign of the beam divergence does not alter the phase space plot as shown in figure 6.11. After letting the beam converge to a spot, it turned out that $76.3 \%$ of the particles were inside the initial beam radius $a_{0}$, as compared to $98.4 \%$ for a $\mathrm{K}-\mathrm{V}$ beam. So we conclude that the tail formed in the phase space of an expanding charged-particle beam has a significant effect on the achievable spot size.

\subsubsection{Fringe Field Effects}

Although the simulations indicate that a significant emittance increase will occur due to space-charge nonlinearities, the emittance increase found in the experiment is much larger. Since no significant emittance increase was found in simulations starting with a K-V 


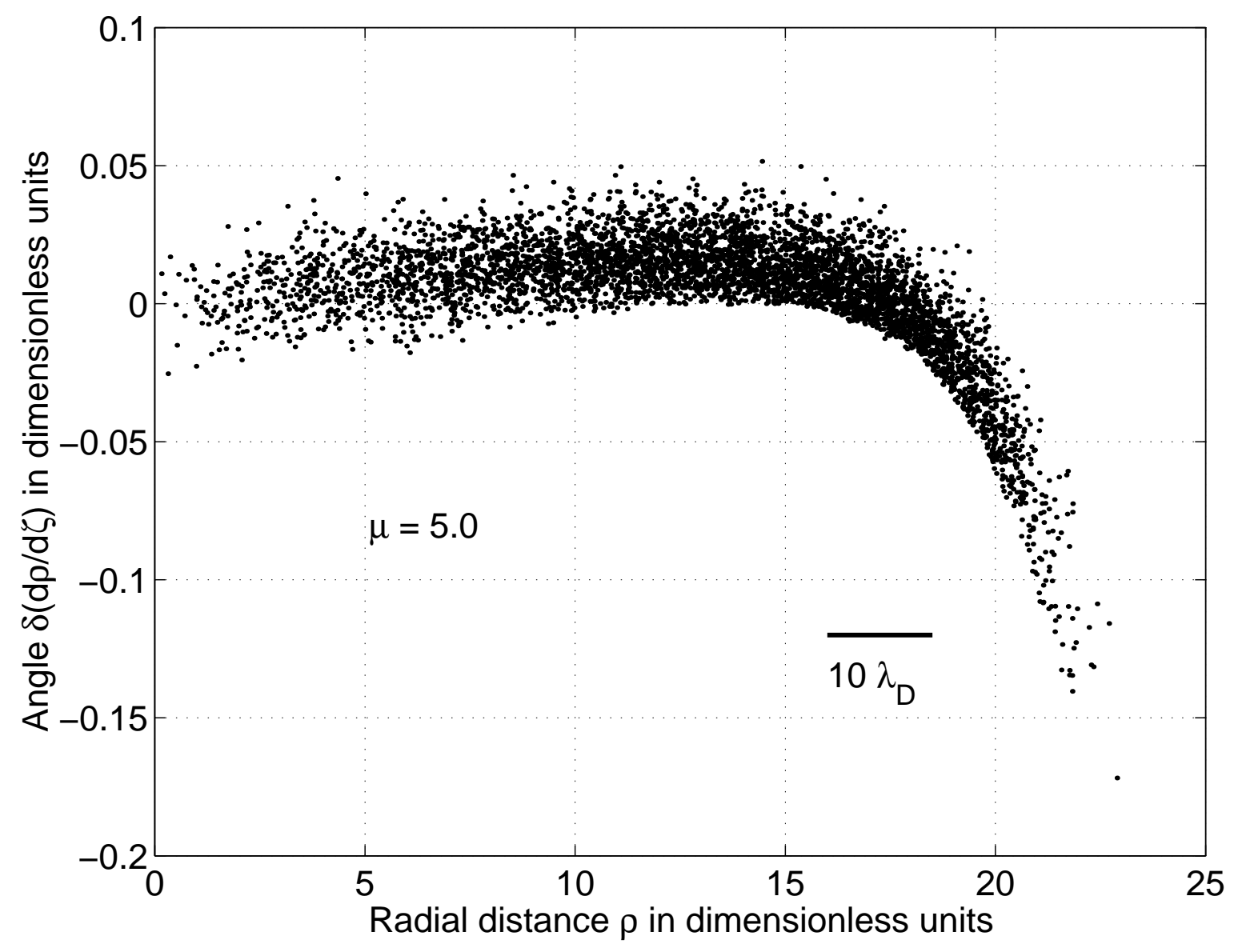

Figure 6.11: Sheared phase space of an expanding beam with $\mu=5.0$ after traveling a longitudinal distance $\zeta=10.0$ in dimensionless units. The radial distance and the angle are both shown in dimensionless units. This simulation was done using the WARPxy particlein-cell code, using 40,000 macroparticles on a $128 \times 128$ spatial grid with fourfold symmetry, and 100 time steps. The beam current was $87 \mu \mathrm{A}$, the beam energy $160 \mathrm{keV}$, the initial beam radius was $1.499 \mathrm{~mm}$, while the initial emittance was $1.91 \pi \mathrm{mm}$ mrad, such that $\mu=5.0$. The initial distribution is semi-Gaussian. 
distribution, it is unlikely that nonlinear image charges (which were already included in these simulations) play a significant role. Third-order aberrations in the form of nonlinear fringe fields represent another possible cause of emittance growth.

In the simulations presented here, the fringe fields were assumed to have the shape shown in figure 2.1, centered around the nominal magnet ends, with a fringe length equal to the element apertures given in table 6.2. As described in section 2.4.1, the lowest-order nonlinearities in fringe fields generated by the longitudinal variation of the quadrupolar field consist of a pseudo-octupole field and a longitudinal field. For the electrostatic/magnetic quadrupoles, these components correspond in equations $(2.9)-(2.11) /(2.12)-(2.14)$ to the $e / b_{2,1}(z)$ term and the $e / b_{2,0}^{\prime}(z)$ term respectively, where the prime denotes a derivative with respect to the longitudinal coordinate $z$.

Figure 6.12 shows the emittance as a function of the longitudinal distance traveled for a beam with an initially semi-Gaussian distribution, if we include both the pseudooctupole field $e / b_{2,1}(z)$ and the longitudinal field $e / b_{2,0}^{\prime}(z)$. We see that spikes occur in the emittance, in which the emittance rapidly increases and equally fast decreases by approximately the same amount. In those locations, the $x-x^{\prime}$ and $y-y^{\prime}$ phase space is heavily distorted into an s-shape, and rapid changes in the width of s-shape result in significant, though transient, changes in the emittance. The presence of nonlinear fringe fields causes a negligible increase in the overall emittance growth. We find a similar effect for a beam that starts with a K-V distribution (figure 6.13). Again short spikes occur in the emittance, but the overall emittance increase is small as in the case without fringe fields.

At first sight, it may be surprising that the phase space area would increase so 


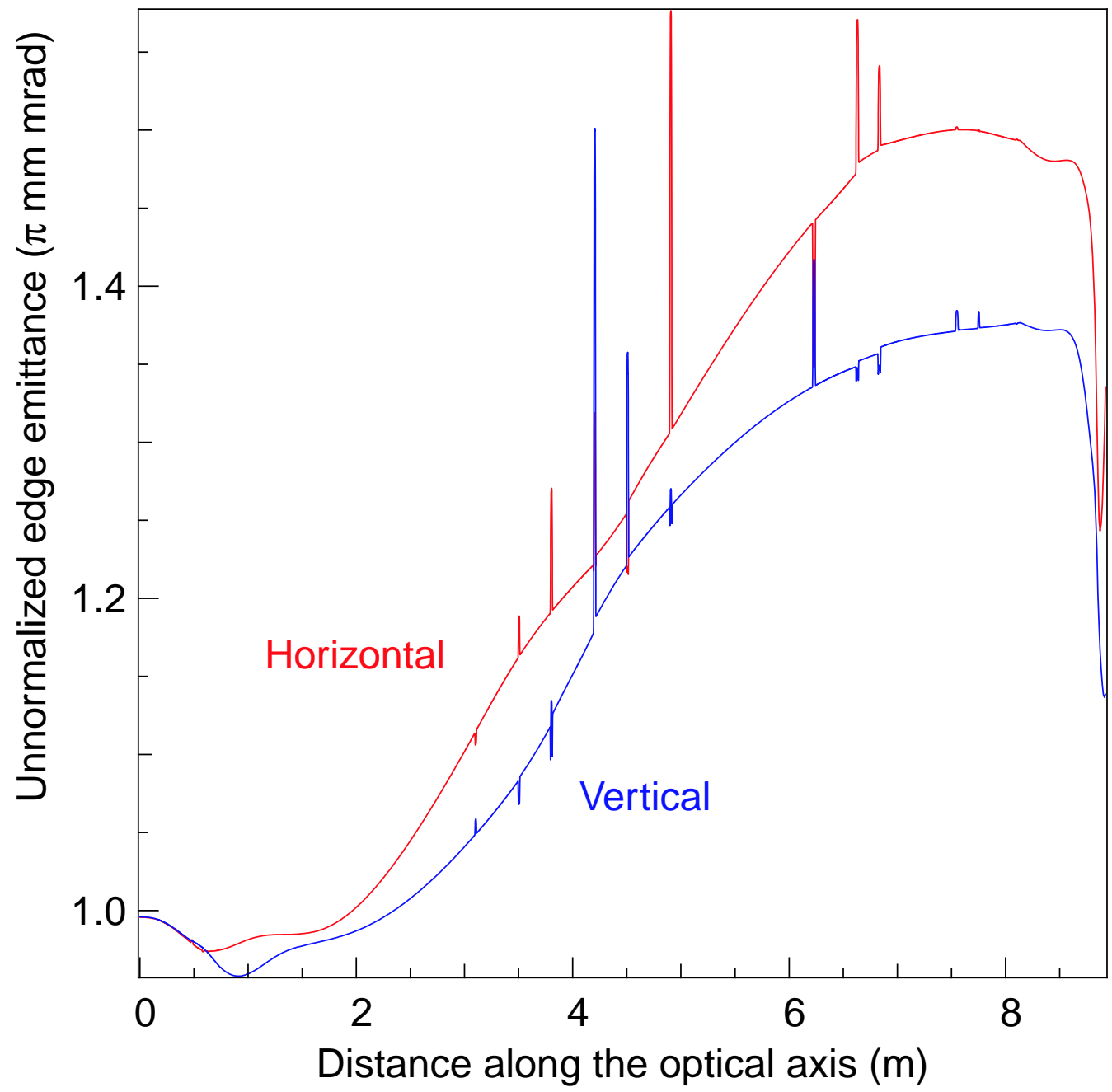

Figure 6.12: The edge emittance of an initially semi-Gaussian beam as a function of the longitudinal distance traveled, for the scaled HIBALL-II case. This simulation included both the pseudo-octupole $e / b_{2,1}(z)$ and the longitudinal field $e / b_{2,0}^{\prime}(z)$ caused by the fringe fields of the electrostatic/magnetic quadrupoles. 


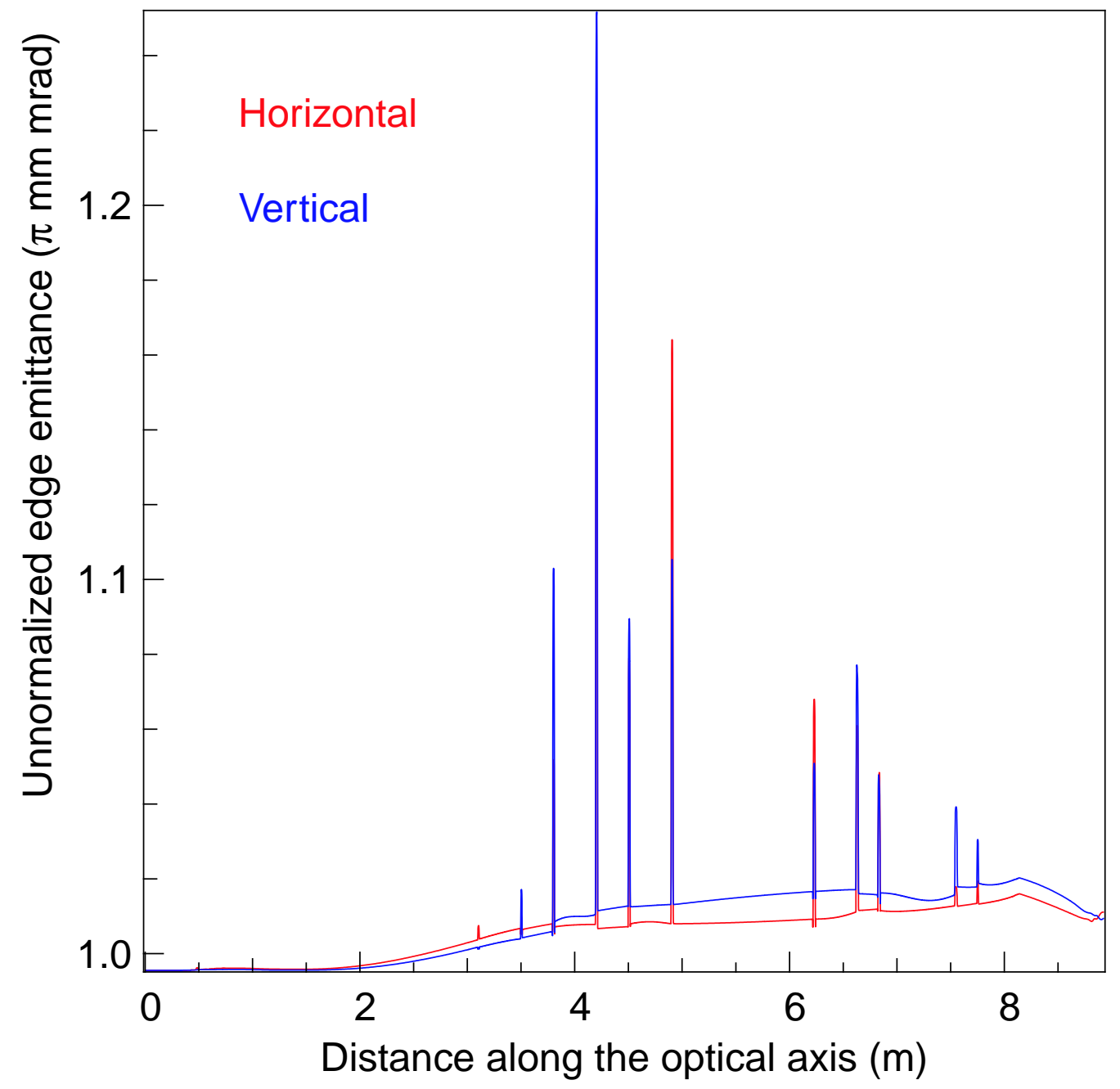

Figure 6.13: The edge emittance of an initially K-V beam as a function of the longitudinal distance traveled, for the scaled HIBALL-II case. This simulation included both the pseudooctupole $e / b_{2,1}(z)$ and the longitudinal $e / b_{2,0}^{\prime}(z)$ field caused by the fringe fields of the electrostatic/magnetic quadrupoles. 
rapidly in the fringe fields. However, as the beam enters the fringe fields of the focusing quadrupoles, its phase space is severely distorted from the initial elliptical shape. The edge emittance, which is an RMS quantity, is therefore no longer a good measure of the phase space area of the beam.

The spikes in the emittance are caused by the pseudo-octupole component of the fringe field. Figure 6.14 shows the emittance of an initially semi-Gaussian beam if we include the longitudinal field $e / b_{2,0}^{\prime}(z)$ in the fringes but not the pseudo-octupole field $e / b_{2,1}(z)$. Although the overall emittance is still somewhat larger than in the case without fringe fields, the spikes in the emittance have disappeared.

Note that in equation $(2.70)$, the pseudo-octupole term $e / b_{2,1}(z)$ depends on the second derivative with respect to $z$ of the quadrupolar field, whereas in equation (2.69) the longitudinal term $e / b_{2,0}^{\prime}(z)$ depends on the first derivative. From figure 2.1, we see that then the longitudinal field $e / b_{2,0}^{\prime}(z)$ at the end of a quadrupole consists of one peak with a width of the order of the fringe length, while the pseudo-octupole consists of two peaks with opposite signs, separated by the length of the fringe. These two peaks cause the rapid increase and decrease in the emittance in the fringe fields of the quadrupoles. As an example, figure 6.15 shows the magnitude of the pseudo-octupole field $b_{2,1}(z)$ at the end of the third magnet, together with the horizontal and vertical emittances.

We can conclude that the fringe fields are unlikely to be the cause of the anomalous emittance growth found in the experiment. It may be that the anomalous emittance growth is linked to the rotation of the beam that was found in the experiment. This will be discussed in section 6.4. 


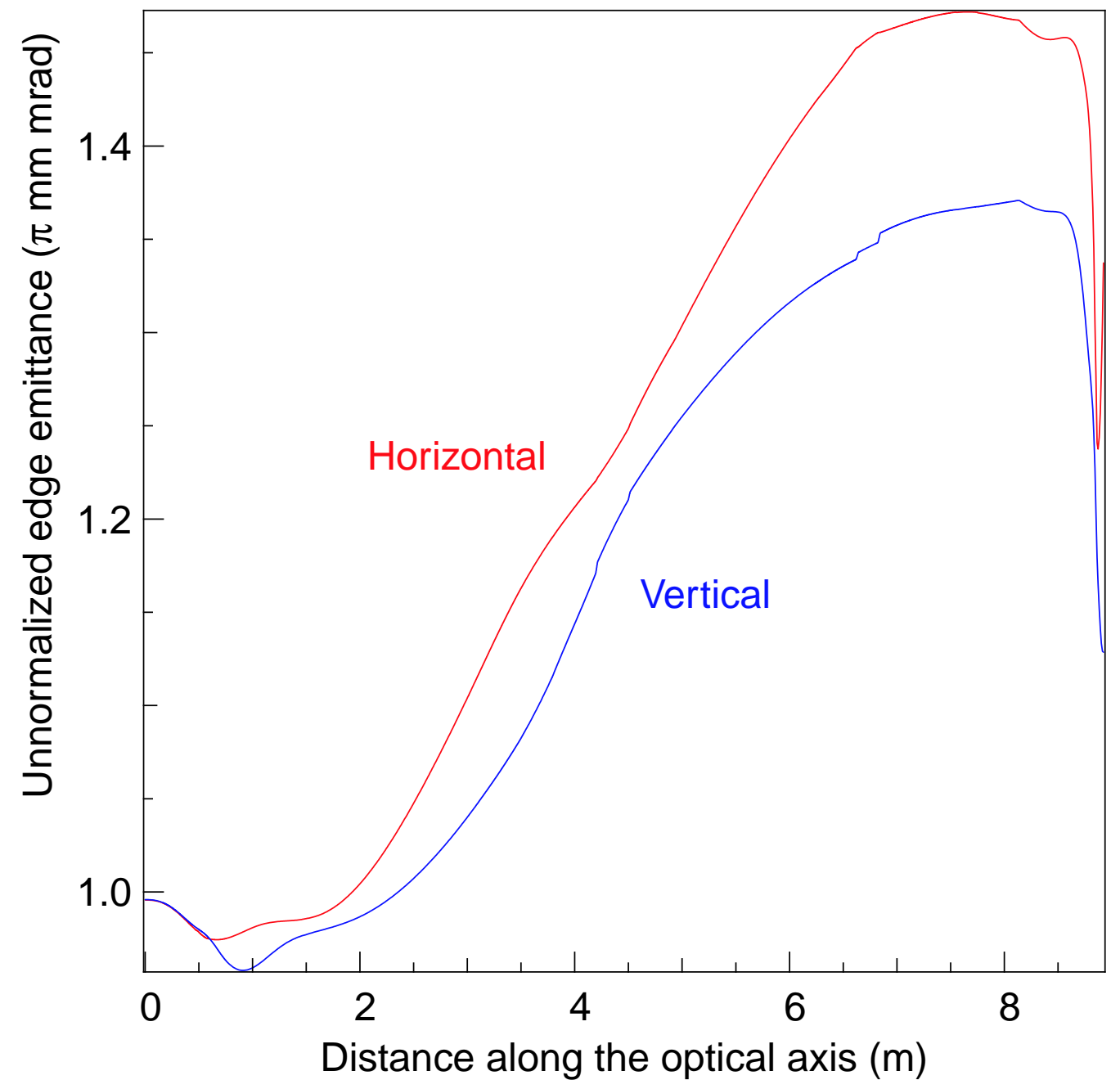

Figure 6.14: The edge emittance of an initially semi-Gaussian beam as a function of the longitudinal distance traveled, for the scaled HIBALL-II case. This simulation included only the longitudinal field $e / b_{2,0}^{\prime}(z)$ caused by the fringe fields of the electrostatic/magnetic quadrupoles. 


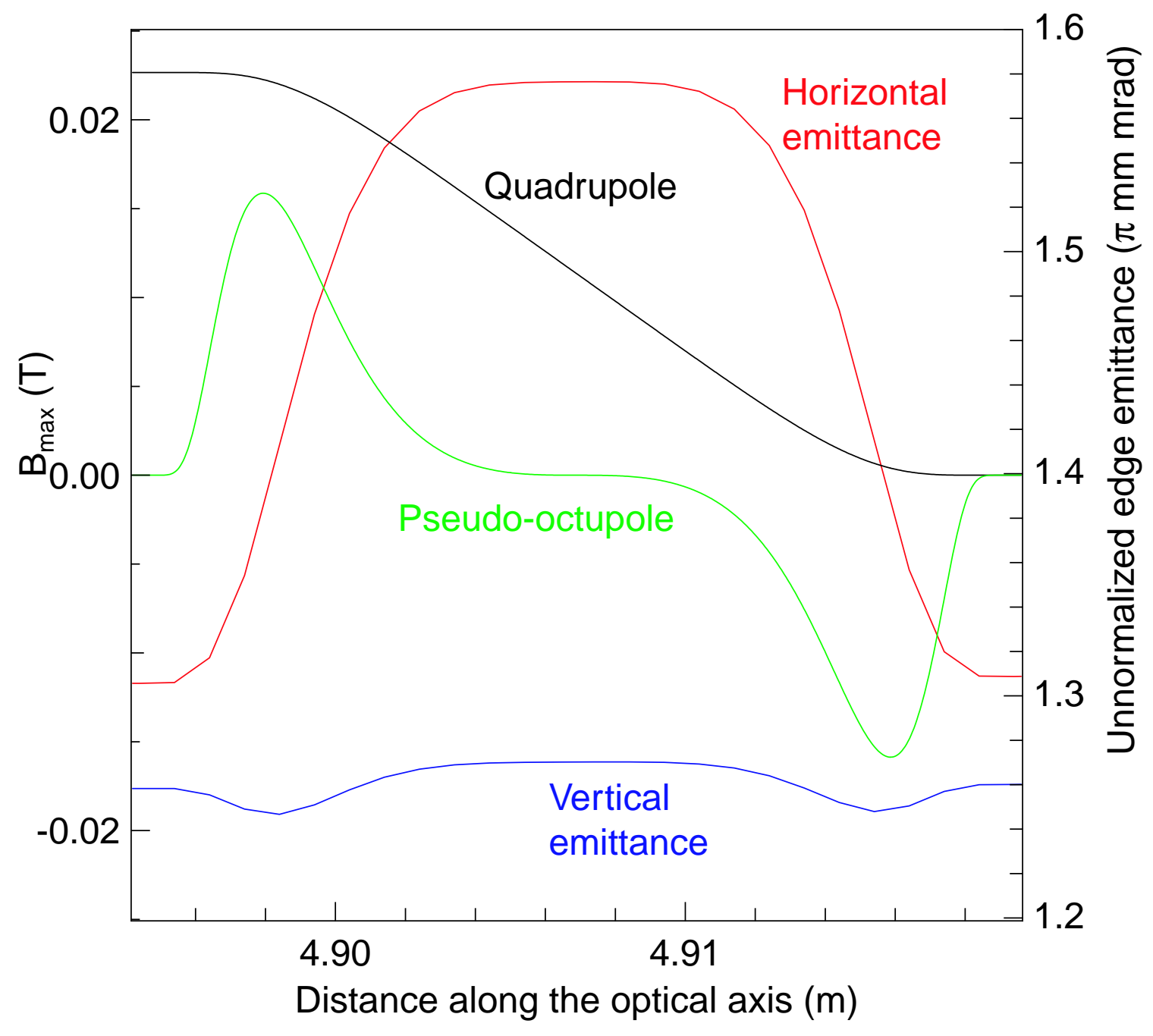

Figure 6.15: The magnitude of the quadrupole and the pseudo-octupole component of the fringe field $\left(b_{2,0}(z) r\right.$ and $\left.2 b_{2,1}(z) r^{3}\right)$ at the end of the third magnet as a function of position, together with the edge emittance of an initially semi-Gaussian beam. The quadrupole and pseudo-octupole field are evaluated at the beam radius $r=1.66 \mathrm{~cm}$. 


\subsection{The Modified Scaled HIBALL-II Case}

For the scaled HIBALL-II case discussed above, the simulations indicated that a sizeable emittance increase would occur due to the transverse expansion of the beam in the experiment. In addition, an anomalous emittance growth was measured experimentally for the scaled HIBALL-II case. An alternative envelope solution was therefore investigated in the experiment [72]. This envelope solution is shown in figure 6.16. A beam with a current of $95 \mu \mathrm{A}$ was used. In this modified scaled HIBALL-II case, the beam is not apertured at diagnostic station D1 in the experiment, unlike the scaled HIBALL-II case discussed above. This eliminates one of the possible causes of the anomalous emittance growth. No anomalous emittance increase was observed in the experiment for this case.

The simulations for the modified scaled HIBALL-II case started at the injector, assuming a semi-Gaussian distribution initially. In the experiment, the initial emittance at the source is unknown. In the simulation, its value was chosen such that the emittance at D1 in the simulation matches the measured emittance at D1 in the experiment. Since the beam is much smaller than the aperture in the first couple of electrostatic quadrupoles, their image field effects are negligible. The presence of the conducting boundaries of the first five quadrupoles was therefore not included in the simulation; they were replaced by circular conducting boundaries at a distance of about four times the beam radius. Again several simulations with different numbers of particles, grid cell size, and step size were performed in order to guarantee convergence, with 40,000 particles on a $256 \times 256$ grid with a $1 \mathrm{~cm}$ step size as the baseline case. All cases used spatial filtering and fourfold symmetry to solve for the field. The beam parameters are given in table 6.4, whereas table 6.5 lists 


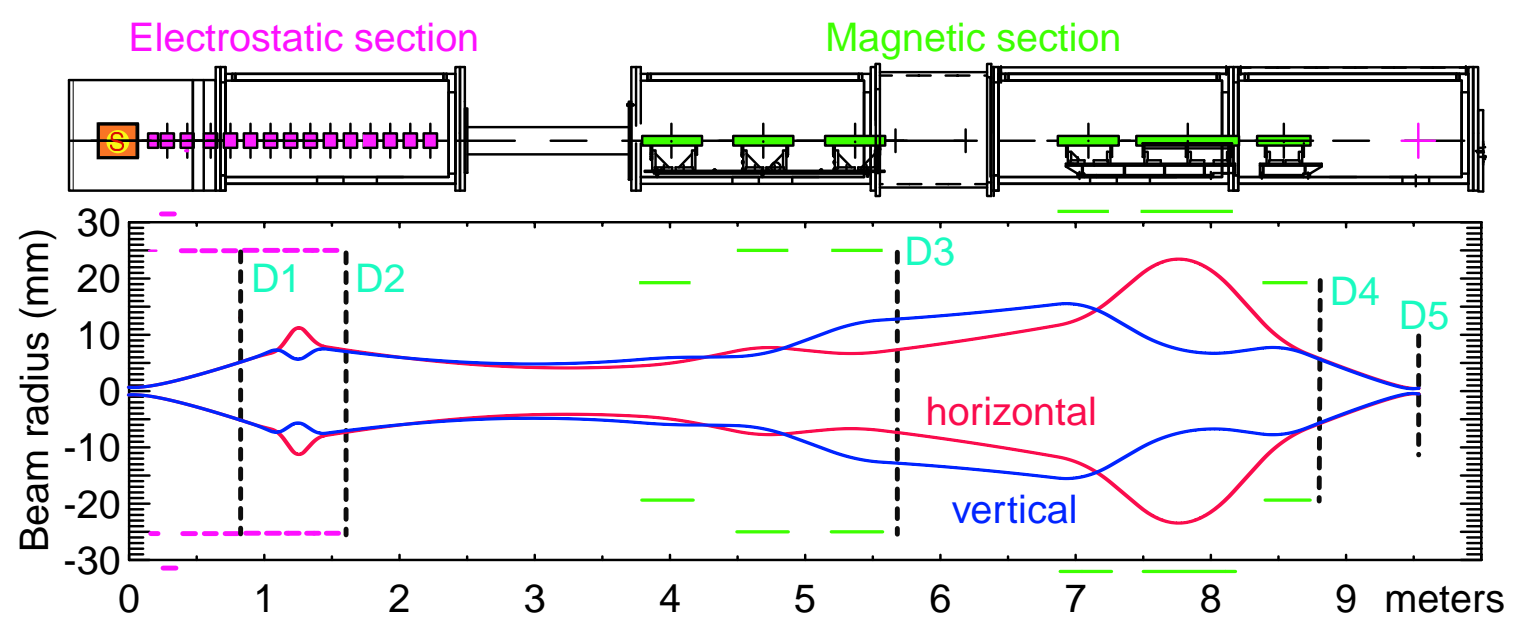

Figure 6.16: The Scaled Final Focus Experiment. The beam envelope shown corresponds to the modified scaled HIBALL-II case.

\begin{tabular}{|l|l|}
\hline Beam energy & $160 \mathrm{keV}$ \\
\hline Initial edge emittance & $2.25 \pi \mathrm{mm} \mathrm{mrad}$ \\
\hline Beam current & $95 \mu \mathrm{A}$ \\
\hline Initial beam parameters & $\begin{array}{l}a_{0}=b_{0}=0.67 \mathrm{~mm} ; \\
a_{0}^{\prime}=b_{0}^{\prime}=-1.0 \mathrm{mrad}\end{array}$ \\
\hline Final focus spot size (from envelope calculation) & $0.589 \mathrm{~mm}^{2}$ \\
\hline
\end{tabular}

Table 6.4: Beam parameters for the modified scaled HIBALL-II case.

the focusing lattice properties. Figure 6.17 shows the beam envelope, the extent of the grid, and the location of conducting boundaries in the simulation as a function of the longitudinal distance. The simulations and their results are summarized in table 6.6.

From the data in table 6.4 and 6.6, we find that the emittance increases by a factor of about 1.9. The emittance is shown as a function of distance along the optical axis in figure 6.18. Comparing this figure to the beam radii shown in figure 6.19 , we see that emittance changes typically coincide with changes in the beam radius. In case the initial beam distribution is $\mathrm{K}-\mathrm{V}$ instead of semi-Gaussian, no significant emittance increase occurs. These results agree with our conclusions of section 6.1 , where we found that the emittance 


\begin{tabular}{|c|c|c|c|}
\hline $\begin{array}{l}\text { Quadrupole center } \\
\text { locations }(\mathrm{m})\end{array}$ & $\begin{array}{l}\text { Quadrupole } \\
\text { lengths (m) }\end{array}$ & $\begin{array}{c}\text { Field } \\
\text { gradient }\end{array}$ & Aperture $(\mathrm{cm})$ \\
\hline $\begin{array}{ll}0.0651 \\
\end{array}$ & 0.0762 & 0 . & 2.54 \\
\hline 0.1730 & 0.1016 & 0 . & 2.54 \\
\hline 0.3254 & 0.1016 & 0. & 2.54 \\
\hline 0.5041 & 0.1016 & 0 . & 2.54 \\
\hline 0.6565 & 0.1016 & 0 . & 2.54 \\
\hline 0.8089 & 0.1016 & 0. & 2.54 \\
\hline 0.9613 & 0.1016 & $-1001 \mathrm{kV} / \mathrm{m}^{2}$ & 2.54 \\
\hline 1.1137 & 0.1016 & $11219 \mathrm{kV} / \mathrm{m}^{2}$ & 2.54 \\
\hline 1.2661 & 0.1016 & $-16861 \mathrm{kV} / \mathrm{m}^{2}$ & 2.54 \\
\hline 1.4185 & 0.1016 & $8162 \mathrm{kV} / \mathrm{m}^{2}$ & 2.54 \\
\hline 1.5709 & 0.1016 & 0 & 2.54 \\
\hline 1.7233 & 0.1016 & 0. & 2.54 \\
\hline 1.8757 & 0.1016 & 0 . & 2.54 \\
\hline 2.0281 & 0.1016 & 0 . & 2.54 \\
\hline 2.1805 & 0.1016 & 0. & 2.54 \\
\hline 4.0357 & 0.4 & $-0.775 \mathrm{~T} / \mathrm{m}$ & 1.91 \\
\hline 4.7352 & 0.4 & $1.796 \mathrm{~T} / \mathrm{m}$ & 2.54 \\
\hline 5.4387 & 0.4 & $-1.109 \mathrm{~T} / \mathrm{m}$ & 2.54 \\
\hline 7.1617 & 0.4 & $-1.893 \mathrm{~T} / \mathrm{m}$ & 3.18 \\
\hline 7.9237 & 0.717 & $1.975 \mathrm{~T} / \mathrm{m}$ & 3.18 \\
\hline 8.6597 & 0.351 & $-3.368 \mathrm{~T} / \mathrm{m}$ & 1.91 \\
\hline
\end{tabular}

Table 6.5: Lattice properties for the modified scaled HIBALL-II case.

\begin{tabular}{|c|c|c|c|c|c|}
\hline Initial distribution & $\begin{array}{c}\text { Number of } \\
\text { particles }\end{array}$ & Grid size & $\begin{array}{c}\text { Step } \\
\text { size }(\mathrm{cm})\end{array}$ & $\begin{array}{c}\text { Maximum } \\
\text { emittance } \sqrt{\varepsilon_{x} \varepsilon_{y}} \\
(\pi \mathrm{mm} \mathrm{mrad})\end{array}$ & $\begin{array}{c}\text { Spot } \\
\text { area } \\
\left(\mathrm{mm}^{2}\right)\end{array}$ \\
\hline \hline Semi-Gaussian & $\begin{array}{c}40,000 \\
(2 \text { lost })\end{array}$ & $256 \times 256$ & 1. & 4.319 & 1.297 \\
\hline Semi-Gaussian & $\begin{array}{c}80,000 \\
(4 \text { lost })\end{array}$ & $256 \times 256$ & 1. & 4.312 & 1.292 \\
\hline Semi-Gaussian & $\begin{array}{c}10,000 \\
(\text { none lost })\end{array}$ & $128 \times 128$ & 1. & 4.356 & 1.310 \\
\hline K-V & $\begin{array}{c}40,000 \\
(2 \text { lost })\end{array}$ & $256 \times 256$ & 0.5 & 4.334 & 1.295 \\
\hline $\begin{array}{c}40,000 \\
(\text { none lost })\end{array}$ & $256 \times 256$ & 1. & 2.257 & 0.607 \\
\hline
\end{tabular}

Table 6.6: Simulation results for the modified scaled HIBALL-II case. 


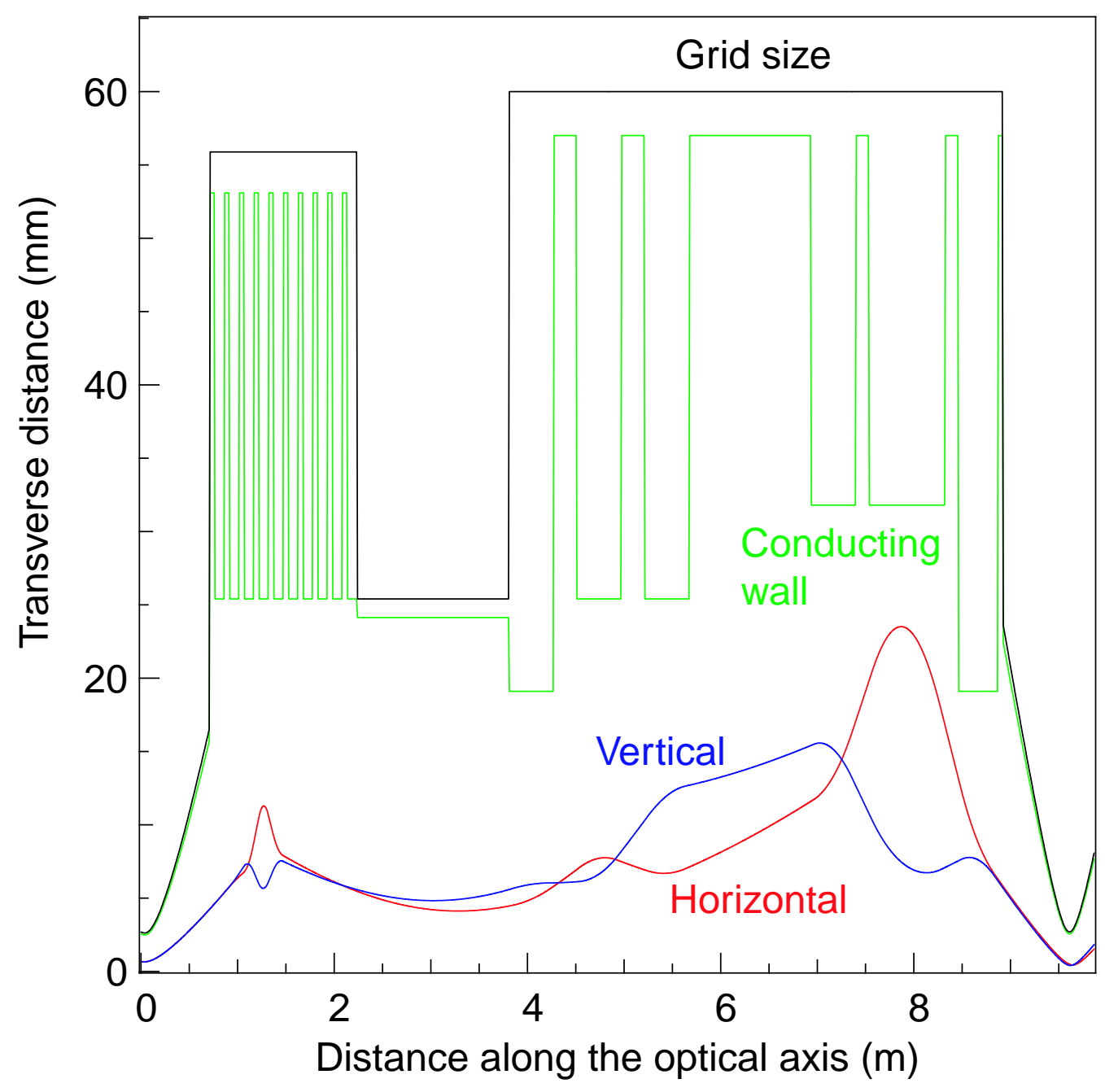

Figure 6.17: The horizontal and vertical beam envelope, extent of the grid, and the location of the conducting boundaries in the simulation of the modified scaled HIBALL-II case. 
increase found in simulations of the Scaled Final Focus Experiment are due to nonlinear space-charge forces that occur during beam expansion.

For the modified scaled HIBALL-II case, no anomalous emittance growth occurred in the experiment [72]. This allows us to make detailed comparisons between the simulation and the experimental results. Phase space comparisons between the experimental measurements and simulations have been described previously [72]. Here, we will only consider the emittance of the beam measured at the diagnostic stations in the experiment, shown in figure 6.18 in addition to the emittance as calculated by WARPxy. This figure shows that there is good qualitative agreement and reasonable quantitative agreement between the experimentally measured emittance and the emittance calculated by WARPxy.

\subsection{The High Scaled Current Case}

For charge neutralization experiments, a beam with a larger current of $400 \mu \mathrm{A}$ was used [72]. In this section, we will describe the beam dynamics up to the neutralization point, in order to understand better how beams behave that have a larger current than the scaled HIBALL-II value. A diagram of the Scaled Final Focus Experiment with the $400 \mu \mathrm{A}$ beam is shown in figure 6.20. Since for this higher current, the beam is closer to the conducting boundaries, we expect image fields to play a larger role than in the scaled HIBALL-II and the modified scaled HIBALL-II case. The beam parameters for this higher scaled current case are given in table 6.7.

Table 6.8 lists the focusing lattice properties, showing the quadrupole strengths

both in the experiment and in the simulation. Possibly due to the beam rotation in the 


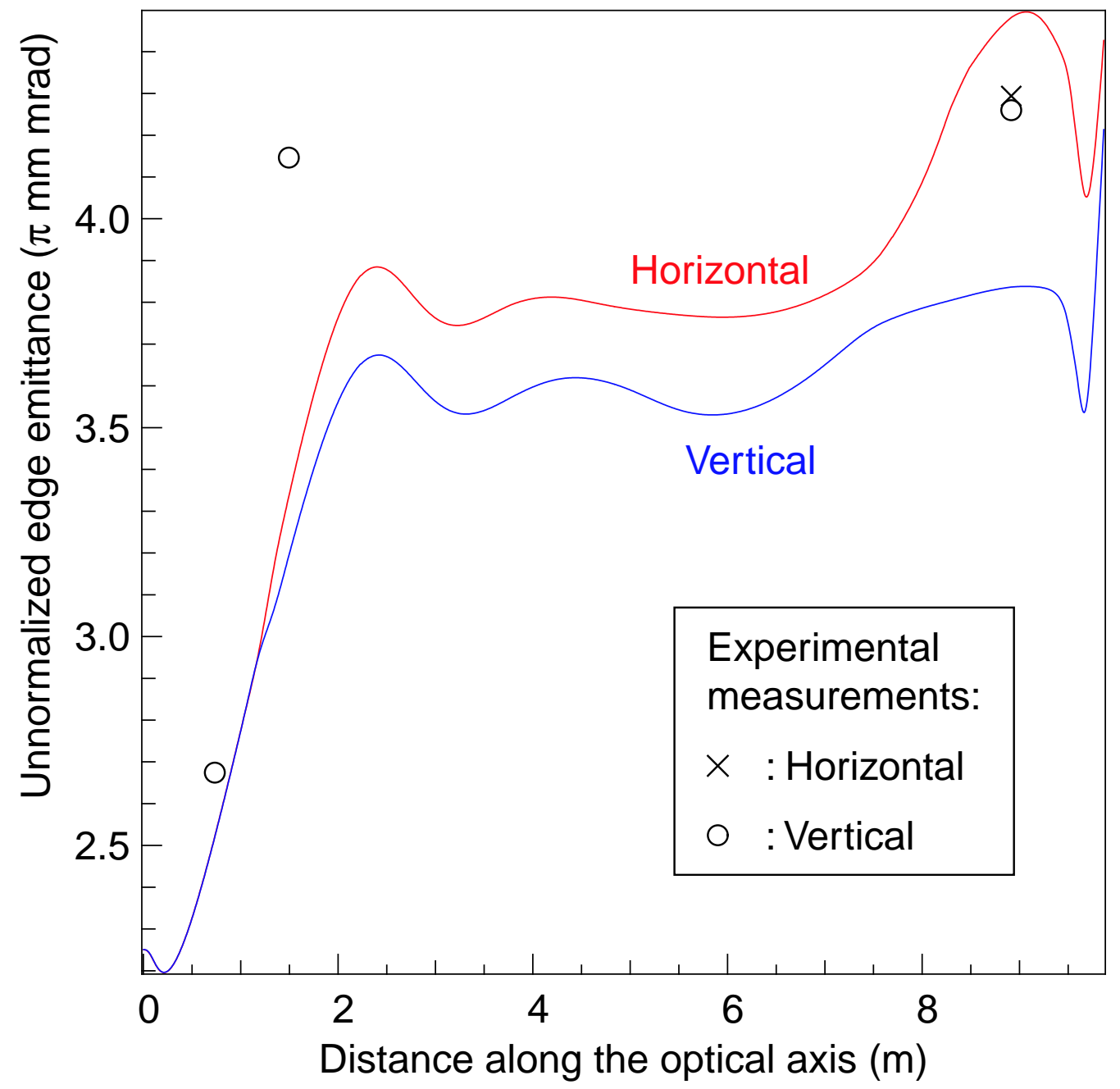

Figure 6.18: The edge emittance of an initially semi-Gaussian beam as a function of the longitudinal distance traveled, as well as the emittance measurements from the experiment, for the modified scaled HIBALL-II case. 


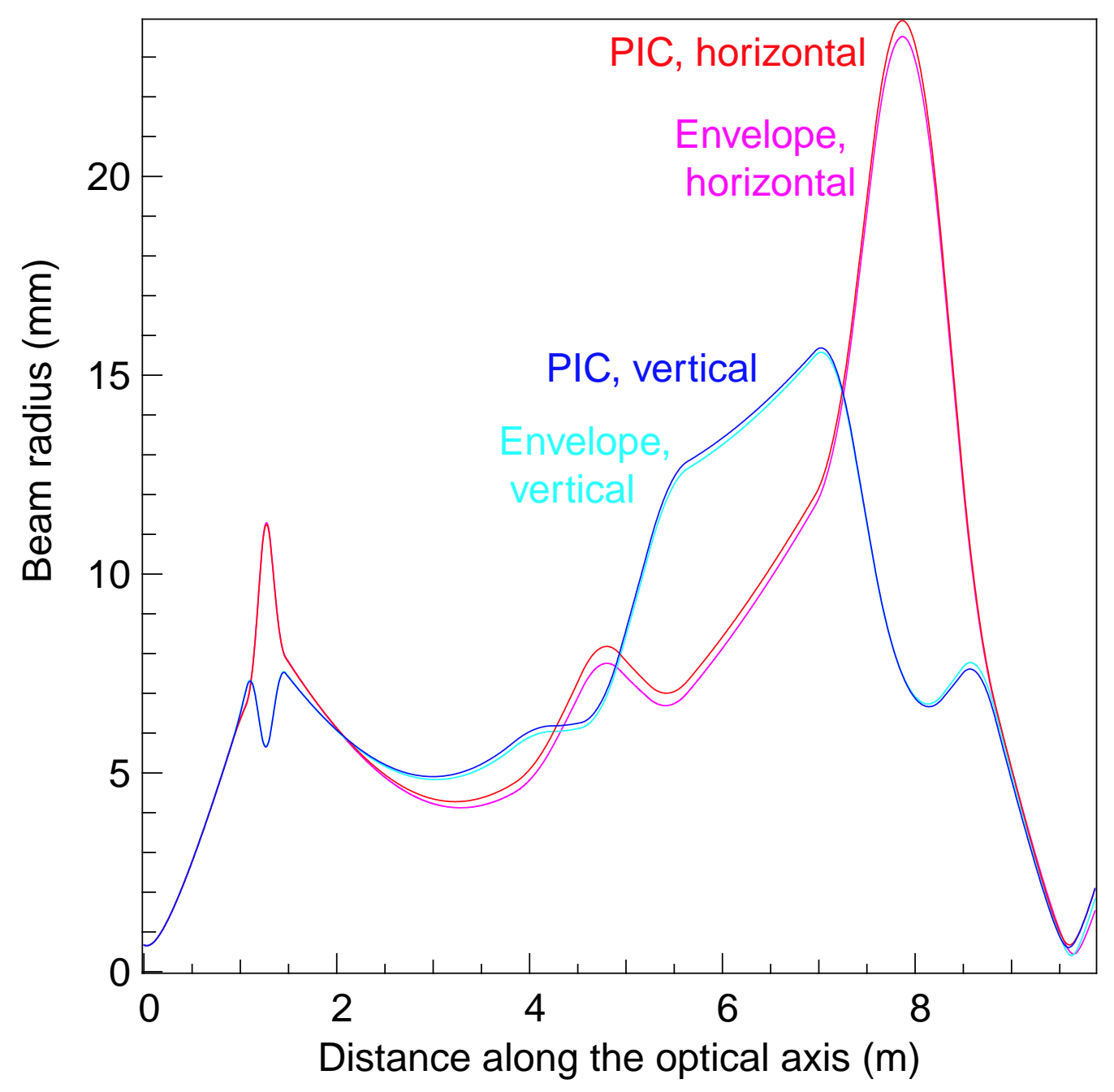

Figure 6.19: The horizontal and vertical beam radius of an initially semi-Gaussian beam as a function of the longitudinal distance traveled, for the modified scaled HIBALL-II case. Both the results from the baseline particle-in-cell simulation and an envelope calculation are shown. 


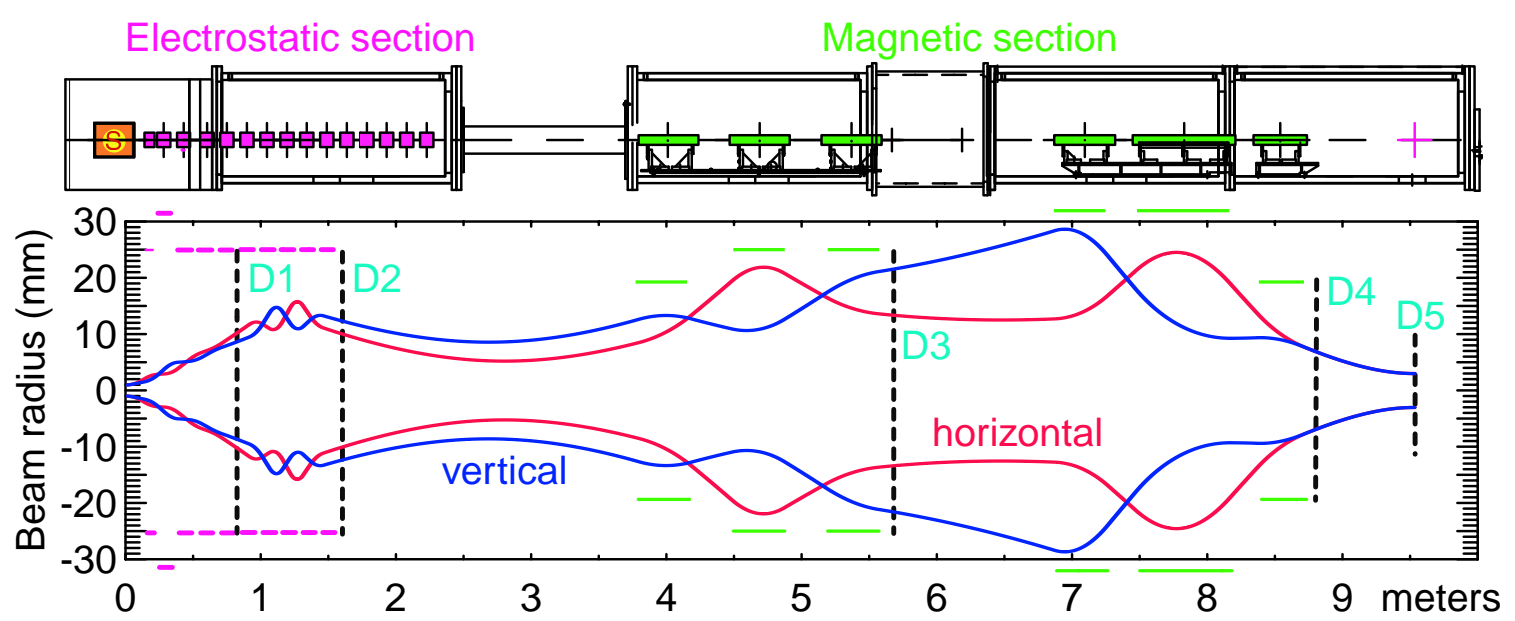

Figure 6.20: The Scaled Final Focus Experiment. The beam envelope shown corresponds to the higher scaled current case.

\begin{tabular}{|l|l|}
\hline Beam energy & $160 \mathrm{keV}$ \\
\hline Initial edge emittance & $5.84 \pi \mathrm{mm} \mathrm{mrad}$ \\
\hline Beam current & $400 \mu \mathrm{A}$ \\
\hline Initial beam parameters & $\begin{array}{l}a_{0}=b_{0}=1.0 \mathrm{~mm} \\
a_{0}^{\prime}=b_{0}^{\prime}=-2.0 \mathrm{mrad}\end{array}$ \\
\hline Final focus spot size (from envelope calculation) & $3.0 \mathrm{~mm}^{2}$ \\
\hline
\end{tabular}

Table 6.7: Beam parameters for the higher scaled current case.

experiment, the beam envelope in the simulation does not accurately reflect the beam envelope in the experiment if exactly the same focusing strengths are used in the simulation as in the experiment. In the simulation, the focusing strengths of some of the quadrupoles were therefore modified slightly in order to stay as close as possible to the experimentally determined beam envelope. The beam radius in the envelope and PIC simulations is shown as a function of the distance along the optical axis in figure 6.21, together with the beam radius measured in the experiment at the diagnostic locations D1 through D4.

The simulations for the high scaled current case started at the injector, assuming a semi-Gaussian distribution initially. The initial emittance in the simulation was chosen such 


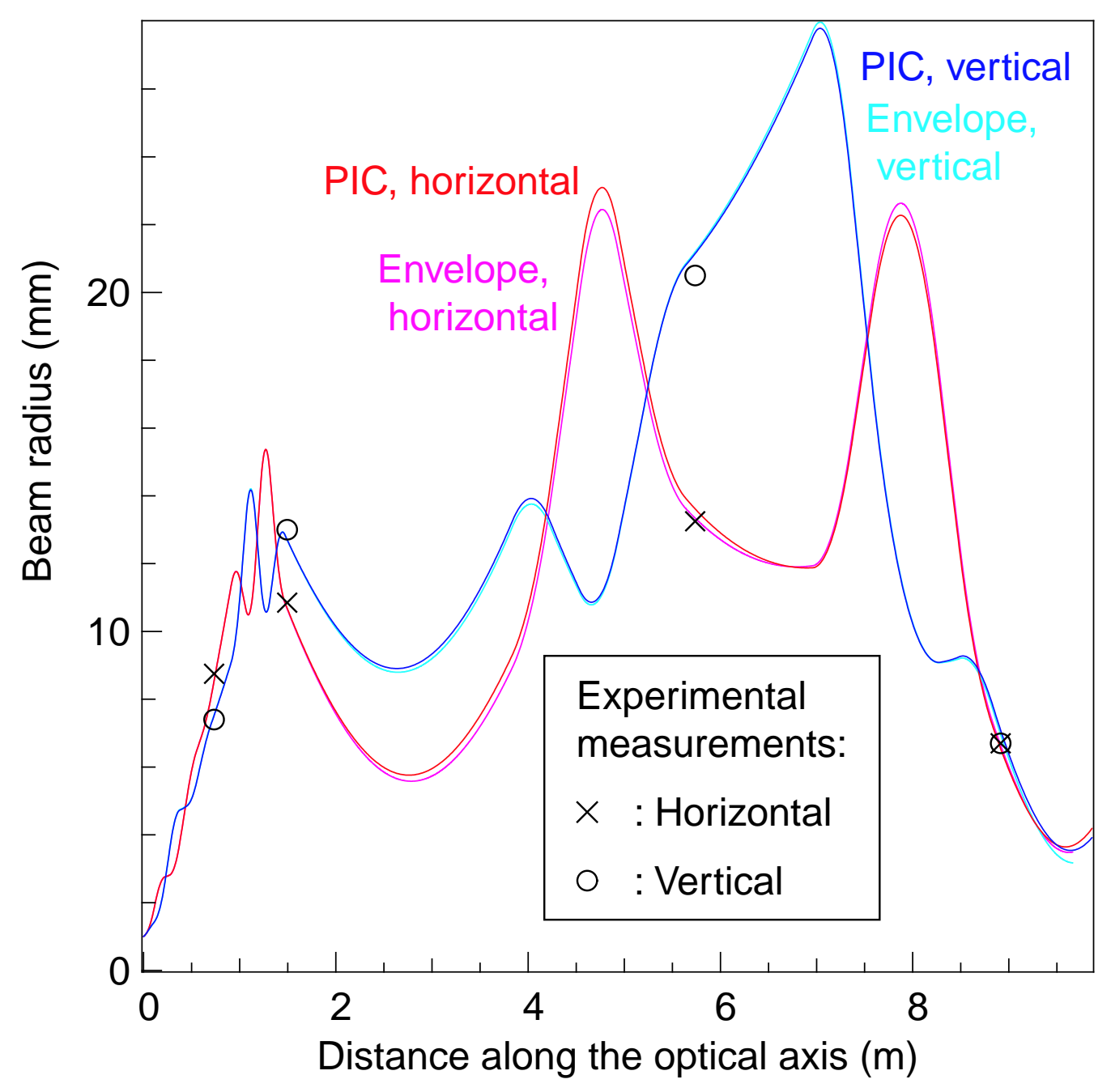

Figure 6.21: The horizontal and vertical beam radius of an initially semi-Gaussian beam as a function of the longitudinal distance traveled for the higher scaled current case. Both the results from the baseline particle-in-cell simulation and an envelope calculation are shown, as well as the beam radius measured at the diagnostic stations D1-D4 in the experiment. 


\begin{tabular}{|c|c|c|c|c|}
\hline $\begin{array}{l}\text { Quadrupole center } \\
\text { locations }(\mathrm{m})\end{array}$ & $\begin{array}{l}\text { Quadrupole } \\
\text { lengths (m) }\end{array}$ & $\begin{array}{c}\text { Field gradient } \\
\text { (experiment) }\end{array}$ & $\begin{array}{l}\text { Field gradient } \\
\text { (simulation) }\end{array}$ & Aperture $(\mathrm{cm})$ \\
\hline 0.0651 & "0.0762 & $15.50 \mathrm{MeV} / \mathrm{m}^{2}$ & 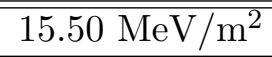 & 2.54 \\
\hline 0.173 & 0.1016 & $-19.51 \mathrm{MeV} / \mathrm{m}^{2}$ & $-19.51 \mathrm{MeV} / \mathrm{m}^{2}$ & 2.54 \\
\hline 0.3254 & 0.1016 & $14.52 \mathrm{MeV} / \mathrm{m}^{2}$ & $14.52 \mathrm{MeV} / \mathrm{m}^{2}$ & 2.54 \\
\hline 0.5041 & 0.1016 & $-5.82 \mathrm{MeV} / \mathrm{m}^{2}$ & $-5.82 \mathrm{MeV} / \mathrm{m}^{2}$ & 2.54 \\
\hline 0.6565 & 0.1016 & $2.48 \mathrm{MeV} / \mathrm{m}^{2}$ & $2.48 \mathrm{MeV} / \mathrm{m}^{2}$ & 2.54 \\
\hline 0.8089 & 0.1016 & 0. & 0. & 2.54 \\
\hline 0.9613 & 0.1016 & $-8.47 \mathrm{MeV} / \mathrm{m}^{2}$ & $-8.47 \mathrm{MeV} / \mathrm{m}^{2}$ & 2.54 \\
\hline 1.1137 & 0.1016 & $16.19 \mathrm{MeV} / \mathrm{m}^{2}$ & $16.19 \mathrm{MeV} / \mathrm{m}^{2}$ & 2.54 \\
\hline 1.2661 & 0.1016 & $-15.98 \mathrm{MeV} / \mathrm{m}^{2}$ & $-15.98 \mathrm{MeV} / \mathrm{m}^{2}$ & 2.54 \\
\hline 1.4185 & 0.1016 & $6.99 \mathrm{MeV} / \mathrm{m}^{2}$ & $6.99 \mathrm{MeV} / \mathrm{m}^{2}$ & 2.54 \\
\hline 1.5709 & 0.1016 & 0 & 0 & 2.54 \\
\hline 1.7233 & 0.1016 & 0. & 0. & 2.54 \\
\hline 1.8757 & 0.1016 & 0. & 0 . & 2.54 \\
\hline 2.0281 & 0.1016 & 0. & 0. & 2.54 \\
\hline 2.1805 & 0.1016 & 0. & 0. & 2.54 \\
\hline 4.0357 & 0.4 & $-1.86 \mathrm{~T} / \mathrm{m}$ & $-1.86 \mathrm{~T} / \mathrm{m}$ & 1.91 \\
\hline 4.7352 & 0.4 & $2.78 \mathrm{~T} / \mathrm{m}$ & $2.78 \mathrm{~T} / \mathrm{m}$ & 2.54 \\
\hline 5.4387 & 0.4 & $-1.08 \mathrm{~T} / \mathrm{m}$ & $-1.20 \mathrm{~T} / \mathrm{m}$ & 2.54 \\
\hline 7.1617 & 0.4 & $-2.21 \mathrm{~T} / \mathrm{m}$ & $-2.12 \mathrm{~T} / \mathrm{m}$ & 3.18 \\
\hline 7.9237 & 0.717 & $2.00 \mathrm{~T} / \mathrm{m}$ & $1.99 \mathrm{~T} / \mathrm{m}$ & 3.18 \\
\hline 8.6597 & 0.351 & $-2.62 \mathrm{~T} / \mathrm{m}$ & $-2.84 \mathrm{~T} / \mathrm{m}$ & 1.91 \\
\hline
\end{tabular}

Table 6.8: Lattice properties for the higher scaled current case. 
that the emittance measured at D1 in the experiment agrees with the emittance calculated at D1 in the simulation. In earlier simulations, the initial emittance was set equal to the measured emittance at D1, but this resulted in poorer agreement between the simulation and the experimental results [25]. As in the simulations of the modified scaled HIBALL-II case, the presence of the conducting boundaries of the first five electrostatic quadrupoles was not included. They were replaced by circular conducting boundaries at a distance of about four times the beam radius. The conducting boundaries of the other electrostatic and magnetic quadrupoles were included though. As the beam converges onto the spot, the grid size is scaled down to four times the beam radius at each time step. Figure 6.22 shows the beam envelope, grid size, and location of the conducting boundaries in this simulation.

The base line case used 40,000 particles on a $256 \times 256$ grid with spatial filtering and fourfold symmetry with a step size of $1 \mathrm{~cm}$. In order to guarantee convergence, several additional simulations were done using a different number of particles, grid size, or step size. A simulation in which chromatic aberrations corresponding to a source temperature of $1100^{\circ} \mathrm{C}$ were included, did not produce noticeably different results. Chromatic aberrations were therefore subsequently ignored. The simulations and their results are summarized in table 6.9.

Figure 6.23 shows the emittance calculated from the PIC simulation, as well as the emittance measurements available from the experiment. The emittance near the focal spot is about $10.2 \pi \mathrm{mm} \mathrm{mrad}$, compared to $5.84 \pi \mathrm{mm} \mathrm{mrad}$ initially. This leads to a $16.8 \%$ increase in the focal spot area compared with the K-V envelope calculation. The emittance calculated from the simulation agrees reasonably well with the measured emittance. 


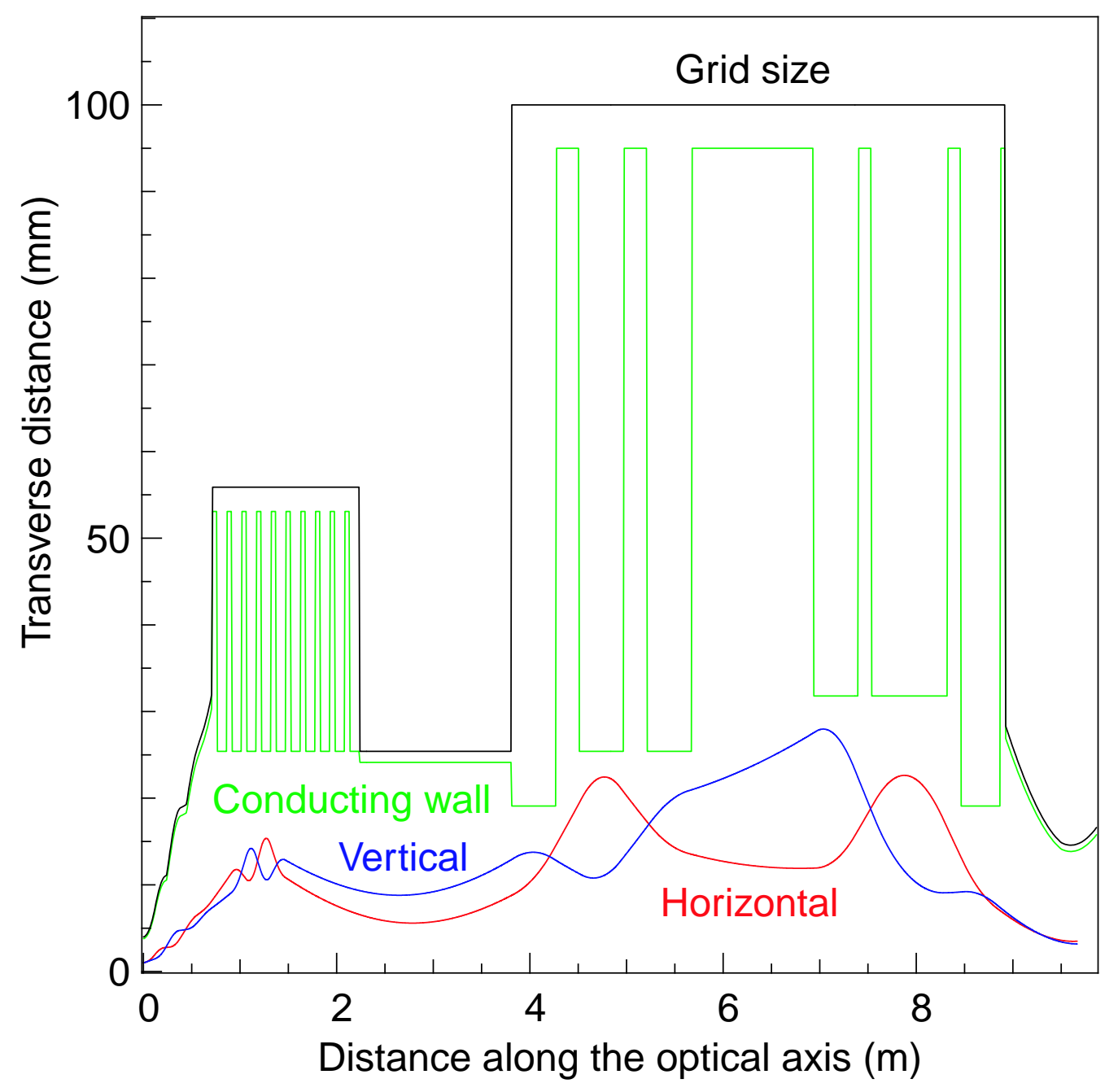

Figure 6.22: The horizontal and vertical beam envelope, extent of the grid, and the location of the conducting boundaries in the simulation of the higher scaled current case. 


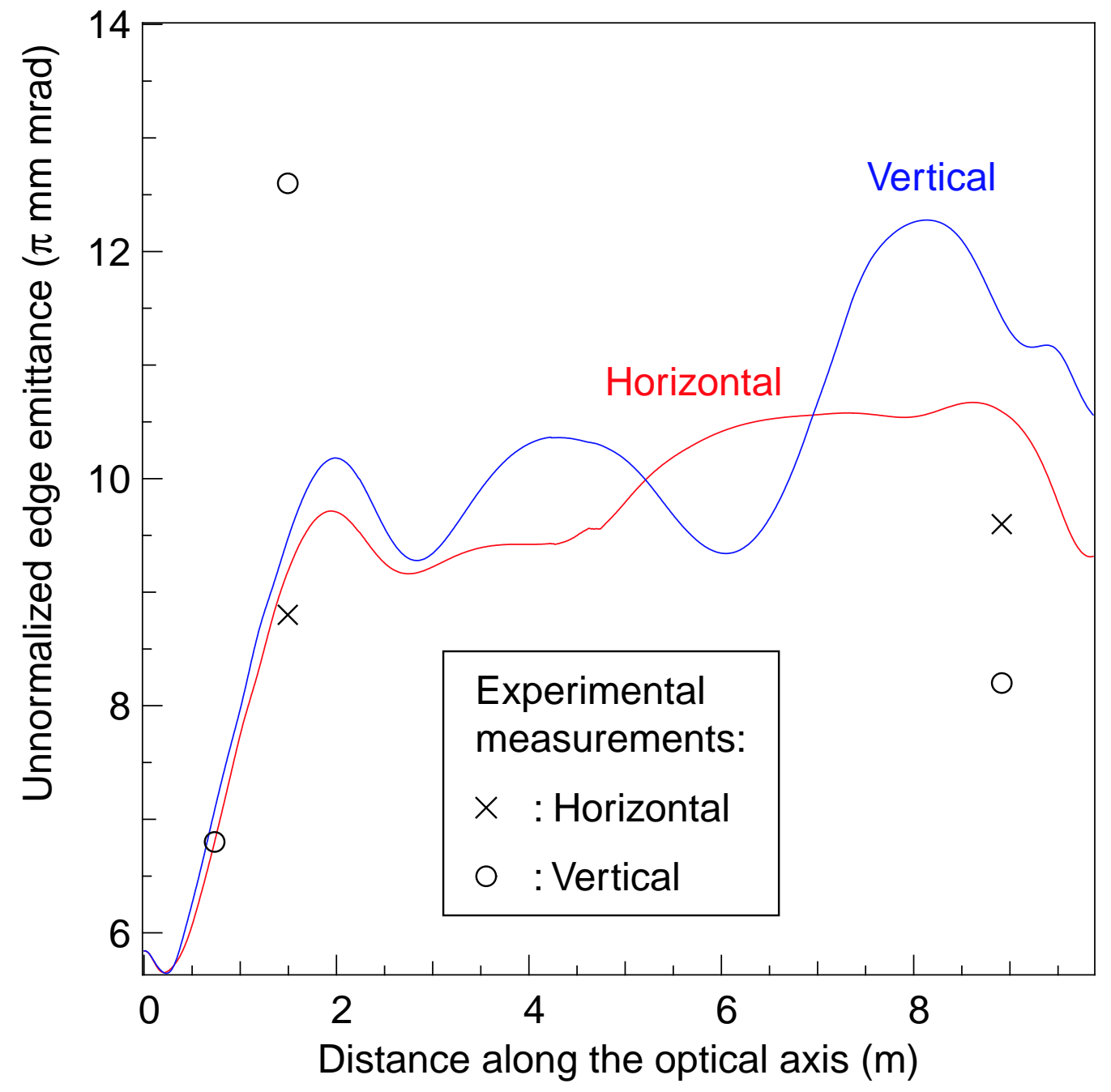

Figure 6.23: The edge emittance of an initially semi-Gaussian beam as a function of the longitudinal distance traveled, as well as the emittance measurements from the experiment, for the higher scaled current case. 


\begin{tabular}{|c|c|c|c|c|c|}
\hline Initial distribution & $\begin{array}{c}\text { Number of } \\
\text { particles }\end{array}$ & Grid size & $\begin{array}{c}\text { Step } \\
\text { size }(\mathrm{cm})\end{array}$ & $\begin{array}{c}\text { Maximum } \\
\text { emittance } \sqrt{\varepsilon_{x} \varepsilon_{y}} \\
(\pi \mathrm{mm} \text { mrad })\end{array}$ & $\begin{array}{c}\text { Spot } \\
\text { area } \\
\left(\mathrm{mm}^{2}\right)\end{array}$ \\
\hline \hline Semi-Gaussian & $\begin{array}{c}40,000 \\
(106 \text { lost })\end{array}$ & $256 \times 256$ & 1. & 11.4 & 40.7 \\
\hline Semi-Gaussian & $\begin{array}{c}80,000 \\
(206 \text { lost })\end{array}$ & $256 \times 256$ & 1. & 11.4 & 40.6 \\
\hline Semi-Gaussian & $\begin{array}{c}10,000 \\
(23 \text { lost })\end{array}$ & $128 \times 128$ & 1. & 11.0 & 40.9 \\
\hline K-V & $\begin{array}{c}40,000 \\
(108 \text { lost })\end{array}$ & $256 \times 256$ & 0.5 & 11.4 & 40.1 \\
\hline $\begin{array}{c}40,000 \\
(\text { none lost })\end{array}$ & $256 \times 256$ & 1. & 5.92 \\
\hline
\end{tabular}

Table 6.9: Simulation results for the higher scaled current case.

The emittance increase and subsequent increase in the spot size is mostly due to the nonlinear space-charge field in the beam, causing severe distortions in phase space that are in principle reversible. Figure 6.24 shows the emittance behavior of a $\mathrm{K}-\mathrm{V}$ beam, which is characterized by a linear space-charge field. Virtually no increase in emittance is observed, and the focal spot size is only $0.36 \%$ larger than in the envelope calculation.

Replacing the conducting boundaries in the simulation by a single circular conducting boundary with a large radius produces almost no changes in the simulation results. The nonlinear image charges do not significantly affect the beam.

Phase space measurements were made in the simulation at the same diagnostic locations as in the experiment for ease of comparison. As the beam propagates through the final focus system, rather severe distortions occur in its phase space. Figures 6.25 through 6.31 show the sheared horizontal and vertical phase space at the diagnostic locations D1 through D4, showing both the experimental data and the simulation results. Measurements of the horizontal phase space at D1 were not available from the experiment. The beam 


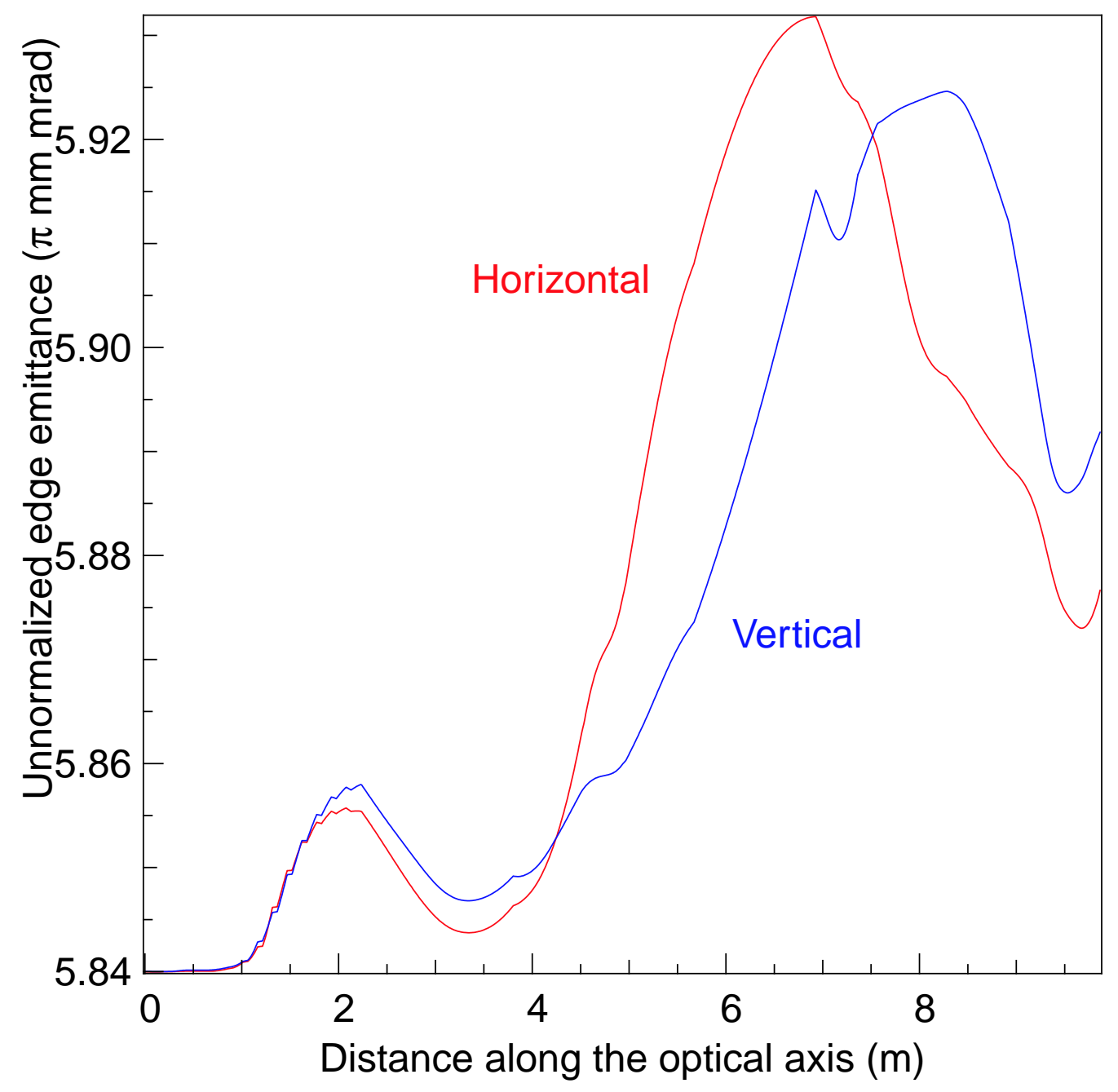

Figure 6.24: The edge emittance of an initially K-V beam as a function of the longitudinal distance traveled, for the higher scaled current case. 
undergoes distortions from an s-shape to a z-shape and finally back to an s-shape. Figures 6.25 through 6.25 show reasonably good qualitative agreement in the vertical phase space plots between the simulation and the experimental results. The shape of the phase space distortions that occur in the experiment are replicated in the simulation, although the simulation and experimental results do not always agree on the severity of the distortions. For the horizontal phase space, it is more difficult to compare the simulation and experimental results due to severe distortions in the experimental measurements caused by additional imperfections such as misalignments.

Several explanations can be given why the agreement between the simulation and the experimental results is not perfect. The simulations represent an idealized system, and do not take into account any multipole fields. Although the beam is compressing longitudinally in the experiment, no 3D effects were included in the simulation. Finally, the semi-Gaussian distribution used in the simulation is probably only a crude approximation to the actual initial beam distribution, which is unknown. But one of the major causes of the disparities is probably the beam rotation that was found in the experiment. Since the simulation was set up such that the beam envelope agrees with experimentally measured beam radii at the diagnostic locations (figure 6.21), the envelopes may disagree elsewhere due to the rotation of the beam in the experiment. 

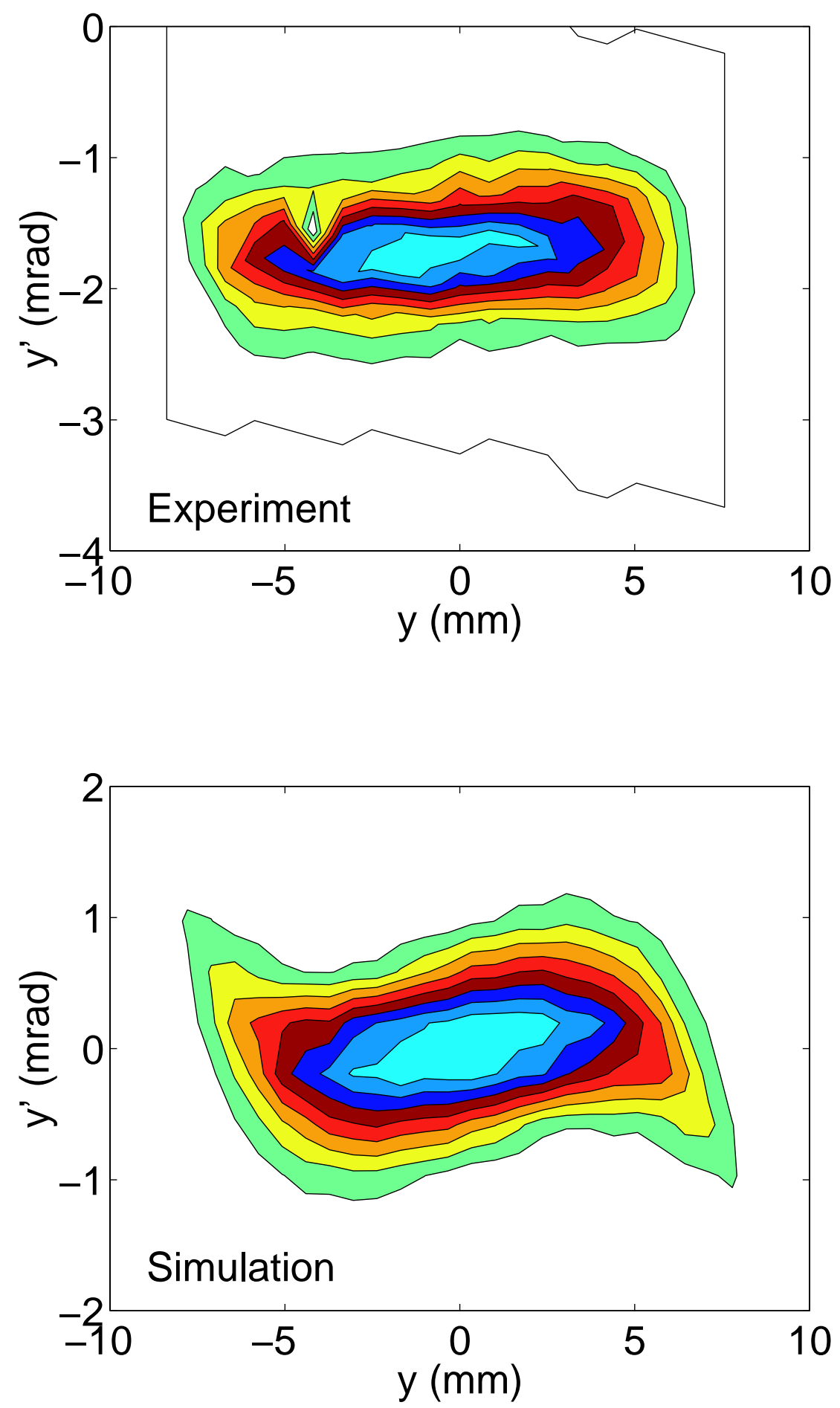

Figure 6.25: Sheared vertical phase space at diagnostic station D1. The density levels shown in this figure are evenly spaced. 

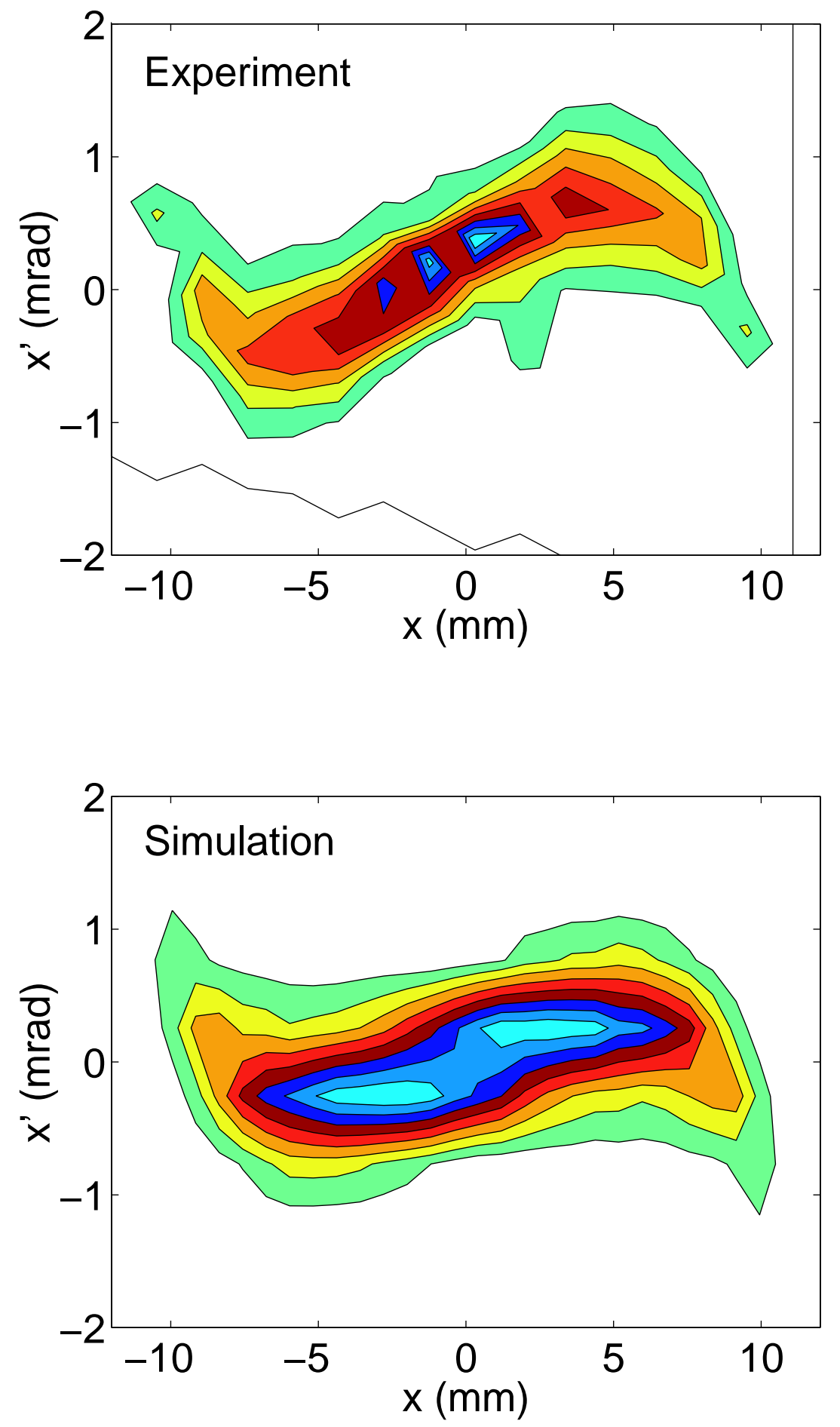

Figure 6.26: Sheared horizontal phase space at diagnostic station D2. The density levels shown in this figure are evenly spaced. 

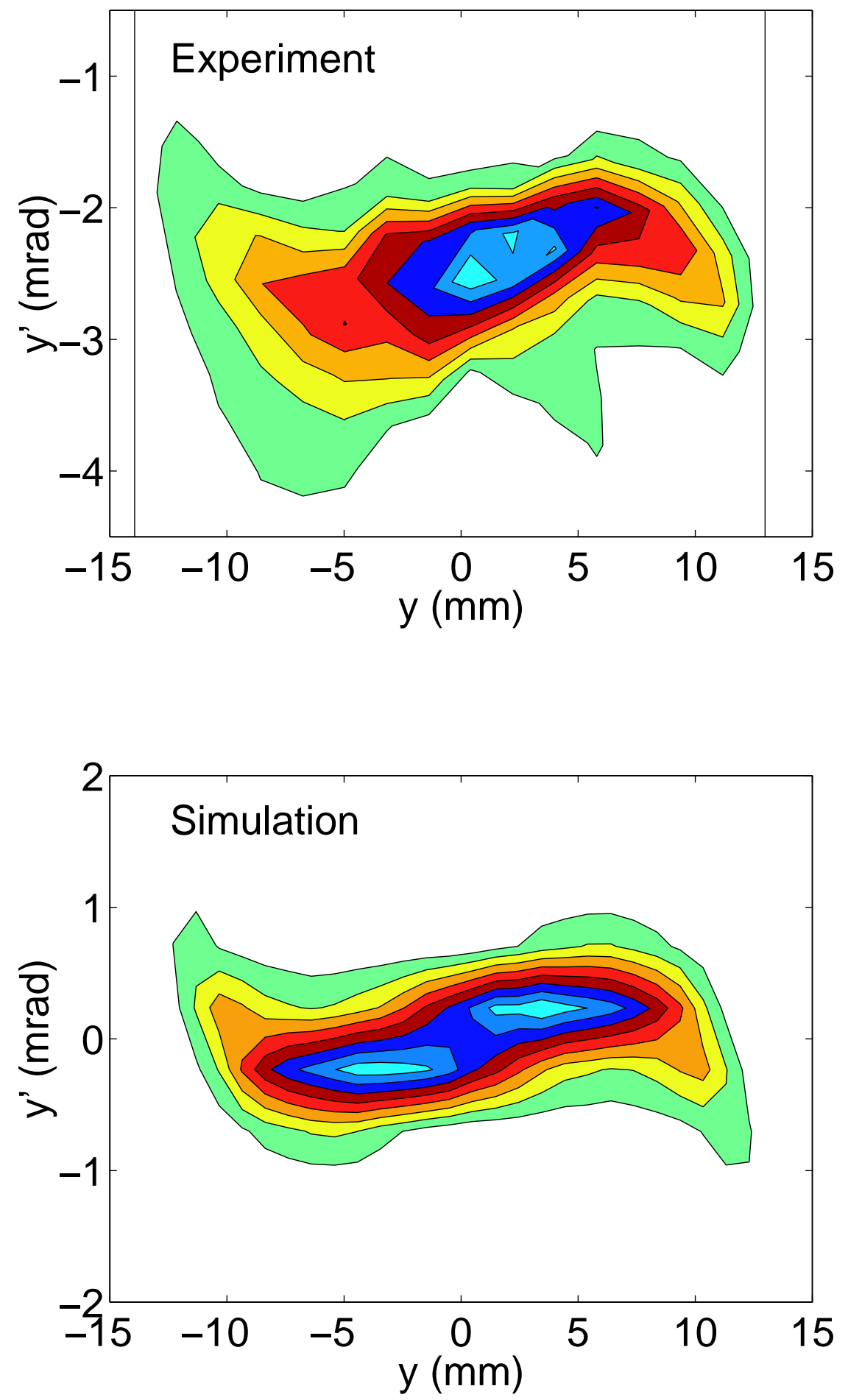

Figure 6.27: Sheared vertical phase space at diagnostic station D2. The density levels shown in this figure are evenly spaced. 

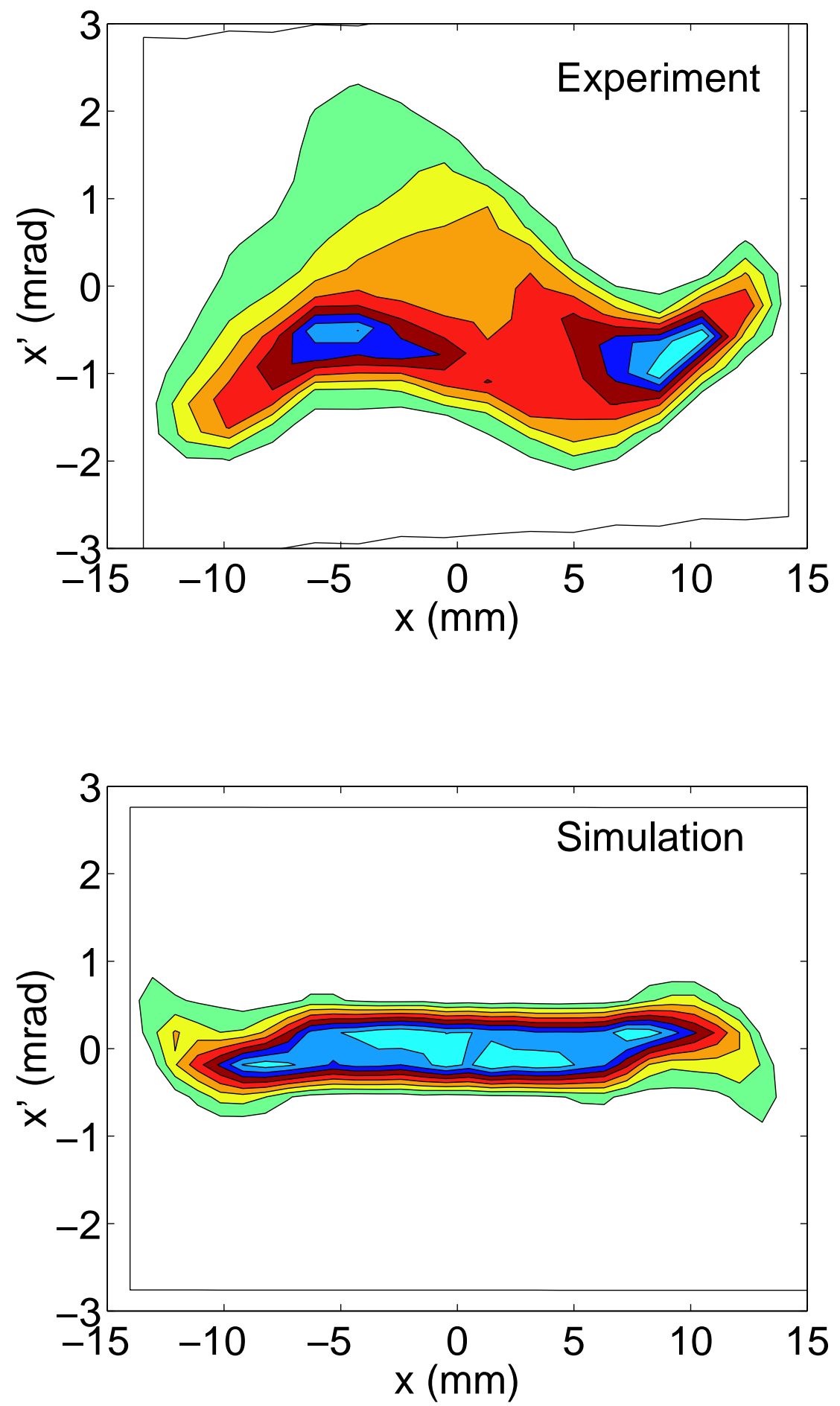

Figure 6.28: Sheared horizontal phase space at diagnostic station D3. The density levels shown in this figure are evenly spaced. 

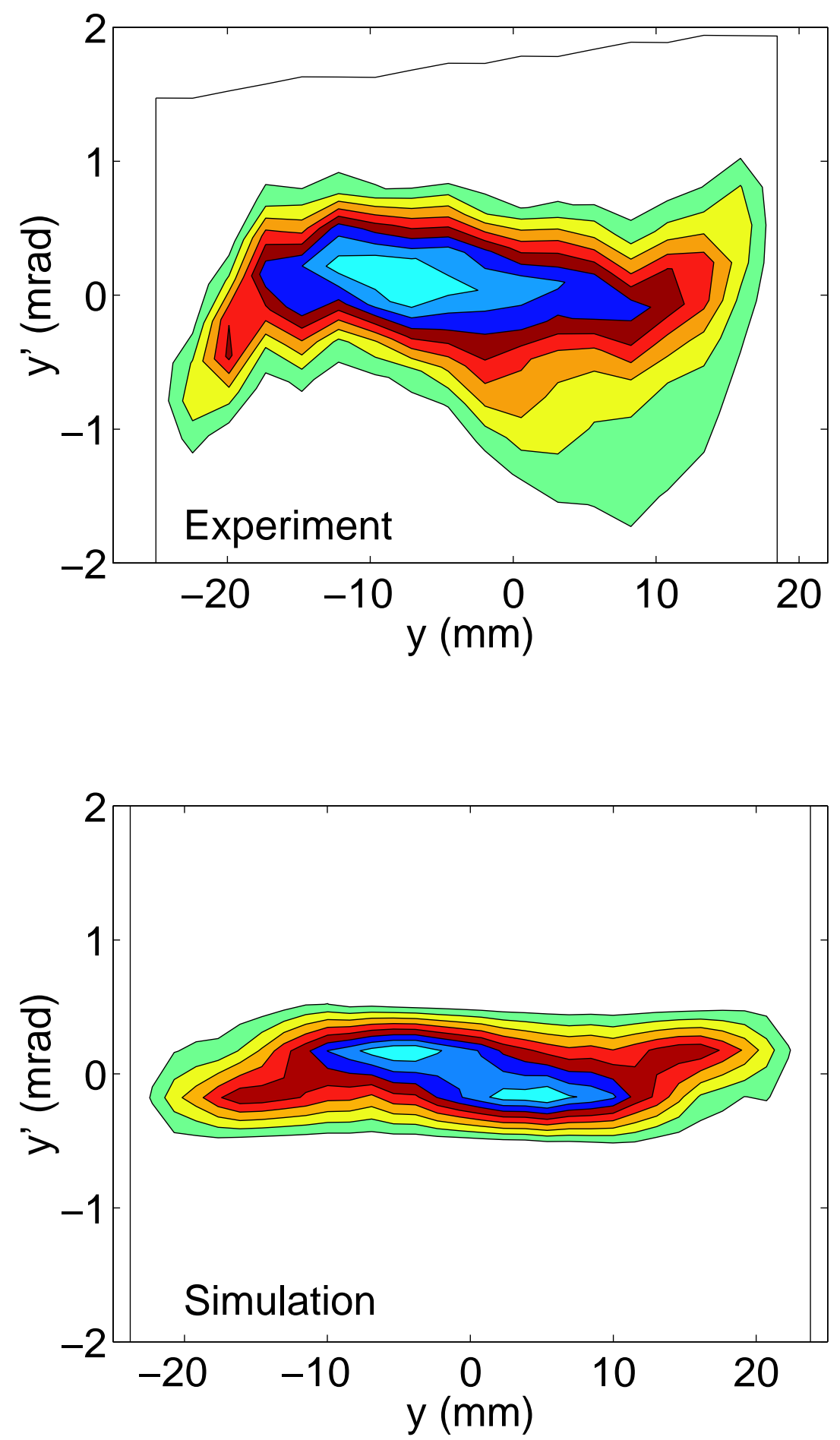

Figure 6.29: Sheared vertical phase space at diagnostic station D3. The density levels shown in this figure are evenly spaced. 

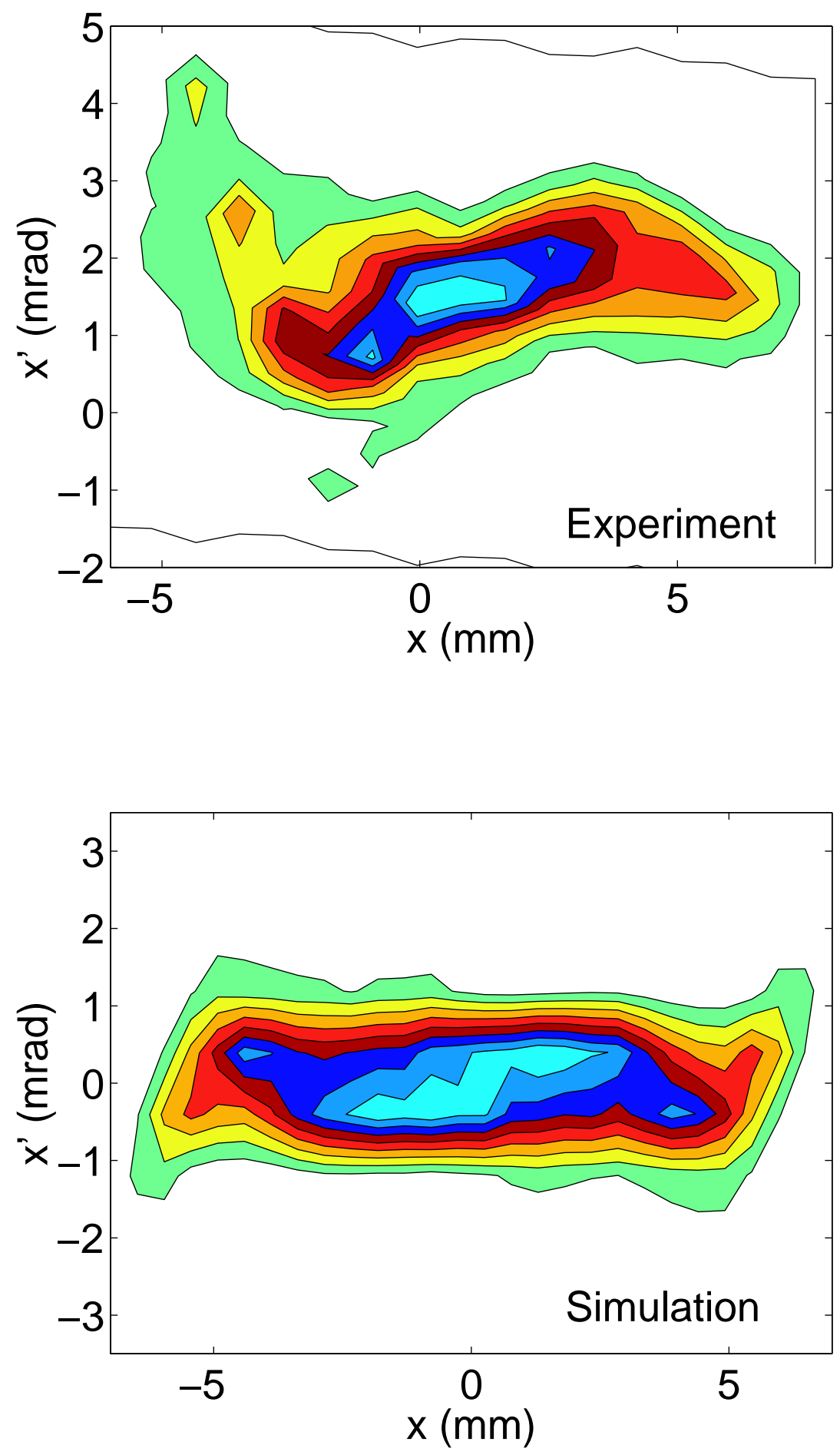

Figure 6.30: Sheared horizontal phase space at diagnostic station D4. The density levels shown in this figure are evenly spaced. 

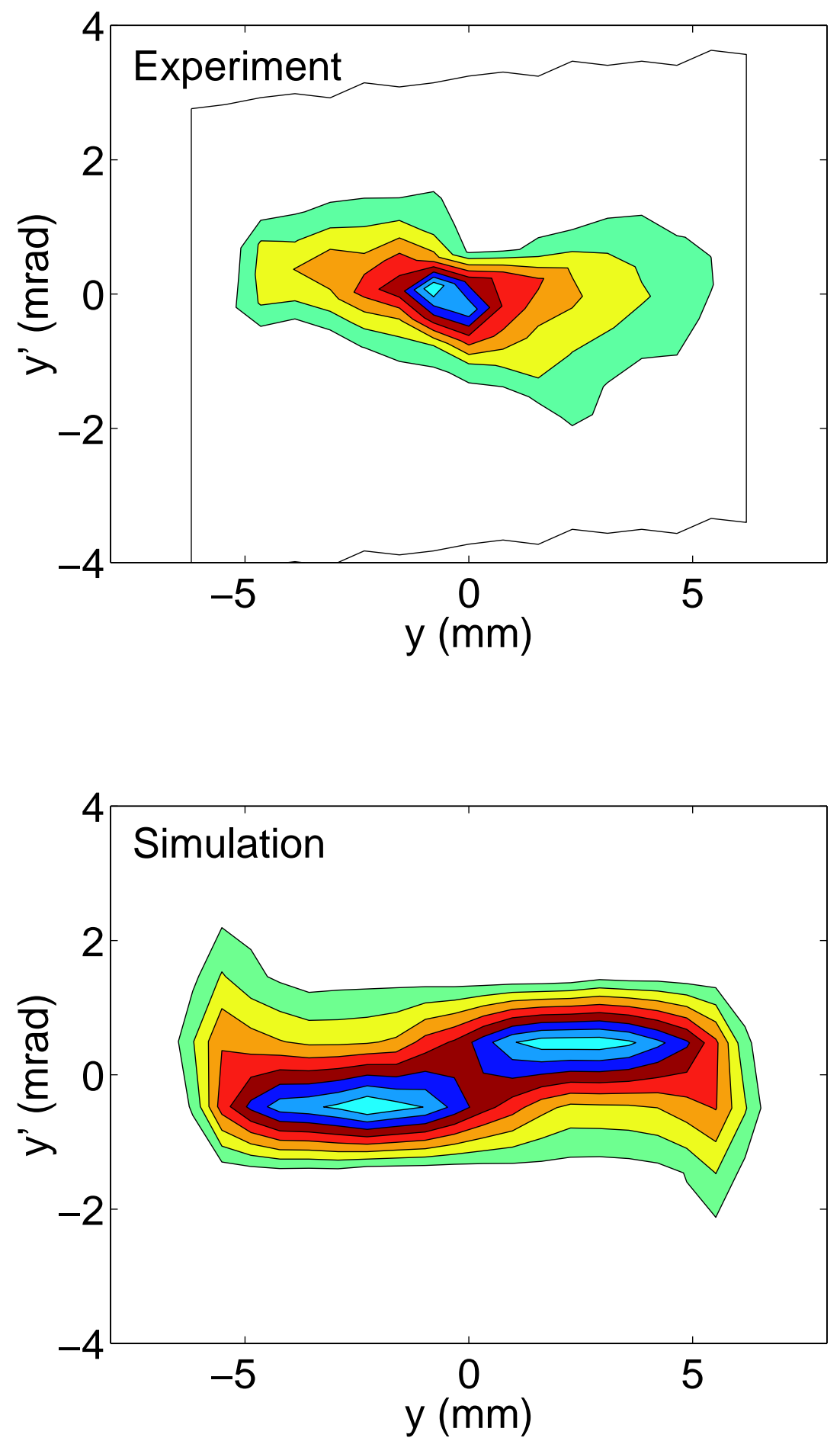

Figure 6.31: Sheared vertical phase space at diagnostic station D4. The density levels shown in this figure are evenly spaced. 


\subsection{Beam Rotation}

In the simulations done so far, we have not included the effects of beam rotation.

In the experiment however, the beam has been observed to rotate. In this section, we will give a possible explanation of the beam rotation and how it can be related to the anomalous emittance growth observed in the scaled HIBALL-II case.

A rotation of the beam can be due to a number of causes, such as the presence of the Earth's magnetic field, an asymmetric effect of image charges for an off-axis beam, or a physical rotation of the focusing quadrupoles or asymmetries in the currents of magnetic quadrupoles, causing the quadrupolar field to be rotated. Whereas the Earth's magnetic field, of the order of $0.05 \mathrm{mT}$ [1], is too weak to significantly affect the beam, simulations have shown that the asymmetric image forces on an off-axis beam are too small to cause the beam to rotate. This leaves one or more quadrupoles being rotated as the most likely cause of the beam rotation observed in the experiment.

A physical rotation of a quadrupole about its longitudinal axis can still be treated by linear theory [8] by describing its field as a superposition of an upright quadrupole and a skew quadrupole. Including the skew quadrupole field will generate cross-terms in the equations of motion (2.35), (2.36). Due to these cross-terms, the emittances in the horizontal and the vertical direction are no longer conserved. Instead, we find two new emittance-like constants of the motion [59]:

$$
\begin{aligned}
\varepsilon_{g}^{2} & =\frac{1}{2}\left(\varepsilon_{x}^{2}+\varepsilon_{y}^{2}\right)+16\left(\Delta x y \Delta x^{\prime} y^{\prime}-\Delta x y^{\prime} \Delta x^{\prime} y\right) \\
\varepsilon_{h}^{2} & =\left\{\varepsilon_{x}^{2} \varepsilon_{y}^{2}\right. \\
& +256\left(\left[\Delta x y \Delta x^{\prime} y^{\prime}\right]^{2}+\left[\Delta x y^{\prime} \Delta x^{\prime} y\right]^{2}\right)
\end{aligned}
$$




$$
\begin{aligned}
& -\quad 256\left(\Delta x^{2} \Delta y^{2}\left[\Delta x^{\prime} y^{\prime}\right]^{2}+\Delta x^{\prime 2} \Delta y^{\prime 2}[\Delta x y]^{2}\right) \\
& -\quad 256\left(\Delta x^{2} \Delta y^{\prime 2}\left[\Delta x^{\prime} y\right]^{2}+\Delta x^{\prime 2} \Delta y^{2}\left[\Delta x y^{\prime}\right]^{2}\right) \\
& +512\left(\Delta x x^{\prime} \Delta y^{\prime 2} \Delta x y \Delta x^{\prime} y+\Delta x^{\prime 2} \Delta y y^{\prime} \Delta x y \Delta x y^{\prime}\right) \\
& +512\left(\Delta x^{2} \Delta y y^{\prime} \Delta x^{\prime} y \Delta x^{\prime} y^{\prime}+\Delta x x^{\prime} \Delta y^{2} \Delta x^{\prime} y^{\prime} \Delta x^{\prime}\right) \\
& \left.-\quad 512\left(\Delta x y \Delta x y^{\prime}+\Delta x x^{\prime} \Delta y y^{\prime} \Delta x y \Delta x^{\prime} y^{\prime}+\Delta x x^{\prime} \Delta y y^{\prime} \Delta x y^{\prime} \Delta x^{\prime} y\right)\right\}^{\frac{1}{2}}
\end{aligned}
$$

in which the operator $\Delta$ is defined by

$$
\Delta u v \equiv\langle u v\rangle-\langle u\rangle\langle v\rangle
$$

Note that in the absence of cross-correlations between $x$ and $y$, these expressions reduce to the arithmetic and geometric mean of $\varepsilon_{x}^{2}$ and $\varepsilon_{y}^{2}$, which are two independent quantities [59]. In the absence of nonlinear fields, these generalized emittances are conserved in the presence of beam and quadrupole rotations, instead of $\varepsilon_{x}^{2}$ and $\varepsilon_{y}^{2}$.

In the experiment, the horizontal and vertical emittance $\varepsilon_{x}^{2}$ and $\varepsilon_{y}^{2}$ are measured without taking any beam rotation into consideration. These second-order moments of the beam may increase substantially due to a rotation of the beam, although the true $4 \mathrm{D}$ phase space volume is conserved. By only measuring the horizontal and vertical emittance of a rotated beam, we are looking at a particular projection of phase space which is not indicative of the true phase space occupied by the beam. This may result in huge increases in the measured horizontal and vertical emittance, although the 4D phase space volume occupied by the beam may be conserved.

A set of ten first-order differential equations may be derived for the evolution of the second-order moments of the distribution, taking the beam rotation and also misalignments 
into consideration. These ten equations replace the four first-order differential equations for $a, a^{\prime}, b, b^{\prime}$ for a non-rotating, on-axis beam. By solving this set of equations numerically, the linear behavior of the beam in the presence of rotated quadrupoles can be calculated quickly [8]. In this way, we can find the rotation angle of the beam, as well as the change in the horizontal and vertical emittance of the beam due to the beam rotation. These results can then be compared to particle-in-cell simulations of a beam in a system with rotated quadrupoles.

Whereas the generalized emittance is constant in the linear system, in a particlein-cell simulation it will increase due to the presence of nonlinearities, such as space-charge and geometric aberrations. This is similar to the increase in the horizontal and vertical emittance calculated in a particle-in-cell simulation of a nonrotating beam, compared with the constant horizontal and vertical emittance in an envelope calculation. For most of the cases we have looked at, the spurious increase in the horizontal and vertical emittance due to beam rotation is much larger than the increase due to nonlinearities. Therefore, the results from the linear analysis agree fairly well with the results from particle-in-cell simulations.

Since the beam rotation is first observed at diagnostic station D3, we expect it to be caused by a rotation of one or more of the first three magnetic quadrupoles. A rotation of any of these quadrupoles will cause the beam to rotate, which will lead to an increase in the horizontal and vertical emittance. For a given rotation of these quadrupoles, we can calculate the horizontal and vertical emittance as a function of the longitudinal distance by integrating the ten moment equations described above. The rotation angle can be found by matching the emittance increase found in the calculation to the emittance measured 


\begin{tabular}{|c|l|}
\hline Diagnostic station & Emittance $(\pi \mathrm{mm} \mathrm{mrad})$ \\
\hline \hline $\mathrm{D} 2$ & 1.58 (vertical) \\
& 8.79 (horizontal) \\
$\mathrm{D} 4$ & 9.89 (vertical) \\
& $9.32\left(\sqrt{\varepsilon_{x} \varepsilon_{y}}\right)$ \\
\hline
\end{tabular}

Table 6.10: Emittance measured in the experiment at the second and fourth diagnostic station in the scaled HIBALL-II case.

in the experiment. Since there are three quadrupoles that may be rotated, for each case there exist more than one combination of quadrupole rotations that can produce a given emittance increase. By looking at the emittance increase and beam rotation in different cases, we can determine which combination of quadrupole rotations is consistent with all measurements.

The beam rotation for an elliptical beam is defined as the smallest angle the beam has to be rotated by for it to be upright. For a uniform beam, this angle can be calculated from

$$
\phi=\frac{1}{2} \arcsin \left(\frac{2 \Delta x y}{\sqrt{\left(\Delta x^{2}-\Delta y^{2}\right)^{2}+4(\Delta x y)^{2}}}\right) \operatorname{sign}\left(\Delta x^{2}-\Delta y^{2}\right),
$$

in which $\Delta$ is again defined by equation (6.22). This equation was used to determine the beam rotation angle in the experiment and in the simulations.

In the scaled HIBALL-II case, the emittance increases by a factor of six between the diagnostic stations D2 and D4 in the experiment [70]. Table 6.10 lists the emittance measurements at the second and fourth diagnostic station in the experiment.

Solving the system of ten first-order differential equations for different combinations of rotation angles for the first three magnetic quadrupoles revealed that quite small rotation angles may produce the sixfold emittance increase found in the experiment. Table 6.11 gives some examples of rotation angles producing the emittance increase observed 


\begin{tabular}{|c|c|c|c|c|c|c|}
\hline Case & $\begin{array}{c}\text { First } \\
\text { magnet }\end{array}$ & $\begin{array}{c}\text { Second } \\
\text { magnet }\end{array}$ & $\begin{array}{c}\text { Third } \\
\text { magnet }\end{array}$ & $\begin{array}{c}\text { Fourth } \\
\text { magnet }\end{array}$ & $\begin{array}{c}\text { Fifth } \\
\text { magnet }\end{array}$ & $\begin{array}{c}\text { Sixth } \\
\text { magnet }\end{array}$ \\
\hline \hline$(\mathrm{a})$ & $5.7^{\circ}$ & $0^{\circ}$ & $0^{\circ}$ & $0^{\circ}$ & $0^{\circ}$ & $0^{\circ}$ \\
\hline (b) & $0^{\circ}$ & $1.24^{\circ}$ & $0^{\circ}$ & $0^{\circ}$ & $0^{\circ}$ & $0^{\circ}$ \\
\hline$(\mathrm{c})$ & $0^{\circ}$ & $0^{\circ}$ & $1.55^{\circ}$ & $0^{\circ}$ & $0^{\circ}$ & $0^{\circ}$ \\
\hline$(\mathrm{d})$ & $0.67^{\circ}$ & $-0.67^{\circ}$ & $0.67^{\circ}$ & $0^{\circ}$ & $0^{\circ}$ & $0^{\circ}$ \\
\hline$(\mathrm{e})$ & $0.62^{\circ}$ & $-0.62^{\circ}$ & $0.62^{\circ}$ & $0.62^{\circ}$ & $0.62^{\circ}$ & $0.62^{\circ}$ \\
\hline
\end{tabular}

Table 6.11: Several combinations of rotation angles for the magnetic quadrupoles that give rise to a sixfold increase in the horizontal and vertical emittance. These results were found by integrating the linear moment equations.

in the experiment. Since the wiring of the second magnetic quadrupole is opposite from the other magnets [71], we may expect the second magnet to be rotated in the opposite direction (cases (d) and (e)). Rotating the first three magnets in the same direction by the same degree did not reproduce the emittance measurements.

Once we determined the rotation angles of the magnets that give the observed emittance increase, we can run a transverse slice PIC simulation using the rotation angles found to check the results of the linear theory. This was done for the second example in table 6.11 , in which the first magnetic quadrupole was rotated by $1.24^{\circ}$. Just as in the baseline case for the previous scaled HIBALL-II beam simulations, a step size of $1 \mathrm{~cm}$ was used. The grid size was increased to $256 \times 256$, using 160,000 particles, since unlike the baseline case, fourfold symmetry could not be used in this simulation due to the presence of the rotated quadrupole. The lattice parameters given in table 6.2 were used for this simulation, adding a $1.24^{\circ}$ rotation to the second magnetic quadrupole.

Figure 6.32 shows the horizontal and vertical beam radius in the presence of a $1.24^{\circ}$ rotation of the second magnetic quadrupole, as calculated from the moment equations and by doing a WARPxy simulation. Comparison with the beam envelope in the absence of 
any magnet rotations, also shown in figure 6.32 , reveals that the beam radii do not change significantly due to the magnet rotation.

Even though the quadrupole rotation angle is small, the subsequent beam rotation angle is large. Figure 6.33 shows the beam orientation at several locations along the lattice, as determined from the moment locations. This figure shows that the beam undergoes a significant rotation due to one quadrupole being rotated by $1.24^{\circ}$.

Figure 6.35 shows the emittance as calculated by the WARPxy simulations, using a $\mathrm{K}-\mathrm{V}$ and semi-Gaussian beam distribution initially, and the emittance as calculated by the moment equations. The emittance increases sharply upon encountering the rotated quadrupole, and decreases thereafter. The difference between the moment calculation and the WARPxy simulation is small for the $\mathrm{K}-\mathrm{V}$ beam; for the initially semi-Gaussian beam, the emittance increase is somewhat larger due to the presence of nonlinear space-charge forces. This effect is more pronounced if we look at the generalized emittances $\varepsilon_{g}$ and $\varepsilon_{h}$, shown in figure 6.36. Whereas the generalized emittances are conserved for the $\mathrm{K}-\mathrm{V}$ beam, they increase for a semi-Gaussian beam.

To investigate the cause of the emittance growth and the beam rotation, a series of experiments was done using different focusing solutions [81]. This allowed us to measure the beam rotation if some of the quadrupoles are switched off, which can then be used as a diagnostic to find out which of the magnets cause the beam rotation. The first five beam rotation experiments used the same initial beam parameters, given in table 6.12.

In the first experiment, the polarity of all six magnetic quadrupoles was switched in order to check if the beam rotation is in fact due to the focusing magnets. It was found 


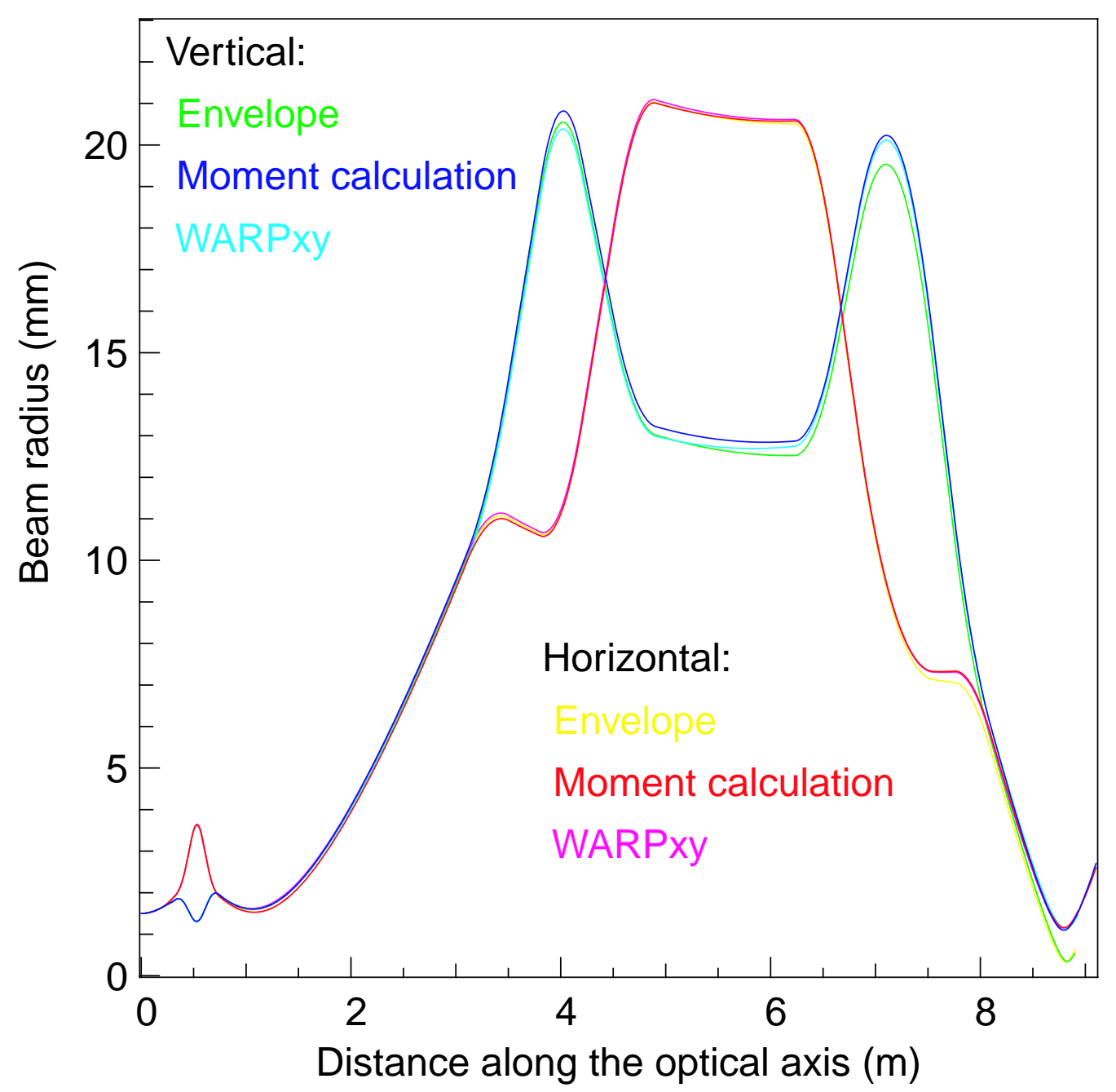

Figure 6.32: The horizontal and vertical beam radius of a $\mathrm{K}-\mathrm{V}$ beam as a function of the longitudinal distance traveled. The envelope solution assumed perfectly aligned magnets, while the moment equations and the 2D transverse slice particle-in-cell simulation (WARPxy) calculated the beam radii in the presence of a $1.24^{\circ}$ rotation of the second magnetic quadrupole.

\begin{tabular}{|l|l|}
\hline Beam energy & $160 \mathrm{keV}$ \\
\hline Initial edge emittance & $1.9 \pi \mathrm{mm} \mathrm{mrad}$ \\
\hline & $92 \mu \mathrm{A}$ second experiment \\
& $95 \mu \mathrm{A}$ third experiment \\
Beam current & $95 \mu \mathrm{A}$ fourth experiment \\
& $93 \mu \mathrm{A}$ fifth experiment \\
\hline Initial beam parameters & $a_{0}=b_{0}=8.0 \mathrm{~mm} ; a_{0}^{\prime}=b_{0}^{\prime}=-3.5 \mathrm{mrad}$ \\
\hline
\end{tabular}

Table 6.12: Beam parameters for the first five beam rotation experiments. 


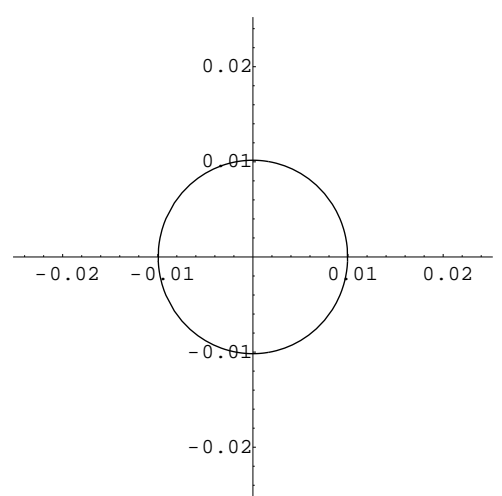

Start of QM1
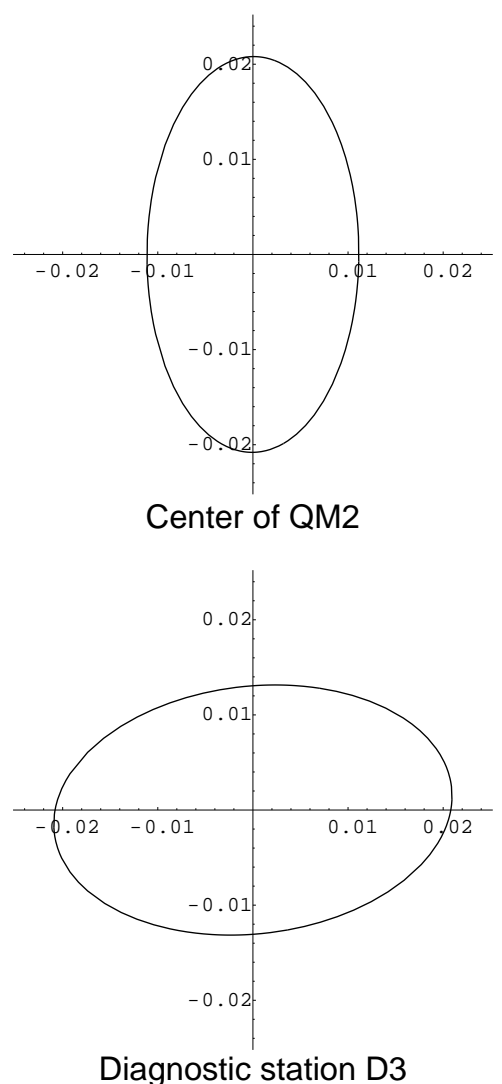

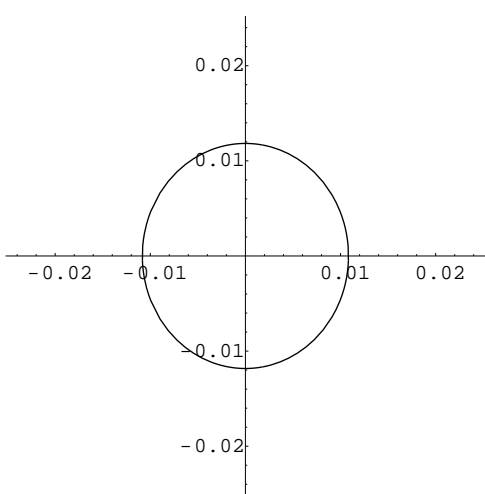

Center of QM1

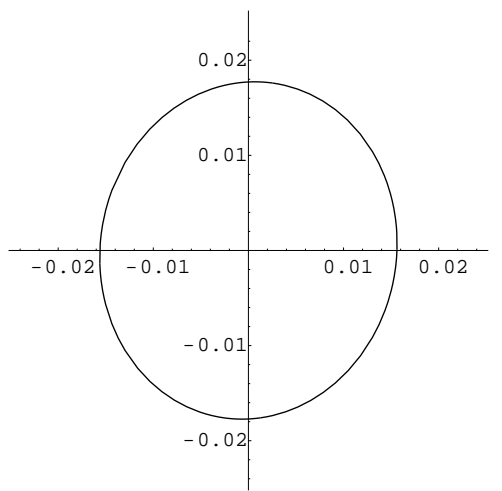

Between QM2 and QM3

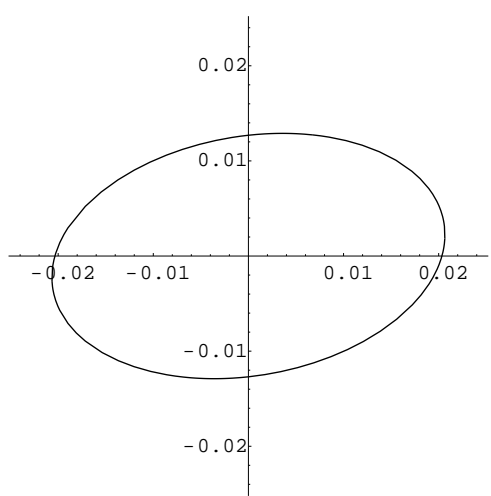

Between QM3 and QM4

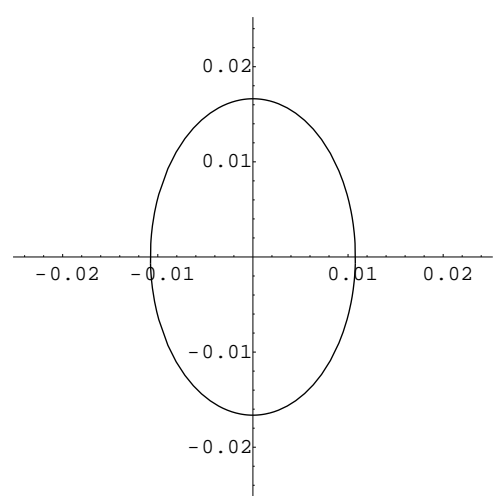

Between QM1 and QM2

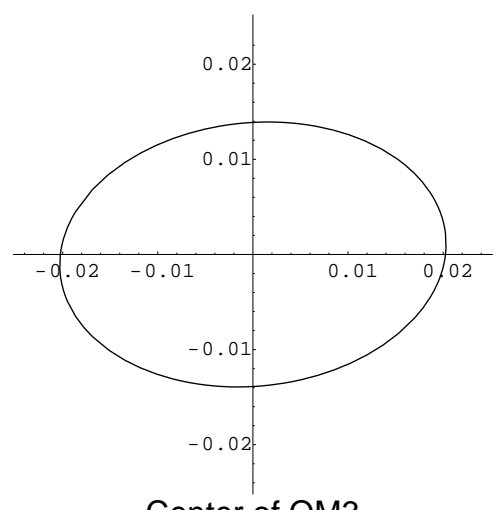

Center of QM3

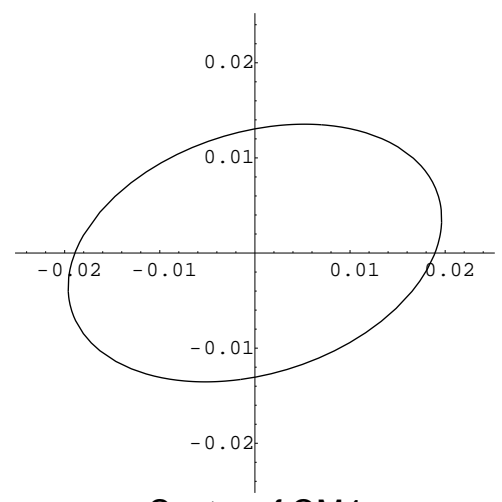

Center of QM4

Figure 6.33: The beam orientation, calculated from the moment equations, at several locations along the lattice, in the presence of a $1.24^{\circ}$ rotation of the second magnetic quadrupole. Continued on next page. 


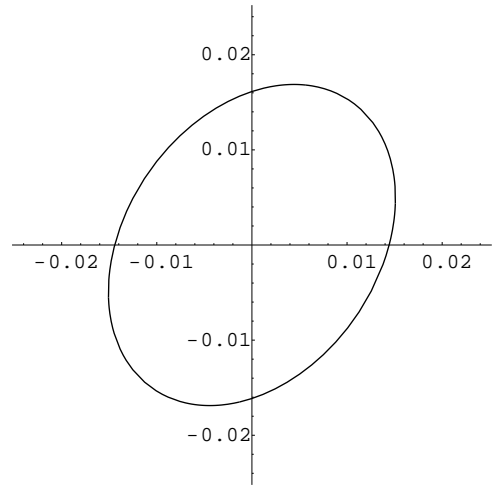

Between QM4 and QM5

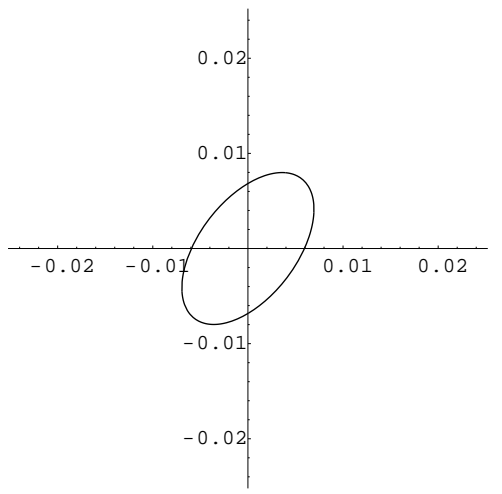

Center of QM6

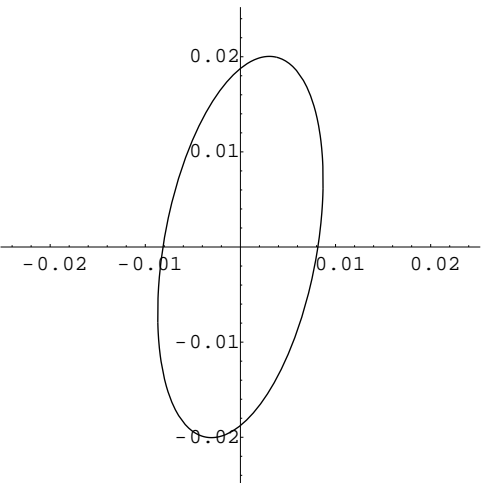

Center of QM5

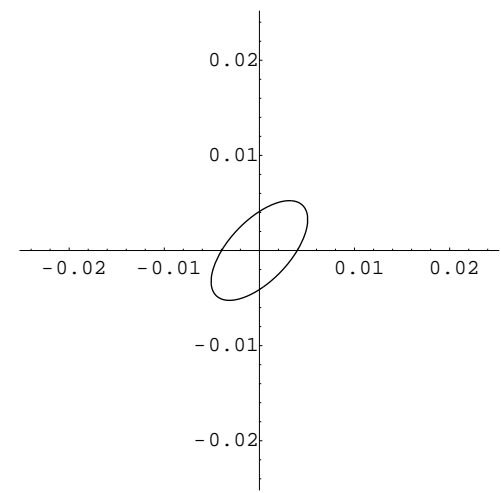

Diagnostic station D4

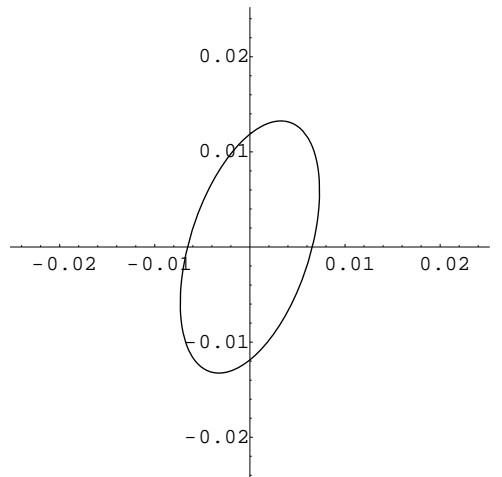

Between QM5 and QM6

Figure 6.34: Continuation of figure 6.33. 


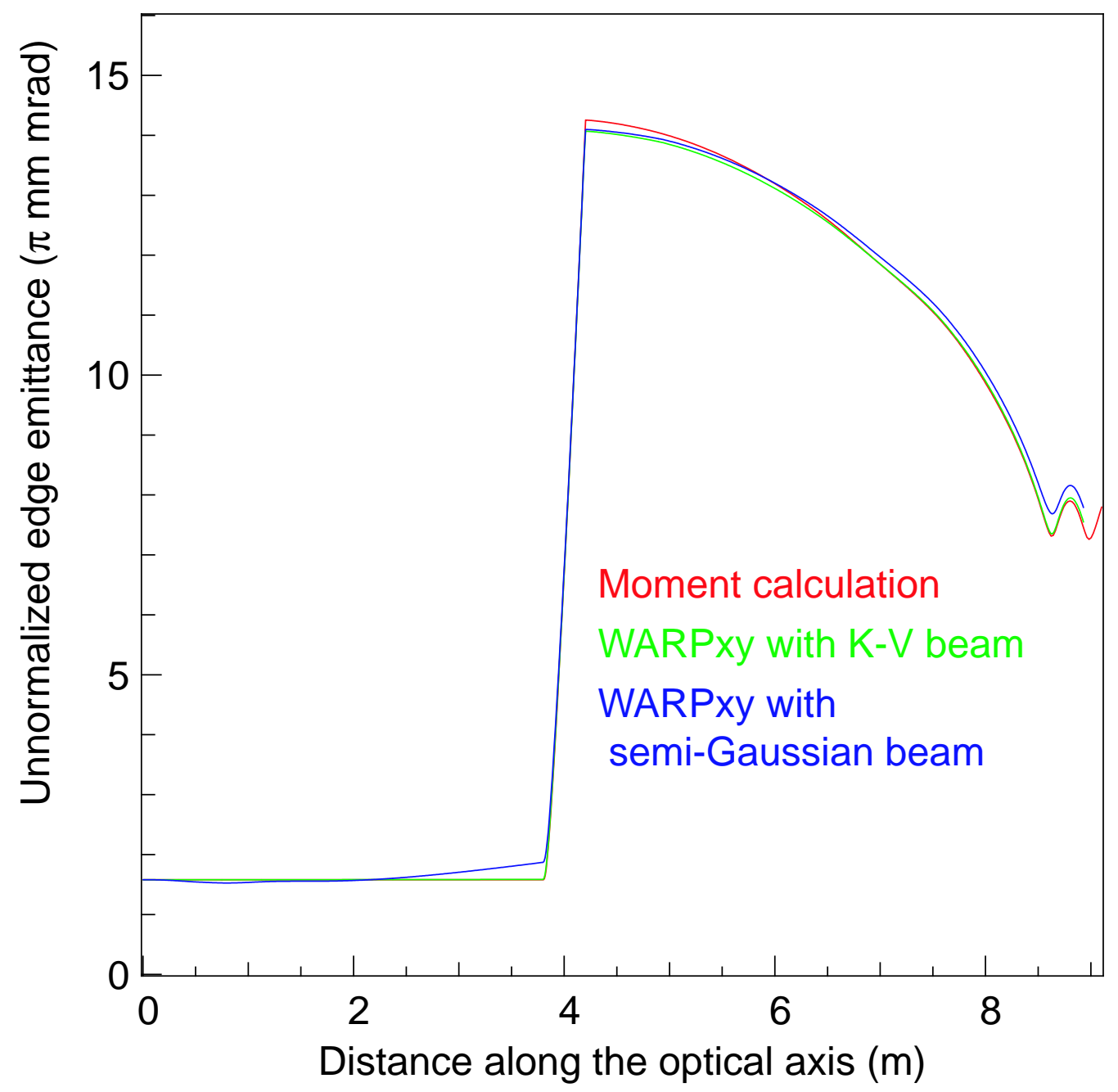

Figure 6.35: The emittance of a K-V and a semi-Gaussian beam as a function of the longitudinal distance traveled, calculated from the moment equations and from a $2 \mathrm{D}$ transverse slice particle-in-cell simulation using WARP. 


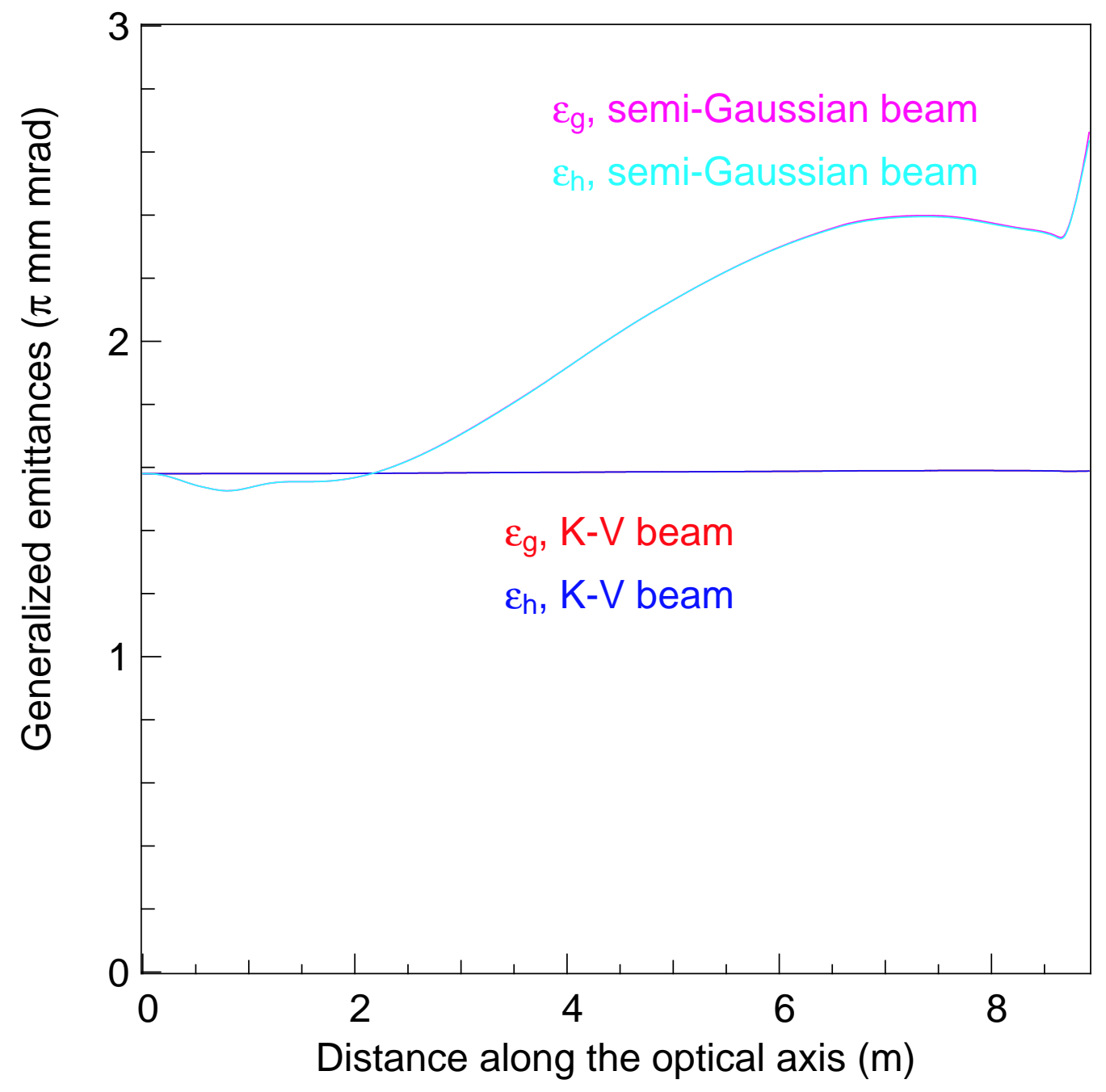

Figure 6.36: The generalized emittance of a $\mathrm{K}-\mathrm{V}$ and a semi-Gaussian beam as a function of the longitudinal distance traveled, calculated from a 2D transverse slice particle-in-cell simulation using WARP. 


\begin{tabular}{|c|c|c|c|}
\hline $\begin{array}{c}\text { Quadrupole center } \\
\text { locations }(\mathrm{m})\end{array}$ & $\begin{array}{c}\text { Quadrupole } \\
\text { lengths }(\mathrm{m})\end{array}$ & $\begin{array}{c}\text { Field } \\
\text { gradient }\end{array}$ & $\begin{array}{c}\text { Aperture } \\
(\mathrm{cm})\end{array}$ \\
\hline \hline 0.127 & 0.1016 & 0. & 2.54 \\
\hline 0.2794 & 0.1016 & 0. & 2.54 \\
\hline 0.4318 & 0.1016 & 0. & 2.54 \\
\hline 0.5842 & 0.1016 & 0. & 2.54 \\
\hline 0.7366 & 0.1016 & 0. & 2.54 \\
\hline 2.5918 & 0.4 & $0 . \mathrm{T} / \mathrm{m}$ & 1.91 \\
\hline 3.2913 & 0.4 & $1.15 \mathrm{~T} / \mathrm{m}$ & 2.54 \\
\hline 3.9948 & 0.4 & $-0.849 \mathrm{~T} / \mathrm{m}$ & 2.54 \\
\hline 5.7178 & 0.4 & $-2.02 \mathrm{~T} / \mathrm{m}$ & 3.18 \\
\hline 6.4798 & 0.717 & $1.95 \mathrm{~T} / \mathrm{m}$ & 3.18 \\
\hline 7.2158 & 0.351 & $-3.15 \mathrm{~T} / \mathrm{m}$ & 1.91 \\
\hline
\end{tabular}

Table 6.13: Lattice properties for the second beam rotation experiment.

that the beam still rotates if the polarity is switched, but in the opposite direction. This sign change indicates that the beam rotation is caused by a physical rotation of the quadrupoles, an asymmetry in their fields, or by the currents in the leads to the quadrupoles.

In the second experiment, the first magnetic quadrupole was switched off. The parameters of the focusing lattice are given in table 6.13. In this experiment, the beam was still rotated at diagnostic station D4. Figure 6.37 shows the current profile of the beam in configuration space as measured in the experiment at diagnostic location D4. The beam was rotated by $-6.1^{\circ}$ with respect to the horizontal axis. It should be noted though that it is difficult to determine the exact rotation angle due to the nonuniformities in the beam profile.

We can conclude though that because the beam was still rotated although the first magnetic quadrupole was switched off, case (a) from table 6.11 can be eliminated. We can assess the cases (b) through (e) by comparing the beam rotation found in the experiment to the beam rotation as calculated from the moments equations. Figures 6.38 


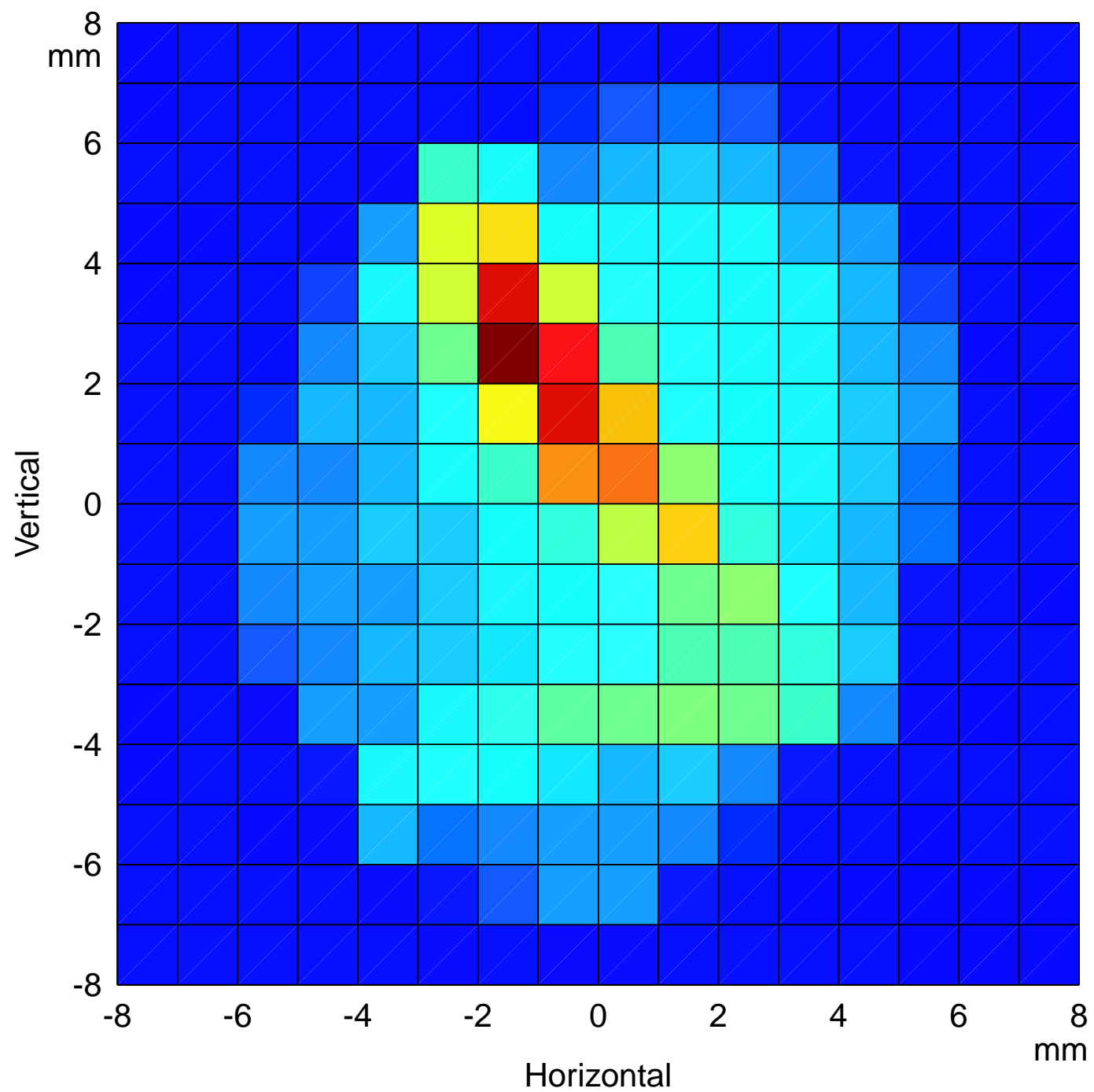

Figure 6.37: Current distribution of the beam as measured in the experiment at diagnostic location $\mathrm{D} 4$, using the focusing lattice given in table 6.13 . The beam was rotated by $-6.1^{\circ}$. 


\begin{tabular}{|c|c|c|c|}
\hline $\begin{array}{c}\text { Quadrupole center } \\
\text { locations }(\mathrm{m})\end{array}$ & $\begin{array}{c}\text { Quadrupole } \\
\text { lengths }(\mathrm{m})\end{array}$ & $\begin{array}{c}\text { Field } \\
\text { gradient }\end{array}$ & $\begin{array}{c}\text { Aperture } \\
(\mathrm{cm})\end{array}$ \\
\hline \hline 0.127 & 0.1016 & 0. & 2.54 \\
\hline 0.2794 & 0.1016 & 0. & 2.54 \\
\hline 0.4318 & 0.1016 & 0. & 2.54 \\
\hline 0.5842 & 0.1016 & 0. & 2.54 \\
\hline 0.7366 & 0.1016 & 0. & 2.54 \\
\hline 2.5918 & 0.4 & $0 . \mathrm{T} / \mathrm{m}$ & 1.91 \\
\hline 3.2913 & 0.4 & $0.99 \mathrm{~T} / \mathrm{m}$ & 2.54 \\
\hline 3.9948 & 0.4 & $0 . \mathrm{T} / \mathrm{m}$ & 2.54 \\
\hline 5.7178 & 0.4 & $-2.18 \mathrm{~T} / \mathrm{m}$ & 3.18 \\
\hline 6.4798 & 0.717 & $1.92 \mathrm{~T} / \mathrm{m}$ & 3.18 \\
\hline 7.2158 & 0.351 & $-2.64 \mathrm{~T} / \mathrm{m}$ & 1.91 \\
\hline
\end{tabular}

Table 6.14: Lattice properties for the third beam rotation experiment.

through 6.41 show the current distribution in configuration space as measured at diagnostic station D4, together with the beam ellipse as calculated from the moment equations for the cases (b) through (e). This allows us to compare the measured beam rotation angle with the calculated rotation angles for the cases listed in table 6.11, as well as to determine the sign of the rotation angle. Whereas the beam rotation angles found from the moment equations agree rather well with the value found experimentally, it should be noted that the measurement of the rotation angle is obscured by the nonuniformities in the current profile.

In the third experiment, the first and third magnetic quadrupole were kept at zero field. The parameters of the focusing lattice are given in table 6.14. Also in this experiment the beam was rotated at diagnostic station D4. Figure 6.42 shows the current distribution of the beam as measured in the experiment at diagnostic location D4. Even though the third magnet was now switched off, the beam was still rotated by $-18.4^{\circ}$. So we can also eliminate case (c) from table 6.11.

Figures 6.43 through 6.45 show the current distribution in configuration space as 


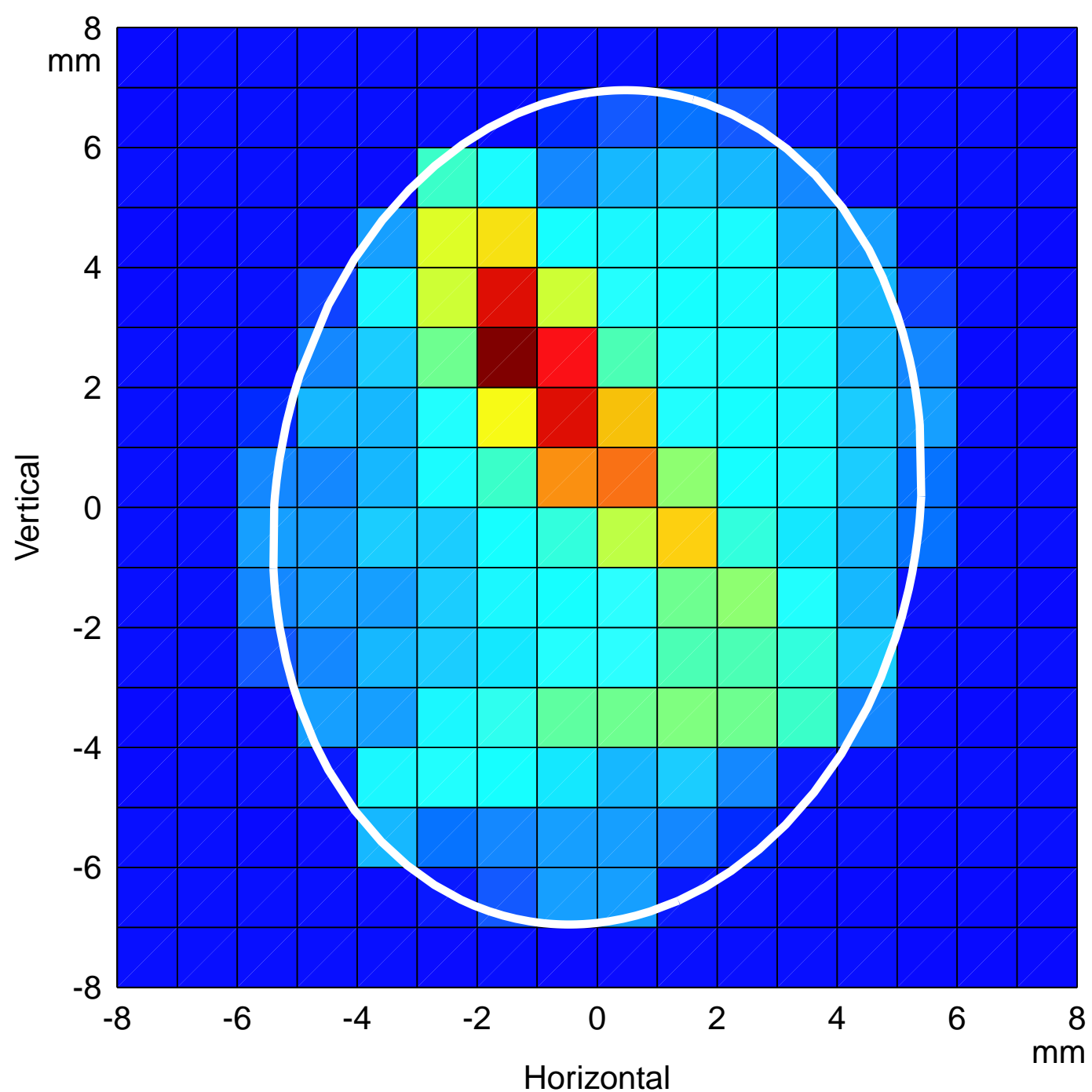

Figure 6.38: Current distribution of the beam as measured in the experiment at diagnostic location $\mathrm{D} 4$, together with the beam ellipse as calculated from the moment equations assuming that the second quadrupole is rotated (case (b) in table 6.11). The moment equations gave a rotation angle of $-9.6^{\circ}$, compared to $-6.1^{\circ}$ in the experiment. 


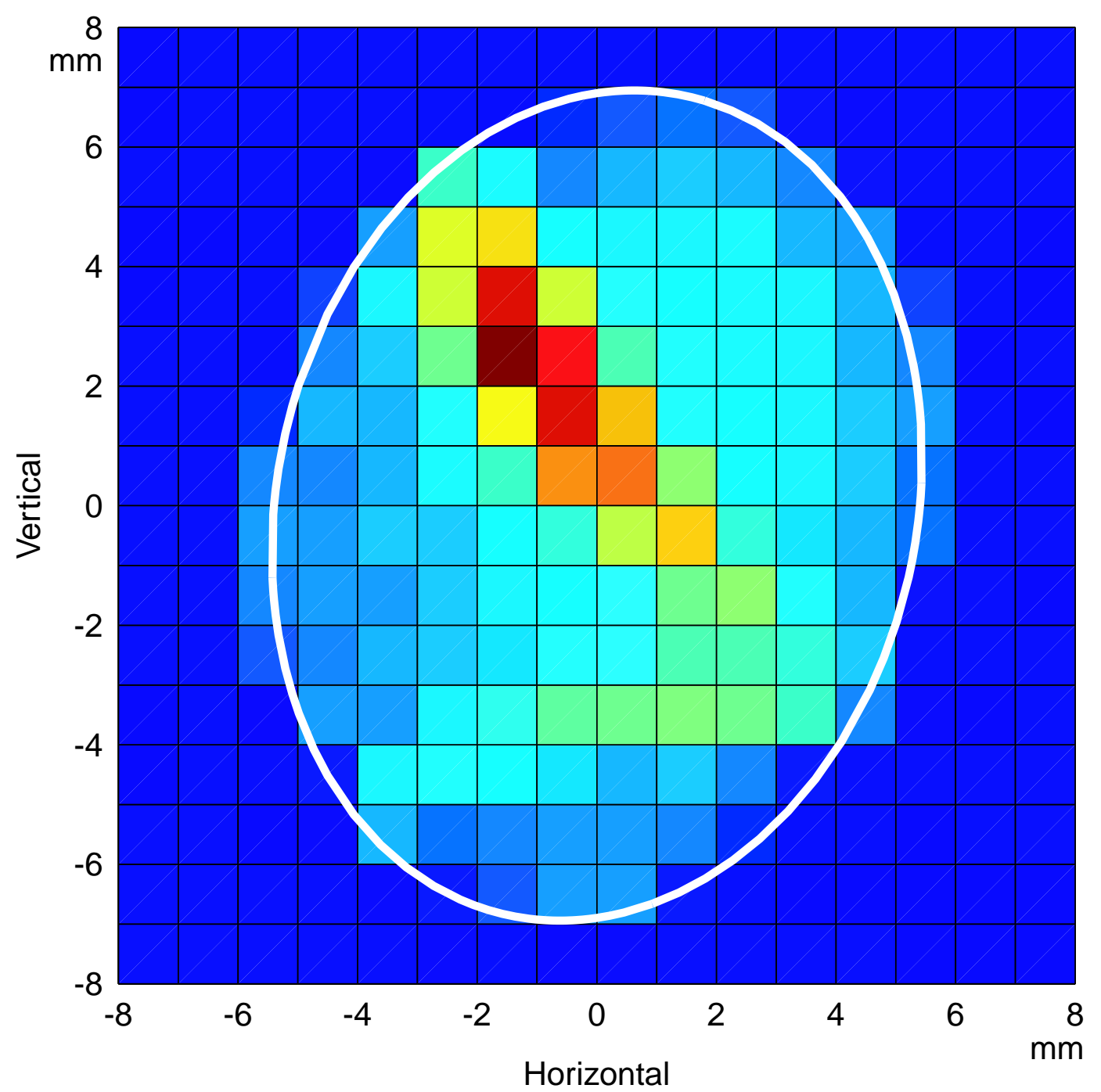

Figure 6.39: Current distribution of the beam as measured in the experiment at diagnostic location $\mathrm{D} 4$, together with the beam ellipse as calculated from the moment equations assuming that the third quadrupole is rotated (case (c) in table 6.11). The moment equations gave a rotation angle of $-12.5^{\circ}$, compared to $-6.1^{\circ}$ in the experiment. 


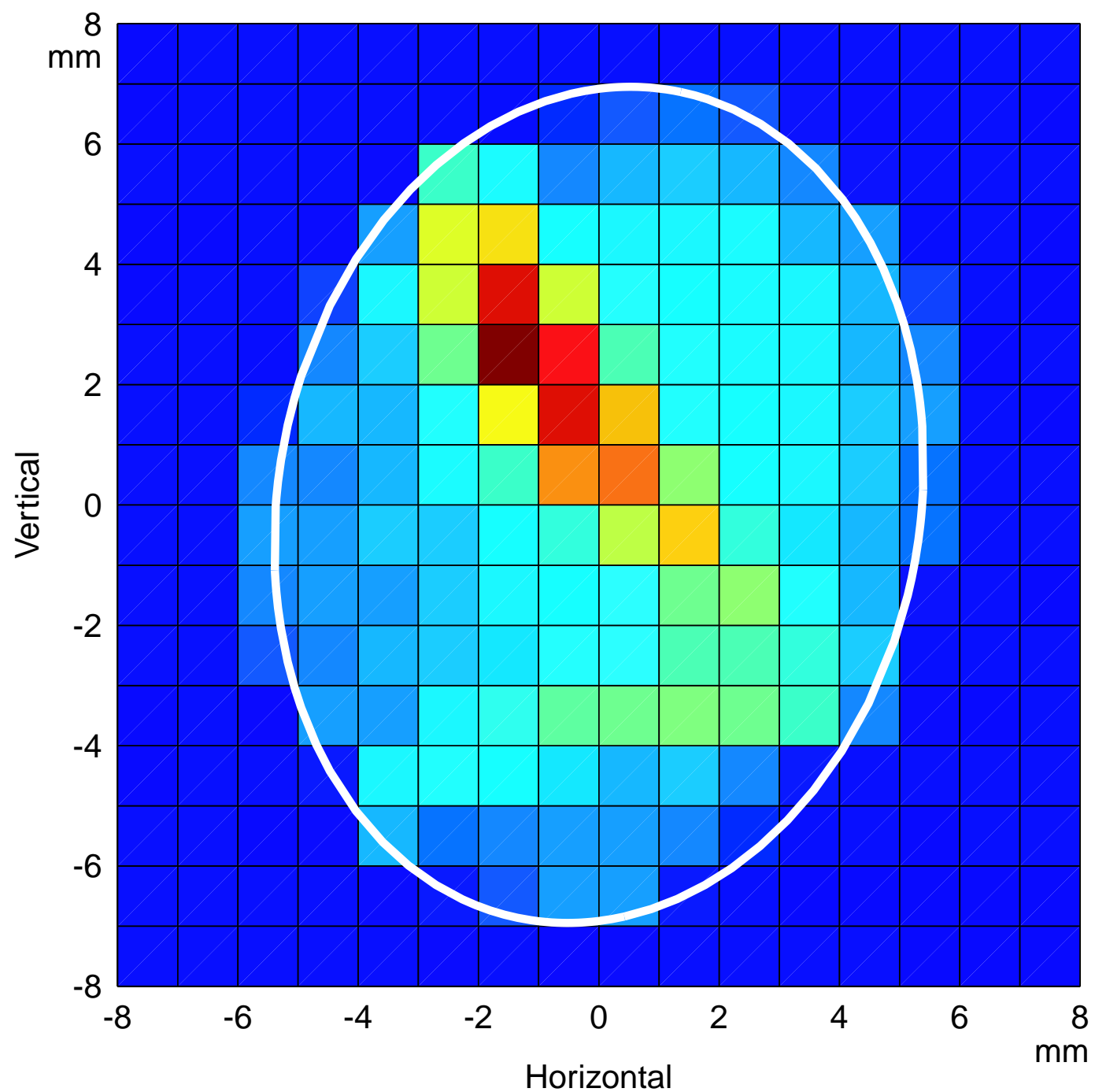

Figure 6.40: Current distribution of the beam as measured in the experiment at diagnostic location D4, together with the beam ellipse as calculated from the moment equations assuming that the first, second, and third quadrupole are rotated (case (d) in table 6.11). The moment equations gave a rotation angle of $-10.6^{\circ}$, compared to $-6.1^{\circ}$ in the experiment. 


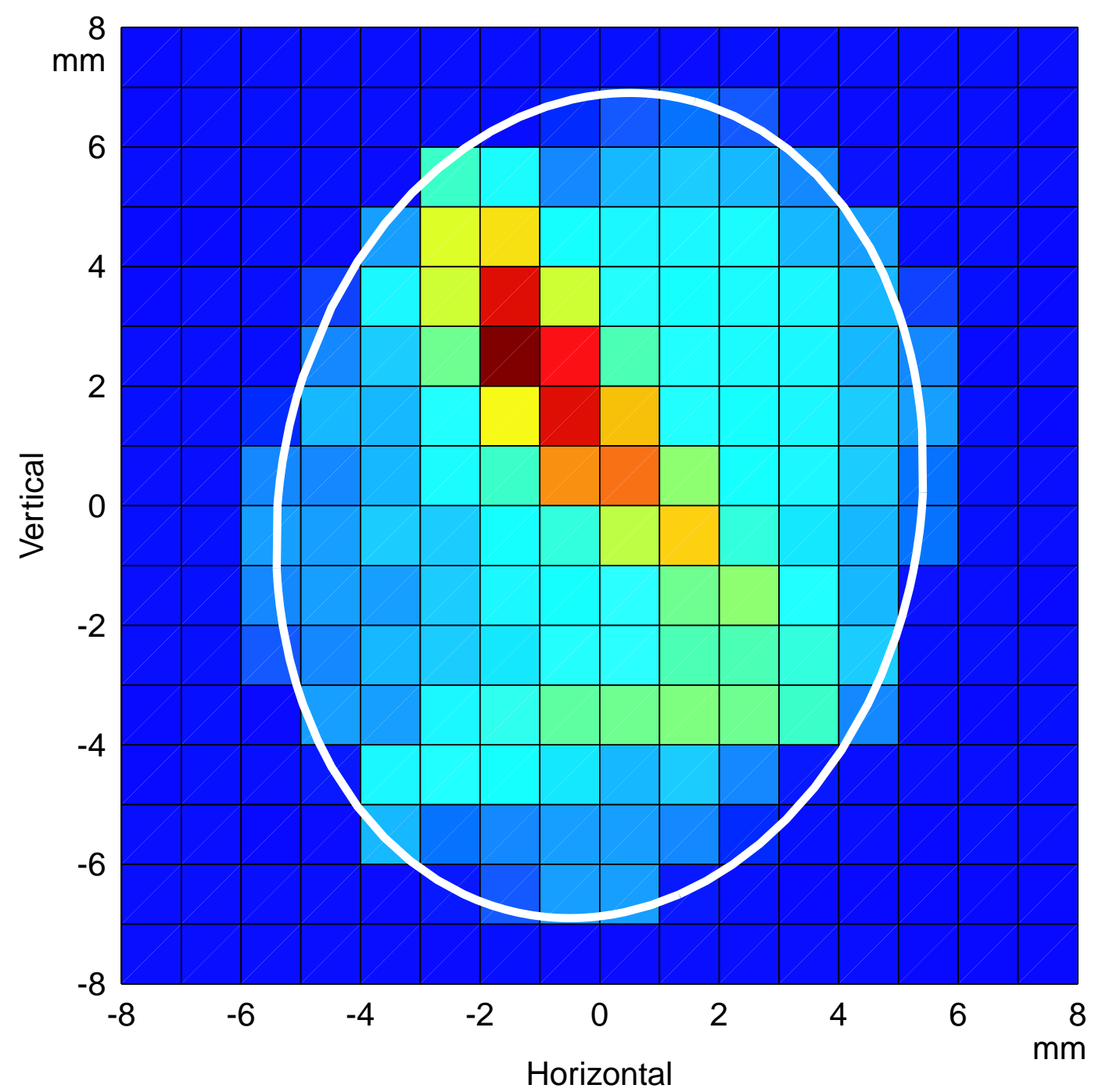

Figure 6.41: Current distribution of the beam as measured in the experiment at diagnostic location $\mathrm{D} 4$, together with the beam ellipse as calculated from the moment equations assuming that all quadrupoles are rotated (case (e) in table 6.11). The moment equations gave a rotation angle of $-10.2^{\circ}$, compared to $-6.1^{\circ}$ in the experiment. 


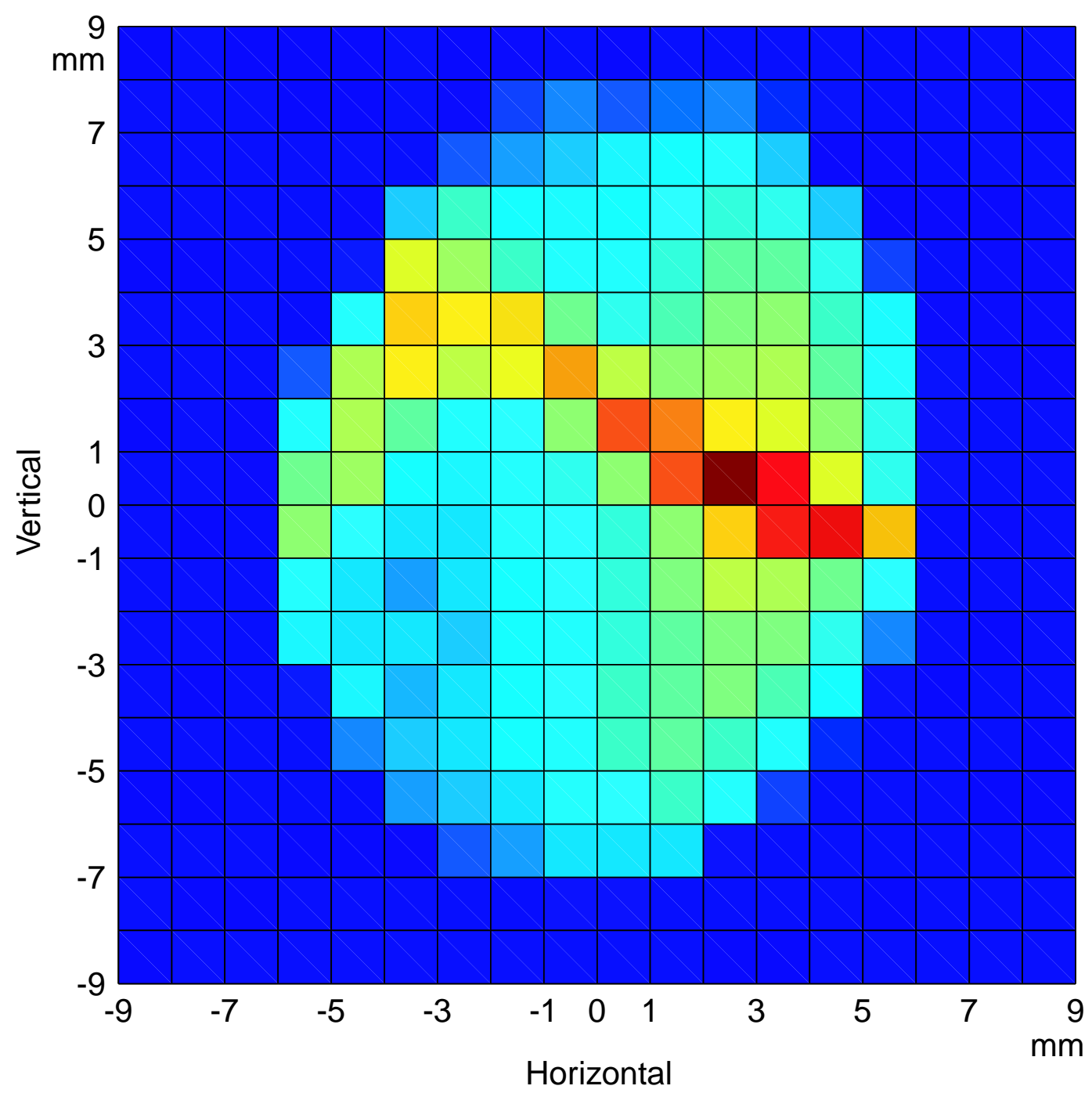

Figure 6.42: Current distribution of the beam as measured in the experiment at diagnostic location $\mathrm{D} 4$, using the focusing lattice given in table 6.14 . The beam was rotated by $-18.4^{\circ}$. 


\begin{tabular}{|c|c|c|c|}
\hline $\begin{array}{c}\text { Quadrupole center } \\
\text { locations }(\mathrm{m})\end{array}$ & $\begin{array}{c}\text { Quadrupole } \\
\text { lengths }(\mathrm{m})\end{array}$ & $\begin{array}{c}\text { Field } \\
\text { gradient }\end{array}$ & $\begin{array}{c}\text { Aperture } \\
(\mathrm{cm})\end{array}$ \\
\hline \hline 0.127 & 0.1016 & 0. & 2.54 \\
\hline 0.2794 & 0.1016 & 0. & 2.54 \\
\hline 0.4318 & 0.1016 & 0. & 2.54 \\
\hline 0.5842 & 0.1016 & 0. & 2.54 \\
\hline 0.7366 & 0.1016 & 0. & 2.54 \\
\hline 2.5918 & 0.4 & $0.82 \mathrm{~T} / \mathrm{m}$ & 1.91 \\
\hline 3.2913 & 0.4 & $0 . \mathrm{T} / \mathrm{m}$ & 2.54 \\
\hline 3.9948 & 0.4 & $-0.51 \mathrm{~T} / \mathrm{m}$ & 2.54 \\
\hline 5.7178 & 0.4 & $-1.97 \mathrm{~T} / \mathrm{m}$ & 3.18 \\
\hline 6.4798 & 0.717 & $1.94 \mathrm{~T} / \mathrm{m}$ & 3.18 \\
\hline 7.2158 & 0.351 & $-2.80 \mathrm{~T} / \mathrm{m}$ & 1.91 \\
\hline
\end{tabular}

Table 6.15: Lattice properties for the fourth beam rotation experiment.

measured at diagnostic station D4, together with the beam ellipse as calculated from the moment equations for the cases (b), (d), and (e). The agreement between the rotation angle determined experimentally and from the moment equations is poor. Although the lattice parameters used in the moment equations are the same as those used in the experiment, even the envelope solution does not agree, as we can see from the difference in the beam size in figures 6.43 through 6.45 .

In the fourth beam rotation experiment, the second magnetic quadrupole was kept at zero field. The parameters of the focusing lattice are given in table 6.15. Again the current profile was measured at diagnostic station D4, but now the beam did not appear to be rotated. It should be noted though that since the beam was almost round at D4, it would be hard to notice a rotation of the beam. Figure 6.46 shows the current distribution of the beam as measured in the experiment at diagnostic location D4.

In order to be able to better distinguish a beam rotation angle if present, in the fifth beam rotation experiment the focusing strength of the fifth magnetic quadrupole in 


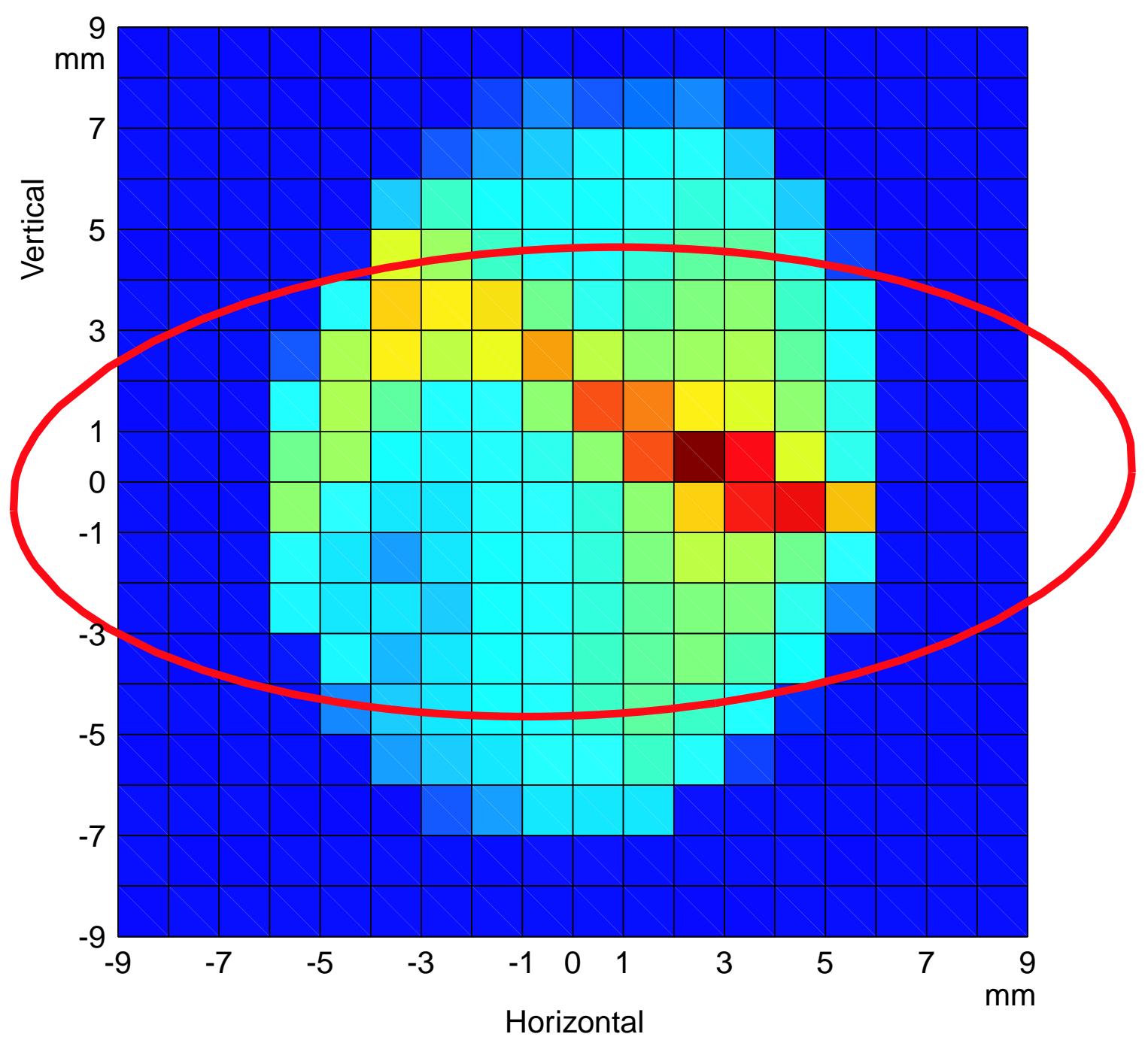

Figure 6.43: Current distribution of the beam as measured in the experiment at diagnostic location D4, together with the beam ellipse as calculated from the moment equations assuming that the second quadrupole is rotated (case (b) in table 6.11). The moment equations gave a rotation angle of $2.4^{\circ}$, compared to $-18.4^{\circ}$ in the experiment. 


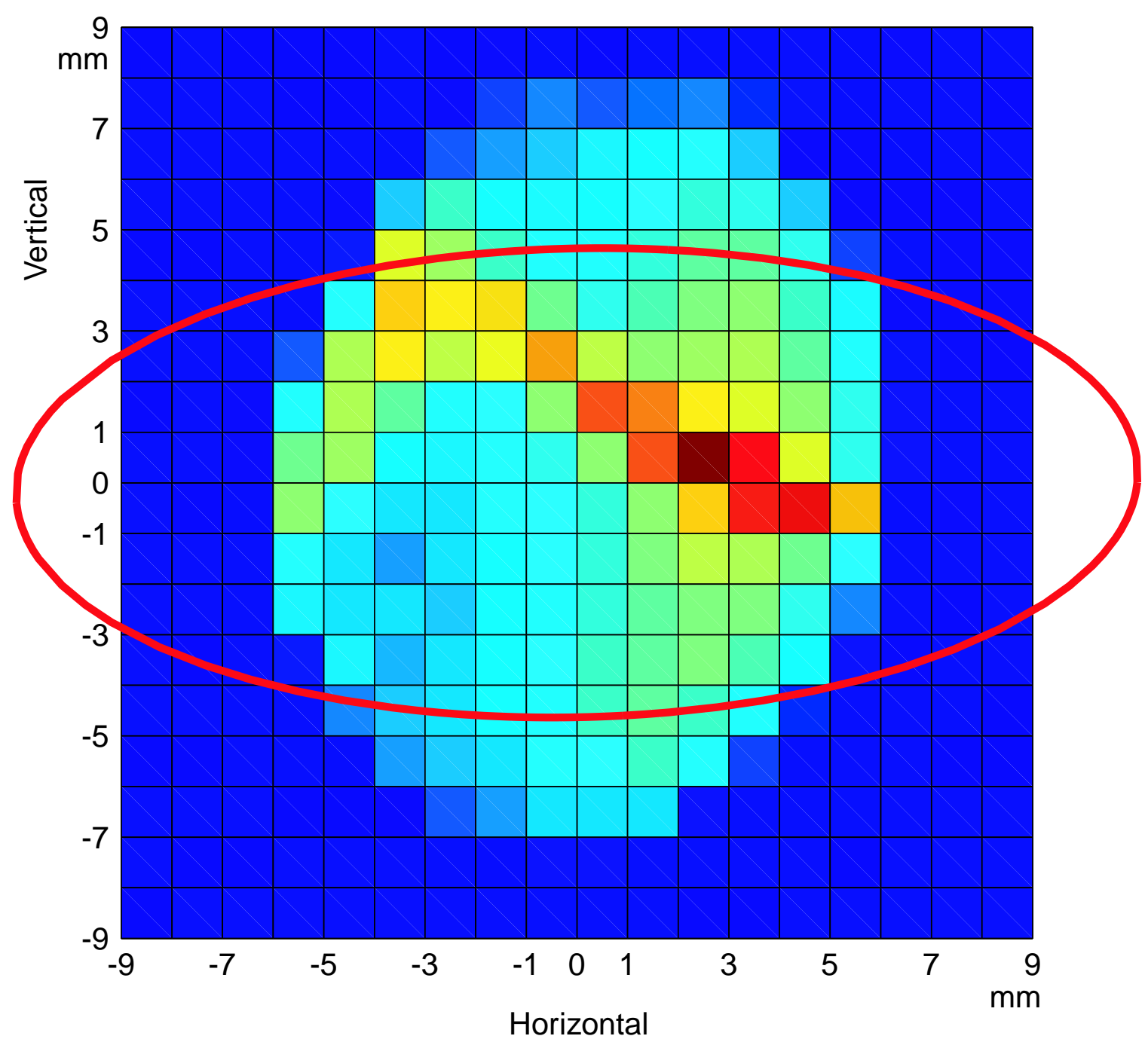

Figure 6.44: Current distribution of the beam as measured in the experiment at diagnostic location D4, together with the beam ellipse as calculated from the moment equations assuming that the first, second, and third quadrupole are rotated (case (d) in table 6.11). The moment equations gave a rotation angle of $1.3^{\circ}$, compared to $-18.4^{\circ}$ in the experiment. 


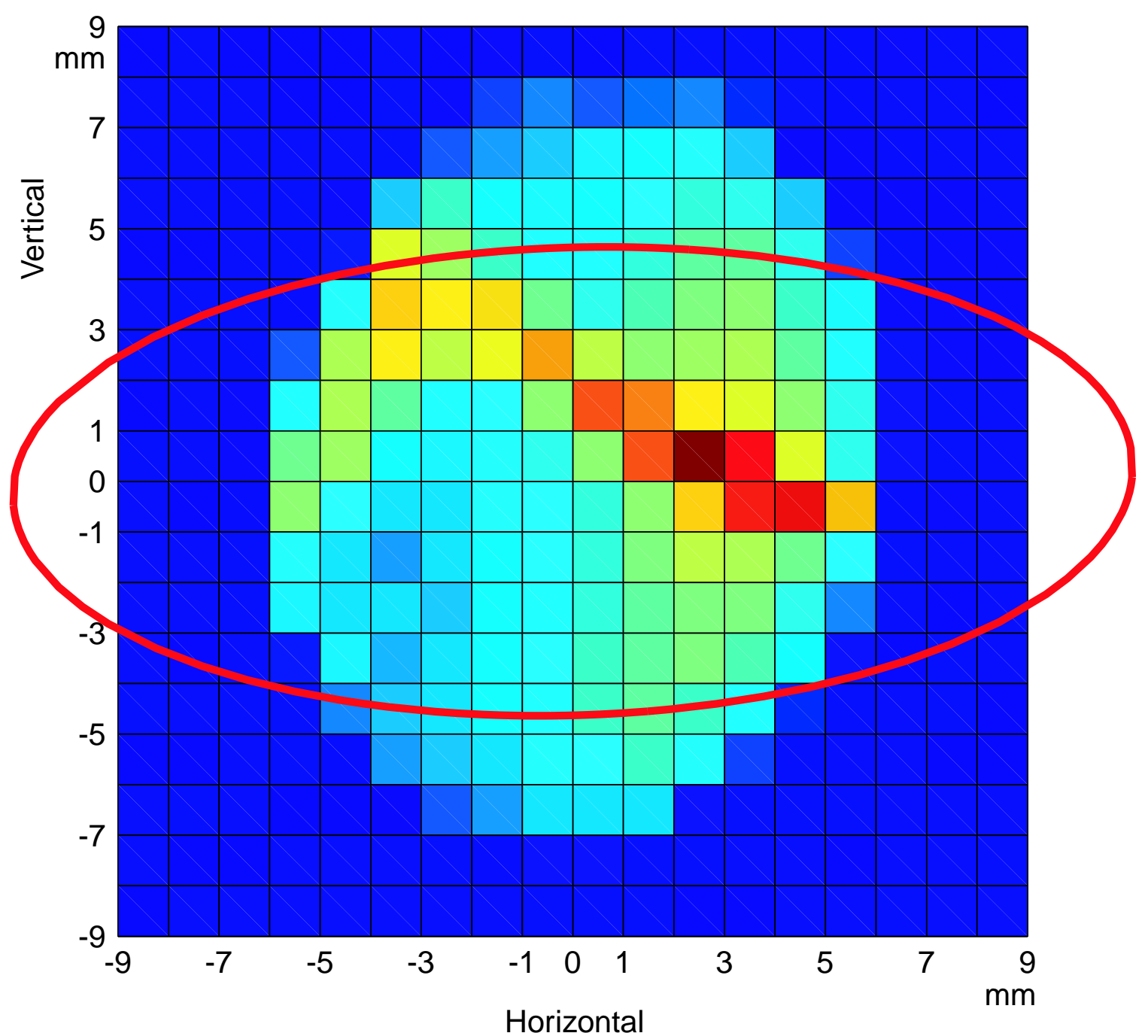

Figure 6.45: Current distribution of the beam as measured in the experiment at diagnostic location D4, together with the beam ellipse as calculated from the moment equations assuming that all six quadrupoles are rotated (case (e) in table 6.11). The moment equations gave a rotation angle of $1.8^{\circ}$, compared to $-18.4^{\circ}$ in the experiment. 


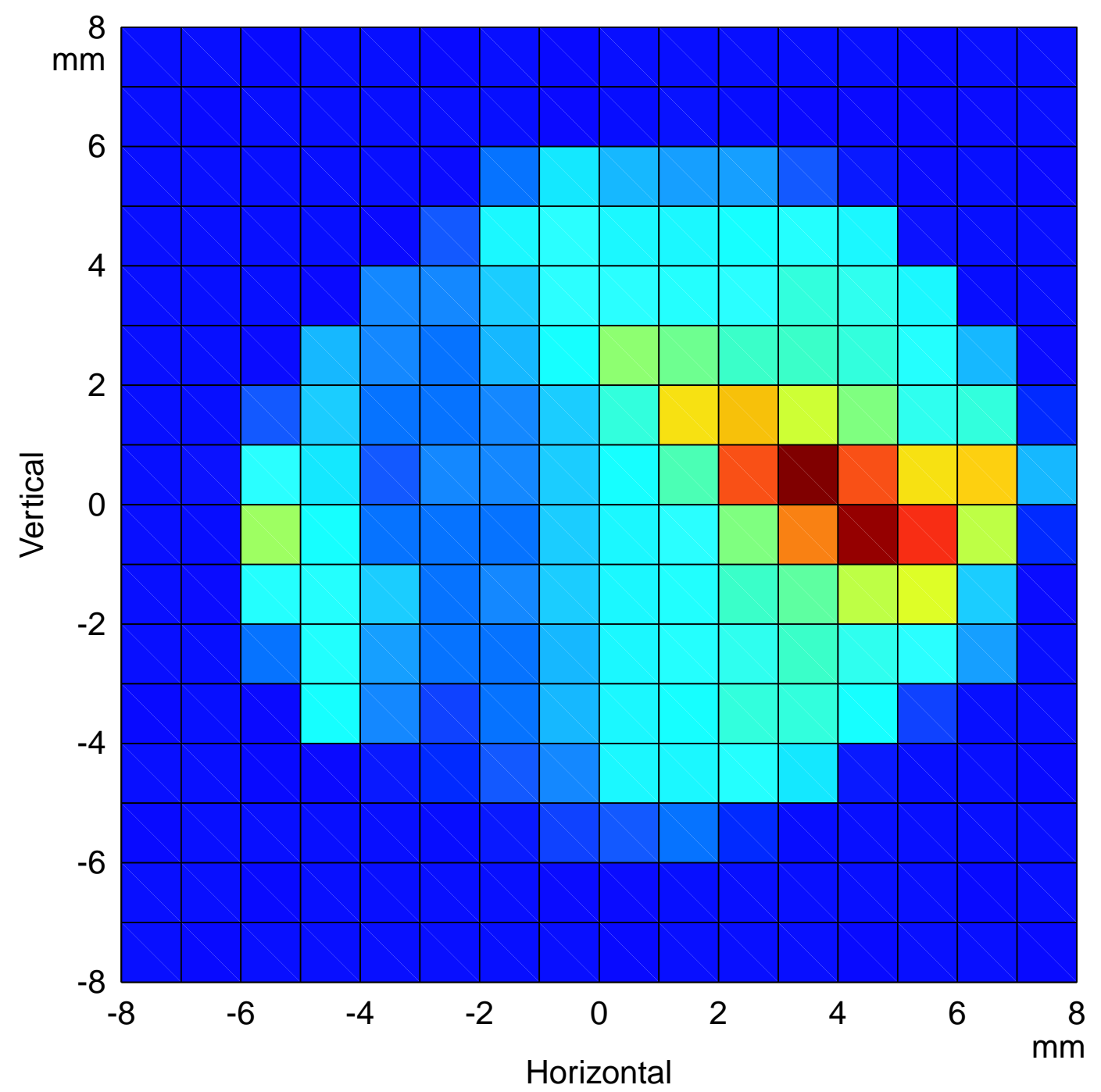

Figure 6.46: Current distribution of the beam as measured in the experiment at diagnostic station D4, using the focusing lattice given in table 6.15. 


\begin{tabular}{|l|l|}
\hline Beam energy & $160 \mathrm{keV}$ \\
\hline Initial edge emittance & $1.9 \pi \mathrm{mm} \mathrm{mrad}$ \\
\hline Beam current & $83.5 \mu \mathrm{A}$ second experiment \\
\hline Initial beam parameters & $a_{0}=b_{0}=4.0 \mathrm{~mm} ; a_{0}^{\prime}=b_{0}^{\prime}=-1.5 \mathrm{mrad}$ \\
\hline
\end{tabular}

Table 6.16: Beam parameters for the beam rotation experiment using a smaller initial beam.

table 6.15 was changed to $1.83 \mathrm{~T} / \mathrm{m}$ such that the beam is elliptical at diagnostic station $\mathrm{D} 4$. The measured current profile is shown in figure 6.47. Again the beam appears to be upright, although its orientation is hard to determine due to the non-uniform charge distribution. Calculating the rotation angle from these data using equation (6.23) yields $-5.0^{\circ}$.

Figures 6.48 and 6.49 show the current profile measured in the experiment, together with the beam ellipse as determined from the moment equations for case (d) and (e) in table 6.11. For case (e), all magnets with non-zero focusing fields are rotated the same degree. Since the beam is round when entering the magnetic section, no torque is exerted on the beam, and the beam rotation angle is equal to the quadrupole rotation angle. For case $(\mathrm{d})$, the first triplet of magnetic quadrupoles is rotated the same degree, so the beam will exit the first triplet with a rotation angle equal to the quadrupole rotation angle. Since the second triplet is upright, the beam will experience a torque there.

In the scaled HIBALL-II case, a beam rotation and subsequent anomalous emittance increase was observed for a beam that was small at the second diagnostic station and expanded in the drift section between the electrostatic and the magnetic section. In order to investigate the effect of the beam expansion on the rotation angle, for the sixth beam rotation experiment a new focusing solution was used with a smaller beam at D2. Table 6.16 gives the initial beam parameters used for this experiment, whereas table 6.17 contains the lattice description. 


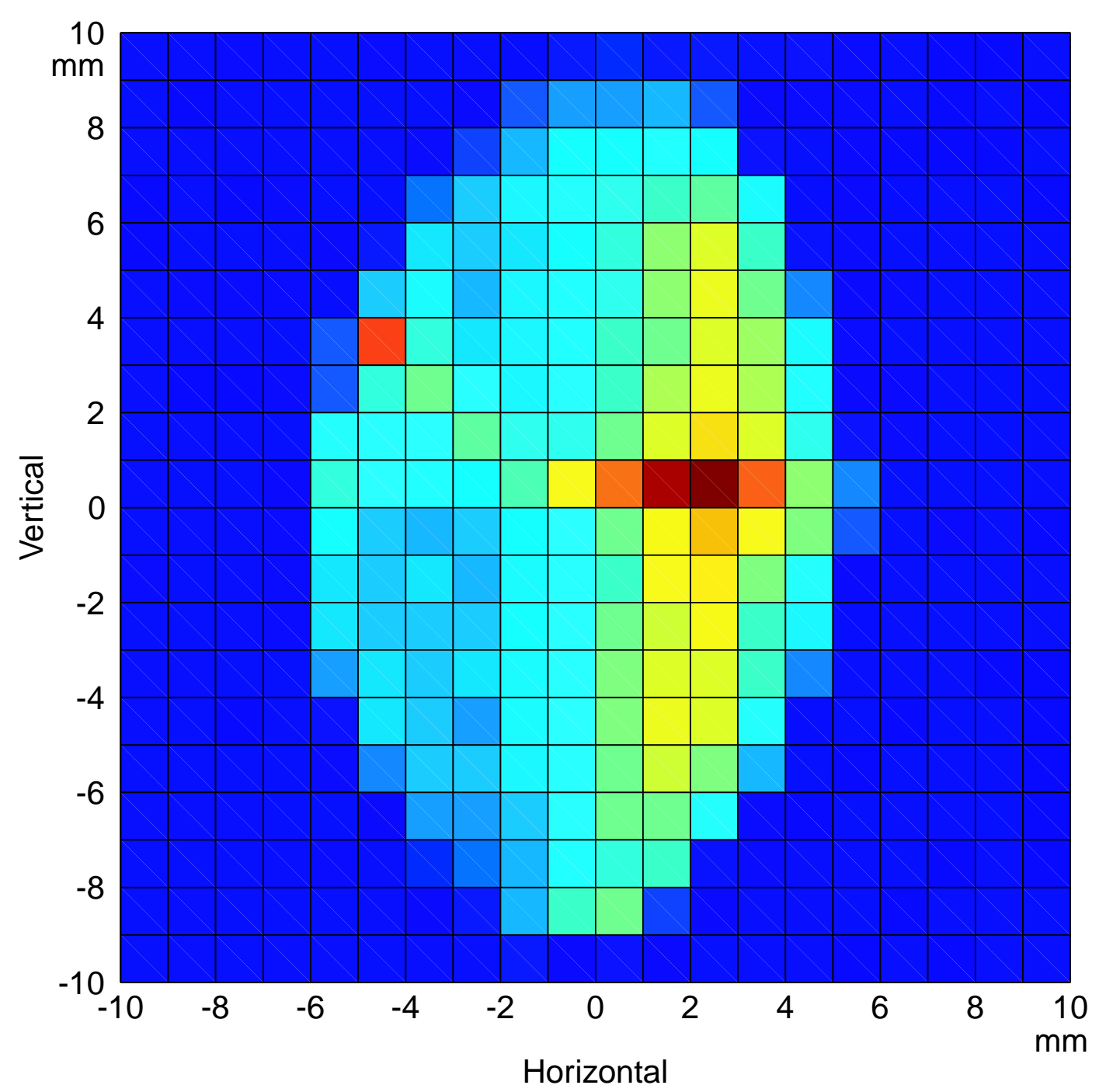

Figure 6.47: Current distribution of the beam as measured in the experiment at diagnostic station D4, using the focusing lattice given in table 6.15 but with a magnetic field gradient of $1.83 \mathrm{~T} / \mathrm{m}$ for the fifth magnetic quadrupole. The beam was rotated by $-5.0^{\circ}$. 


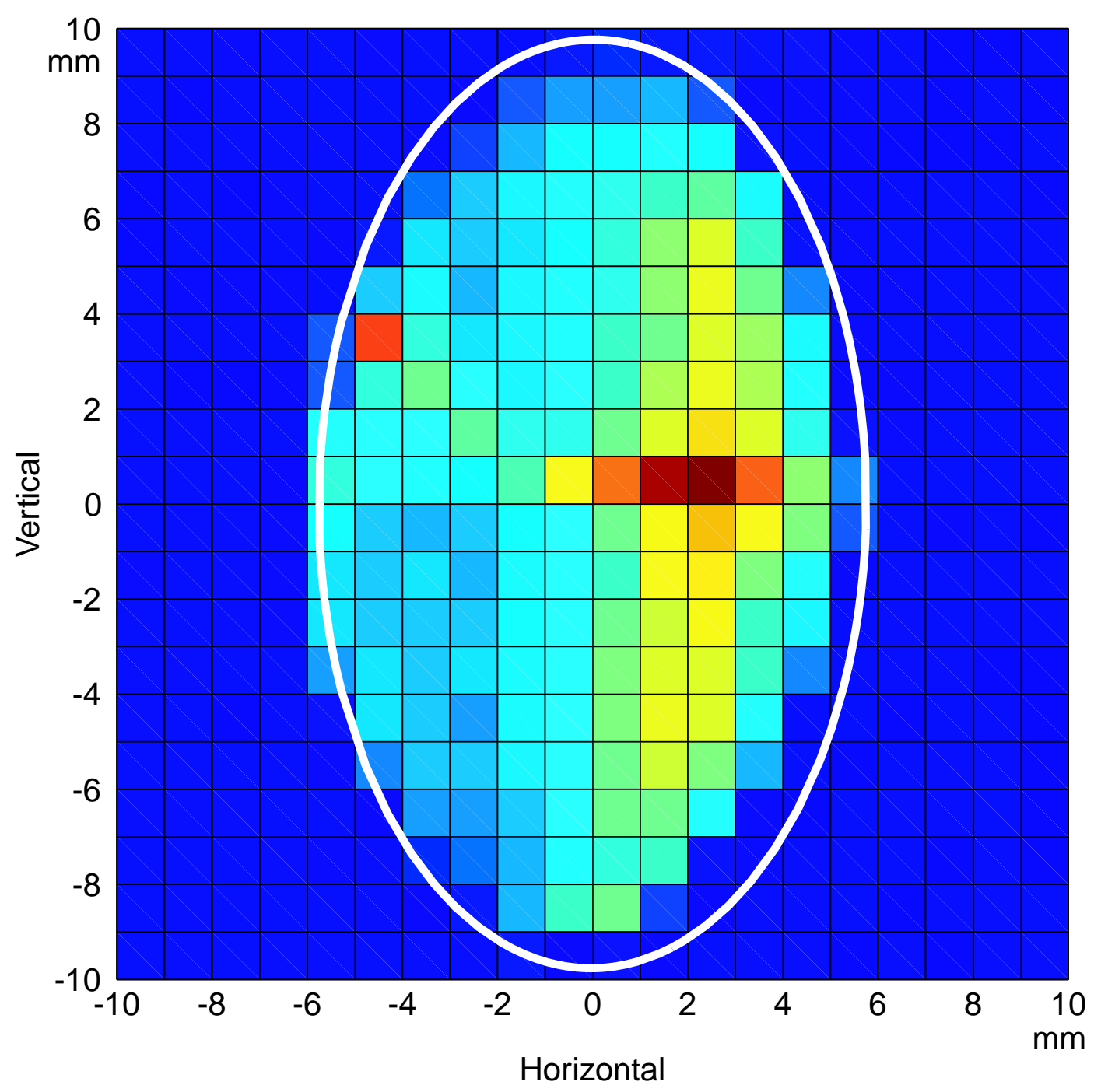

Figure 6.48: Current distribution of the beam as measured in the experiment at diagnostic location $\mathrm{D} 4$, together with the beam ellipse as calculated from the moment equations assuming that the first, second, and third quadrupole are rotated (case (d) in table 6.11). The moment equations gave a rotation angle of $-0.26^{\circ}$, compared to $-5.0^{\circ}$ in the experiment. 


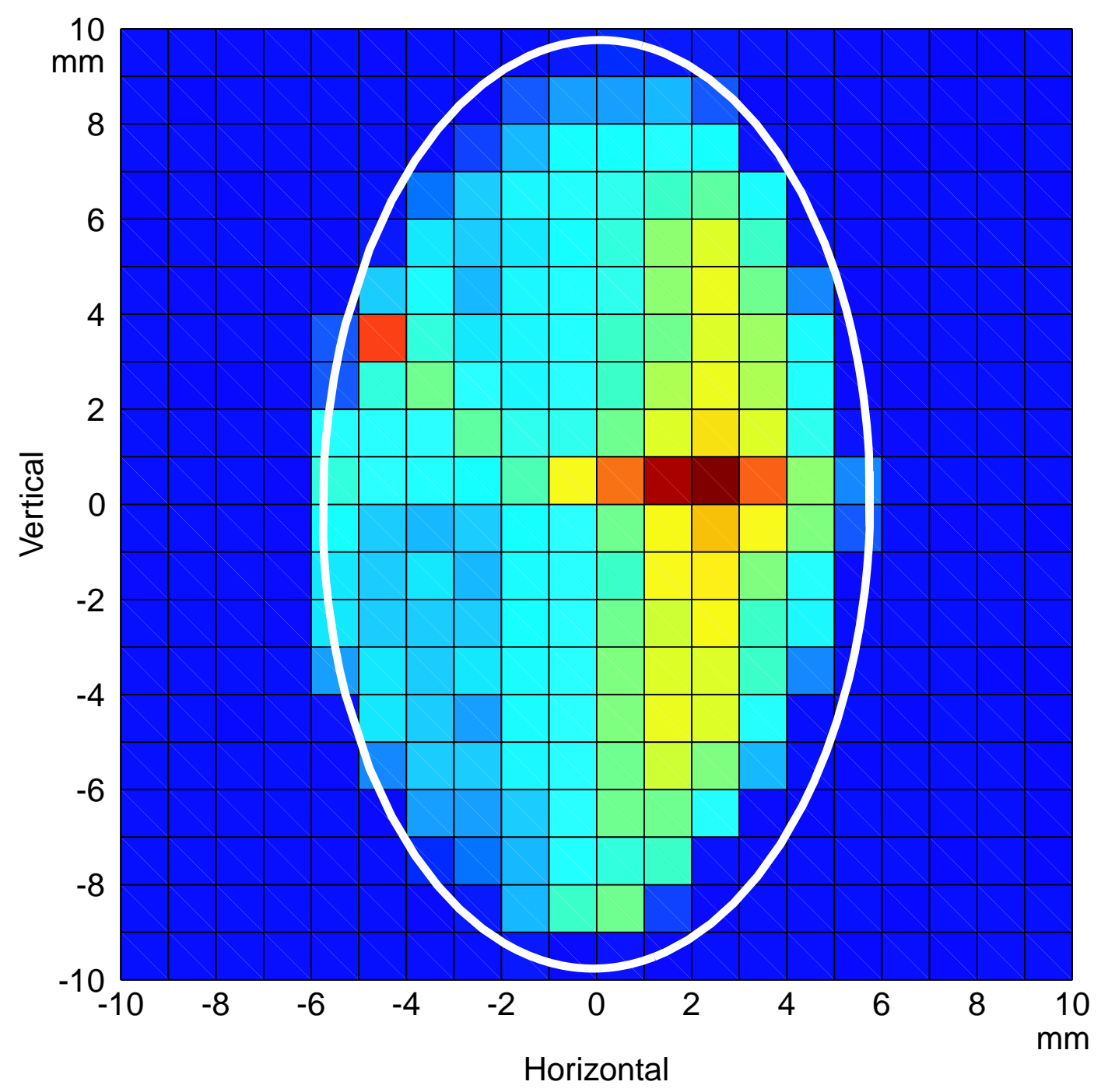

Figure 6.49: Current distribution of the beam as measured in the experiment at diagnostic location D4, together with the beam ellipse as calculated from the moment equations assuming that all six quadrupoles are rotated (case (e) in table 6.11). The moment equations gave a rotation angle of $0.62^{\circ}$ (equal to the quadrupole rotation angle), compared to $-5.0^{\circ}$ in the experiment. 


\begin{tabular}{|c|c|c|c|}
\hline $\begin{array}{c}\text { Quadrupole center } \\
\text { locations }(\mathrm{m})\end{array}$ & $\begin{array}{c}\text { Quadrupole } \\
\text { lengths }(\mathrm{m})\end{array}$ & $\begin{array}{c}\text { Field } \\
\text { gradient }\end{array}$ & $\begin{array}{c}\text { Aperture } \\
(\mathrm{cm})\end{array}$ \\
\hline \hline 0.127 & 0.1016 & 0. & 2.54 \\
\hline 0.2794 & 0.1016 & 0. & 2.54 \\
\hline 0.4318 & 0.1016 & 0. & 2.54 \\
\hline 0.5842 & 0.1016 & 0. & 2.54 \\
\hline 0.7366 & 0.1016 & 0. & 2.54 \\
\hline 2.5918 & 0.4 & $1.22 \mathrm{~T} / \mathrm{m}$ & 1.91 \\
\hline 3.2913 & 0.4 & $0 . \mathrm{T} / \mathrm{m}$ & 2.54 \\
\hline 3.9948 & 0.4 & $-0.90 \mathrm{~T} / \mathrm{m}$ & 2.54 \\
\hline 5.7178 & 0.4 & $-1.47 \mathrm{~T} / \mathrm{m}$ & 3.18 \\
\hline 6.4798 & 0.717 & $1.43 \mathrm{~T} / \mathrm{m}$ & 3.18 \\
\hline 7.2158 & 0.351 & $-1.33 \mathrm{~T} / \mathrm{m}$ & 1.91 \\
\hline
\end{tabular}

Table 6.17: Lattice properties for the beam rotation experiment using the smaller initial beam (table 6.16).

Figure 6.50 shows the current distribution of the beam as measured in the experiment at diagnostic location D4. Since the beam was almost round at diagnostic station D4, it is hard to determine whether the beam was rotated or not.

Figures 6.51 and 6.52 show the current distribution in configuration space as measured at diagnostic station D4, together with the beam ellipse as calculated from the moment equations for the cases (d) and (e). There is good agreement for the beam size and orientation between the experiment and the moments calculation. In the experiment however, the emittance increased from $1.9 \pi \mathrm{mm}$ mrad initially to $9.0 \pi \mathrm{mm}$ mrad horizontally and $5.1 \pi \mathrm{mm}$ mrad vertically, while showing few distortions in phase space. The moment code gave a final emittance of $1.98 \pi \mathrm{mm}$ mrad for case (d) and a negligible emittance increase for case (e). It should be noted though that the magnetic field gradient of the third magnet had to be decreased from from $0.90 \mathrm{~T} / \mathrm{m}$ in the experiment to $0.79 \mathrm{~T} / \mathrm{m}$ in the moment calculation in order to replicate the envelope measured in the experiment.

We can conclude that more than one set of quadrupole rotations can be found 


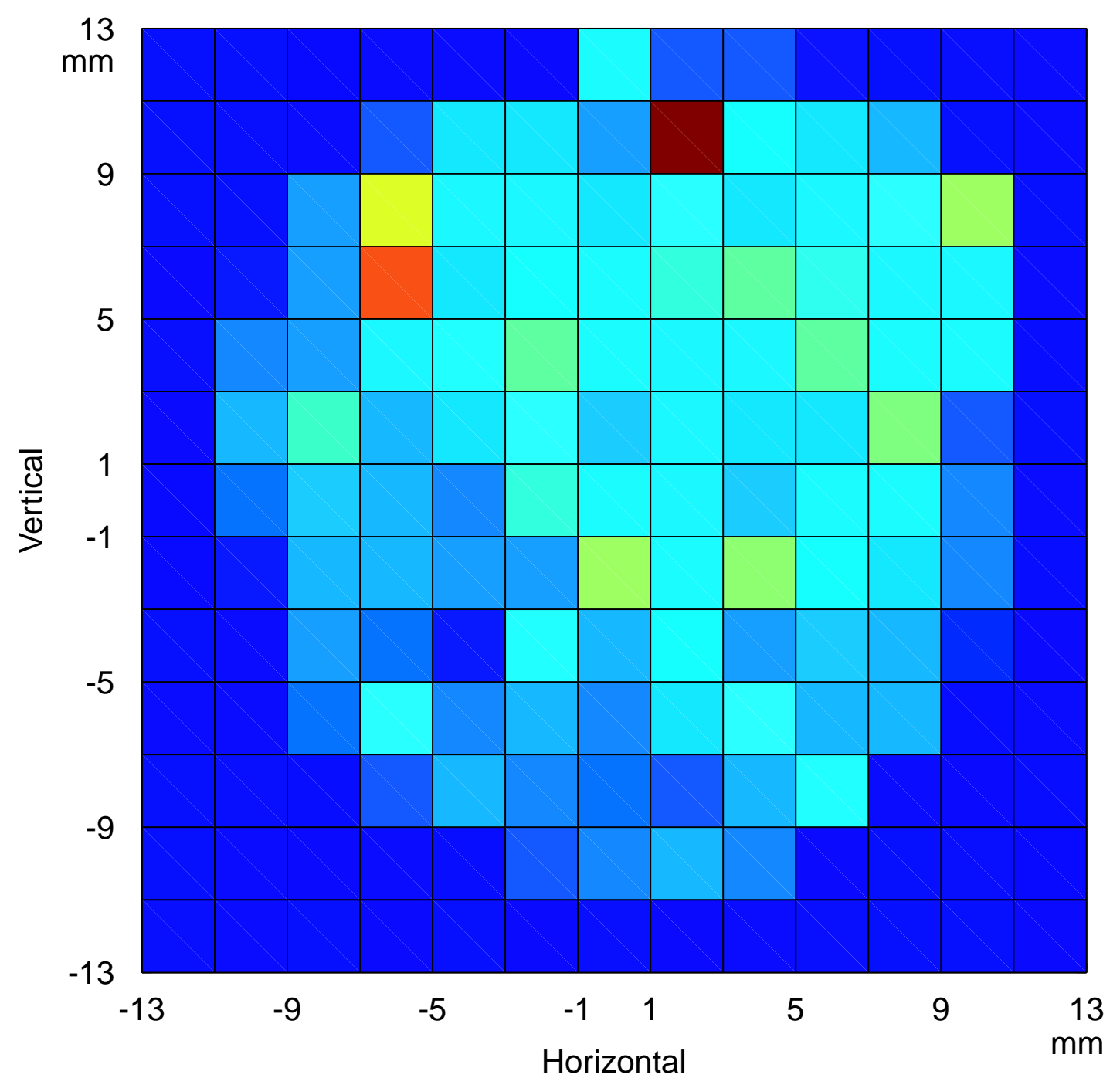

Figure 6.50: Current distribution of the beam as measured in the experiment at diagnostic location D4, using the focusing lattice given in table 6.17. 


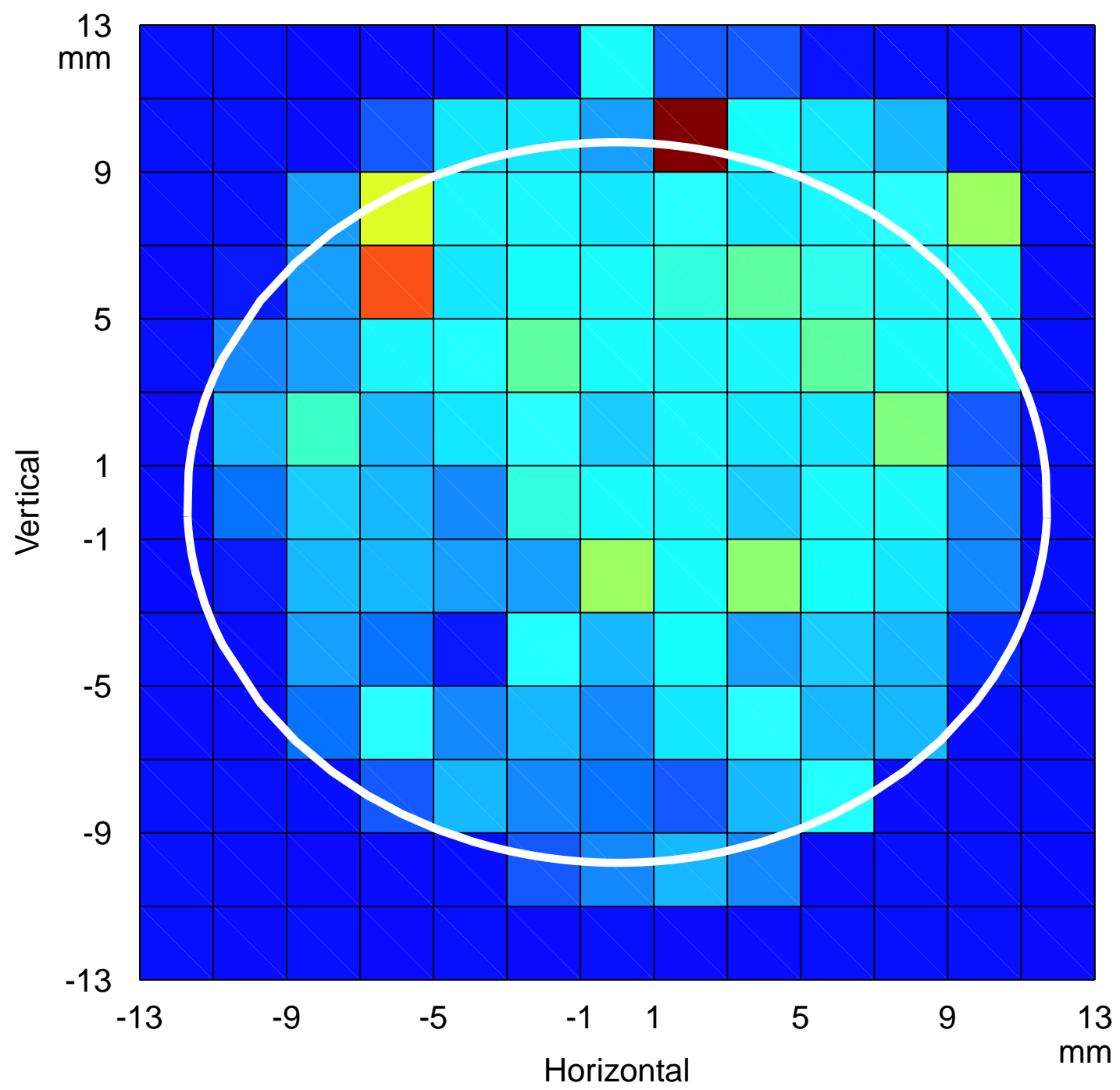

Figure 6.51: Current distribution of the beam as measured in the experiment at diagnostic location D4, together with the beam ellipse as calculated from the moment equations assuming that the second quadrupole is rotated (case (d) in table 6.11). 


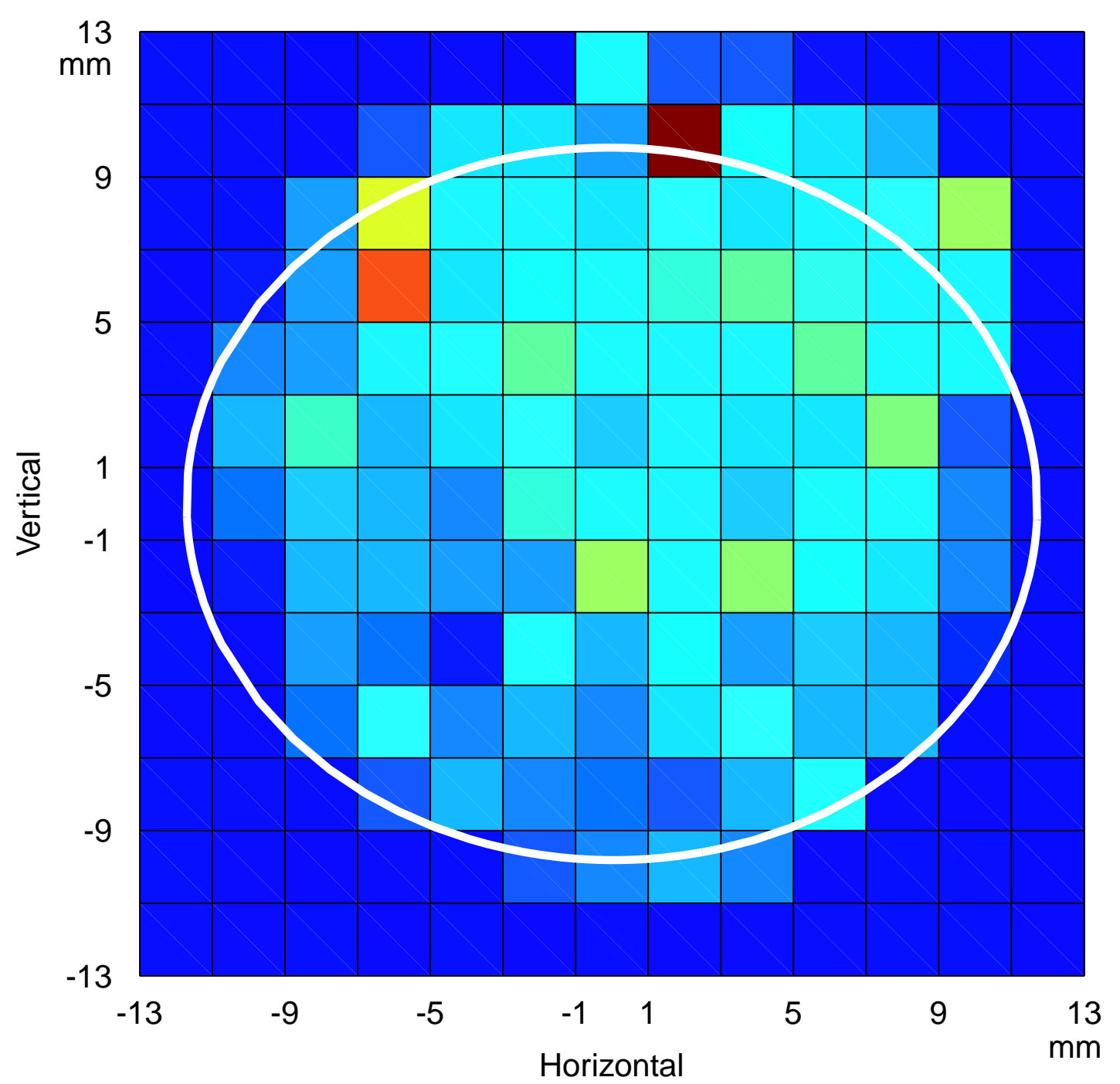

Figure 6.52: Current distribution of the beam as measured in the experiment at diagnostic location D4, together with the beam ellipse as calculated from the moment equations assuming that all six quadrupoles are rotated (case (e) in table 6.11). 
that can explain the beam rotation as well as the anomalous emittance growth found in the experiment. These quadrupole rotations agree with various focusing solutions in the Scaled Final Focus Experiment. However, some of the beam rotation experiments could not be replicated due to discrepancies between an envelope calculation with the focusing strengths used in the experiment and the beam envelope found in the experiment. The calculated beam rotations are consistent though with those beam rotation experiments whose beam envelope could be replicated. Furthermore, the quadrupole rotations needed to generate such a beam rotation are surprisingly small, and might even be generated from small current asymmetries in the quadrupole magnets. Therefore, it cannot be excluded that quadrupole rotations are the cause of the beam rotation and the anomalous emittance growth found in the experiment. This hypothesis can be tested by performing additional experiments with different focusing solutions and check if the beam rotation and emittance growth in the experiment are consistent with the quadrupole rotation angles such as those given in table 6.11. To test the hypothesis of quadrupole rotations directly, the orientation of the quadrupolar field in the experiment can be measured. 


\section{Chapter 7}

\section{Summary}

In the final stages of a heavy ion fusion driver, the beam is compressed longitudinally in the drift compression section to reduce its pulse length and thereby increase the energy deposition rate. Subsequently, the final focus system converges the beam onto a small spot on the target.

To design a drift compression scheme, first a desired final pulse shape and duration at the end of drift compression is chosen, as well as a desired average beam radius as a function of position along the drift compression section. Typically the beam radius needs to be larger toward the final focus system. The beam dynamics are then simulated backwards in time starting at the end of the drift compression section, resulting in a longitudinal expansion of the beam. The simulation is first run over one lattice half period to find the magnitude of the current at the beam center. This is then used, together with the desired beam radius, to calculate the half period length and magnet strength needed to keep the undepressed tune fixed. We iterate over each lattice half period separately until the values 
for its length and quadrupole strength have converged. This process is continued for all preceding half periods until the current has decreased sufficiently to match the beam current at the end of the accelerator.

This procedure will give us the required initial velocity tilt and current profile, as well as a focusing lattice set up for the center of the beam. Usually mismatches develop in other parts of the beam due to a rapidly increasing current there. These mismatches can be avoided by rematching the beam at the beginning of the drift compression section. Although this will cause a mismatch near the end of compression section, its effect is not as severe because it lasts for the last several lattice half periods only.

Once the drift compression lattice is set up, and the required initial beam parameters are known, the effects of rematching at the beginning of drift compression on the beam properties at the end of drift compression can be examined. It was found that longitudinal expansion is only weakly dependent on the transverse beam dynamics, and therefore that the final beam parameters are close to what was originally specified. The sensitivity of the drift compression was analyzed for systematic errors in the initial tilt, and for systematic and random errors in the initial current. In order to do these calculations in the spacecharge dominated regime, a new computer code was written to accurately calculate the longitudinal electric field. This computer code assumes that the beam is round, and uses an existing RZ field solver to find the longitudinal electric field.

The effects of third order aberrations in final focus systems were analyzed. Third order aberrations become more severe as the beam convergence angle becomes larger. A large convergence angle is desirable though in order to achieve smaller spot sizes. It was 
shown that for moderate convergence angles of less than $15 \mathrm{mrad}$, the effects of third order aberrations can be mitigated by rematching the quadrupoles in the final focus section, taking the aberrations into account. The use of correction elements such as octupoles can then be avoided. For larger convergence angles, the third order aberrations cause a significant halo formation, which cannot be corrected for with rematching.

The Scaled Final Focus Experiment at LBNL was simulated with detailed particlein-cell calculations in order to improve our understanding of the experimental results. The effect of nonlinear space-charge forces on radially expanding beams was analyzed. The emittance increase during expansion can be cast in nondimensional form, allowing extrapolation to other parameter regimes. Phase-space comparisons have been performed between the experiment and simulations. Although reasonable agreement was achieved, these comparisons also suggested that some important effects in the experiment may have been neglected in the simulation. One of these effects was the beam rotation that occurs in the experiment. The possibility of a rotation of the focusing quadrupoles being the cause of the beam rotation was investigated. A simplified linear model of the Scaled Final Focus Experiment showed that a minor rotation of one or more of the quadrupoles may have caused such a beam rotation. 


\section{Bibliography}

[1] C. W. Allen. Astrophysical Quantities. The Athlone Press, University of London, second edition, 1963.

[2] Michael Scott Armel. Atomic Processes for Heavy Ion Inertial Fusion. PhD thesis, University of California, Berkeley, 2000.

[3] David Ascher, Paul F. Dubois, Konrad Hinsen, Jim Hugunin, and Travis Oliphant. Numerical Python. Lawrence Livermore National Laboratory, 7000 East Avenue, Livermore, CA 94550-9234, February 15, 2001. UCRL-MA-128569.

[4] B. Badger et al. HIBALL - a conceptual heavy ion beam driven fusion reactor study. Technical Report KfK 3202; UWFDM-450, Kernforschungszentrum Karlsruhe, Institut für Neutronenphysik und Reaktortechnik, Karlsruhe, Germany, 1981.

[5] R. O. Bangerter. The induction approach to heavy ion inertial fusion: Accelerator and target considerations. Il nuovo cimento, 106A(11):1445-1456, November 1993.

[6] Roger O. Bangerter. Ion beam fusion. Philosophical Transactions of the Royal Society of London, Series A, Physical Sciences and Engineering, 357:575-593, 1999. 
[7] J. J. Barnard, L. E. Ahle, R. O. Bangerter, F. M. Bieniosek, C. M. Celata, A. Faltens, A. Friedman, D. P. Grote, I. Haber, E. Henestroza, M. J. L. de Hoon, V. P. Karpenko, R. A. Kishek, J. W. Kwan, E. P. Lee, B. G. Logan, S. M. Lund, W. R. Meier, A. W. Molvik, T. C. Sangster, P. A. Seidl, and W. M. Sharp. Planning for an Integrated Research Experiment. In Nuclear Instruments and Methods in Physics Research, Section A, Amsterdam, Netherlands, 2001. Elsevier. 13th International Symposium on Heavy Ion Inertial Fusion, San Diego, California, held March 13-17, 2000.

[8] John J. Barnard. Emittance growth from rotated quadrupoles in heavy ion accelerators. In Proceedings of the 1995 Particle Accelerator Conference, Dallas, pages 3241-3244, 1995.

[9] S. Bernal, R. A. Kishek, and M. Reiser. Observations and simulations of particle-density oscillations in an apertured, space-charge dominated electron beam. In Proceedings of the 1999 Particle Accelerator Conference, New York, pages 1749-1751, 1999.

[10] Charles K. Birdsall and A. Bruce Langdon. Plasma Physics via Computer Simulation. McGraw-Hill Book Company, 1985.

[11] J. A. Blink, W. J. Hogan, J. Hovingh, W. R. Meier, and J. H. Pitts. The High-Yield Lithium-Injection Fusion-Energy (HYLIFE-I) reactor. Technical Report UCID-53559, Lawrence Livermore National Laboratory, 7000 East Avenue, Livermore, CA 945509234, 1985.

[12] V. O. Brady, A. Faltens, D. Keefe, E. P. Lee, and J. Hovingh. Heavy ion fusion 
system assessment: Final focus and transport model. Technical Report LBL-23040, HIFAN-360, Lawrence Berkeley National Laboratory, July 1987.

[13] K. Brown, E. Colton, S. Fenster, A. Garren, I. Haber, I. Hofmann, J. Lawson, S. Penner, and J. Peterson. Final transport in vacuum - summary. In Proceedings of the Heavy Ion Fusion Workshop, page 377, September 1980. Claremont Hotel, Berkeley, California, held October 29 - November 9, 1979.

[14] K. L. Brown and J. M. Peterson. Chromatic correction for the final transport system. In Proceedings of the Heavy Ion Fusion Workshop, pages 385-387, September 1980. Claremont Hotel, Berkeley, California, held October 29 - November 9, 1979.

[15] Karl L. Brown. A design procedure for correcting second-order geometric and chromatic aberrations in a beam transport system. In Proceedings of the Heavy Ion Fusion Workshop, pages 107-108, October 1977. Brookhaven National Laboratory, Upton, New York, held October 17-21, 1977.

[16] Karl L. Brown and Roger V. Servranckx. First- and second-order charged particle optics. Technical Report SLAC-PUB-3381, SLAC, July 1984. Presented at the 3rd Summer School on High Energy Particle Accelerators, Brookhaven National Laboratory, Upton, New York, July 6-16, 1983.

[17] H. Bruck. Accélérateurs Circulaires de Particules, chapter IX. Institut National des Sciences et Techniques Nucléaires, Presses Universitaires de France, 1966.

[18] H. Bruck. Outline of an aberration corrected optical channel without space charge. 
In Proceedings of the Heavy Ion Fusion Workshop, pages 109-111, October 1977. Brookhaven National Laboratory, Upton, New York, held October 17-21, 1977.

[19] D. A. Callahan-Miller. Simulations of Longitudinal Beam Dynamics of Space-Charge Dominated Beams for Heavy Ion Fusion. PhD thesis, University of California, Davis, December 1994.

[20] D. A. Callahan-Miller and M. Tabak. A distributed radiator, heavy ion target driven by Gaussian beams in a multibeam illumination geometry. Nuclear Fusion, 39(7):883-891, July 1999.

[21] C. M. Celata, M. J. L. de Hoon, and J. J. Barnard. Effects of misalignments on space-charge-dominated heavy ion beams in an IRE. Proceedings of the 1999 Particle Accelerator Conference, New York, pages 1803-1805, 1999.

[22] Eugene Colton. Correction of chromatic and geometric aberrations using sextupoles and octupoles. In Proceedings of the Heavy Ion Fusion Workshop, pages 365-378, September 1978. Argonne National Laboratory, Argonne, Illinois, held September 19 26, 1978.

[23] Eugene Colton. Calculations of major 3rd order geometric aberrations for final transport line. In Proceedings of the Heavy Ion Fusion Workshop, pages 378-384, September 1980. Claremont Hotel, Berkeley, California, held October 29 - November 9, 1979.

[24] K. R. Crandall and D. D.-M. Ho. Scaling experiment for heavy-ion fusion final focusing. Particle Accelerators, 37-38:105-109, 1992. 
[25] M. J. L. de Hoon, S. A. MacLaren, and E. P. Lee. Simulation of the LBNL scaled final focus experiment. In Nuclear Instruments and Methods in Physics Research, Section A, Amsterdam, Netherlands, 2001. Elsevier. 13th International Symposium on Heavy Ion Inertial Fusion, San Diego, California, held March 13-17, 2000.

[26] Thomas James Dolan. Fusion Research, Volume I - Principles. Pergaman Press, New York, 1982.

[27] D. A. Edwards and M. J. Syphers. An Introduction to the Physics of High Energy Accelerators. Wiley Series in Beam Physics and Accelerator Technology. John Wiley \& Sons, Inc., 1993.

[28] Andy Faltens and Ed Lee. Beam clearances - Revision. HIFAR Note 430, Lawrence Berkeley National Laboratory, Accelerator and Fusion Research Division, February 1995.

[29] Rainer Feldbacher. The AEP barnbook DATLIB. Nuclear reaction cross sections and reactivity parameter library and files. Technical Report INDC(AUS)-12/G Vers. 1, International Atomic Energy Agency, International Nuclear Data Committee, IAEA Nuclear Data Section, Wagramerstrasse 5, A-1400 Vienna, Austria, October 1987.

[30] T. Kenneth Fowler. The Fusion Quest. The Johns Hopkins University Press, Baltimore, Maryland, 1997.

[31] A. Friedman, J J. Barnard, M. D. Cable, D. A. Callahan, F. J. Deadrick, D. P. Grote, V. P. Karpenko, H. C. Kirbie, B. G. Logan, S. M. Lund, L. A. Nattrass, M. B. Nelson, M. A. newton, T. C. Sangster, W. M. Sharp, T. J. Fessenden, D. L. Judd, S. Eylon, 
H. A. Hopkins, and D. B. Longinotti. Progress toward a prototype recirculating ion induction accelerator. Technical Report UCRL-LR-105821-95-3, Lawrence Livermore National Laboratory, 7000 East Avenue, Livermore, CA 94550-9234, 1995.

[32] A. Friedman, J. J. Barnard, C. M. Celata, G. D. Craig, M. J. L. de Hoon, A. Faltens, D. P. Grote, E. P. Lee, W. M. Sharp, E. Sonnendrucker, I. Haber, and R. A. Kishek. Beam dynamics studies for heavy ion fusion drivers. Proceedings of the 1999 Particle Accelerator Conference, New York, pages 1830-1832, 1999.

[33] Alex Friedman, David P. Grote, Debra A. Callahan, and A. Bruce Langdon. 3D particle simulation of beams using the WARP code: Transport around bends. Particle Accelerators, 37-38:131-139, 1992.

[34] Alex Friedman, David P. Grote, and Irving Haber. Three-dimensional particle simulation of heavy-ion fusion beams. Physics of Fluids B, 4(7):2203-2210, July 1992.

[35] Fusion Power Associates. HIBALL-II — An improved conceptual heavy ion beam driven fusion reactor study. Technical Report KfK 3840; FPA-84-4; UWFDM-625, Kernforschungszentrum Karlsruhe, Institut für Neutronenphysik und Reaktortechnik, Karlsruhe, Germany, 1985.

[36] A. Garren. Studies of a beam line for transport to a target. In Proceedings of the Heavy Ion Workshop, pages 397-402, September 1980. Claremont Hotel, Berkeley, California, held October 29 - November 9, 1979.

[37] A. A. Garren. Final focusing of the ion beams of a pellet fusion reactor by quadrupole doublets. In Proceedings of the ERDA Summer Study of Heavy Ions for Inertial Fusion, 
pages 102-109, December 1976. Claremont Hotel, Oakland/Berkeley, California, held July 19-30, 1976 .

[38] Herbert Goldstein. Classical Mechanics. Addison-Wesley Publishing Company, second edition, 1980.

[39] I. S. Gradshteyn and I. M. Ryzhik. Table of Integrals, Series, and Products. Academic Press, Inc., corrected and enlarged edition, 1979.

[40] D. P. Grote. Three Dimensional Simulations of Space Charge Dominated Heavy Ion Beams with Applications to Inertial Fusion Energy. PhD thesis, University of California, Davis; Lawrence Livermore National Laboratory, 7000 East Avenue, Livermore, CA 94550-9234, November 1994.

[41] D. P. Grote and A. Friedman. 3D simulations of axially confined heavy ion beams in round and square pipes. Particle Accelerators, 37-38:141-149, 1992.

[42] David P. Grote. WARP Manual. Lawrence Livermore National Laboratory, 7000 East Avenue, Livermore, CA 94550-9234, July 1998.

[43] I. Haber. Numerical simulation of space charge aberrations in final focusing. In Proceedings of the Heavy Ion Fusion Workshop, pages 391-395, September 1980. Claremont Hotel, Berkeley, California, held October 29 - November 9, 1979.

[44] Klaus Halbach. Production of achromatic spots with a beam transport system consisting only of quadrupoles and/or solenoids. In Proceedings of the Heavy Ion Fusion Workshop, page 109, October 1977. Brookhaven National Laboratory, Upton, New York, held October 17-21, 1977. 
[45] William B. Herrmannsfeldt. Final focusing and transmission to target. In ERDA Summer Study of Heavy Ions for Inertial Fusion, pages 25-28, December 1976. Claremont Hotel, Oakland/Berkeley, California, held July 19-30, 1976.

[46] D. D.-M. Ho, K. R. Crandall, and I. Haber. Achromatism in final focusing systems for high-current heavy-ion beams. Particle Accelerators, 37-38:155-160, 1992.

[47] D. D.-M. Ho, K. R. Crandall, and I. Haber. Focusing beams with widely varying current using fixed strength quadrupoles for heavy-ion inertial fusion. Preprint UCRLJC-112144, Lawrence Livermore National Laboratory, 7000 East Avenue, Livermore, CA 94550-9234, October 1992.

[48] D. D.-M. Ho, I. Haber, K. R. Crandall, and S. T. Brandon. Octupole correction of geometric aberrations for high-current heavy-ion beams. Particle Accelerators, 36:141$160,1991$.

[49] R. W. Hockney and J. W. Eastwood. Computer Simulation using Particles. McGrawHill, New York, 1981.

[50] I. Hofmann, R. W. Hasse, and M. Reiser. Intensity effects in final focusing for heavy ion inertial fusion. Technical Report GSI-92-50, Gesellschaft für Schwerionenforschung mbH, Darmstadt, Germany, August 1992.

[51] Jack Hovingh, Victor O. Brady, Andris Faltens, and Denis Keefe. Heavy-ion linear induction accelerators as drivers for inertial fusion power plants. Fusion Technology, 13(2):255-278, February 1988. 
[52] International Atomic Energy Agency. Energy from Inertial Fusion. IAEA, Vienna, Austria, 1995.

[53] International Energy Agency. Energy Technologies for the 21st Century. OECD/IEA, Paris, France, 1997.

[54] John David Jackson. Classical Electrodynamics. John Wiley \& Sons, second edition, 1975.

[55] I. M. Kapchinskij and V. V. Vladimirskij. Limitations of proton beam current in a strong focusing linear accelerator associated with the beam space charge. In International Conference on High-Energy Accelerators and Instrumentation, pages 274-288. CERN, 1959.

[56] Allan N. Kaufman. Lecture notes for Physics 242A: Theoretical Plasma Physics. University of California, Berkeley, 1997.

[57] Allan N. Kaufman. Lecture notes for Physics 205A: Advanced Dynamics. University of California, Berkeley, 1998.

[58] Denis Keefe. Inertial confinement fusion. Annual Review of Nuclear and Particle Science, 32:391-441, 1982.

[59] R. A. Kishek, J. J. Barnard, and D. P. Grote. Effects of quadrupole rotations on the transport of space-charge-dominated beams: Theory and simulations comparing linacs with circular machines. Proceedings of the 1999 Particle Accelerator Conference, New York, pages 1761-1763, 1999. 
[60] Geoffrey Krafft. A heavy ion fusion final focus system. Technical Report LBL-11825, UC-20b, HIFAN-134, Lawrence Berkeley National Laboratory, October 1980.

[61] L. D. Landau and E. M. Lifshitz. Mechanics, volume 1 of Course in Theoretical Physics, chapter $§ 30$. Motion in a rapidly oscillating field., pages 93-95. Pergamon Press, 1960.

[62] L. Jackson Laslett. Selected works of L. Jackson Laslett. Technical Report PUB-616, Lawrence Berkeley National Laboratory, September 1987. Volume II, pages 3-113 3-123.

[63] Lawrence Livermore National Laboratory Lasers Program. Inertial fusion energy: Opportunity for fusion innovation. Technical Report UCRL-MI-125743, Lawrence Livermore National Laboratory, 7000 East Avenue, Livermore, CA 94550-9234, January 1997.

[64] J. D. Lawson. Spherical aberration from non-uniform space-charge. In Proceedings of the Heavy Ion Fusion Workshop, page 396, September 1980. Claremont Hotel, Berkeley, California, held October 29 - November 9, 1979.

[65] E. P. Lee, S. S. Yu, and W. A. Barletta. Phase-space distortion of a heavy-ion beam propagating through a vacuum reactor vessel. Nuclear Fusion, 21(8):961-972, 1981.

[66] Edward P. Lee. Kinetic theory of a relativistic beam. The Physics of Fluids, 19(1):6069, January 1976.

[67] Edward P. Lee. The beam envelope equation — systematic solution for a periodic quadrupole lattice with space charge. Particle Accelerators, 52:115-132, 1996. 
[68] S. MacLaren. A high current density contact ionization source for heavy-ion fusion. Proceedings of the 1999 Particle Accelerator Conference, New York, pages 2849-2851, 1999.

[69] S. MacLaren. Preliminary results from a scaled final focus experiment for heavy ion inertial fusion. Proceedings of the 1999 Particle Accelerator Conference, New York, pages 2852-2854, 1999.

[70] S. A. MacLaren, M. de Hoon, A. Faltens, W. Ghiorso, and P. Seidl. Results from the scaled final focus experiment. In Nuclear Instruments and Methods in Physics Research, Section A, Amsterdam, Netherlands, 2001. Elsevier. 13th International Symposium on Heavy Ion Inertial Fusion, San Diego, California, held March 13-17, 2000.

[71] Stephan Alexander MacLaren. Final focus scaled experiment, Logbook II. Page 177, $1997-2000$.

[72] Stephan Alexander MacLaren. A Scaled Final Focus Experiment for Heavy Ion Fusion. PhD thesis, University of California, Berkeley, 2000.

[73] Philip Francis Meads, Jr. I. The Theory of Aberrations of Quadrupole Focusing Arrays. II. Ion Optical Design of High Quality Extracted Synchrotron Beams with Application to the Bevatron. PhD thesis, University of California, 1963.

[74] Wayne R. Meier. HIBEAM output. Lawrence Livermore National Laboratory, July 1998.

[75] R. W. Moir, M. G. Adamson, R. O. Bangerter, R. L. Bieri, R. H. Condit, C. W. Hartman, P. A. House, A. B. Langdon, B. G. Logan, C. D. Orth, R. W. Petzoldt, 
J. H. Pitts, R. F. Post, R. A. Sacks, M. T. Tobin, W. H. Williams, T. J. Dolan, G. R. Longhurst, M. A. Hoffman, V. E. Schrock, P. F. Peterson, R. Y. Bai, X. M. Chen, J. C. Liu, D.-K. Sze, and W. R. Meier. The HYLIFE-II progress report. Technical Report UCID-21816, Lawrence Livermore National Laboratory, 7000 East Avenue, Livermore, CA 94550-9234, December 1991.

[76] Zane Mottler, Lee Busby, and Fred N. Fritsch. The Python Graphics Interface, Part IV: Python Gist Graphics Manual. Lawrence Livermore National Laboratory, 7000 East Avenue, Livermore, CA 94550-9234, November 1998. UCRL-MA-128569, Manual 4.

[77] David Neuffer. Geometric aberrations in final focussing for heavy ion fusion. Proceedings of the Heavy Ion Fusion Workshop, pages 333-339, September 1978. Argonne National Laboratory, Argonne, Illinois, held September 19-26, 1978.

[78] Dwight R. Nicholson. Introduction to Plasma Theory. Krieger Publishing Company, Malabar, Florida, 1992.

[79] Katsunobu Oide. Design of optics for the final focus test beam at SLAC. Technical Report SLAC-PUB-4953, Stanford Linear Accelerator Center, May 1989. Presented at the IEEE Particle Accelerator Conference, Chicago, Illinois, March 20-23, 1989.

[80] William H. Press, Saul A. Teukolsky, William T. Vetterling, and Brian P. Flannery. Numerical Recipes in C; The Art of Scientific Computing. Cambridge University Press, second edition, 1992.

[81] Lionel Prost. Final focus scaled experiment, Logbook II. Pages 179-192, 1997-2000. 
[82] M. Reiser. Physics of intense charged-particle beams for heavy-ion inertial fusion. Fusion Engineering and Design, 32-33:133-140, 1996.

[83] Martin Reiser. Theory and Design of Charged Particle Beams. Wiley Series in Beam Physics and Accelerator Technology. John Wiley \& Sons, Inc., 1994.

[84] Robert D. Richtmyer and K. W. Morton. Difference Methods for Initial-Value Problems, chapter 12. John Wiley \& Sons, Inc., second edition, 1967.

[85] J. M. Roberts. The Penguin History of the World. Penguin Books, London, England, third edition, 1995.

[86] Mordecai David Rosen. Lecture notes for Nuclear Engineering 180: Fusion Technology. University of California, Berkeley, 1996.

[87] P. A. Seidl, F. M. Bienosek, C. M. Celata, A. Faltens, J. W. Kwan, S. A. MacLaren, D. Ponce, D. Shuman, S. Yu, L. Ahle, S. Lund, A. Molvik, and C. M. Sangster. Experiments at the Virtual National Laboratory for Heavy Ion Fusion. In Nuclear Instruments and Methods in Physics Research, Section A, Amsterdam, Netherlands, 2001. Elsevier. 13th International Symposium on Heavy Ion Inertial Fusion, San Diego, California, held March 13-17, 2000.

[88] W. M. Sharp, A. Friedman, and D. P. Grote. Effects of longitudinal space charge in beams for heavy-ion fusion. Fusion Engineering and Design, 32-33:201-208, 1996.

[89] W. M. Sharp, D. P. Grote, S. M. Lund, and S. S. Yu. Envelope model of beam transport in ILSE. In Computational Accelerator Physics, Los Alamos, 1993. 
[90] Frank H. Shu. The Physics of Astrophysics, Volume II: Gas Dynamics, chapter 15. University Science Books, Sausalito, California, 1992.

[91] Lloyd Smith. Stability of the K-V distribution in long periodic transport systems. Technical Report HIFAN-13, Lawrence Berkeley National Laboratory, October 1977.

[92] J. Steinhoff. Quadrupole systems for focussing ion beams with large momentum spread. In Proceedings of the Heavy Ion Fusion Workshop, pages 345-354, September 1978. Argonne National Laboratory, Argonne, Illinois, held September 19-26, 1978.

[93] M. Tabak, D. Callahan-Miller, D.D.-M. Ho, and G. B. Zimmerman. Design of a distributed radiation target for intertial fusion driven from two sides with heavy ion beams. Nuclear Fusion, 38(4):509-514, April 1998.

[94] Michael G. Tiefenback. Space-Charge Limits on the Transport of Ion Beams in a Long Alternating Gradient System. PhD thesis, University of California, Berkeley, November 1986.

[95] Guido van Rossum. Python 1.5.2. www.python.org, June 1999. Copyright 1991-1995 Stichting Mathematisch Centrum, Amsterdam.

[96] Guillermo Velarde, Yigal Ronen, and José M. Martínez-Val, editors. Nuclear Fusion by Inertial Confinement: A Comprehensive Treatise. CRC Press, 1993.

[97] T. P. Wangler, K. R. Crandall, R. S. Mills, and M. Reiser. Relation between field energy and RMS emittance in intense particle beams. IEEE Transactions on Nuclear Science, 32(5):2196-2200, October 1985. 
[98] G. N. Watson. A Treatise on the Theory of Bessel Functions, chapter 15.51. Rayleigh's extension of Euler's formula, pages 502-503. Cambridge University Press, second edition, 1980.

[99] World Health Organization. The World Health Report 1998: Life in the 21st Century - A Vision for All. WHO, Geneva, Switzerland, May 1998. 


\section{Appendix A}

\section{Python Scripts Used}

\section{A.1 Drift Compression Design}

The Python script below was used to set up a drift compression section using Hermes, as described in chapter 4 . One backward run is done to set up the lattice, followed by one forward run to assess the effects of rematching or initial errors. The file design990113 contains information about the standard IRE design, as described in table 1.2. The routine sethermesbeam initializes a beam for Hermes. The variable top. straight defines the fraction of the beam in the flat-top part of the beam, whereas her.iprofile specifies the shape of the current falloff at the beam ends. For her.iprofile $=2$, a parabolic falloff is used. If the boolean variable lfinalrematch is set to true, the beam is rematched once the backward run reaches the beginning of the drift compression. Without rematching at the beginning of drift compression, the forward and backward run are identical. The variable stepsize specifies the step size to be used, in units of meters. The function getscaling defines what the average beam radius should be as a function of position along the drift 
compression. At the end of the run, the RMS deviation is calculated between the current at the end of the drift compression and the originally specified current. 


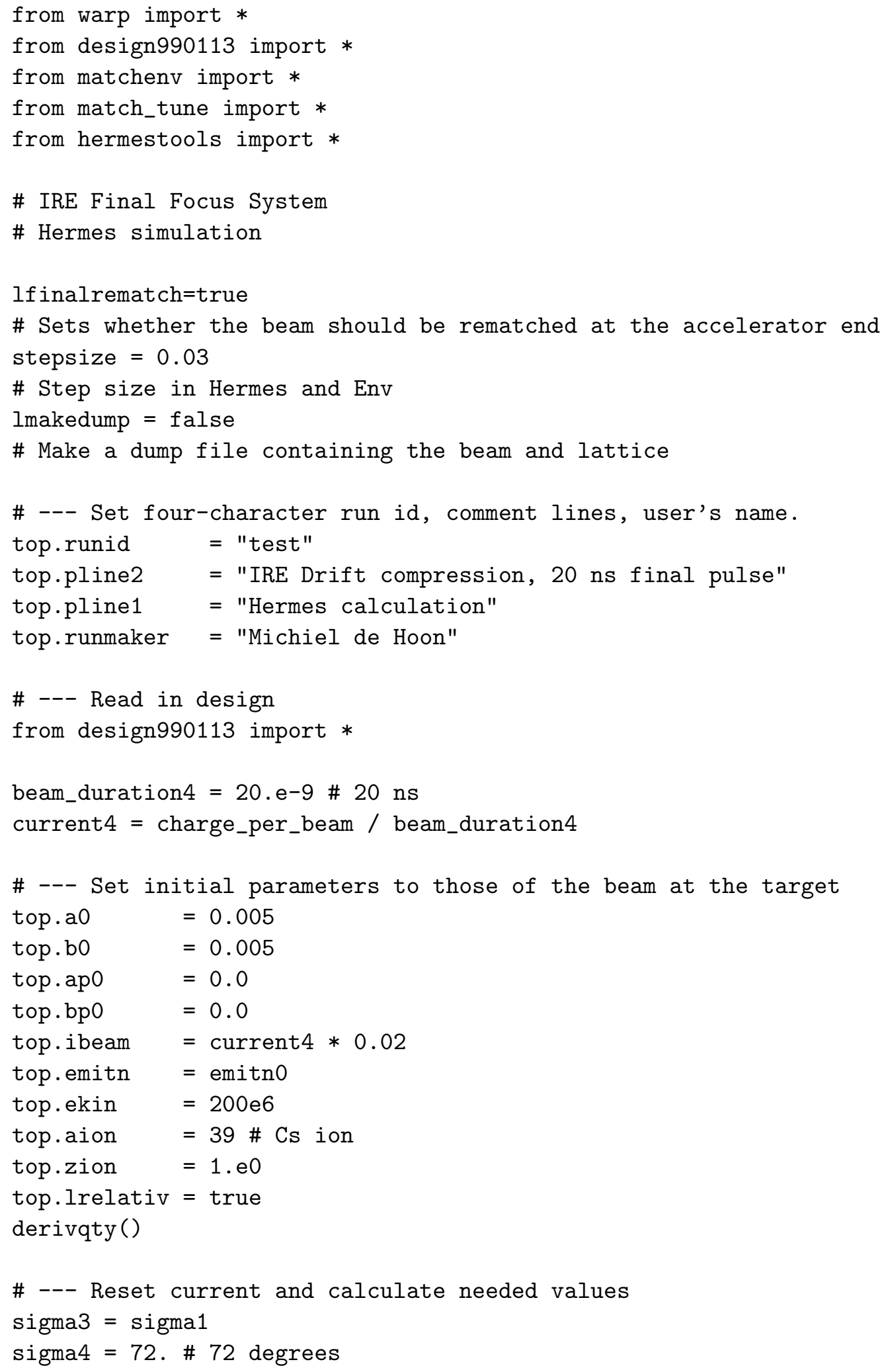




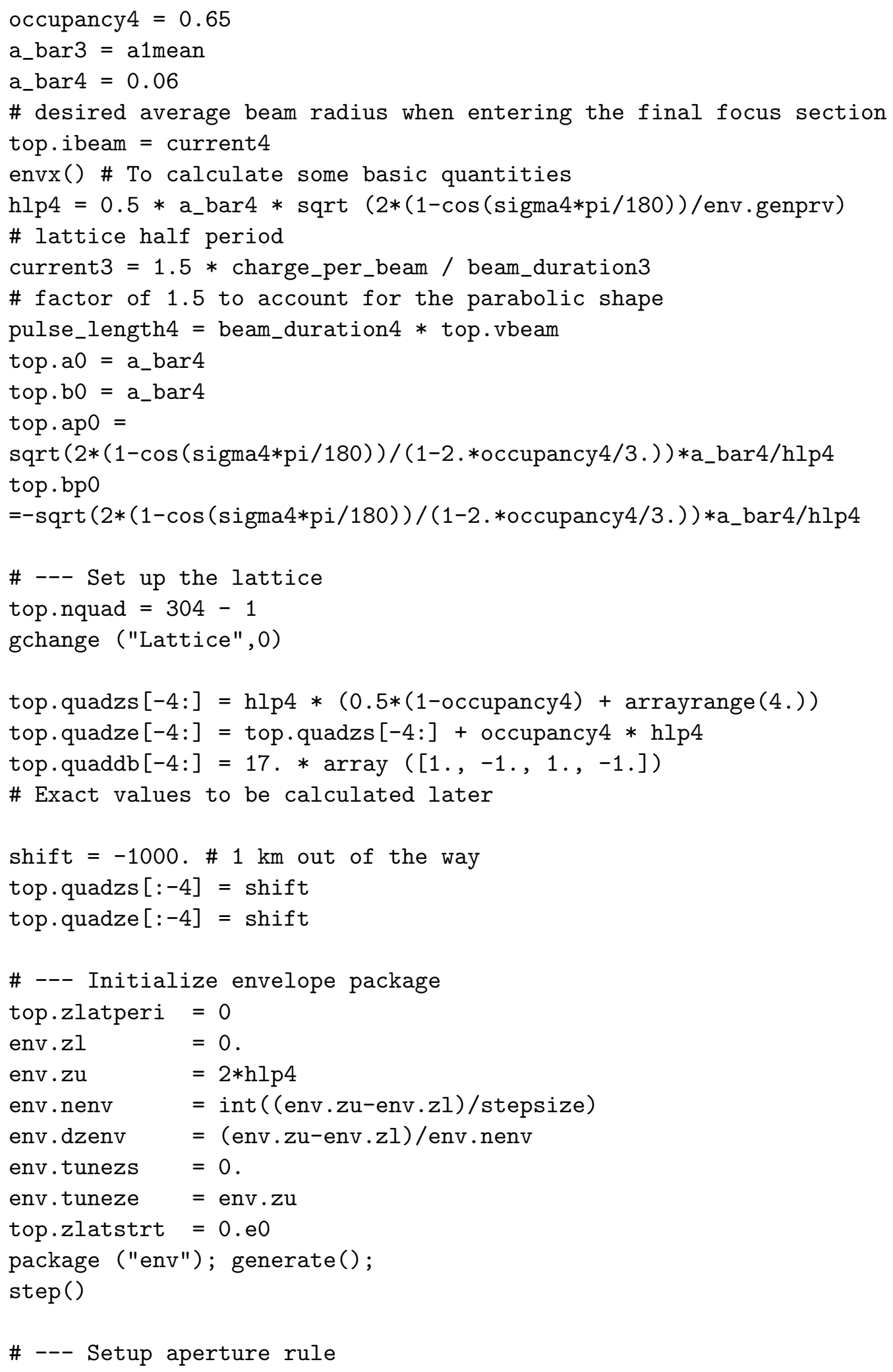




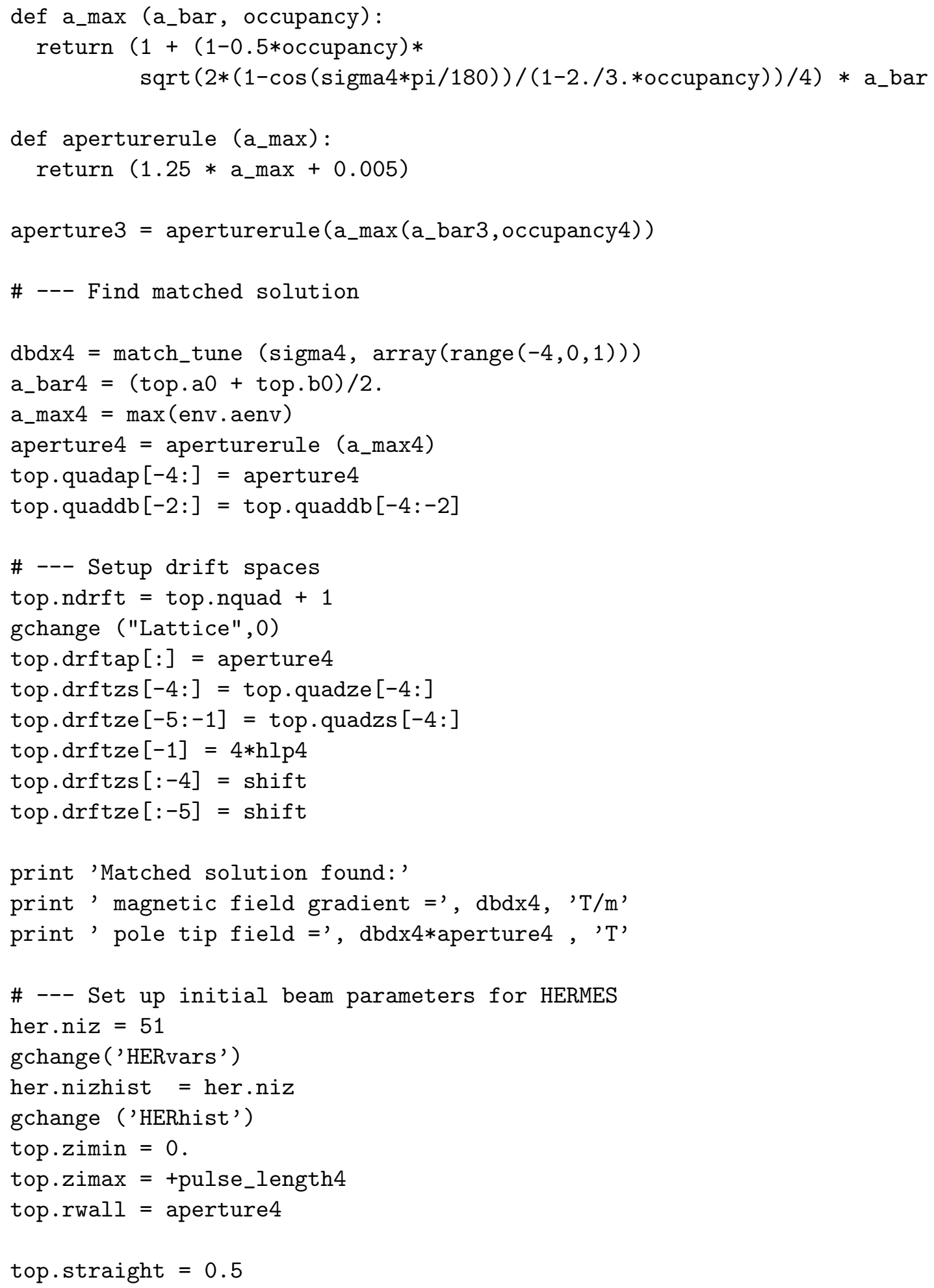




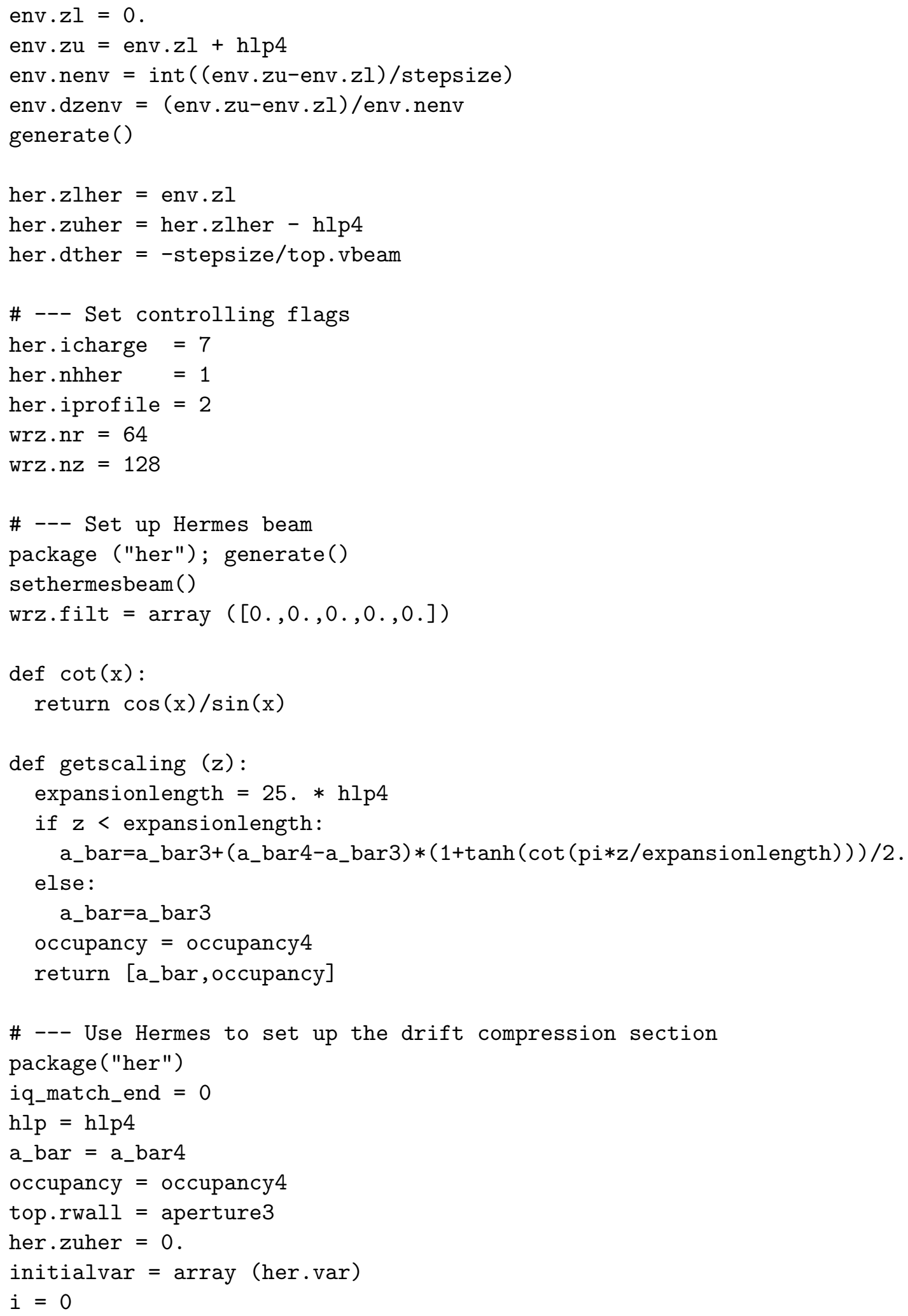




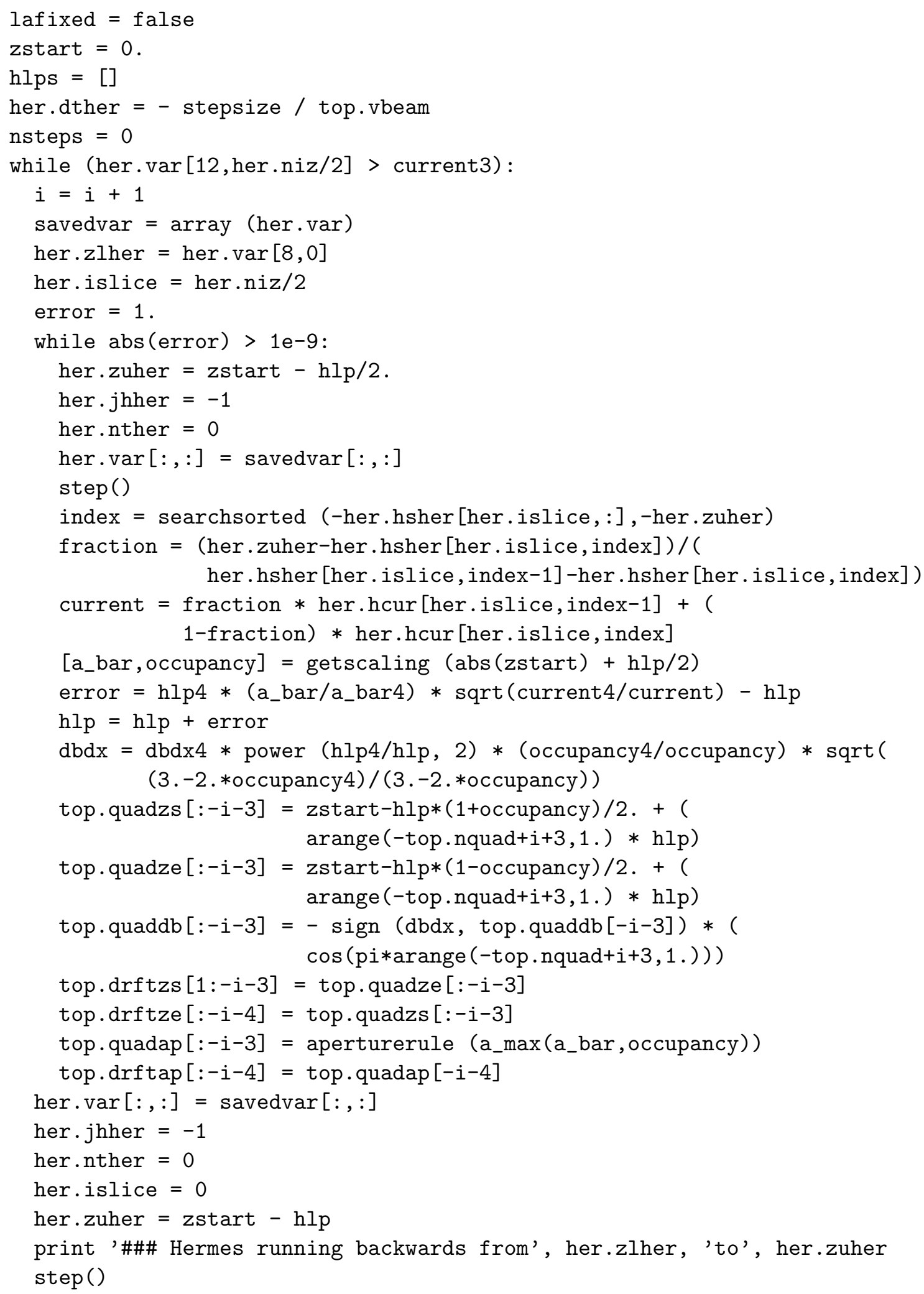




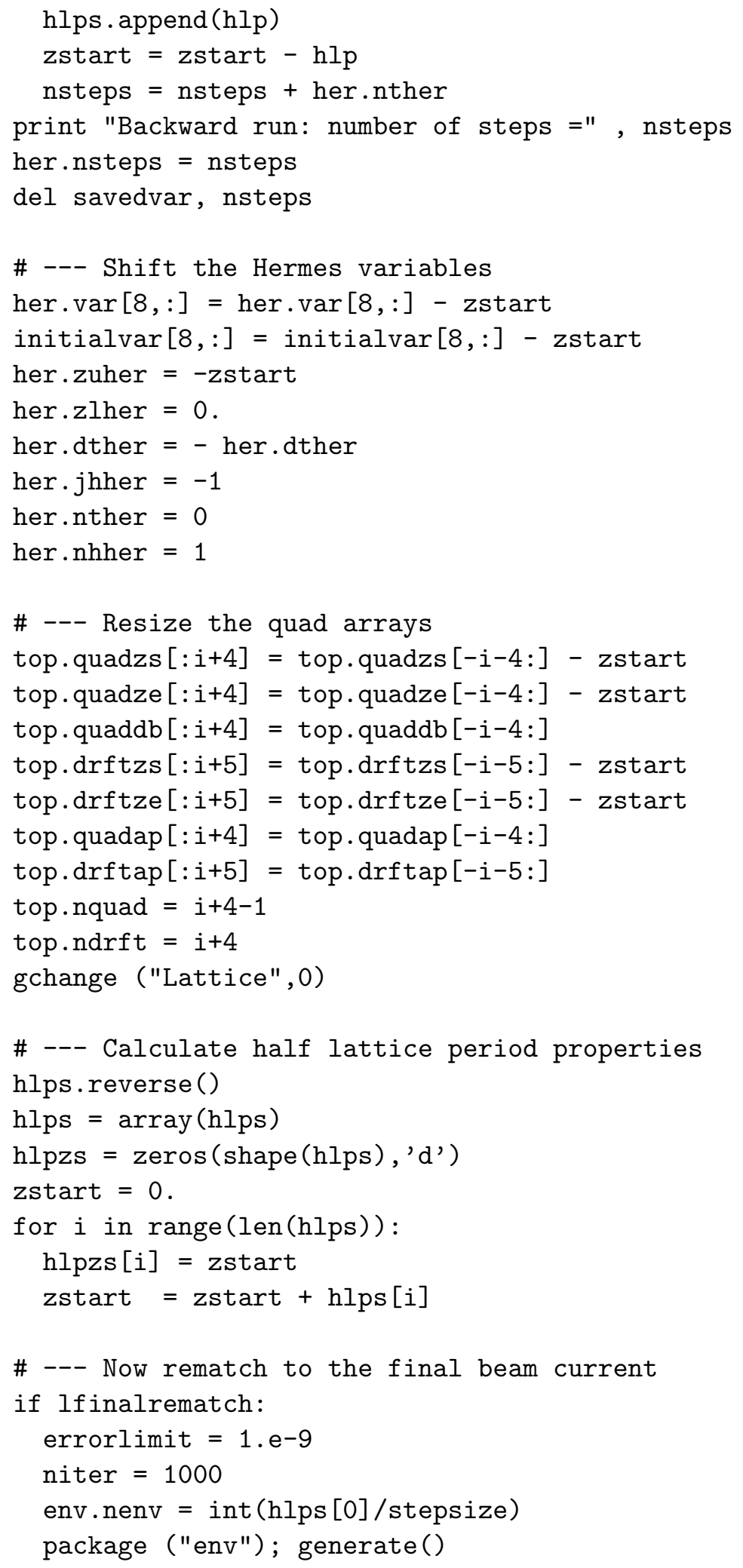




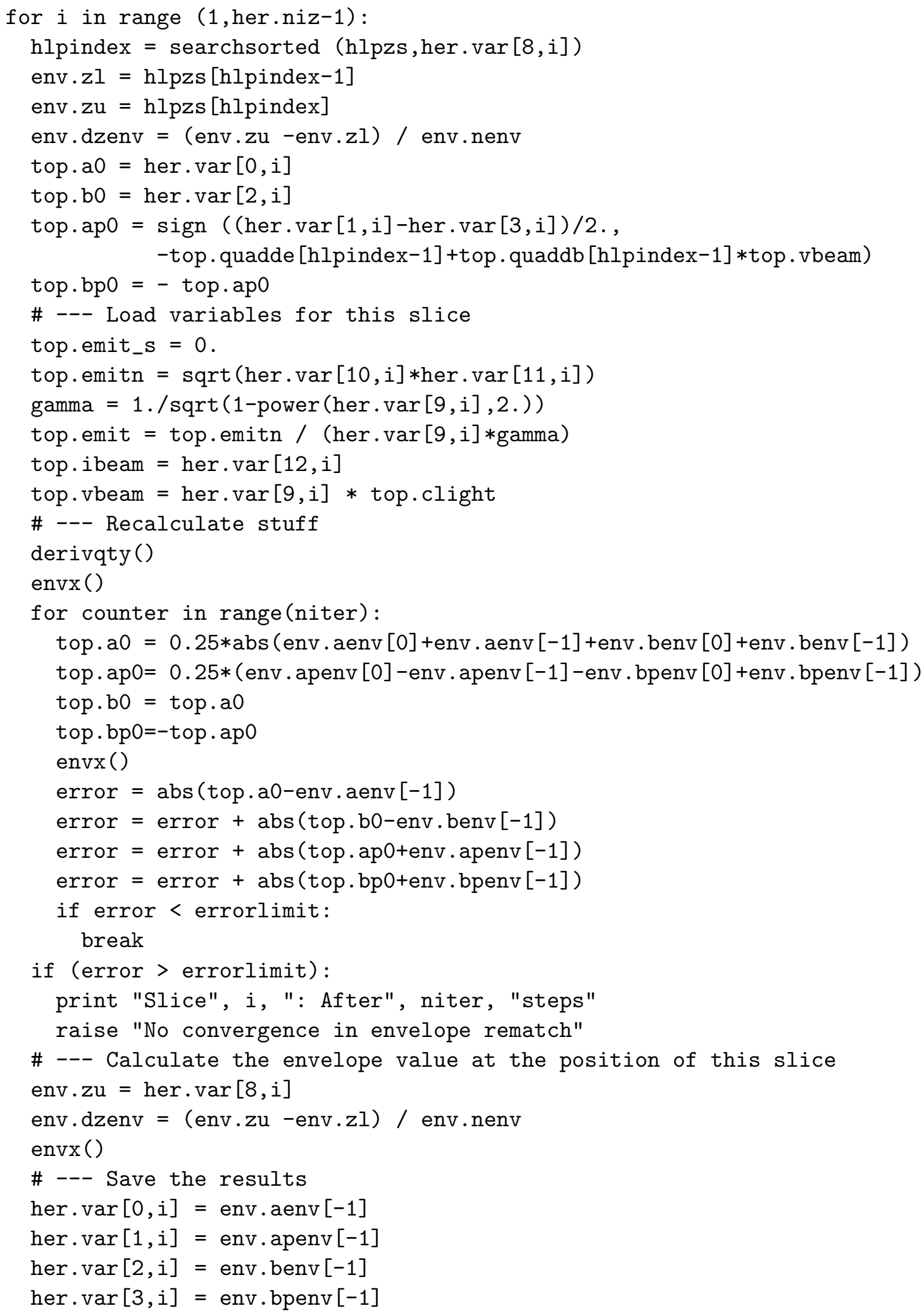




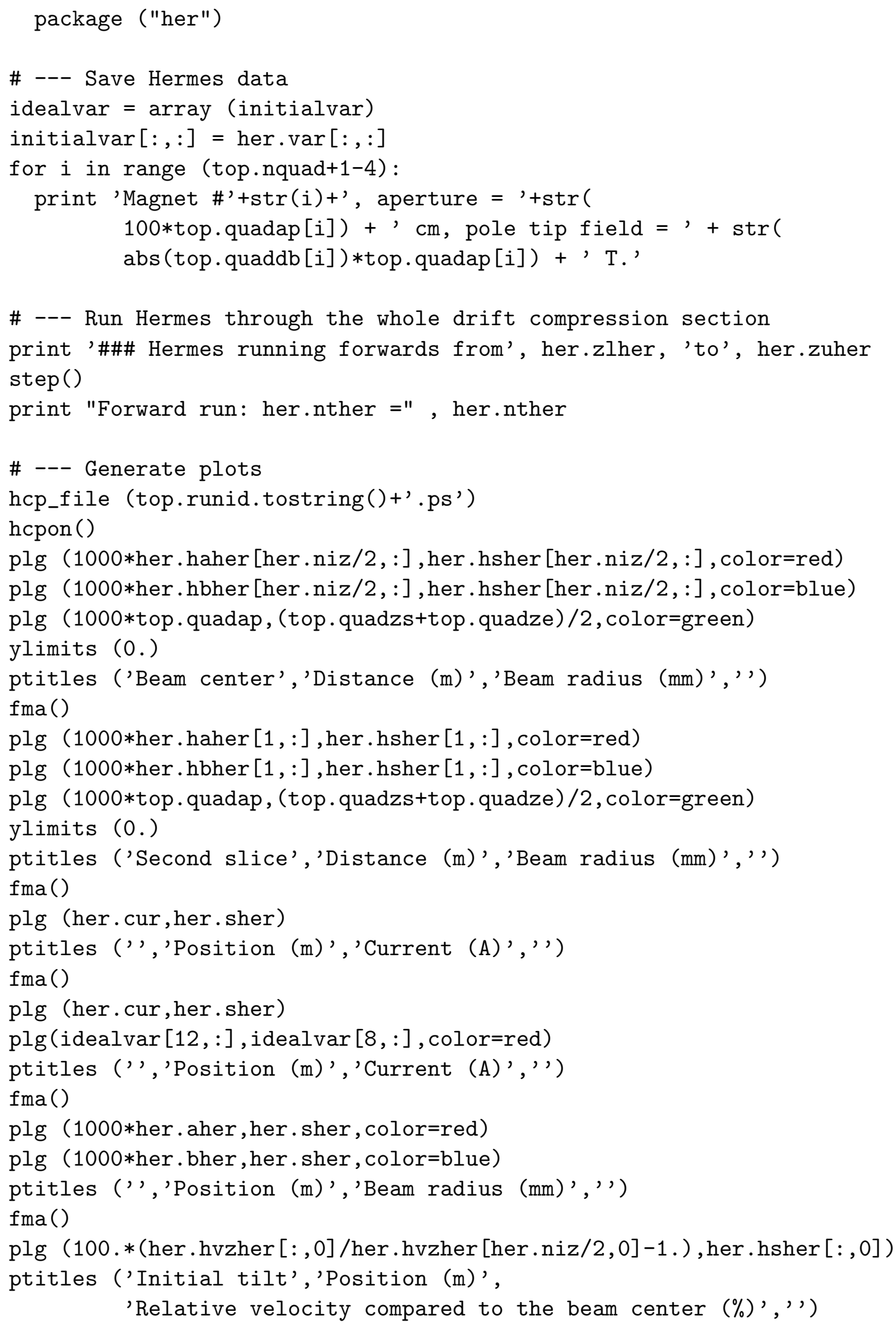




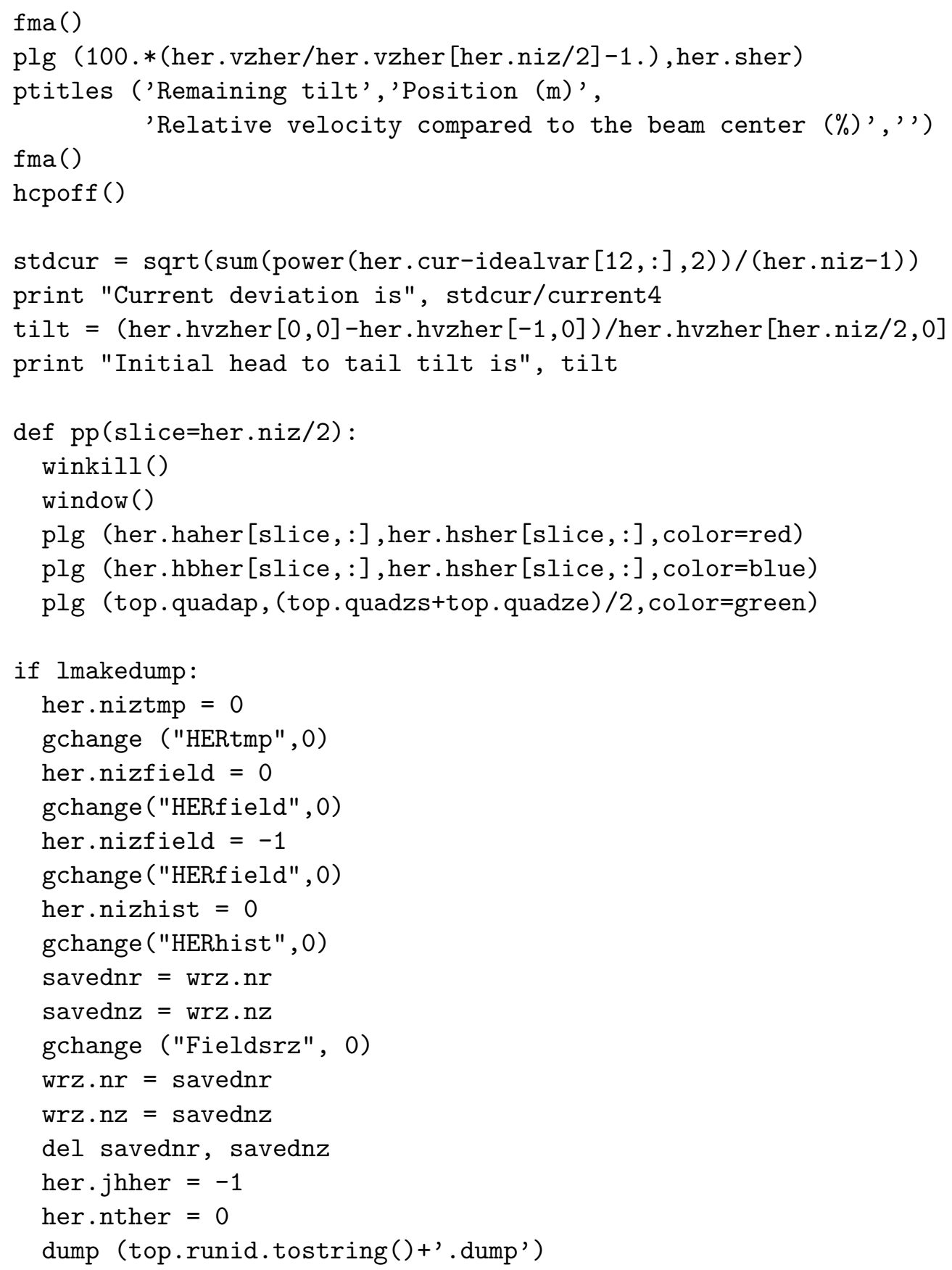




\section{A.2 PIC Code Rematching of Final Focus Systems}

The Python script below was used for rematching a final focus system using the PIC code WARPxy, as described in chapter 5. In the example below, a saved file is loaded in. That line (pyrestore (top.runid.tostring()+'.dump')) can be skipped in order to start a matching iteration from scratch. An iteration sequence is carried out by calling FirstStep() first to find the initial error. Iteration steps can then be performed by calling NextStep(). The convergence angle at the neutralization point that we are looking for is defined by the variable theta. The variable nparticles is the number of particles to be used for the WARPxy runs, while averagequadstep and averagestep are the step sizes to be used inside and outside of the quadrupoles and their fringe fields. If 1samestep is set to true, the step size will be equal to averagestep throughout. If any of these control variables or theta is changed, FirstStep() should be called first before making iteration steps by calling NextStep(). The convergence angle found in the simulation is compared to the desired convergence angle, and the deviation of $a^{\prime}$ and $b^{\prime}$ from zero and $a$ and $b$ from each other is calculated on each iteration step. The code returns the total error in these quantities every time FirstStep() or NextStep() is called. This script is based on the Newton-Raphson method for nonlinear systems of equations [80]. 
from warp import *

from fringedquads import *

from LinearAlgebra import *

\# IRE Final Focus System

\# 2D slice simulation

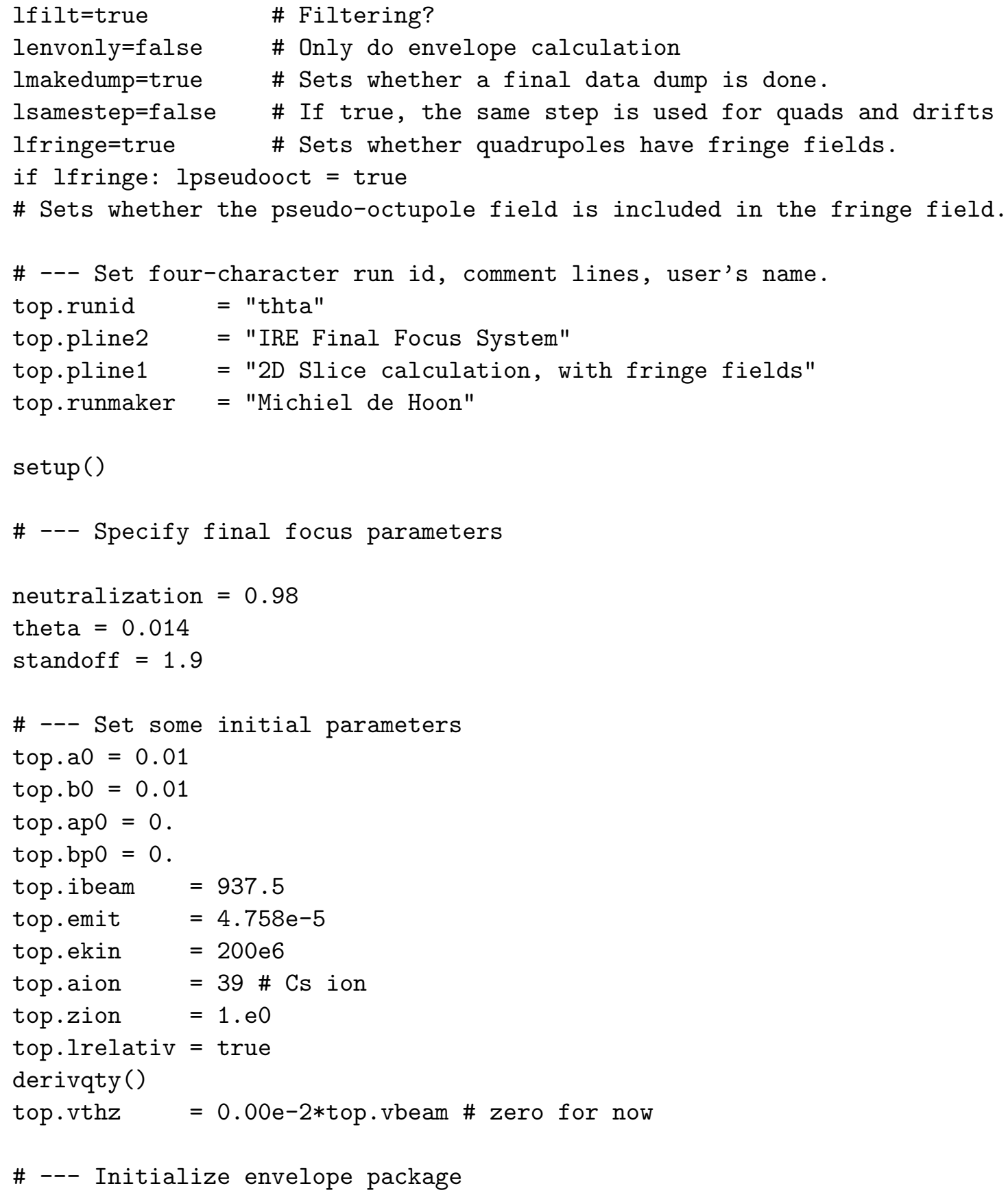




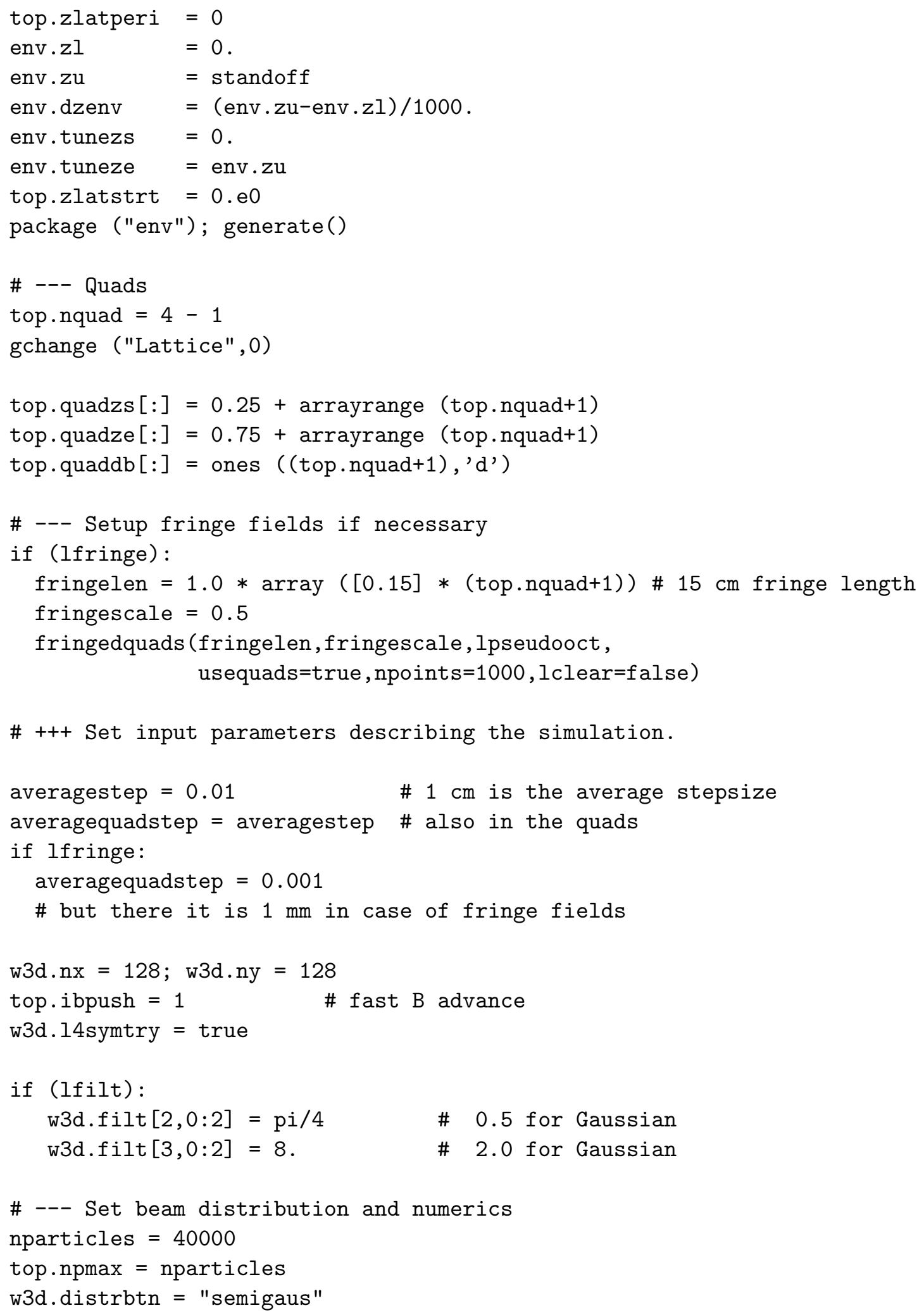




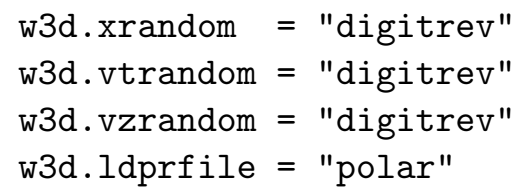




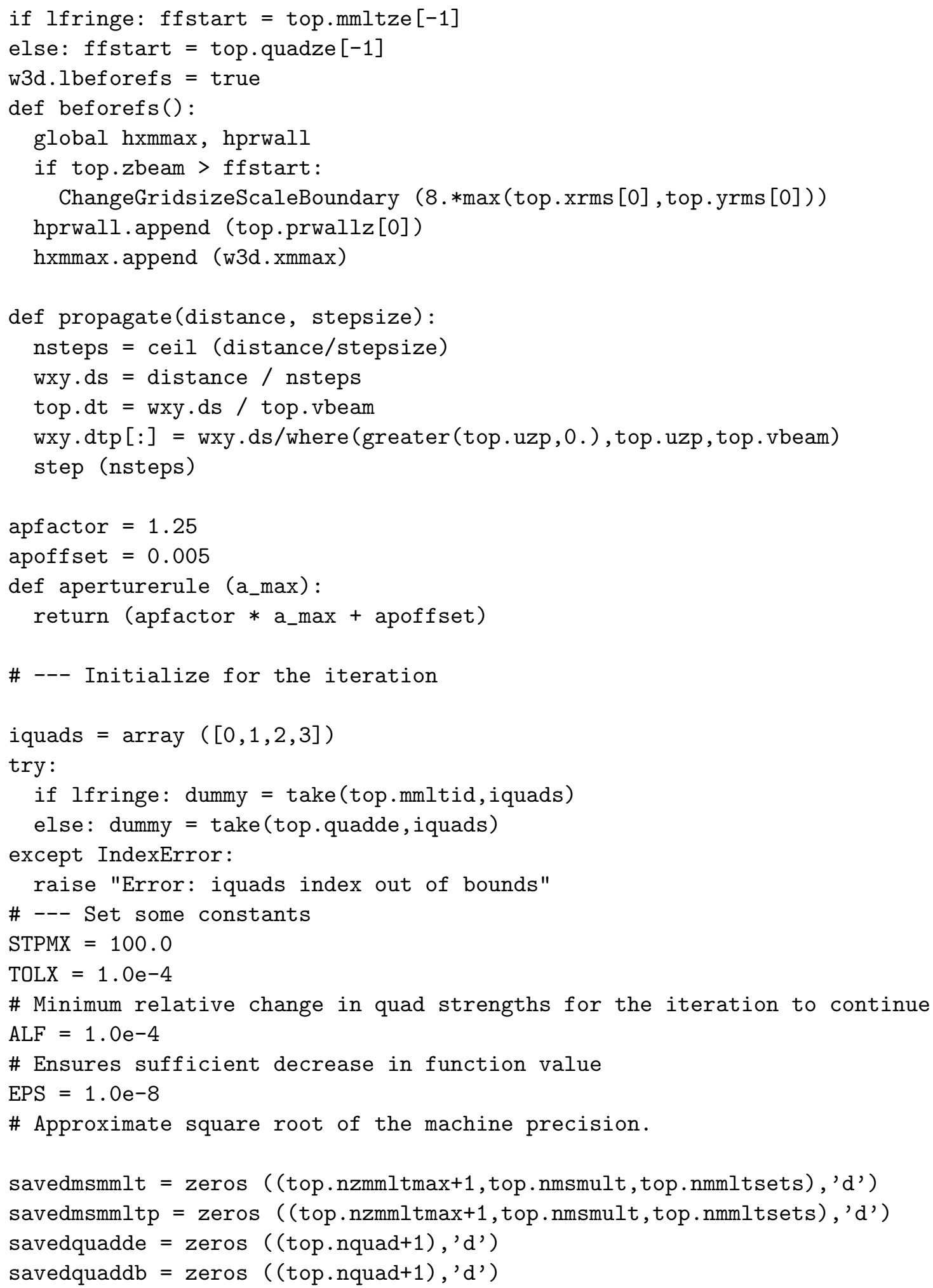


\# --- Read in saved data

pyrestore (top.runid.tostring()+'.dump')

from fixlogicals import *

fixlogicals()

\# --- Define functions needed for iteration

def Initialize():

global hprwall, hxmmax

\# --- Re-initialize the slice package

top.lprntpara $=$ false

top. verbosity $=0$

top.zbeam $=0$.

top.zgrid $=0$.

top.zgridprv $=0$.

top.it $=0$.

top.time $=0$.

top. jhist $=-1$

top.np_s $=$ array ([0], 'i')

top.npmax = nparticles

\# --- Set grid size in the starting channel

top.prwall $=$ aperturerule $(\max (\max (e n v \cdot \operatorname{aenv}), \max (e n v \cdot b e n v)))$

w3d.xmmax $=$ top.prwall $/ 0.95$

$\mathrm{w} 3 \mathrm{~d} \cdot \mathrm{ymmax}=\mathrm{w} 3 \mathrm{~d} \cdot \mathrm{xmmax}$

$\mathrm{w} 3 \mathrm{~d} \cdot \mathrm{xmmin}=-\mathrm{w} 3 \mathrm{~d} \cdot \mathrm{xmmax}$

$\mathrm{w} 3 \mathrm{~d} \cdot \mathrm{ymmin}=-\mathrm{w} 3 \mathrm{~d} \cdot \mathrm{ymmax}$

\# --- Initialize history arrays

hprwall $=[$ top.prwall $]$

$\mathrm{hxmmax}=[\mathrm{w} 3 \mathrm{~d} \cdot \mathrm{xmmax}]$

\# --- Initialize for the generate

distance $=$ top.quadzs [0]

nsteps $=$ ceil (distance $/$ averagestep)

wxy.ds = distance / nsteps

top.dt $=$ wxy.ds / top.vbeam

\# --- Generate the PIC code

$\mathrm{w} 3 \mathrm{~d} \cdot \mathrm{nz}=2$

$\mathrm{w} 3 \mathrm{~d} \cdot \mathrm{zmmin}=0$.

w3d.zmmax $=0$. \# Needed for parallel running

\# --- Set up the boundary condition

top.fstype $=1$

symm_fact $=1$.

if (w3d.12symtry): symm_fact $=0.5$

if (w3d.14symtry): symm_fact $=0.25$ 


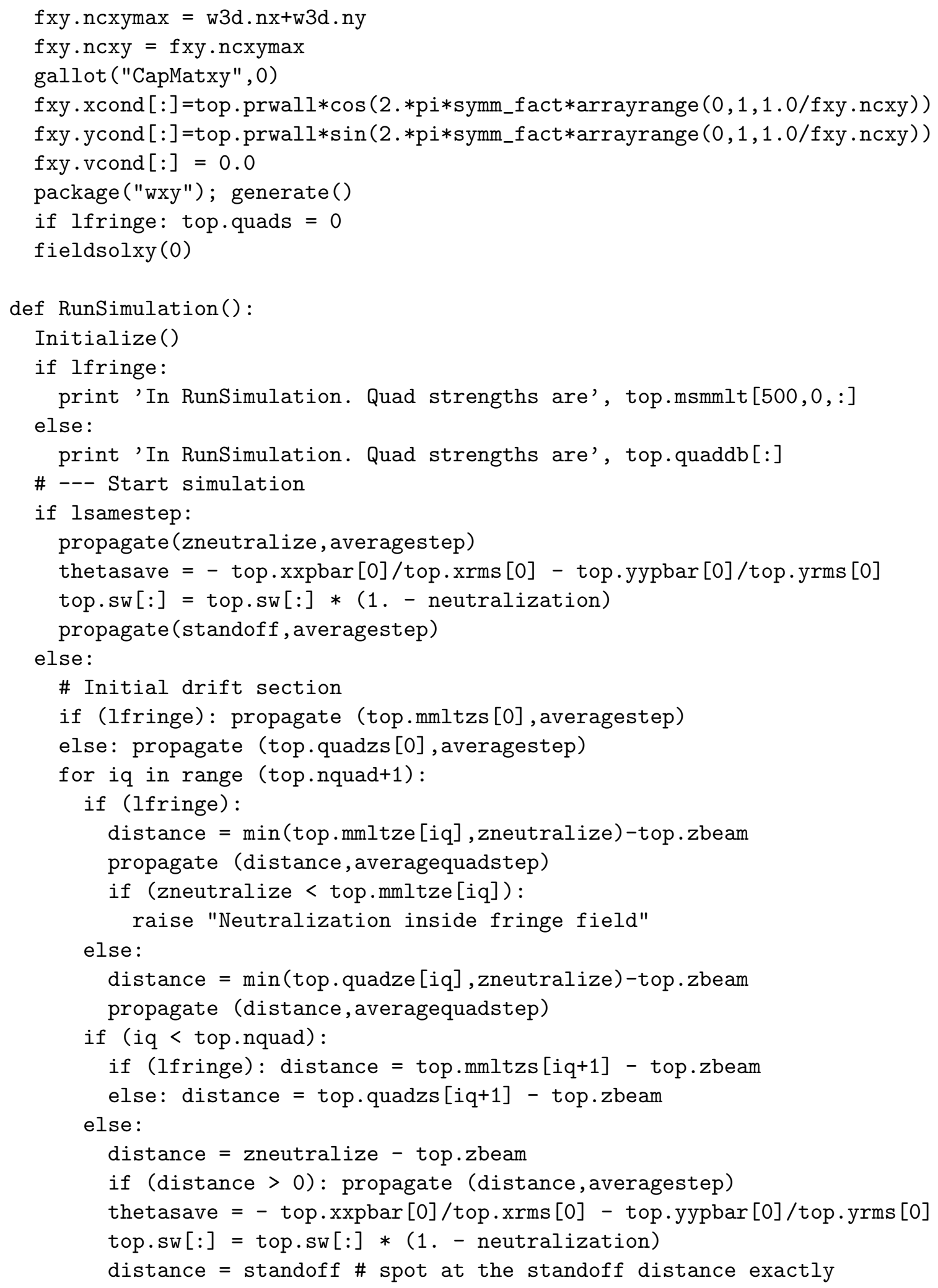




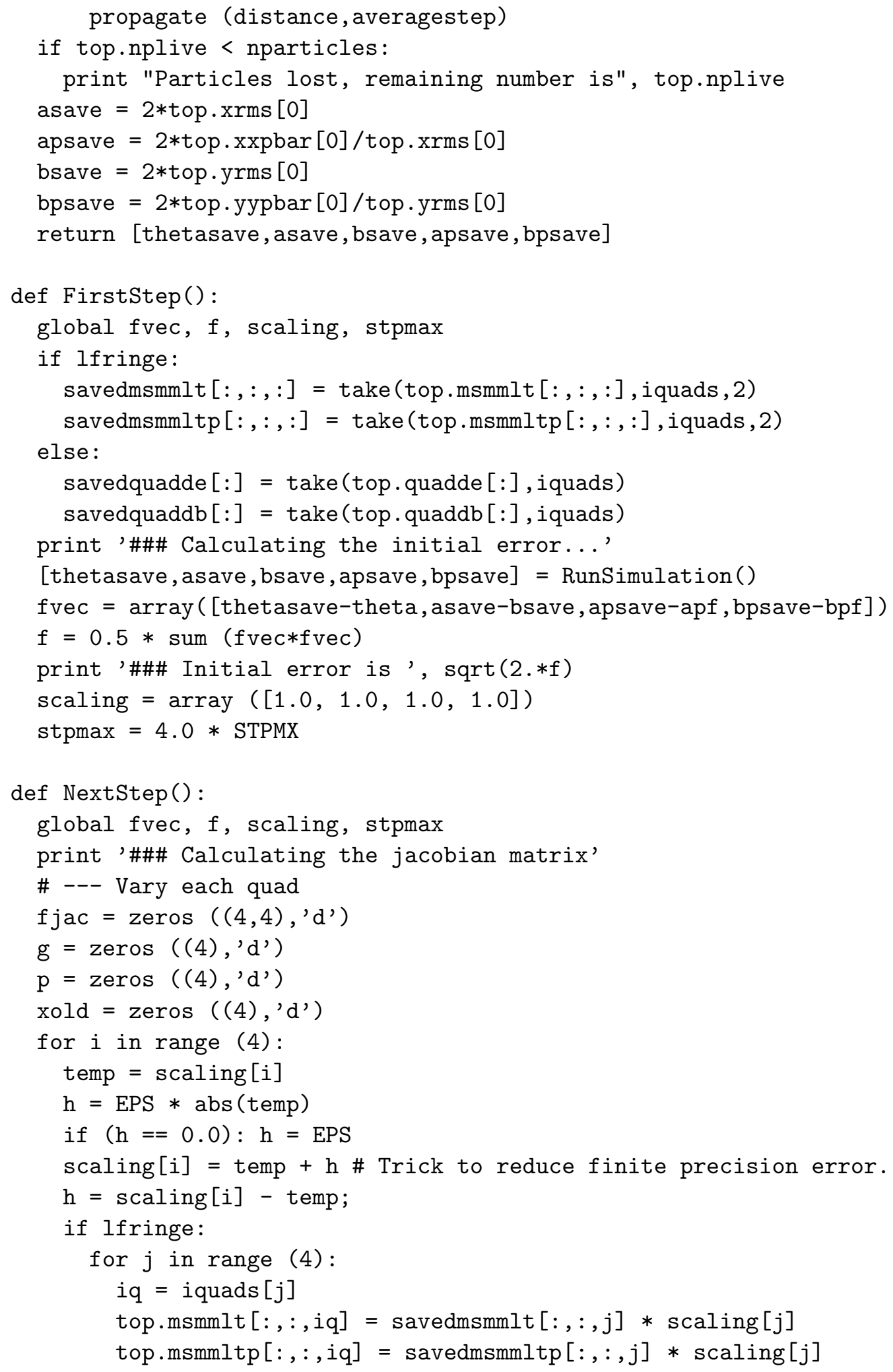




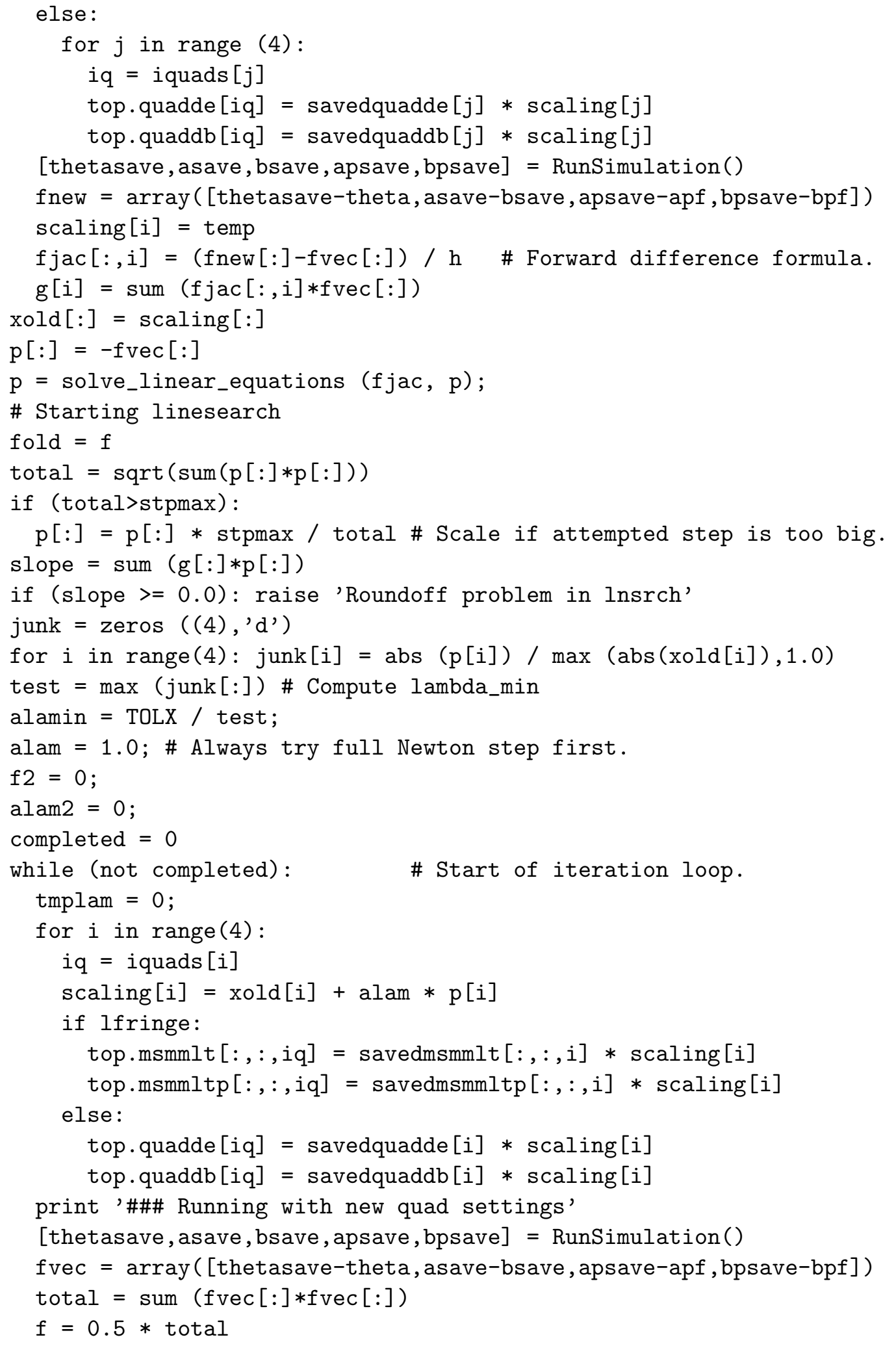




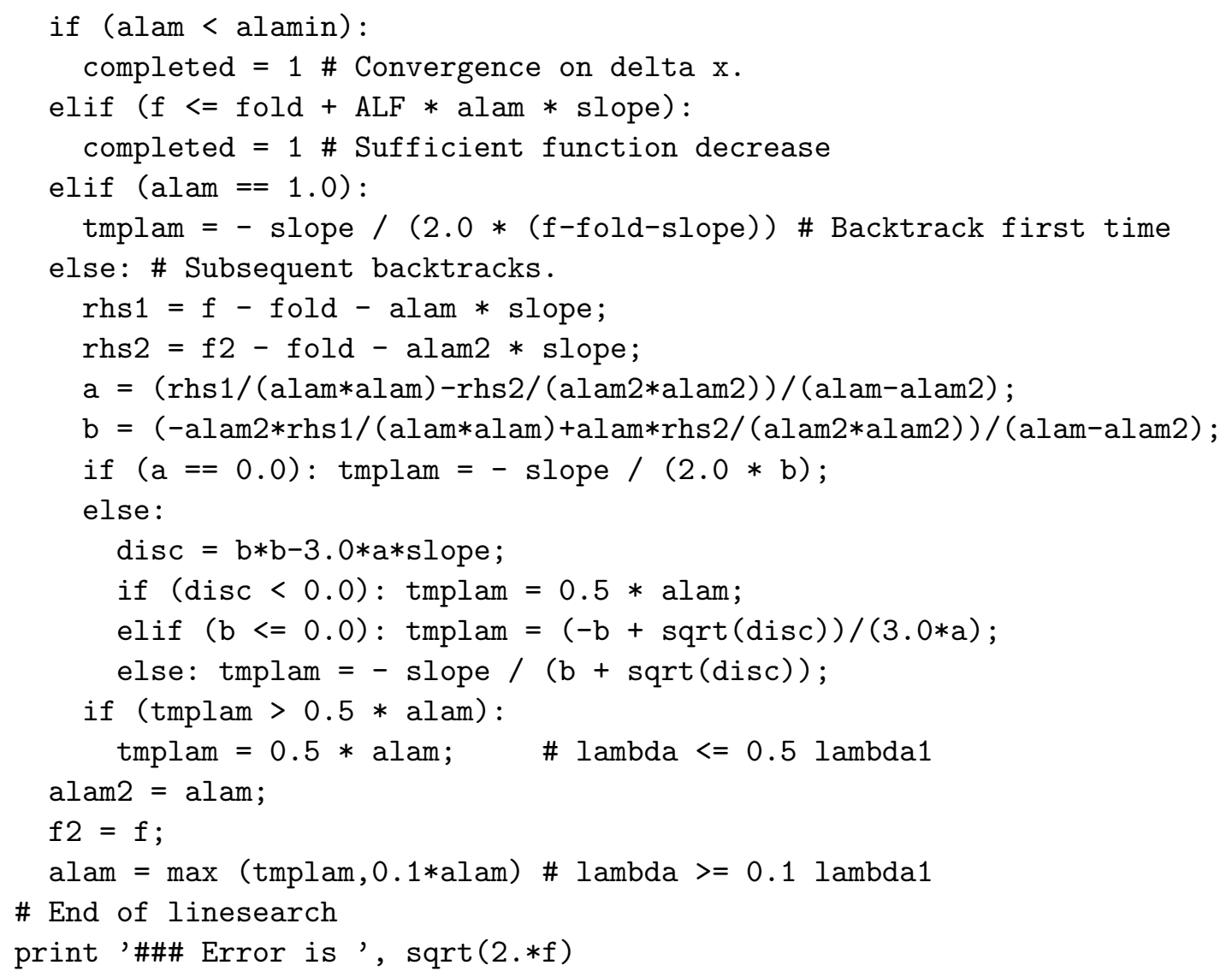

